\title{
Estudio de Líneas de Helio en \\ Estrellas Peculiares Tempranas
}

Autor: Lic. Rodolfo Enrique Vallverdú

Directora: Dra. Lydia Sonia Cidale

Tesis Doctoral en Astronomía

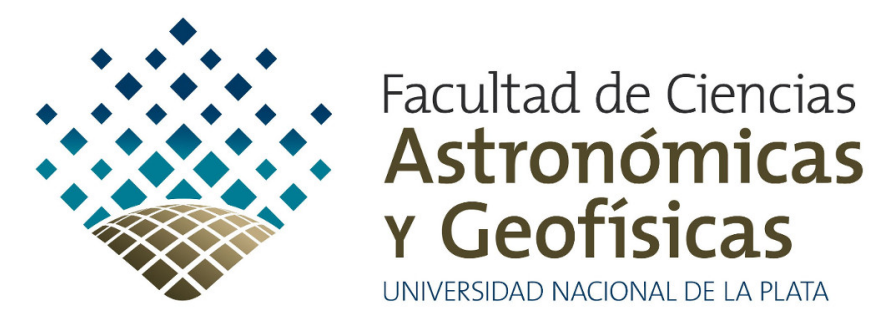

31 de octubre de 2016 
A todos mis afectos y a aquellos que de alguna forma han contribuido a este trabajo. 


\section{Resumen}

Las estrellas de tipo espectral A y B químicamente peculiares son rotadores lentos y presentan intensificaciones o deficiencias de algunas especies químicas en sus atmósferas, en comparación con las características que presentan las estrellas enanas A y B normales de la misma temperatura efectiva Jaschek y Jaschek 1987. Se piensa que las anomalías químicas están causadas por fenómenos que han tenido lugar después de la formación de la estrella, tales como la difusión y los campos magnéticos Michaud 1970.

Actualmente se conoce una importante fracción de estrellas masivas, aproximadamente un 10\%, que presentan intensos campos magnéticos de gran escala Grunhut et al. 2012, Wade y MiMeS Collaboration, 2015. Además, es sabido que el campo magnético influye significativamente en los procesos físicos que operan en el interior (rotación interna, procesos de mezcla, difusión, etc.) y entorno de las estrellas (confinamiento magnético, pérdida de masa, anomalías químicas en la superficie), con consecuencias en la estructura y evolución estelar. Sin embargo, estos temas han sido muy poco estudiados entre las estrellas de la secuencia principal.

Un grupo de estrellas de particular interés para estudiar la influencia de los campos magnéticos lo constituyen las estrellas peculiares en helio (He). Estas estrellas conocidas como He-weak (deficientes en He) y He-strong (enriquecidas en He) suelen presentar variaciones fotométricas y espectroscópicas que correlacionan con la rotación de la estrella. Muchas de ellas presentan intensos campos magnéticos (del orden de algunos $\mathrm{kG}$ ) cuya componente longitudinal varía periódicamente. Esta variación es comúnmente interpretada en términos del Modelo de Rotador Oblicuo donde la geometría del campo magnético (supuesta dipolar) no es simétrica respecto al eje de rotación de la estrella. De esta forma, un observador externo ve una configuración magnética que cambia a medida que la estrella rota (Bohlender, 1994).

La mayoría de los modelos actuales que describen la estructura interior, la atmósfera y la evolución de las estrellas desprecian los efectos magnéticos. Muy pocos trabajos han explorado los efectos de la presión magnética en la estructura de la fotosfera de las estrellas peculiares, y todos ellos se han enfocado principalmente al estudio de las estrellas A peculiares Carpenter, 1985, LeBlanc et al. 1994, Valyavin et al. 2004, Shulyak et al. 2007. El modelado de las distribuciones anómalas de elementos químicos (manchas) se realiza 
actualmente proponiendo perfiles de abundancias químicas empíricos Krtička et al. 2013 . El objetivo de este trabajo es estudiar la influencia de un campo magnético sobre la estructura de la atmósfera y su impacto sobre los perfiles de las líneas de He en estrellas peculiares en He. Para ello se modeló la fotosfera, en la aproximación de equilibrio hidrostático, de estrellas B0 a B9 (prototipos de estrellas que presentan anomalías en He) donde se incluyó un término que contiene la fuerza de Lorentz. A tal fin se adaptó el código de atmósferas estelares realizado por Rohrmann 2001); Rohrmann et al. 2002 que resuelve una atmósfera en equilibrio termodinámico local, usando la aproximación de capas plano-paralelas en ausencia de un campo magnético. La incorporación del término de la fuerza de Lorentz se realizó siguiendo el procedimiento de Valyavin et al. 2004 A lo largo del trabajo se analizó el efecto de la componente radial de la fuerza de Lorentz sobre la marcha de la gravedad superficial con la profundidad óptica y la latitud magnética. En todos los casos se consideró un campo dipolar con intensidades entre $0 \mathrm{G}$ y $50000 \mathrm{G}$ (unos 18 modelos) y la fuerza de Lorentz dirigida hacia afuera o hacia adentro de la estrella.

Se discutió, además, la formación de líneas de He en presencia de campos magnéticos. A tal fin se resolvió rigurosamente la ecuación de transporte radiativo, simultáneamente con la ecuación de equilibrio hidrostático. Se encontró que los efectos de la presión magnética son más importantes cuando la fuerza de Lorentz está dirigida hacia afuera. Los cambios de densidad y presión que resultan justifican una variación local en los perfiles de las líneas de He de hasta un 7\%, considerando una inversión del sentido de la fuerza de Lorentz. Luego, sobre la base del modelo de rotador oblicuo se discutió el efecto de un campo magnético medio a nivel global, calculando la contribución integrada al espectro de línea sobre el hemisferio visible de la estrella. Se encuentra que los efectos magnéticos se diluyen en este caso.

Por último se analizó el efecto del campo magnético sobre la difusión. Se resolvieron las ecuaciones de difusión atómica en ausencia y presencia de un campo magnético en una atmósfera estelar, y se determinó el perfil químico resultante con la latitud magnética. En este último caso se discutieron los efectos locales. Encontramos que la difusión en presencia de un campo magnético contribuye a explicar variaciones locales en las líneas de He, de hasta un $10 \%$ en la intensidad y un $25 \%$ en el ancho equivalente con respecto a un modelo sin campo magnético. Además, fue posible identificar regiones con diferencias en la abundancia del He. Cabe destacar que en esta etapa hemos despreciado los efectos de la difusión radiativa. Finalmente, comparamos cualitativamente los resultados teóricos con algunas observaciones de estrellas peculiares en He.

Consideramos que la relevancia de este trabajo reside en que es la primera vez que se realiza un análisis completo, riguroso y consistente del efecto de un campo magnético en la estructura de las fotosferas de las estrellas B de secuencia principal, que incluyen además los mecanismos 
de difusión atómica.

Destacamos la importancia de este trabajo en el modelado de la estructura de una atmósfera y consideramos que es un importante avance para el tratamiento a futuro de un modelo más completo donde se tenga en cuenta el efecto selectivo de la fuerza de radiación, y el cambio en la abundancia química de la estrella como consecuencia de la evolución estelar. 


\section{Índice General}

Resumen I

$\begin{array}{ll}\text { Índice General } & \text { IV }\end{array}$

\begin{tabular}{lll}
\hline 1 & Introducción & 1
\end{tabular}

\begin{tabular}{|lll}
2 & Estrellas químicamente peculiares & 6
\end{tabular}

2.1 Estrellas A y B químicamente peculiares . . . . . . . . . . . . . . . . . 6

2.1 .1 Estrellas A metálicas, Am . . . . . . . . . . . . . . . . 7

$2.1 .2 \quad$ Estrellas magnéticas, $\mathrm{Ap}$. . . . . . . . . . . . . . . 8

2.1 .3 Estrellas Hg-Mn . . . . . . . . . . . . . . . . . . . . . . . . . . . . 9

2.1 .4 Estrellas con helio anómalo . . . . . . . . . . . . . . . . . . . . . . . 10

$2.1 .5 \quad$ Estrellas $\lambda$ Bootis . . . . . . . . . . . . . . . . . . . . . . . 11

$2.1 .6 \quad$ Estrellas roAp . . . . . . . . . . . . . . . . . . . . 12

2.1 .7 Resumen . . . . . . . . . . . . . . . . . . . . . . . . . . . . . . . 12

2.2 Mapeo de abundancias químicas y manchas estelares . . . . . . . . . . . . 13

2.3 Campos magnéticos estelares . . . . . . . . . . . . . . . . . . . . . . . . . . 16

2.4 Origen del campo magnético . . . . . . . . . . . . . . . . . . . . . . . . . . . 19

2.5 Resumen . . . . . . . . . . . . . . . . . . . . . . . . . . . . . . . 22

3 Modelado de fotosferas con campos magnéticos 23

3.1 Suposiciones para el modelado de los campos magnéticos . . . . . . . . . . . 27

$3.2 \quad$ Cálculo de las conductividades eléctricas $\lambda_{i} \ldots \ldots \ldots \ldots$. . . . . . . . . . . 29

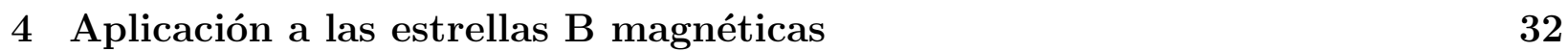

4.1 Comparación de los modelos con trabajos previos . . . . . . . . . . . . . . . 32

4.2 Comportamiento de la gravedad efectiva con el tipo espectral . . . . . . . . . 36

4.2 .1 Descripción de un modelo típico . . . . . . . . . . . . . . . 38

4.3 Efectos de la fuerza de Lorentz sobre las distribuciones de presión, densidad y temperatura . . . . . . . . . . . . . . . . . . . . 42

4.4 Comportamiento de la gravedad efectiva con la latitud magnética . . . . . . 44 
\begin{tabular}{|lll}
5 & Efectos magnéticos locales sobre las líneas de helio & 47
\end{tabular}

5.1 El átomo de helio . . . . . . . . . . . . . . . . . . . . 47

5.2 Modelado de perfiles de líneas . . . . . . . . . . . . . . . . . . 52

$5.2 .1 \quad$ Modelado de líneas en general . . . . . . . . . . . . . . . . . 52

5.2 .2 Modelado de líneas con componentes prohibidas . . . . . . . . . . 53

5.3 Efectos de la fuerza de Lorentz sobre las líneas de helio y sus anchos equivalentes 54

$5.4 \quad$ Efectos locales sobre las líneas de helio dependiendo de la latitud magnética $\quad 62$

5.5 Efectos locales sobre las magnitudes y los índices de color . . . . . . . . . . . 64

\begin{tabular}{|lll}
6 & Efectos globales y el modelo del rotador oblicuo & 66
\end{tabular}

$6.1 \quad$ Campo magnético integrado . . . . . . . . . . . . . . . . . . 66

6.2 Efectos del campo integrado $\ldots \ldots \ldots \ldots$

6.2 .1 Líneas espectrales . . . . . . . . . . . . . . . . . . . . . . . 69

6.2 .2 Magnitudes e índices de color . . . . . . . . . . . . . . . . . . 71

\begin{tabular}{lll}
\hline & Discusión & 72
\end{tabular}

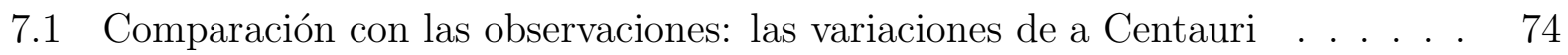

7.2 Efectos de la composición química del He . . . . . . . . . . . . . . . . . . . 79

7.2.1 Efectos combinados de la composición química y la presencia de campos magnéticos . . . . . . . . . . . . . . . . . . . 81

$\begin{array}{lll}8 \text { Difusión } & 86\end{array}$

8.1 Método de solución . . . . . . . . . . . . . . . . . . . . . . . . . . . . . . . . 91

8.2 Condiciones de borde $\ldots \ldots \ldots$. . . . . . . . . . . . . . . . . . . . . . . . . . . . . 92

8.3 Método numérico . . . . . . . . . . . . . . . . . . . . . . . . . . . . . . 92

8.4 Coeficientes difusivos y resistivos $\ldots \ldots \ldots$

$8.5 \quad$ Evaluación de las integrales de colisión, ajustes analíticos . . . . . . . . . . . 98

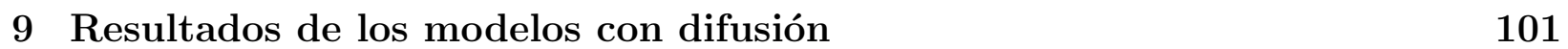

9.1 Efectos de la difusión sobre los elementos químicos. . . . . . . . . . . . . . . 101

9.2 Efectos de la latitud magnética sobre la difusión en presencia de campos magnéticos . . . . . . . . . . . . . . . . . 103

9.3 Efectos de las distintas intensidades de los campos magnéticos sobre la difusión 112

9.4 Difusión en estrellas de tipo espectral B5V . . . . . . . . . . . . . . . . . . . . . 114

\begin{tabular}{lr}
\hline 10 Conclusiones & 119
\end{tabular}

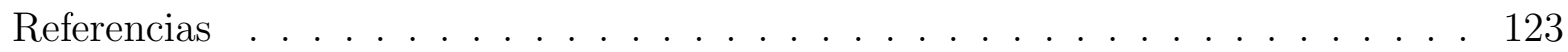

\begin{tabular}{|l|l|}
\hline A Anexo I. Los códigos & 134
\end{tabular}

A.1 Modelo de atmósfera sin campo magnético . . . . . . . . . . . . . . . . . . 134 
A.2 Gravedad efectiva . . . . . . . . . . . . . . . . . 136

A.3 Acoplamiento de la gravedad efectiva con la estructura de la atmósfera . . . 137

A.4 Normalización de las líneas espectrales y anchos equivalentes. . . . . . . . . . 138

A.5 Difusión . . . . . . . . . . . . . . . . . . . . . . . . . . . 138

\begin{tabular}{|ll}
\hline B Anexo II. Coeficientes de Splines & 140
\end{tabular}

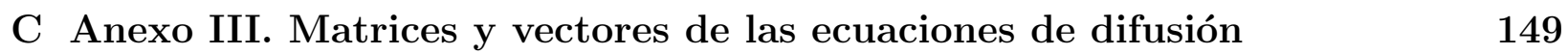

\begin{tabular}{ll}
\hline D Anexo IV. Símbolos químicos & 153
\end{tabular} 


\section{Capítulo 1}

\section{Introducción}

El campo magnético estelar es una consecuencia natural de la dinámica del plasma y está presente tanto en las estrellas de muy baja masa como en las estrellas muy masivas. Se piensa que juega un papel fundamental durante el colapso de la nube molecular y se ha detectado en objetos con diferentes fases evolutivas: en protoestrellas, en estrellas de secuencia principal y en las fases más evolucionadas (enanas blancas, supergigantes rojas, estrellas de neutrones, pulsares, etc.). El campo magnético influye significativamente en numerosos procesos físicos que operan en el interior y entorno de las estrellas, como la acreción, difusión, pérdida de masa, y turbulencia. Sin embargo, la configuración geométrica e intensidad del campo magnético depende fundamentalmente de la estructura interna de las estrellas, la velocidad de rotación y la edad Donati y Landstreet 2009.

La primera detección de un campo magnético estelar se realizó en las manchas solares Hale, 1908 y hubo que esperar varias décadas para poder detectar campos magnéticos en otras estrellas. Babcock 1947) fue el primero en medir un campo magnético organizado e intenso (de varios kiloGauss) en la estrella peculiar 78 Vir (A2p), y pasaron otros 20 años hasta, que con el descubrimiento de los pulsares, fue posible detectar campos magnéticos en estrellas de neutrones Gold 1968 con intensidades típicas del orden de los teraGauss (10 12 Gauss). Luego, a partir de los años '70, se comenzaron a reportar campos magnéticos de megaGauss a gigaGauss en algunas estrellas enanas blancas Kemp et al. 1970 y de algunos kiloGauss en las estrellas B peculiares Landstreet y Borra, 1978, Borra y Landstreet 1979, Thompson. 1983. Recién en la década del 80 fue posible medir los primeros campos magnéticos en las estrellas frías Robinson et al. 1980.

A raíz del descubrimiento de los campos magnéticos en las estrellas B peculiares, se comenzaron a realizar numerosos relevamientos que permitieron también detectar campos magnéticos en las estrellas B normales Donati, 2001, 2006, Neiner et al. 2003, Petit et al. 2008. 
Alecian et al. 2008 Henrichs et al. 2013. Una importante fracción de estrellas masivas (aproximadamente un 10\%) presentan intensos campos magnéticos de gran escala Grunhut et al. 2012, Wade y MiMeS Collaboration 2015. Muchas de estas detecciones se enmarcaron dentro del proyecto MiMeS (The magnetism in Massive Stars, Wade et al. 2016) u otros proyectos como BOB (B fields in OB stars, Fossati et al. 2015) y BinaMics (Binary and Magnetic Interactions, Alecian et al. 2015. Este último está vinculado al estudio de las propiedades magnéticas de las estrellas binarias.

Hoy en día sabemos que el campo magnético de las estrellas tipo solar, y las estrellas más frías, es altamente estructurado y variable, y está originado fundamentalmente por el efecto dínamo, es decir la transformación de la energía convectiva y de la energía mecánica por rotación en energía magnética. Este campo es responsable del calentamiento coronal y la formación de manchas de tipo solar, protuberancias, erupciones y del viento estelar. En cambio en las estrellas masivas, al no tener capas convectivas próximas a la superficie, se piensa que el campo magnético es de origen fósil Borra et al. 1982, Moss, 2001, Donati y Landstreet 2009, es decir, un campo remanente del campo acumulado o intensificado en las primeras fases de la evolución estelar. Los campos de las estrellas masivas son estructuralmente mas simples, tipo dipolar o poloidal, y frecuentemente más intensos que los campos magnéticos de las estrellas frías que presentan configuraciones multipolares o toroidales. Si bien los campos de las estrellas masivas son muy estables (Silvester et al. 2014, éstos no presentan características sistemáticas con la masa y velocidad de rotación de las estrellas Wade et al. 2000, Bagnulo et al. 2002 como las que se observan en las estrellas frías.

El descubrimiento de muchas estrellas masivas con campos magnéticos nos lleva a cuestionarnos sobre los efectos que tienen dichos campos en la estructura y evolución estelar. Este tema ha sido poco estudiado y es de gran relevancia ya que los campos magnéticos pueden alterar los procesos físicos que tienen lugar en el núcleo e interior estelar, como por ejemplo: la rotación interna, ya que son responsables del frenado magnético; los procesos de mezcla, en respuesta a las fuerzas magnéticas que actúan sobre las cargas en movimiento de los compuestos químicos que constituyen el medio; la difusión, acrecentando las diferencias en las velocidades relativas de las partículas; las pulsaciones, al inhibir el desarrollo de algunos modos de oscilación. Por otra parte, a nivel de la superficie y el entorno estelar, la fuerza de Lorentz puede generar confinamiento magnético del plasma Townsend y Owocki. 2005 y aumentar la pérdida de masa ejerciendo una aceleración extra a las partículas cargadas Lamers y Cassinelli, 1999). Como consecuencia de la influencia del campo magnético se esperan efectos radiales y transversales en la distribución de los elementos químicos produciendo heterogeneidades en las capas atmosféricas y consecuencias en la evolución de las estrellas.

Un grupo de objetos que resulta de particular interés para estudiar la influencia de los campos 
magnéticos en las propiedades de las estrellas masivas de secuencia principal son las estrellas A y B químicamente peculiares. Una característica importante de estos objetos es que son rotadores lentos y presentan intensificaciones o deficiencias de algunas especies químicas en sus atmósferas, en comparación con las características que presentan las estrellas enanas A y B normales de la misma temperatura efectiva Jaschek y Jaschek, 1987. Se piensa que las anomalías químicas están causadas por fenómenos que han tenido lugar después de la formación de la estrella, tales como difusión y campos magnéticos (Michaud, 1970). Estos procesos hacen que algunos elementos se asienten por gravedad en la atmósfera interior, mientras que otros ascienden a la superficie por efectos de la presión de radiación y de la fuerza de Lorentz. En estos objetos la baja rotación juega un papel fundamental en la estabilidad hidrodinámica, por lo que constituyen importantes laboratorios astrofísicos para estudiar los efectos magnéticos.

Nuestro objetivo es analizar el grupo de estrellas peculiares que se ubica en la parte superior del diagrama de Hertzsprung-Russell (HR) y que presentan anomalías en las intensidades de las líneas de He. Estas estrellas conocidas como He-weak (deficientes en helio) y Hestrong (enriquecidas en helio) suelen presentar variaciones fotométricas y espectroscópicas que correlacionan con la rotación de la estrella e intensos campos magnéticos. Las variaciones en las líneas de He pueden estar acompañadas por variaciones, en fase o anti-fase, de algunas líneas de otros elementos químicos, por ejemplo, del grupo del hierro (Fe) o del silicio (Si).

Es sabido que las atmósferas de estrellas B normales pueden estar contaminadas con productos del ciclo CN (carbono-nitrógeno) en la fase de secuencia principal Lyubimkov, 1984 . 1988 1989 1991, como consecuencia de los procesos de mezcla turbulentos inducidos por la rápida rotación estelar. Este proceso puede traer a la superficie productos que resultan de la fusión en los núcleos de las estrellas masivas durante las fases de la combustión del hidrógeno (es decir antes de que ocurra el primer dredge-up). Como resultado se tienen sobreabundancias de He y nitrógeno $(\mathrm{N})$, y subabundancias de carbono $(\mathrm{C})$, en la superficie de la estrella Maeder, 1987). Sin embargo, este mecanismo no es suficiente para explicar las anomalías químicas y la variabilidad que se observan en las estrellas B peculiares en He ya que estas presentan una baja rotación.

Dada la estabilidad intrínseca del campo magnético y el hecho de que la variabilidad en las líneas correlaciona con la variación de la componente longitudinal del campo magnético y con la rotación, se ha propuesto el modelo de rotador oblicuo Stibbs, 1950. En este esquema se propone que la geometría del campo magnético no es simétrica con respecto al eje de rotación de la estrella, de esta forma un observador externo ve una configuración magnética que cambia a medida que la estrella rota. Sin embargo, este modelo por si sólo no es suficiente para explicar la variabilidad observada en la intensidad de las líneas despolarizadas ni las variaciones fotométricas, lo que sugiere la existencia de distribuciones no uniformes de 
ciertos elementos químicos en la superficie estelar. Mapas de estas distribuciones químicas heterogéneas y de la estructura magnética superficial han sido realizados empleando técnicas combinadas de tomografía Doppler y de los parámetros de Stokes Kochukhov et al. 2004. Donati 2006, Rusomarov et al. 2015, o modelos de perfiles químicos superficiales Krtička et al. 2013.

Como es bien sabido la mayoría de los modelos actuales que describen la estructura interior, la atmósfera y la evolución de las estrellas desprecian los efectos magnéticos. Muy pocos trabajos han explorado los efectos de la presión magnética en la estructura de la fotosfera de las estrellas peculiares, y todos ellos se han enfocado principalmente al estudio de las estrellas Ap Carpenter. 1985, LeBlanc et al. 1994, Valyavin et al. 2004, Shulyak et al. 2007. El modelado de las distribuciones anómalas de elementos químicos (manchas) se realiza actualmente proponiendo perfiles de abundancias químicas empíricos Krtička et al. 2013 .

En este trabajo proponemos realizar un estudio riguroso de la estructura fotosférica de una estrella masiva prototipo que presenta anomalías en He, analizando los efectos de la presión magnética en la estructura de la fotosfera. La presencia de un campo magnético que no está libre de fuerzas producirá cambios en las distribuciones de presión y densidad y, consecuentemente, cambios en la opacidad de las líneas y del continuo. Se discutirán los efectos de la componente radial de la fuerza de Lorentz en líneas de He a nivel local y se considerará la contribución integrada del espectro de línea sobre el hemisferio visible de la estrella. A tal fin se resuelve rigurosamente la ecuación de transporte radiativo y la ecuación de equilibrio hidrostático. Luego, considerando el modelo de rotador oblicuo se discute el aspecto que tendrán los perfiles de líneas con la fase de rotación de la estrella y los efectos de una distribución anómala de He en la superficie. Para este último caso se resuelven las ecuaciones de difusión atómica para la atmósfera estelar en ausencia y presencia de un campo magnético. Finalmente se discuten los resultados en el contexto de algunas observaciones.

El presente trabajo está organizado de la siguiente manera:

En el capítulo $\$ 2$ se hace una reseña de las propiedades de las estrellas peculiares y las propiedades de los campos magnéticos de las mismas. En el capítulo \$3 presentamos el cálculo de la estructura de la atmósfera estelar en presencia de un campo magnético dipolar. En el capítulo 4 modelamos la magnetosfera de las estrellas de tipo espectral B. En el capítulo \$5analizamos cómo la fuerza de Lorentz, y por ende también el campo magnético, modifican a los perfiles de las líneas de He a nivel local. En el capítulo estudiamos los efectos globales al considerar un rotador magnético oblicuo. En el capítulo 77 realizamos una discusión de los resultados obtenidos en los capítulos anteriores y el alcance de los mismos al ser comparados con las observaciones. En los capítulos $\$ 8$ y $\$ 9$ se modelan los procesos de la 
difusión atómica a nivel de la atmósfera para explicar posibles intensificaciones en ausencia y presencia de un campo magnético y el perfil químico superficial que resulta. A igual que en el caso anterior se discutirá el efecto local del campo magnético en la distribución anómala de elementos químicos. Finalmente, en el capítulo \$10 se dan las conclusiones del presente trabajo.

En forma de anexo, en $\oiint \mathrm{A}$, se detallan los códigos utilizados en este trabajo. En $\S \mathrm{B}$, se tabulan los coeficientes de Spline necesarios para modelar los procesos de difusión. En đC. se da el esquema de matrices y vectores involucrados en los procesos de difusión. En $₫ \mathrm{D}$, se da un glosario de los elementos químicos que aparecen mencionados en este trabajo. 


\section{Capítulo 2}

\section{Estrellas químicamente peculiares}

\subsection{Estrellas A y B químicamente peculiares}

Entre las estrellas de tipo espectral A y B, existe un subgrupo que presenta anomalías en las intensidades de algunas líneas espectrales, como ser las de $\mathrm{He}$, mercurio (Hg), manganeso $(\mathrm{Mn})$, zinc $(\mathrm{Zn})$, estroncio $(\mathrm{Sr})$, bario $(\mathrm{Ba})$, zirconio $(\mathrm{Zr})$, tierras raras, etc. Estas líneas, en absorción, se ven más intensas o más débiles que las de la mayoría de las estrellas de su mismo tipo espectral (Jaschek y Jaschek 1987). Esto se atribuye a una deficiencia o a una sobreabundancia de las especies químicas correspondientes a las mencionadas líneas. En algunos casos se llegan a observar variaciones en las intensidades de las mismas.

Otra característica llamativa de este grupo de estrellas, al que se denomina $C P$, por su sigla en inglés chemically peculiar stars, es que rotan a una velocidad más baja que la de sus contrapartes normales y en su mayoría presentan campos magnéticos intensos. En un principio, se había observado la presencia de campos magnéticos sólo en algunas de las estrellas CP Borra et al. 1982 pero en la última década, fundamentalmente con la aparición de relevamientos como MiMeS, se ha encontrado que casi todas las estrellas CP poseen campos magnéticos.

Desde el descubrimiento de las estrellas CP, su clasificación ha ido variando, llegando a reconocerse hasta unas doce subclases. En la actualidad se adopta la clasificación realizada por Preston 1974, a saber:

- CP1: Estrellas que presentan líneas metálicas, o estrellas A metálicas, también denominadas Am.

- CP2: Estrellas magnéticas, llamadas Ap, que se subdividen a su vez en tres grupos, las de $\mathrm{Si}$, las de cromo-europio (Cr-Eu), y las de $\mathrm{Sr}$. 
- CP3: Estrellas de mercurio-manganeso (Hg-Mn).

- CP4: Estrellas con abundancia de helio anómala. Son las denominadas deficientes en He (He-weak) y presentan líneas débiles de He. En muchos casos las intensidades de las líneas varían en forma periódica.

Relacionadas con este último grupo, pero fuera de la clasificación de Preston, hay un grupo de estrellas peculiares en He, en las cuales sus líneas de He se ven intensificadas, presentando también variaciones periódicas en su intensidad. A este grupo de estrellas se las denomina sobreabundantes en He (He-strong).

Dos casos particulares, son las estrellas a Centauri y HD 5737, en donde las variaciones de las líneas de He son tan intensas, que por momentos podrían clasificarse como pertenecientes a la clase de las He-weak y en otros como pertenecientes a las He-strong.

En general, se piensa que la composición química peculiar que es observada en la superficie de estas estrellas está causada por procesos que han tenido lugar después de la formación de la estrella, tales como difusión o campos magnéticos. Estos procesos favorecen a que algunos elementos se asienten en las capas inferiores de la atmósfera, mientras que otros elementos asciendan desde el interior hacia la superficie, provocando las particularidades espectrales observadas. Es decir que los elementos químicos se han redistribuido ya que se supone que el interior de la estrella, así como la estrella en su conjunto, poseen una abundancia química normal que refleja la composición de la nube de gas de la cual se formó.

\subsubsection{Estrellas A metálicas, Am}

Son estrellas cuyo espectro se corresponde con estrellas de tipo espectral A pero presentan intensificaciones en varias de las líneas metálicas.

Las primeras en observar estrellas con estas peculiaridades fueron las clasificadoras de Harvard, Antonia Maury y Annie J. Cannon a principios del siglo XX. Ellas observaron que un grupo de estrellas, entre las que se encontraban $\delta$ Nor, $\tau$ UMa y $\tau^{3}$ Eri, presentaban la línea $\mathrm{K}$ del calcio ionizado (Ca II) débil, pero aparecían líneas metálicas intensas, similares a las encontradas en las estrellas F.

La terminología, Am, se debe a John Titus y W. W. Morgan quienes la utilizaron al clasificar estrellas A en el cúmulo Hyades Titus y Morgan 1940. Formalmente, estas estrellas aparecen por primera vez como estrellas clase Am en el Atlas MKK (Morgan et al. 1943. El patrón de abundancias de las estrellas Am muestra deficiencias en calcio (Ca), itrio (Y) y escandio (Sc), mientras que los elementos del pico de Fe y elementos más pesados aparecen como sobreabundantes. Desde el punto de vista fotométrico, para una misma temperatura efectiva, las estrellas Am aparecen como más rojas que las estrellas A normales. 


\subsubsection{Estrellas magnéticas, Ap}

Fueron las primeras estrellas peculiares descubiertas. A diferencia de las estrellas Am, que presentan una fotosfera con sobreabundancia en casi todos los elementos químicos pesados, las estrellas Ap sólo presentan sobreabundancias en ciertos elementos particulares. Por otro lado, teniendo en cuenta sus temperaturas efectivas, son mayormente estrellas de tipo espectral B tardío.

Maury y Pickering 1897 clasificaron y analizaron unas 12 estrellas Ap observando el doblete de Si II. En principio, como se puede ver en el relevamiento de Cannon y Pickering 1901, se definieron dos tipos de estrellas Ap. Una de las clases contiene a las estrellas con sobreabundancia en Si (entre estas, $\nu$ For, $\tau^{9}$ Eri y $\alpha$ Dor) y la otra agrupa a las estrellas con sobreabundancias en $\mathrm{Sr}$ (incluyendo $\xi$ Phe, $\theta^{1}$ Mic e $\iota$ Phe). Más adelante, Cannon y Pickering 1912a b), agregaron 25 estrellas más al listado de esta clase.

Entre 1931 y 1933, William W. Morgan realizó el primer estudio sistemático de estrellas Ap. Él identificó cinco grupos, a saber: Mn II, $\lambda 4200$, Eu II, Cr II y Sr II, aunque las estrellas de un grupo también presentaban las características del otro.

Posteriormente, Mercedes Corbalán-Jaschek y Carlos Jaschek Jaschek y Jaschek, 1958, definieron seis grupos: $\lambda 4200, \mathrm{Mn}, \mathrm{Si}, \mathrm{Si}-\mathrm{Cr}-\mathrm{Eu}, \mathrm{Eu}-\mathrm{Cr}-\mathrm{Sr}$ y Sr, aunque luego Bidelman 1962a determinó que la línea $\lambda 4200$ correspondía a una línea de alta excitación del Si II, con lo cual este grupo pasó a estar incluido en el grupo del Si. Por otra parte, Osawa 1965 identificó 16 clases de peculiaridades en las estrellas Ap. Esta clasificación resulta demasiado detallada, incluyendo la separación de tipos espectrales obtenidos a partir de las líneas $\mathrm{K}$ del Ca II y de hidrógeno (H). En el mismo sentido, Garrison y Gray 1994 realizaron una clasificación con una notación similar, pero considerando las intensidades de las líneas de He I.

Una de las características de las estrellas Ap es que son variables espectroscópicas en las cuales la variación de la intensidad de las líneas peculiares, coincide con el período de rotación de la estrella Floquet, 1979. Esto sugiere que la sobreabundancia de un dado elemento químico no ocurre en toda la superficie de la estrella, sino en forma de manchas. De esta forma, las variaciones de la intensidad de una dada línea, se pueden atribuir a la existencia de una mancha del elemento químico asociado a esa línea, en un determinado hemisferio, mientras que las variaciones de otras líneas podrían indicar la presencia de una o varias manchas de otros elementos químicos. En este sentido, las observaciones permiten realizar mapas de estas manchas Khokhlova et al. 1991, Lehmann et al. 2007, Polosukhina et al. 2014 Rusomarov et al. 2015, como veremos en la sección 2.2 El origen de estas manchas estaría asociado con la presencia de campos magnéticos. En las estrellas Ap se han observado campos magnéticos que van desde unos cientos a unos pocos miles de Gauss Aurière et al. 
2007a bochukhov, 2014.

\subsubsection{Estrellas $\mathrm{Hg}-\mathrm{Mn}$}

Las estrellas de Hg-Mn son estrellas de tipo espectral entre B7 y B9, y clase de luminosidad entre V y III. Si las comparamos con las estrellas normales del mismo tipo espectral, observamos que las velocidades de rotación de las estrellas peculiares de Hg-Mn son menores. El rasgo fundamental que caracteriza a este tipo de estrellas es la gran abundancia de $\mathrm{Hg}$ en su atmósfera, que en algunos casos alcanza los 5 dex, así como también la gran cantidad de Mn presente que llega a ser de más de 3 dex. Esto se pone de manifiesto por la presencia de líneas muy intensas de $\mathrm{Hg}$ y de Mn. Las líneas que más fácilmente se observan son las del Hg II $\lambda 3984$ y las del Mn II, $\lambda 4136, \lambda 4206$ y $\lambda 4252$. Hay estrellas en las que el Hg II no se observa y se las denominan estrellas de Mn Morgan, 1931), así como también hay casos de estrellas donde el Mn es normal y el Hg aparece sobreintensificado Bidelman y Corliss. 1962 Bidelman, 1962b.

La abundancia de los distintos elementos químicos varía de una estrella a otra. En general son deficientes en elementos livianos como el He, así como también en $\mathrm{C}, \mathrm{N}$, oxígeno $(\mathrm{O})$ y azufre $(\mathrm{S})$, aunque en algunas estrellas el $\mathrm{C}$ presenta sobreabundancia. La abundancia de Si es normal y la del fósforo $(\mathrm{P})$, cuando se observa es sobreabundante. En general, cuando se encuentran presentes, los elementos pesados son sobreabundantes, como los casos del neodimio $(\mathrm{Nd})$, praseodimio $(\mathrm{Pr})$, oro $(\mathrm{Au})$, xenón $(\mathrm{Xe})$, galio $(\mathrm{Ga})$, y platino $(\mathrm{Pt})$. La abundancia de Fe difiere en cada estrella, mientras que en algunas es deficiente, en otras aparece como sobreabundante.

Se han esbozado varias teorías para tratar de explicar las anomalías químicas observadas, pero los resultados obtenidos no alcanzan a explicarlas completamente. Incluso, en algunos casos, ni la difusión radiativa es suficiente para explicar estas anomalías (Dolk et al. 2003. Originalmente estas estrellas fueron consideradas como no magnéticas, pero recientemente, se han detectado campos magnéticos de hasta 1000 Gauss, tal es el caso de las estrellas 74 Aqr y $\chi$ Lup (Mathys y Hubrig, 1995).

Dado que algunas de estas estrellas muestran variaciones en sus espectros, por ejemplo en la línea Hg II $\lambda 3984$, esta observación se interpreta como debida a la existencia de una distribución no uniforme de las abundancias en sus superficies, que al igual que las Ap, podría estar relacionada a la presencia de campos magnéticos. 


\subsubsection{Estrellas con helio anómalo}

Se las divide en dos clases, las ricas o intensas en He (He-strong) y las débiles o deficientes en He (He-weak). En la literatura, en algunos casos, también aparecen como He-rich y He-poor, respectivamente.

\section{He-weak}

Estas son estrellas de tipo espectral B, en general B3 y más tardías, en las que las líneas de He son peculiarmente débiles y son clasificadas según la intensidad de sus líneas de H. Osawa 1965 utiliza las líneas de He para clasificar a las He-weak, y este criterio es continuado por Garrison y Gray 1994. Algunas de estas estrellas presentan también, líneas metálicas con intensidades inusuales.

Estas estrellas fueron descubiertas cuando, analizando relaciones entre el espectro y los índices de color, se encontraron discrepancias en los índices U-B y B-V, los que resultaban más azules de lo esperado para el tipo espectral asignado Sharpless, 1952a bar Garrison. 1973. Este fue uno de los criterios utilizados para encontrar estrellas He-weak Bernacca. 1968 Jaschek et al. 1969 .

Las estrellas He-weak suelen presentar también algunas características comunes con las estrellas Ap y Hg-Mn, y se las suele agrupar en tres subclases: las estrellas de Silicio (Si), las estrellas de Fósforo-Galio (PGa), y las estrellas de Estroncio-Titanio (SrTi).

Las estrellas He-weak de Si están caracterizadas por poseer un mayor número de líneas de $\mathrm{Si}$, pero con temperaturas más altas que las Ap clásicas. Como no son muy diferentes a las Ap podrían pensarse que son la contraparte caliente de las estrellas Ap de Si. Las estrellas He-weak de SrTi, presentan una mayor cantidad de Sr y Ti, pero poseen tipos espectrales más tempranos que las estrellas Ap clásicas de Sr.

En muchos casos, las líneas de He I de las estrellas He-weak presentan perfiles peculiares. Tal es el caso de las líneas de He I de 3 Sco que tienen un perfil triangular, en especial He I

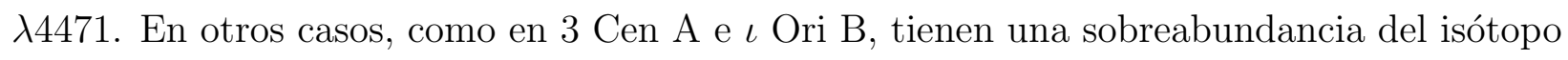
${ }^{3} \mathrm{He}$, y esa sería la causa de los perfiles inusuales de He I Sargent y Jugaku, 1961, Dworetsky. 1973 Hartoog y Cowley, 1979.

\section{He-strong}

Las estrellas He-strong son estrellas de tipo espectral B tempranas (B3 y más tempranas) que poseen líneas extremadamente intensas de helio neutro (He I). La estrella típica de esta clase es $\sigma$ Ori E, que es una estrella del cúmulo $\sigma$ Ori. Otra estrella de esta clase es $\delta$ Ori C. Algunas de las estrellas de este grupo son variables fotométricas y espectroscópicas, mostrando en algunos casos, grandes cambios en las intensidades de las líneas de He I. El 
primero en descubrir la variabilidad de las estrellas He-strong fue Bidelman 1965) quien encontró que la estrella a Cen (HR 5378, HD 125823) tenía líneas de He I intensas que variaban entre los grupos He-strong y He-weak.

Otra estrella que muestra variaciones en la intensidad de las líneas de He I es HD 184927, aunque en este caso entre He-strong y He-normal Bond y Levato, 1976, Levato y Malaroda. 1979. En su trabajo, Levato y Malaroda 1979 encontraron que el período de variación es de 9.536 días. Mucho después, Wade et al. 1997 midieron la variabilidad del campo magnético de HD 184927, entre -0.7 y 1.8 kG, y que esas variaciones tienen un período muy cercano al encontrado por Levato y Malaroda 1979.

Las estrellas He-strong, pueden presentar o no campos magnéticos, pero en el caso de poseerlos, estos son más intensos que los observados en las estrellas He-weak Borra et al. 1983, y en promedio son unas tres veces más grandes que los campos magnéticos de las Ap Bohlender et al. 1987.

Recientemente, siguiendo la clasificación de Preston 1974, Swetlana Hubrig propuso agrupar a las estrellas He-weak como CP4a y a las estrellas He-strong como CP4b. Una excepción son los casos de las estrellas HD 5737 y HD 125823 (a Cen). Como ya mencionamos, estas dos estrellas muestran comportamientos típicos de estrellas He-weak y He-strong, alternándose entre un subgrupo y otro. Debido a este comportamiento, Hunger y Groote 1999 agruparon a estas dos estrellas en un grupo intermedio, al cual Hubrig ha propuesto denominar como subgrupo CP4ab.

\subsubsection{Estrellas $\lambda$ Bootis}

Fuera de la clasificación de Preston, también existe otro grupo de estrellas CP, denominadas $\lambda$ Bootis. Son estrellas de tipo espectral A, de población I y con líneas de metales débiles. El descubrimiento de estas estrellas se remonta a 1943, cuando la estrella arquetípica de la clase, $\lambda$ Boo, fue incluida en el catálogo An atlas of stellar spectra, with an outline of spectral classification Morgan et al. 1943. Se la clasificó como una A0 con líneas metálicas débiles, donde sólo se veían la línea K y la serie de Balmer. Además, la línea de Mg II $\lambda 4481$, aparecía inusualmente débil.

Más adelante, Gray 1997 dio una definición sin ambigüedades para la clasificación de estos objetos. En síntesis, son estrellas A y F tempranas con un rango de tipos espectrales entre las B9.5 y las F0. Tienen una relación Mg II $\lambda 4481$ / Fe II $\lambda 4383$ más chica que la de las estrellas A normales. Son claramente deficientes en metales y poseen líneas de $\mathrm{H}$ anchas. Por último, las velocidades de rotación, son similares a las de las estrellas de tipo A normales. 


\subsubsection{Estrellas roAp}

También, por fuera de la clasificación de Preston, existe otro grupo de estrellas químicamente peculiares, las denominadas roAp (rapidilly oscilating $A p$ ). Son estrellas Ap que poseen variaciones fotométricas rápidas y variaciones en sus velocidades radiales. Estas variaciones son producto de las pulsaciones que sufre la estrella, de allí el nombre. Los períodos de estas variaciones van desde 5 a 23 minutos.

La primera estrella que se descubrió que poseía esta peculiaridad fue la estrella HD 101065, conocida como estrella de Przybylski Kurtz, 1978). En ese trabajo, Donald Kurtz observó variaciones entre 10 y 20 milimagnitudes con un período de 12.15 minutos.

En este tipo de estrellas, las pulsaciones son no radiales, y el comportamiento de las pulsaciones se explica mediante el modelo del pulsador oblicuo, en donde el eje de simetría de las pulsaciones está alineado con el eje magnético, el cual difiere también del eje de rotación en un determinado ángulo.

\subsubsection{Resumen}

Basados en el trabajo de Smith 1996, en la tabla 2.1 mostramos un pequeño resumen de la clasificación de las estrellas químicamente peculiares. En la primera columna se da el nombre clásico con que se conoce al grupo de estrellas. La segunda columna nos da la clasificación de Preston 1974. La tercera columna indica las características de las líneas que se utilizan para clasificar a las estrellas dentro de uno u otro grupo. En las dos últimas columnas se listan el rango de tipos espectrales y el rango de temperaturas de cada grupo, respectivamente.

Tabla 2.1: Clasificación de las estrellas CP.

\begin{tabular}{|l|c|l|c|c||}
\hline $\begin{array}{l}\text { Nombre } \\
\text { clásico }\end{array}$ & $\begin{array}{c}\text { Grupo de } \\
\text { Preston }\end{array}$ & Líneas de clasificación & $\begin{array}{c}\text { Tipo } \\
\text { espectral }\end{array}$ & $\begin{array}{c}\text { Temperaturas } \\
\text { (K) }\end{array}$ \\
\hline \hline Am-Fm & CP1 & $\begin{array}{l}\text { Ca II y Sc II tenues } \\
\text { Metales intensas }\end{array}$ & A0 - F4 & $7000-10000$ \\
\hline Bp-Ap & CP2 & Sr, Cr, Eu y Si intensas & B6 - F4 & $7000-16000$ \\
\hline Hg-Mn & CP3 & Hg II y Mn II intensas & B6 - A0 & $10500-16000$ \\
\hline He-weak & CP4 & He I tenues & B2 - B8 & $14000-20000$ \\
\hline He-strong & & He I intensas & B2 & $20000-25000$ \\
\hline$\lambda$ Boo & & Mg II y metales tenues & A0 - F0 & $7000-9000$ \\
\hline
\end{tabular}

En la figura 2.1. mostramos una serie de histogramas, extraídos del trabajo de Smith 1996, correspondientes a cada uno a los distintos grupos de estrellas químicamente peculiares, indicando la distribución de los tipos espectrales de las estrellas de cada grupo. Allí vemos 
claramente el rango de tipos espectrales que abarca cada clase de estrella, y cuales son los tipos espectrales preponderantes.

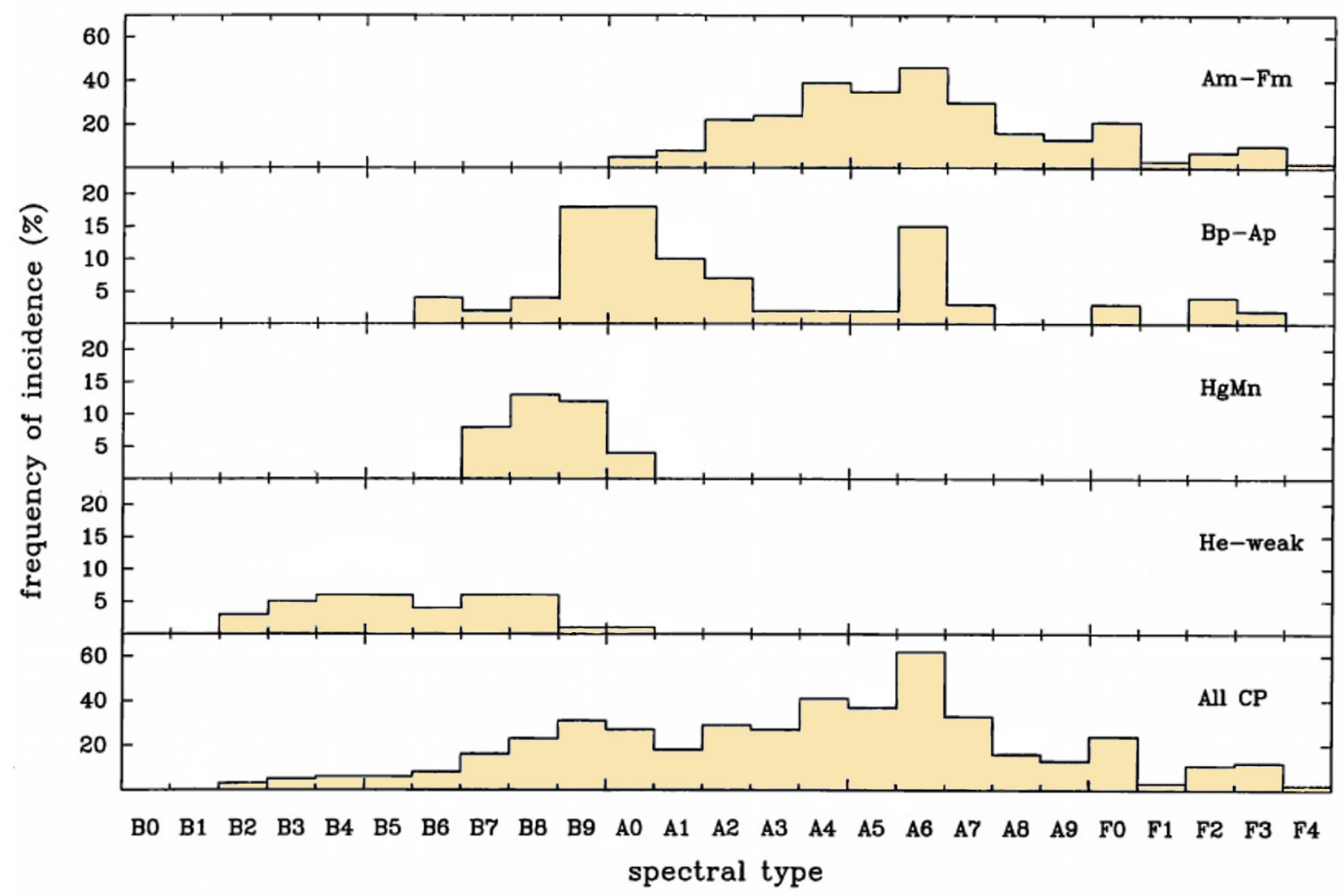

Figura 2.1: Histograma con la frecuencia de incidencia de estrellas CP en cada tipo espectral. (Gráfico adaptado de Smith 1996 .

\subsection{Mapeo de abundancias químicas y manchas este- lares}

A lo largo de los últimos 30 años, han aparecido varios trabajos que, a partir de observaciones sistemáticas y empleando diferentes técnicas, han determinado mapas de las abundancias de los diferentes elementos químicos en la superficie de varias estrellas CP. Además, muchos de estos trabajos incluyen mapas de los campos magnéticos en estas estrellas. Si bien estos trabajos indican que hay una relación entre la presencia de campos magnéticos y manchas, no queda claro cuales son los mecanismos que inducen a la formación de las manchas.

La técnica más utilizada últimamente para realizar los mapeos de abundancias superficiales es la tomografía Doppler (Doppler imaging). Básicamente, consiste en tomar espectros de alta resolución de la estrella en distintas fases rotacionales. A partir del corrimiento Doppler de las líneas de los distintos elementos químicos, se reconstruye la distribución de las abundancias sobre la superficie de la estrella. Además, si se mide la polarización, se puede reconstruir 
la distribución de la componente longitudinal del campo magnético sobre la superficie de la estrella.

Varias son las estrellas que se han estudiado por esta técnica. Por ejemplo, $\varepsilon$ Ursae Majoris ( $\varepsilon$ UMa, HD 112185), $\alpha^{2}$ Canum Venaticorum ( $\alpha^{2}$ CVn, HD 112413), $\theta$ Aurigae ( $\theta$ Aur), CU Vir, $\chi$ Ser, 21 Per (HD 18269), 53 Cam, HD 215441, HD 37776, HD 12098, HD 60435, HD 83368, HD 3980, HR 7224, HD 24712. En ellas se han realizado mapeos de abundancias de $\mathrm{He}, \mathrm{O}, \mathrm{Fe}, \mathrm{Si}, \mathrm{Cr}, \mathrm{Eu}, \mathrm{Sr}, \mathrm{Ti}, \mathrm{Mn}$, litio (Li), Pr, sodio (Na), y Nd. En algunas también se han hecho mapeos de sus campos magnéticos.

Estos estudios han sido realizados entre otros por: Wehlau et al. 1982, 1991); Goncharskii et al. 1983; ;hokhlova y Pavlova 1984; Khokhlova et al. 1986, 1991, 1997, 1998, 2000; Lehmann et al. 2007); Lueftinger 2014); Polosukhina et al. 2014) y Rusomarov et al. 2015.

Por ejemplo, Rusomarov et al. 2015, utilizando la técnica de tomografía Doppler y los parámetros de Stokes, estudiaron la estrella Ap HD 24712. Ellos realizaron mapas de la distribución de abundancias de Fe, Nd III y Na, los cuales podemos ver en la figura 2.2 . Además, determinaron la distribución del módulo del campo magnético, sus componentes radial y horizontal, y la orientación del campo como se muestra en la figura 2.3 .
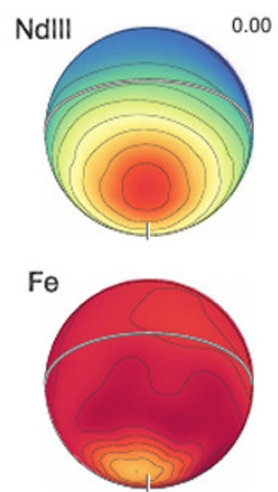

$\mathrm{Na}$

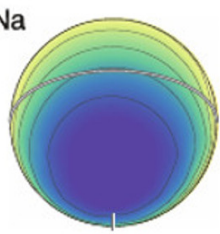

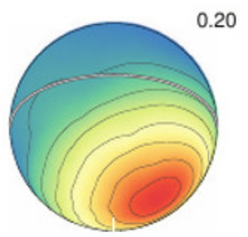
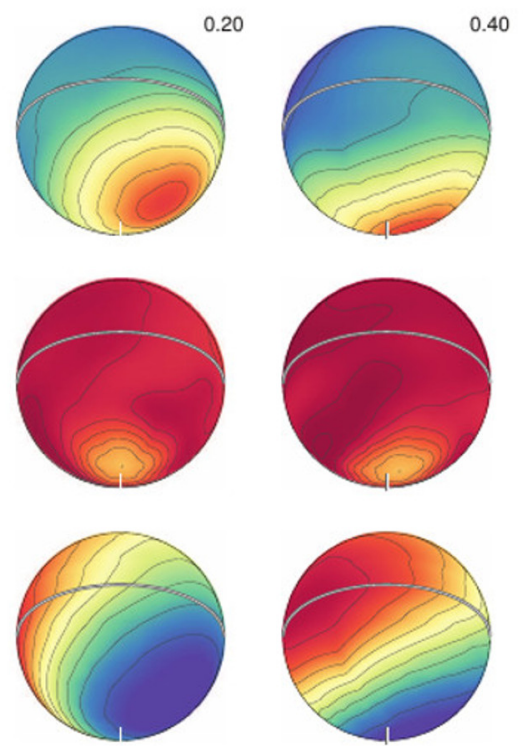
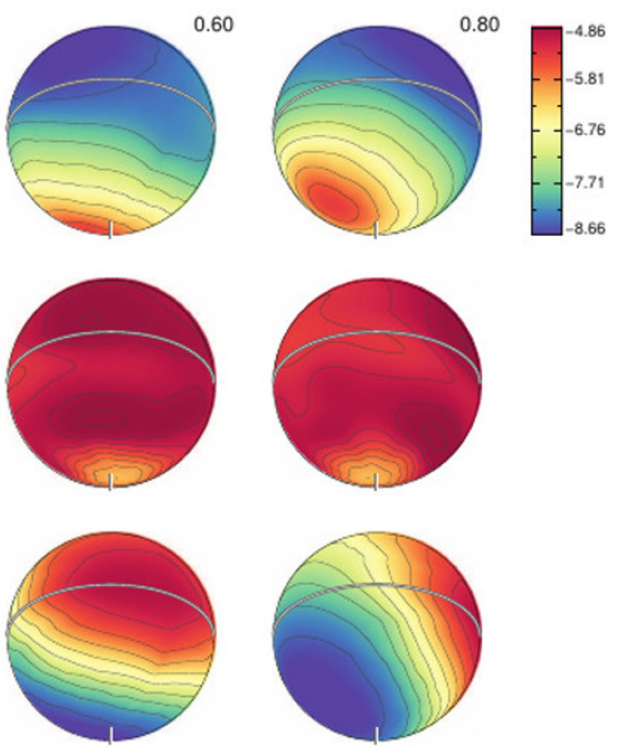

Figura 2.2: Gráfico del trabajo de Rusomarov et al. 2015, en donde se muestra la distribución de las abundancia de Nd III (arriba), Fe (centro) y Na (abajo), para 5 fases rotacionales distintas.

En otro sentido, Krtička et al. 2013, a partir de mapas de la distribución de He y Si, realizan modelos teóricos para explicar el comportamiento de la curva de luz en el rango visual y ultravioleta de la estrella peculiar HD 64740. Ellos consideran un grillado sobre la superficie de la estrella, y en cada punto de ese grillado, resuelven un modelo de atmósfera 

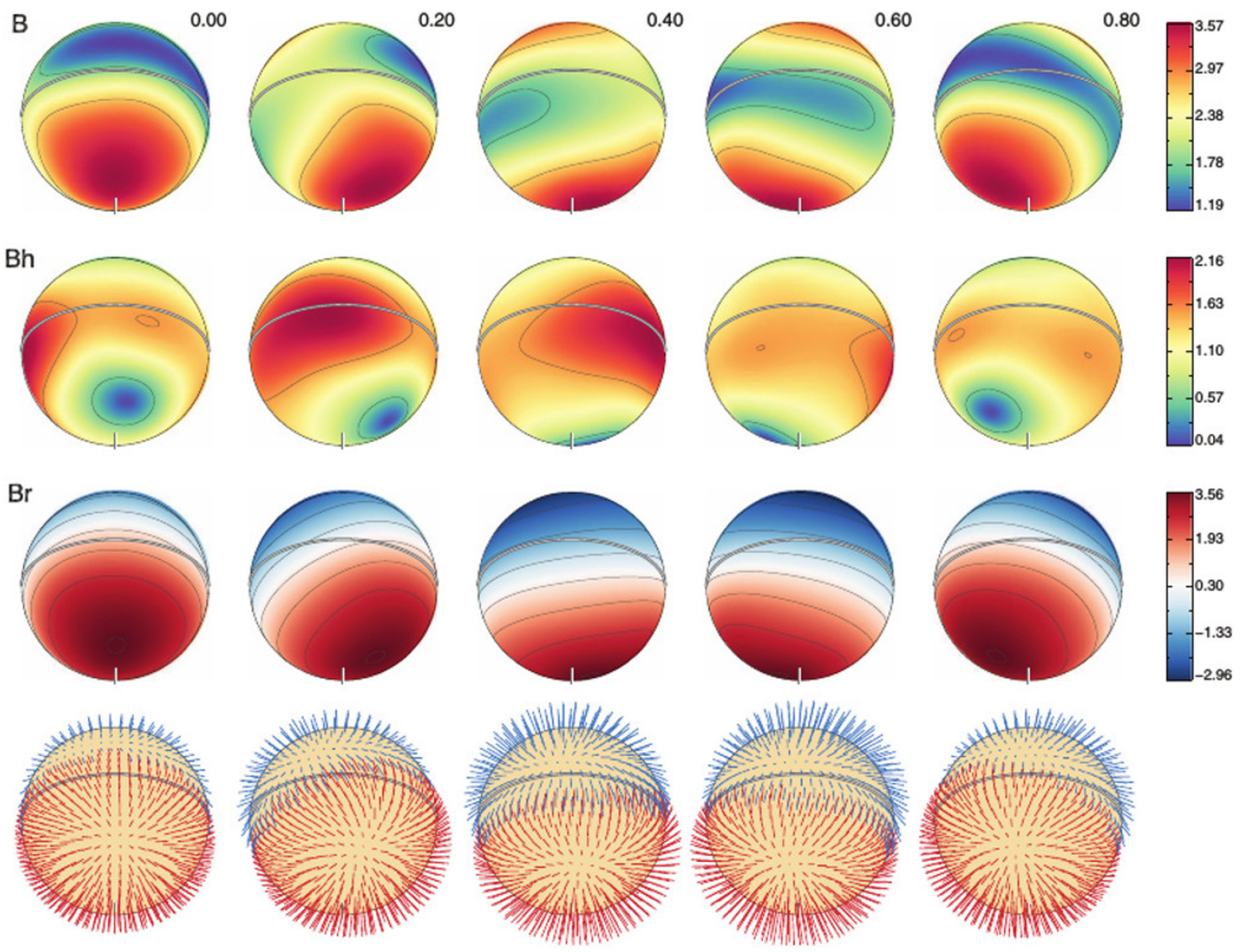

Figura 2.3: Gráfico del trabajo de Rusomarov et al. 2015, en donde se muestra en la primera fila, la distribución del módulo del campo magnético, en la segunda, su componente horizontal, en la tercera, su componente radial, mientras que en la cuarta fila se muestra la distribución de orientaciones del campo.

con las correspondientes abundancias de He y Si asignadas a ese punto. Luego integran los resultados obtenidos sobre el disco visible de la estrella en cada fase rotacional, de forma tal de poder comparar los resultados con las observaciones y verificar que el mapeo de abundancias explique las variaciones observadas en la curva de luz. Si bien este trabajo prueba que la existencia de zonas de diferentes abundancias podría justificar las variaciones observadas, los autores no indican cual sería la causa que origina las anomalías químicas de dichas zonas.

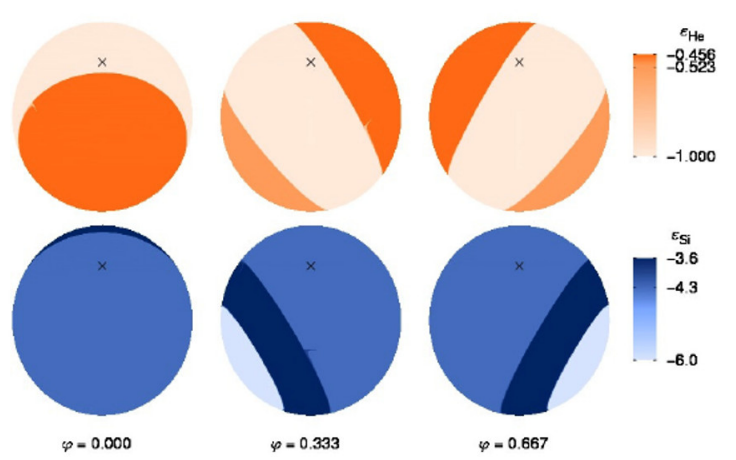

Figura 2.4: Gráfico del trabajo de Krtička et al. 2013, en donde se muestra en la parte superior la distribución superficial de He, y en la parte inferior, la de Si, que utilizaron como base para sus modelos. 
En la figura 2.4. mostramos un gráfico del trabajo de Krtička et al. 2013, en donde se muestra en la parte superior la distribución de He, y en la inferior la distribución de Si.

Generalmente se cree que la distribución no homogénea de los elementos químicos en la superficie de las estrellas Ap y Bp es consecuencia del efecto del campo magnético. Hay dos teorías que explicarían la formación de manchas de distintas abundancias en la superficie estelar. Una es la teoría de difusión bajo efectos de la gravedad y la presión de radiación Michaud 1970, 1980, Vauclair y Vauclair 1982, y la otra es la teoría de acreción selectiva de elementos a partir del medio interestelar (Havnes y Conti, 1971). Ambas teorías involucran la presencia de campos magnéticos.

\subsection{Campos magnéticos estelares}

Como mencionamos en la introducción, las estrellas masivas poseen campos magnéticos y sus propiedades dependen de nuestra capacidad de poder detectarlos y eventualmente cuantificarlos. Hoy en día se conoce que la mayoría de las estrellas $\mathrm{CP}$ poseen intensos campos magnéticos, e incluso se han podido realizar mapeos de dichos campos en la superficie de algunas estrellas como mostramos anteriormente en la figura 2.3 .

La detección de los campos magnéticos se realiza a partir de la observación del efecto Zeeman en las líneas espectrales, el cual produce cambios en el perfil de absorción de la línea, como a su vez, en la polarización lineal y/o circular. La medición de la separación o ensanchamiento de los perfiles de las líneas espectrales permite obtener el campo magnético superficial medio $B_{s}$, el cual está definido por,

$$
B_{s}=\frac{\int|\boldsymbol{B}| I d A}{\int I d A},
$$

donde $|\boldsymbol{B}|$ es el módulo de la intensidad del campo magnético local, $I$ es el brillo superficial local, y $d A$ es el elemento de área sobre la superficie. Las integraciones se efectúan sobre todo el disco visible de la estrella.

El campo magnético será medible sólo si la estrella posee líneas espectrales angostas y bien definidas $(v \operatorname{sen} i<10 \mathrm{~km} / \mathrm{s})$ y la intensidad del campo es lo suficientemente grande, $|\boldsymbol{B}|$ $>1000$ Gauss, aunque este límite de detección ha ido disminuyendo en los últimos años Babcock 1960b, Preston, 1969bal, c, 1971, Wolff y Wolff, 1970, Robinson et al. 1980 gracias al desarrollo de nuevo instrumental, más sensible y con mayor resolución espectral.

La medición de la polarización circular permite obtener el campo magnético longitudinal (o efectivo) medio $B_{l}$, definido por, 


$$
B_{l}=\frac{\int B \cos \gamma I d A}{\int I d A}
$$

donde $\gamma$ es el ángulo entre la dirección de la línea de fuerza y la dirección de la línea de la visual. En la figura 2.5. mostramos gráficamente este ángulo $\gamma$. Dada la dependencia con $\gamma$, $B_{l}$ (o $H_{l}$ ) depende más de la geometría del campo magnético que de su intensidad $B_{s}$.

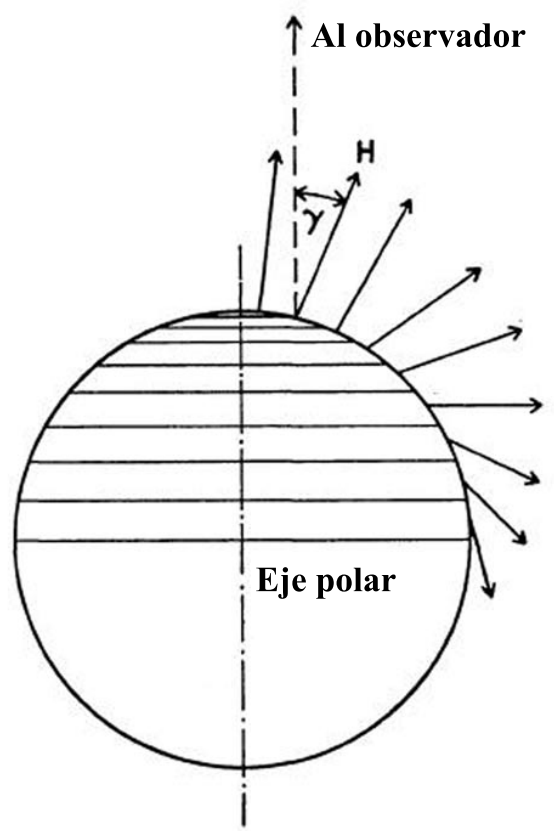

Figura 2.5: Variación de la dirección y de la intensidad del campo magnético con la latitud sobre la superficie de una estrella. El vector del campo $\boldsymbol{H}$ y la línea de la visual forman un ángulo $\gamma$ Babcock 1947.

La componente transversal del campo magnético resulta de medir la polarización lineal. En la práctica, la determinación es difícil ya que los valores de la polarización lineal son pequeños. A pesar de esta dificultad se han realizado algunas mediciones como las que encontramos en los trabajos de Kodaira y Unno 1969 y Borra y Vaughan 1976.

En particular en lo que se refiere a las estrellas CP, desde que Babcock 1947) detectó el primer campo magnético en la estrella Ap 78 Vir, en los siguientes 30 años se detectaron unas 200 estrellas CP con campos magnéticos, la mayoría de ellas pertenecientes al grupo de las Ap, de las cuales unas 50 estrellas habían sido estudiadas en detalle, principalmente por Babcock. Hoy en día, el número de estrellas $\mathrm{CP}$ con campos magnéticos aumenta constantemente donde la mayoría de estos campos han sido detectados estudiando las líneas de la serie de Balmer.

En general, los campos magnéticos longitudinales observados son variables en el tiempo. La mayoría de las estrellas CP poseen períodos de variación de su campo magnético entre 1 y 10 días, siendo el valor medio de los períodos cercano a los 3 días. 
También se ha observado que la variación temporal de la componente longitudinal de estas estrellas tiene forma sinusoidal y el período de la variación magnética coincide, o casi coincide, con el período de la rotación. A modo de ejemplo, en la figura 2.6 mostramos las mediciones del campo magnético longitudinal de la estrella HD 184927, en donde se ve claramente el comportamiento sinusoidal antes mencionado Yakunin et al. 2015. En general, el valor de $B_{s}$ es dos o tres veces mayor que el valor más grande medido de $B_{l}$, y varía con el mismo período que $B_{l}$.

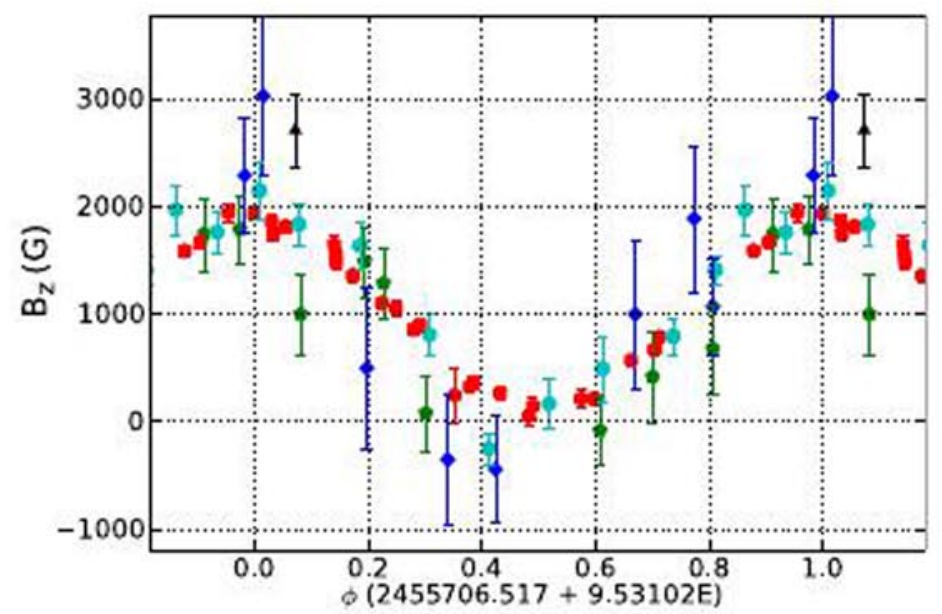

Uwo Polarimeter + Palomar 200 -inch $\left(\mathrm{H}_{3}\right)$

UWo Polarimeter + UWO $1.2-\mathrm{m}(\mathrm{H} \beta)$

Uwo Polarimeter + UH 88-inch $(\mathrm{H}, 3)$

ESPaDONS + CFHT $(\mathrm{H} \alpha)$

dimaPol + DAO 1.8.m $(\mathrm{H}, 3)$

Figura 2.6: Variación del campo magnético longitudinal con la fase rotacional obtenida a partir de las líneas del H, con distintos instrumentos Yakunin et al. 2015.

Como consecuencia de estas variaciones sinusoidales de la componente longitudinal del campo magnético, los anchos equivalentes de los perfiles de línea también varían de la misma manera, como puede apreciarse en la figura 2.7, correspondiente a la línea He I $\lambda 4713$ de la mencionada estrella Yakunin et al. 2015.

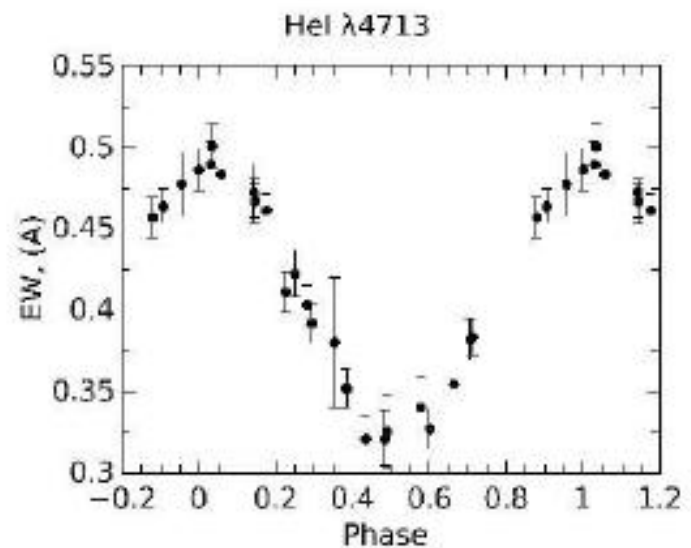

Figura 2.7: Variación del ancho equivalente de He I $\lambda 4713$ con la fase, campo magnético longitudinal con la fase rotación Yakunin et al. 2015.

Se han propuesto varios modelos para explicar las variaciones de los campos magnéticos de las estrellas CP Babcock, 1960a, Ledoux y Renson, 1966), pero la evidencia observacional 
avaló lo que se conoce como Modelo de Rotador Oblicuo, MRO Stibbs 1950. En el MRO se supone que el campo magnético tiene una simetría dipolar simple y que las variaciones del campo magnético se deben a que el eje de la geometría magnética no coincide con el eje de rotación de la estrella. De esta forma, un observador externo, ve distintos aspectos de la geometría magnética a medida que la estrella rota. Así, el MRO explica que las variaciones periódicas del campo son debidas a la rotación de la estrella y que las inversiones de polaridad se deben a la configuración de la geometría particular del campo magnético.

En los años '60 este modelo fue seriamente criticado debido a la aparente existencia de estrellas que poseían variaciones irregulares de sus campos magnéticos Babcock 1960a. Sin embargo, poco tiempo después, se demostró que todas estas estrellas aparentemente irregulares eran en realidad periódicas y presentaban componentes magnéticas multipolares Preston, 1970, Borra y Landstreet, 1980.

Años después, modelos más sofisticados incorporaron componentes multipolares y se consideraron distribuciones no uniformes Deutsch, 1958, Böhm-Vitense 1967, Pyper, 1969. Pero, por otro lado, Landstreet 1970 encontró que podía reproducir la curva de $B_{l}$ si consideraba una geometría dipolar descentrada. Resultados similares se obtuvieron suponiendo una configuración constituida por un dipolo más un cuadrupolo magnético Borra 1980.

En cuanto al ángulo entre los ejes de rotación y magnético, las observaciones indican que, en general, este ángulo es menor que $30^{\circ}$ o mayor que $60^{\circ}$, con predominio de estos últimos, aunque no hay acuerdo entre los autores si la distribución de este ángulo es una distribución uniforme o bimodal.

Un caso especial del MRO, es el rotador simétrico en donde el ángulo de la geometría magnética y el eje de rotación es de $90^{\circ}$. Este modelo fue propuesto por Krause y Oetken 1976) y Oetken 1977, 1979, y utilizaron un campo magnético formado por una componente dipolar y otra cuadrupolar, con contribuciones comparables.

\subsection{Origen del campo magnético}

En cuanto al origen del campo magnético en estrellas masivas, hay dos teorías aceptadas. La que más apoyo concita indica que el campo magnético es un campo magnético fósil Cowling. 1945). La teoría alternativa, postula que el campo magnético se mantiene debido a que recibe una inyección de energía por efecto dínamo Krause y Raedler, 1980, Moffatt, 1978, Parker. 1979.

Se admite que las estrellas de tipos espectrales tardíos o similares al Sol, poseen campos magnéticos generados probablemente por efecto dínamo. Estos campos están presentes en todas las estrellas frías, y están fuertemente asociados con las zonas convectivas de las estre- 
llas y con la velocidad de rotación estelar. Además, presentan variaciones de diversas escalas de tiempo, tal como lo predice la teoría. (Donati y Landstreet, 2009).

Ninguna de estas características se encuentran en las estrellas tempranas de secuencia principal. Los campos magnéticos se encuentran presentes en sólo algunas de estas estrellas y topológicamente hablando son bastantes simples. Tampoco existe una relación con la velocidad de rotación estelar, encontrando que para una misma velocidad de rotación, hay estrellas con campos magnéticos débiles y otras con campos magnéticos relativamente intensos Donati y Landstreet, 2009.

Desde el punto de vista teórico, se ha investigado si estos campos magnéticos podían ser debidos a dínamos operando en el núcleo convectivo, o en una zona radiativa que presenta turbulencia y movimientos por cizallamiento, que son elementos necesarios para que ocurra el efecto dínamo. De hecho, los dínamos de las zonas convectivas de las estrellas enanas M son capaces de formar campos magnéticos intensos con simetría axial Dobler et al. 2006. Browning, 2008, y entonces uno pensaría que podría ocurrir lo mismo en los núcleos convectivos de las estrellas tempranas Charbonneau y MacGregor, 2001, MacGregor y Cassinelli, 2003 Brun et al. 2005. Sin embargo, la dificultad reside en cómo llevar el flujo magnético generado por el dínamo hasta la superficie sin que este deje de operar Dobler et al. 2006. MacDonald y Mullan, 2004, produciendo campos magnéticos simples y estables, como los observados en las estrellas tempranas y, que además, no posean una correlación con la velocidad de rotación de la estrella.

Alternativamente se propuso la idea de un dínamo de cizallamiento operando en las zonas radiativas de las estrella tempranas Spruit, 2002, MacDonald y Mullan, 2004, Mullan y MacDonald, 2005, Braithwaite 2006a. Sin embargo, los campos predichos aún conservan la dependencia con la velocidad de rotación y presentan variaciones temporales, en contradicción con las observaciones. Además, los campos observados son lo suficientemente intensos como para no ser "cortados" por la rotación diferencial y evitar las inestabilidades presentes en el funcionamiento de los dínamos Aurière et al. 2007a.

En este contexto, la idea teórica original de que los campos magnéticos se generaron en algún momento anterior de la historia de la estrella, permaneciendo confinados por la autoinducción magnética, proporciona una explicación más convincente. En esta teoría, conocida como la hipótesis de campos fósiles, los campos magnéticos de las nubes moleculares a partir de las cuales se formaran las estrellas, son transportados y amplificados al colapsar las nubes en protoestrellas. La mayor parte del flujo magnético probablemente se pierde por disipación óhmica o difusión ambipolar durante el proceso de contracción, de forma que sólo algunas protoestrellas son capaces de retener una porción significativa de su flujo magnético. Esto último explicaría por qué sólo unas pocas estrellas tempranas conservan sus campos 
magnéticos Mestel, 1999). En estas estrellas, el campo magnético no puede decaer mucho simplemente por disipación óhmica. Debido a la alta conductividad del plasma, casi completamente ionizado, las escalas de disipación óhmica típicas son del orden de los 10000 millones de años, es decir, tiempos mucho mayores que el tiempo de vida de la estrella en la secuencia principal. De esta forma, cualquier campo magnético altamente comprimido, que haya sobrevivido al proceso de formación estelar, permanecerá casi invariante a lo largo de la vida de la estrella en la secuencia principal. Se espera entonces que tales campos sean estáticos y de estructura relativamente simple, dado su origen, y que no presenten correlaciones con la velocidad de rotación de la estrella. Así, el origen fósil del campo magnético se apoya en la falta de correlación entre la intensidad de los campos magnéticos de las estrellas de masa intermedia y masivas con el período de rotación (entre fracción de día a varios años, ver Kochukhov y Bagnulo, 2006, Wade y MiMeS Collaboration, 2015.

Sin embargo, si los campos fósiles simples son estables es una discusión abierta. Se sabe que los campos poloidales y toroidales son inestables Tayler, 1973 Wright 1973 Braithwaite. 2006b, 2007 y probablemente sea necesario una mezcla de ambos para que se estabilicen. Cálculos numéricos relativamente recientes sugieren que los campos con topologías inicialmente inestables, desarrollan espontáneamente una configuración magnética mixta (poloidal - toroidal) estabilizándose en ese proceso Braithwaite y Spruit, 2004, Braithwaite y Nordlund, 2006.

La existencia aparente de un límite inferior en la detección de las intensidades de los campos magnéticos encontrado en las estrellas Ap Aurière et al. 2007a puede estar relacionado con esta misma inestabilidad. Cuando el campo interno es demasiado débil, se desarrolla una cizalla radial y el campo pasa a tener una configuración predominantemente toroidal e inestable. La única estrella magnética de tipo temprano cuyo campo está potencialmente sujeto a esta inestabilidad es de tipo espectral O, $\zeta$ Ori A Bouret et al. 2008). Esta sería la única de las estrellas tempranas conocidas que poseería un campo magnético que no sería fósil.

Recientemente, Fossati et al. 2016 catalogaron unas 389 estrellas masivas de secuencia principal (de más de $5 M_{\odot}$ ), 61 de ellas poseedoras de campos magnéticos, y encontraron que cuanto mas viejas son las estrellas es menor el número de ellas que presentan campos magnéticos. Ellos atribuyen esta disminución del número de estrellas magnéticas con la edad, al posible decaimiento del campo magnético. También concluyen que los campos magnéticos iniciales de estas estrellas debían ser 3 veces mayores que los actuales.

En las estrellas magnéticas Ap y $\mathrm{Bp}$, las abundancias en la superficie son drásticamente diferentes a las abundancias de las estrellas normales no magnéticas y, obviamente, esto se correlaciona con la presencia de campos magnéticos. Si no hay convección, ni turbulen- 
cia, la difusión atómica hace que los átomos pesados decanten poco a poco, mientras que la aceleración radiativa (transmitida a iones específicos a través de sus líneas espectrales) hace levitar algunas especies atómicas, aumentando su abundancia relativa en la atmósfera y, posiblemente, incluso llegando a escapar de la estrella. Dependiendo de la intensidad relativa de las dos fuerzas (gravedad y radiación) sobre cada especie, y de la orientación del campo magnético, los átomos se acumularán o desaparecerán en distintas regiones de la fotosfera, y esto causaría las anomalías químicas observadas y la falta de homogeneidad de las abundancias superficiales Donati y Landstreet 2009.

La rotación lenta de la mayoría de las estrellas magnéticas de tipo espectral temprano (respecto a las de tipo espectral similar no magnéticas) es la prueba definitiva de que los campos magnéticos tienen un impacto drástico en la formación de las estrellas masivas. Una posibilidad es que los progenitores de las estrellas magnéticas de tipo temprano sufran una pérdida adicional de momento angular a través del viento o, a través de interacciones con sus discos de acreción Stȩpien, 2000. Otra opción, es que las estrellas magnéticas adquieran menos impulso angular de su nube de origen durante el colapso como consecuencia de su flujo magnético inicial.

También se espera que los campos magnéticos afecten significativamente la evolución de las estrellas masivas Maeder y Meynet, 2003, 2004, 2005.

\subsection{Resumen}

En síntesis, las estrellas químicamente peculiares de He, presentan variaciones periódicas en la intensidad y la forma de los perfiles de línea del He, las cuales correlacionan con el período de rotación de la estrella.

Estas estrellas poseen además campos magnéticos, donde la intensidad de sus componentes longitudinales también varía con períodos que coinciden con el período de rotación estelar. Las variaciones en el campo magnético de origen fósil, se explican en general, con el modelo de rotador magnético oblicuo. Estos campos magnéticos afectan la estructura local de la atmósfera, así como también los procesos difusivos dentro de la misma.

En la siguiente sección presentamos las ecuaciones básicas que nos permiten modelar la estructura de una estrella caliente que presenta un campo magnético dipolar. Este modelo será luego aplicado para analizar las variaciones de los perfiles de las líneas de He, teniendo en cuenta la presencia de campos magnéticos, cuyo eje de simetría está inclinado respecto al eje de rotación de la estrella, de forma que consideraremos un modelo de rotador magnético oblicuo. En un tratamiento posterior discutiremos los efectos de la difusión atómica en presencia de campos magnéticos. 


\section{Capítulo 3}

\section{Modelado de fotosferas con campos magnéticos}

Durante muchos años, varios investigadores han trabajado en la búsqueda de una relación entre la distribución heterogénea de elementos químicos que presentan las estrellas CP de la secuencia principal superior y la presencia de campos magnéticos. Estos trabajos se han enfocado a estudiar el modelo de rotador oblicuo (Pyper, 1969, Oetken, 1979) o a estudiar los efectos de la fuerza de Lorentz en la estructura de la fotosfera de las estrellas Ap Carpenter. 1985 LeBlanc et al. 1994, Shulyak et al. 2007, Valyavin et al. 2004. Sin embargo, a excepción del trabajo de Shulyak et al. 2010, muy poco se ha estudiado sobre la influencia de la fuerza de Lorentz en las estrellas Bp. Dedicaremos entonces los próximos capítulos a cubrir fundamentalmente esta área casi inexplorada, enfocándonos principalmente en el efecto de la presión magnética en la atmósfera de una estrella B y su implicancia en la formación de las líneas de He.

Las líneas de He se forman a nivel de la fotosfera de la estrella, supuesta en equilibrio hidrostático, y esta región caracteriza el espectro que observamos. En el caso del tipo de estrellas objeto de nuestro estudio, el transporte de energía en la fotosfera será fundamentalmente radiativo, no habiendo procesos convectivos.

Para tener en cuenta el efecto del campo magnético sobre la atmósfera de una estrella, seguiremos los lineamientos presentados por Valyavin et al. 2004 quienes incluyen dentro de la ecuación de equilibrio hidrostático un término que depende de la fuerza de Lorentz por unidad de volumen $\boldsymbol{f}_{\boldsymbol{L}}$, que está dada por,

$$
\boldsymbol{f}_{\boldsymbol{L}}=\frac{1}{c} \boldsymbol{j} \times \boldsymbol{B},
$$


$\boldsymbol{j}$ es la corriente eléctrica inducida y $\boldsymbol{B}$ es la intensidad del campo magnético. A partir de la expresión de la corriente eléctrica que nos da la ley de Ampère, donde despreciamos las corrientes de desplazamiento, tenemos,

$$
\boldsymbol{j}=\frac{c}{4 \pi} \nabla \times \boldsymbol{B}
$$

Podemos entonces reescribir a la fuerza de Lorentz por unidad de volumen de la siguiente manera,

$$
\boldsymbol{f}_{\boldsymbol{L}}=\frac{1}{4 \pi}(\boldsymbol{\nabla} \times \boldsymbol{B}) \times \boldsymbol{B}
$$

Utilizando la siguiente identidad vectorial,

$$
\frac{1}{2} \boldsymbol{\nabla}(\boldsymbol{B} \cdot \boldsymbol{B})=(\boldsymbol{B} \cdot \boldsymbol{\nabla}) \boldsymbol{B}+\boldsymbol{B} \times(\boldsymbol{\nabla} \times \boldsymbol{B})
$$

obtenemos la siguiente expresión:

$$
\begin{aligned}
(\boldsymbol{\nabla} \times \boldsymbol{B}) \times \boldsymbol{B} & =(\boldsymbol{B} \cdot \boldsymbol{\nabla}) \boldsymbol{B}-\frac{1}{2} \boldsymbol{\nabla}(\boldsymbol{B} \cdot \boldsymbol{B}) \\
& =-\frac{\boldsymbol{\nabla} \boldsymbol{B}^{2}}{2}+(\boldsymbol{B} \cdot \boldsymbol{\nabla}) \boldsymbol{B}
\end{aligned}
$$

Luego, aplicando esta identidad a la ecuación 3.3, obtenemos:

$$
\boldsymbol{f}_{\boldsymbol{L}}=-\frac{\boldsymbol{\nabla} \boldsymbol{B}^{2}}{8 \pi}+\frac{1}{4 \pi}(\boldsymbol{B} \cdot \boldsymbol{\nabla}) \boldsymbol{B}
$$

cuyas componentes pueden escribirse en su forma tensorial (sabiendo que $\boldsymbol{\nabla} \cdot \boldsymbol{B}=0$ y usando la notación de Einstein $[$ de la siguiente manera,

$$
\left(f_{L}\right)_{i}=\frac{\partial}{\partial x_{j}}\left(-\delta_{i j} \frac{B^{2}}{8 \pi}+\frac{B_{i} B_{j}}{4 \pi}\right)
$$

El campo magnético $\boldsymbol{B}$ crea en el plasma una presión magnética isotrópica $\left(B^{2} / 8 \pi\right)$ y una tensión magnética $\left(B_{i} B_{j} / 4 \pi\right)$ dirigida a lo largo de las líneas de fuerza magnética. En la vecindad de las líneas de fuerza de un campo magnético tenemos que la presión magnética trata de expandir el plasma, mientras que la tensión magnética trata de compensar este efecto. La configuración libre de fuerzas se da cuando el gradiente de la presión magnética es completamente compensado por las fuerzas de tensión magnética, es decir, cuando,

\footnotetext{
${ }^{1}$ En la notación de Einstein, cuando aparecen dos índices iguales en el mismo término, significa que se está sumando sobre ese índice.
} 


$$
\frac{\nabla \boldsymbol{B}^{2}}{8 \pi}=\frac{1}{4 \pi}(\boldsymbol{B} \cdot \boldsymbol{\nabla}) \boldsymbol{B}
$$

Cualquier geometría magnética que satisfaga $\boldsymbol{\nabla} \times \boldsymbol{B}=0$ ó $\boldsymbol{\nabla} \times \boldsymbol{B}=h(r) \boldsymbol{B}$ (donde $h(r)$ es una función escalar dependiente de la coordenada radial) corresponde a una configuración libre de fuerzas. Un ejemplo de esta clase de campos es el dipolo magnético, el cual posee simetría axial. Para obtener una fuerza no nula a partir de una configuración de dipolo magnético es necesario distorsionarlo o descentrarlo Parker. 1979.

Está claro que los ejemplos idealizados de geometrías magnéticas altamente simétricas no existen en la naturaleza. Incluso en el caso de una estrella que rota lentamente donde inicialmente tenemos un dipolo magnético, el decaimiento global del campo magnético libre de fuerzas generará corrientes atmosféricas inducidas que darán como resultado la aparición de componentes adicionales del campo magnético que distorsionarán la geometría ideal libre de fuerzas del campo dipolar inicial. La intensidad de las corrientes eléctricas generadas no será grande, pero aún en este caso idealizado, la fuerza de Lorentz debida a la interacción del campo magnético con la corriente eléctrica inducida debería influir sobre las capas atmosféricas superiores.

Por lo antes dicho, si deseamos modelar una situación más realista, típica en estrellas magnéticas, la fuerza de Lorentz no puede ignorarse en los cálculos atmosféricos. Por ejemplo, las estrellas frías con envolturas convectivas tienen campos magnéticos muy complejos concentrados en tubos de flujo magnéticos, con intensidades de unos pocos miles de Gauss. Tales campos están muy lejos de poder considerarse como libres de fuerzas. Las estrellas CP que estudiaremos en este trabajo tienen campos magnéticos superficiales con topologías de multipolaridad baja. Consideraremos entonces que las corrientes eléctricas de la superficie son inducidas por la evolución del campo magnético global y que otras causas que generan corrientes, como por ejemplo la difusión ambipolar, no serán tenidas en cuenta en esta instancia. Este modelo se ajusta bastante bien para estrellas de secuencia principal con temperaturas efectivas entre $8000 K$ y $25000 K$ y bajas velocidades de rotación Landstreet. 1987.

Entonces, para determinar la estructura de presión de la atmósfera, comenzamos considerando que la ecuación de equilibrio hidrostático tiene la siguiente forma,

$$
\nabla P_{t o t}=\rho \boldsymbol{g}+\frac{1}{c} \boldsymbol{j} \times \boldsymbol{B}
$$

donde $P_{\text {tot }}$ es la presión atmosférica total (gas + magnética), $\rho$ es la densidad del gas, $\boldsymbol{g}$ es la aceleración de la gravedad, $\boldsymbol{j}$ indica la corriente eléctrica atmosférica inducida, y $\boldsymbol{B}$ es el vector intensidad del campo magnético. 
En el caso general, la ley de Ohm tiene aproximadamente la siguiente forma Cowling y Lindsay, 1957, Schlüter, 1950,

$$
\boldsymbol{j}=\lambda \boldsymbol{E}_{\|}+\lambda_{1} \boldsymbol{E}_{\perp}-\frac{\lambda_{2}}{B} \boldsymbol{E}_{\perp} \times \boldsymbol{B}
$$

donde $\boldsymbol{E}_{\|}$y $\boldsymbol{E}_{\perp}$ son las componentes del campo eléctrico dirigidas a lo largo y perpendicularmente a las líneas de campo magnético, respectivamente, $\lambda$ es la conductividad eléctrica en ausencia de campo magnético, $\lambda_{1}$ es la conductividad a lo largo de las líneas de campo magnético y $\lambda_{2}$ es la conductividad debida al efecto Hall. A partir de las propiedades de un plasma autogravitante Cowling y Lindsay, 1957 Schlüter 1950, a lo largo de las líneas de campo magnético, la conductividad $\lambda_{1}$ es pequeña comparada con la conductividad $\lambda$ :

$$
\lambda_{1}=\frac{\lambda}{1+(\omega \tau)^{2}}
$$

donde $\omega$ es la frecuencia ciclotrón y $\tau$ es el tiempo libre medio de las partículas conductoras. El tercer término de la ecuación (3.8) representa la corriente por efecto Hall. Esta corriente aparece como consecuencia de la deriva de las partículas cargadas moviéndose en espiral en el campo magnético. La dirección de la corriente por efecto Hall está definida por el producto vectorial $\boldsymbol{E}_{\perp} \times \boldsymbol{B}$. De esta forma, en el plano ecuatorial de un dipolo magnético, la corriente por efecto Hall tendrá dirección radial.

La conductividad eléctrica de esta corriente estará dada por:

$$
\lambda_{2}=\frac{\lambda \omega \tau}{1+(\omega \tau)^{2}}
$$

A partir de las ecuaciones (3.9) y 3.10 se puede ver que la corriente por efecto Hall es insignificante en las capas atmosféricas más profundas donde $\omega \tau<<1$. Debemos notar que en estas capas, la conductividad eléctrica a través de las líneas de campo magnético es cercana al caso no magnético.

Definiremos entonces, una condición formal para cuando la corriente por efecto Hall no puede ser despreciada. Esto ocurre, en las capas de la atmósfera, donde

$$
\omega \tau=1
$$

y se corresponde al caso donde la conductividad eléctrica a través de las líneas de campo está reducida con respecto al caso no magnético en un factor 2. En otro sentido, estas capas también definen los puntos de la atmósfera (donde $\lambda_{2} \approx \lambda_{1} \approx \lambda / 2$ ) por sobre las cuales la corriente por efecto Hall aumenta rápidamente. De esta forma, la expresión 3.11 actúa 
como un criterio para determinar donde despreciar o no las corrientes por efecto Hall.

En general, la presencia de un campo magnético conduce a una distorsión de una distribución simétrica sencilla de una corriente atmosférica. Por lo que, en nuestro caso, la suposición de que la corriente eléctrica puede ser descripta sólo a través de la componente azimutal se torna cuestionable. Además, la presencia de componentes de deriva de la corriente eléctrica atmosférica puede llevar a generar campos eléctricos inducidos adicionales y a desarrollar movimientos de deriva de plasma globales similares a la circulación meridional Landstreet. 1987). Estos efectos sólo pueden estudiarse con cálculos magnetohidrodinámicos 3D autoconsistentes dependientes del tiempo, que están fuera del alcance de este trabajo.

\subsection{Suposiciones para el modelado de los campos mag- néticos}

Consideraremos que la superficie del campo magnético tiene una configuración cercana a la de un campo multipolar de baja simetría axial y utilizaremos un campo dipolar que fácilmente puede extenderse a otra configuración poloidal con simetría axial. Estas consideraciones son similares a las utilizadas por Madej 1983. La topología del campo magnético puede ser distinta en el interior estelar pero no es de interés para nuestro estudio, ya que las líneas no se forman allí.

Para calcular la estructura hidrostática teniendo en cuenta las fuerzas magnéticas deben hacerse varias suposiciones.

Primero, supondremos que el espesor de la atmósfera es despreciable frente al radio de la estrella, y para poder modelarla podremos descartar los efectos de curvatura, por lo que la supondremos formada por capas plano paralelas. Segundo, supondremos que las capas atmosféricas magnetizadas están en equilibrio hidrostático. Tercero, consideraremos que el campo magnético de la superficie estelar está dominado por la componente dipolar libre de fuerzas $(\boldsymbol{\nabla} \times \boldsymbol{B}=0)$ en todas las capas de la atmósfera.

Bajo estas suposiciones las expresiones para las componentes del dipolo magnético en coordenadas esféricas en el sistema de referencia del dipolo están dadas por:

$$
\begin{aligned}
B_{r} & =\frac{R_{\star}^{3} B_{p}}{r^{3}} \cos \theta, \\
B_{\theta} & =\frac{R_{\star}^{3} B_{p}}{2 r^{3}} \operatorname{sen} \theta, \\
B_{\varphi} & =0,
\end{aligned}
$$


donde $B_{p}$ es la intensidad del campo magnético polar, $\theta$ y $\varphi$ son los ángulos de latitud y azimut magnéticos, respectivamente, $r$ es la coordenada radial y $R_{\star}$ es el radio de la estrella. Como mencionamos anteriormente, la distorsión del campo dipolar es creada por la evolución temporal del campo y puede incluirse por medio de la corriente eléctrica azimutal inducida $\boldsymbol{j}$. Tal distorsión es equivalente a sumar al campo dipolar un campo magnético adicional, $\boldsymbol{B}_{\boldsymbol{a d d}}$, el cual se obtiene a partir de la ecuación de Maxwell,

$$
\nabla \times \boldsymbol{B}_{a d d}=\frac{4 \pi \boldsymbol{j}}{c}
$$

Por último, el apartamiento de la simetría axial de las corrientes eléctricas inducidas causado por los efectos dinámicos relacionados a la rotación estelar y a la posible presencia de corrientes por efecto Hall será despreciado.

Debido a la simetría axial que tiene el campo dipolar, su evolución temporal crea una corriente eléctrica que sólo posee componente azimutal: $j_{\varphi}=j_{e q} \operatorname{sen} \theta$, donde $j_{e q}$ es la corriente atmosférica en el plano ecuatorial del dipolo. En este caso, la componente radial escalar de la ecuación (3.7) tiene la siguiente forma:

$$
\frac{d P_{t o t}}{d r}=-\rho g \pm \frac{j_{e q}}{c} B_{\theta} \operatorname{sen} \theta
$$

donde el signo + corresponde al caso de la fuerza de Lorentz dirigida hacia adentro, mientras que el signo - indica que la fuerza estará dirigida hacia el exterior. Esta fórmula puede extenderse a cualquier campo poloidal con simetría axial teniendo en cuenta que, generalmente, la distribución superficial de corrientes inducidas puede expresarse en términos de los polinomios asociados de Legendre $j_{\varphi} \sim P_{n}^{l}(\cos \theta)$ Wrubel, 1952, donde $n=1$ para el dipolo, $n=2$ para el cuadrupolo, etc.

Para un campo dipolar, la corriente eléctrica inducida en el plano ecuatorial, $j_{e q}$, puede ser descripta por la función $\Phi$, que depende de tres cantidades,

$$
j_{e q}=\Phi\left(\lambda, E_{e q}, B_{e q}\right)
$$

donde $\lambda$ es la conductividad eléctrica, $E_{e q}$ es el campo eléctrico ecuatorial inducido por la evolución temporal del campo magnético en la superficie, y $B_{e q}$ es la intensidad del campo magnético en el ecuador.

Notemos que las desviaciones respecto de una situación idealizada tienen lugar en las capas superiores de la atmósfera, donde la frecuencia de ciclotrón de las partículas cargadas es mayor que la frecuencia de colisión efectiva. En los siguientes párrafos realizaremos una 
evaluación cuantitativa para caracterizar la posible desviación de un campo magnético en nuestro modelo a partir de la configuración de equilibrio.

Considerando las hipótesis propuestas, aplicaremos este modelo a estrellas de tipo espectral B de secuencia principal, por lo que podemos aproximar la ley de Ohm de la siguiente manera,

$$
\boldsymbol{j}=\lambda_{1} \boldsymbol{E}_{\perp}
$$

Notemos que, debido a nuestras suposiciones, la componente de la corriente eléctrica inducida, $\boldsymbol{E}_{\|}$, paralela al campo magnético se encuentra ausente en el modelo. De esta forma, la expresión escalar definitiva para la corriente eléctrica inducida tiene la siguiente forma,

$$
j_{\varphi}=\frac{E_{e q} \lambda}{1+(\omega \tau)^{2}} \operatorname{sen} \theta
$$

Aplicando esto de modo general para atmósferas con partículas cargadas de cualquier tipo, obtenemos nuestra solución para la componente radial de la fuerza de Lorentz a través de la gravedad efectiva. De esta forma la ecuación de equilibrio hidrostático queda expresada,

$$
\frac{d P_{t o t}}{d r}=-\rho g_{e f f}
$$

donde la gravedad efectiva $g_{\text {eff }}$ está dada por la expresión,

$$
g_{e f f}=g \pm \sum_{i} \frac{\lambda_{i} \operatorname{sen} \theta}{c \rho\left(1+\left(\omega_{i} \tau_{i}\right)^{2}\right)} E_{e q} B_{\theta}
$$

donde $g$ es la gravedad superficial de un modelo sin campo magnético, $c$ es la velocidad de la luz, y $\rho$ es la densidad de masa. $\lambda_{i}$ es la conductividad eléctrica de la partícula $i, \omega_{i}$ es su frecuencia ciclotrón y $\tau_{i}$ es el tiempo del camino libre medio. El índice $i$ de la sumatoria indica que la suma se realiza sobre las diferentes clase de partículas cargadas presentes en la atmósfera. $\theta$ es el ángulo de latitud, $E_{e q}$ es el campo eléctrico en el ecuador y $B_{\theta}$ es la componente latitudinal del campo magnético definida en la ecuación 3.12b.

\subsection{Cálculo de las conductividades eléctricas $\lambda_{i}$}

Para poder calcular la gravedad efectiva, $g_{\text {eff }}$, es necesario conocer las conductividades eléctricas $\lambda_{i}$ y los tiempos del camino libre medio $\tau_{i}$. Para un plasma de baja densidad en ausencia de un campo magnético podemos usar las siguientes expresiones: 


$$
\tau_{i}=\frac{l_{i}}{\left\langle v_{i}\right\rangle}
$$

donde $l_{i}$ es el camino libre medio, el índice $i$ indica el tipo de partícula y $\left\langle v_{i}\right\rangle$ es la velocidad térmica media dada por:

$$
\left\langle v_{i}\right\rangle=\sqrt{\frac{3 k_{B} T}{m_{i}}}
$$

siendo $k_{B}$ la constante de Boltzmann, $T$ la temperatura y $m_{i}$ la masa de la partícula. En el caso general,

$$
l_{i}^{-1}=\sum_{k} l_{i k}^{-1}
$$

donde $l_{i k}$ es el camino libre medio de las partículas de tipo $i$ en interacción con las partículas de tipo $k$. Los métodos para calcular los $l_{i k}$ son derivados de la teoría cinética de los gases y nosotros utilizamos la solución dada por Pikelner 1966,

$$
\begin{aligned}
l_{e e} & =\frac{\left(k_{B} T\right)^{2}}{n_{e} e^{4} L_{e e}}, \\
l_{i k} & =\frac{\left(k_{B} T\right)^{2}}{n_{k} e^{4} L_{i k} Z_{i}^{2} Z_{k}^{2}}, \\
l_{e k} & =\frac{\left(k_{B} T\right)^{2}}{n_{k} e^{4} L_{e k} Z_{k}^{2}},
\end{aligned}
$$

donde el subíndice $e$ hace referencia al gas de electrones y $Z_{i}$ es el grado de ionización de la partícula $i$. En el caso de los electrones, el grado de ionización es igual a 1.

Las cantidades auxiliares $L_{i k}$ están dadas por:

$$
L_{i k}=2 \ln \left[\frac{3\left(k_{B} T\right)^{3 / 2}}{2 \pi^{1 / 2} n_{k}^{1 / 2} e^{3} Z_{i} Z_{k}}\right]
$$

El camino libre medio de las partículas de tipo $i$ en interacción con los átomos neutros de tipo a estará dado por:

$$
l_{i a}=\frac{1}{\sigma_{a i} n_{a}}
$$

donde $n_{a}$ es la densidad del número de partículas del átomo de tipo $a$ y $\sigma_{a i}$ es la sección 
eficaz del átomo neutro de tipo $a$ ante un ión de tipo $i$ incidente, y viene dada por:

$$
\sigma_{a i}=\frac{h^{2}\left(m_{i}+m_{a}\right)}{m_{i} m_{a} v_{i}^{2}}
$$

y $v_{i}$ es la velocidad de la partícula incidente. Por último, la conductividad eléctrica de los iones del tipo $i$ estará dada por:

$$
\lambda_{i}=\frac{n_{i} e_{i}^{2} \tau_{i}}{m_{i}}
$$

Una vez determinadas las conductividades podemos calcular la gravedad efectiva y proceder a resolver numéricamente la ecuación 3.18 como se detalla en el próximo capítulo. 


\section{Capítulo 4}

\section{Aplicación a las estrellas B magnéticas}

Para confeccionar la estructura de una fotosfera no gris, en equilibrio radiativo y equilibrio termodinámico local, bajo la acción de un campo magnético, es necesario resolver la ecuación diferencial 3.18. Para ello, adaptamos un código, confeccionado por René Rorhmann, que resuelve un modelo de atmósfera de capas plano paralelas en ausencia de campos magnéticos Rohrmann 2001) y Rohrmann et al. 2002 al que incorporamos los efectos de la presión magnética, que se representa a través del término de la gravedad efectiva dada por la ecuación 3.19 empleando las conductividades eléctricas descriptas en la sección \$3.2.

Una descripción del contenido de estos códigos se presenta en el apéndice de este trabajo de Tesis. En la sección A.1 se describe brevemente el código desarrollado originalmente por Rohrmann, y las correspondientes adaptaciones se presentan en la sección A.2.

El acoplamiento entre ambos códigos se realizó empleando un procedimiento iterativo como se describe en la sección A.3.

Los resultados del modelo de atmósfera con campo magnético se aplicaron a estrellas de tipo espectral B con temperaturas entre $11000 \mathrm{~K}$ y $25000 \mathrm{~K}$.

Para verificar el buen funcionamiento de nuestro código, comparamos nuestros resultados con el modelo publicado por Valyavin et al. 2004, quien utilizó una adaptación del código de Kurucz para incluir el cálculo de la gravedad efectiva. Esta comparación se discute en la próxima sección.

\subsection{Comparación de los modelos con trabajos previos}

En las figuras 4.1 y 4.2 se puede ver el comportamiento del cociente entre la gravedad efectiva y la gravedad del modelo en ausencia de campo magnético en función de la profundidad óptica 
(en escala logarítmica), correspondiente a modelos con la fuerza de Lorentz dirigida hacia el exterior e interior de la estrella, respectivamente.
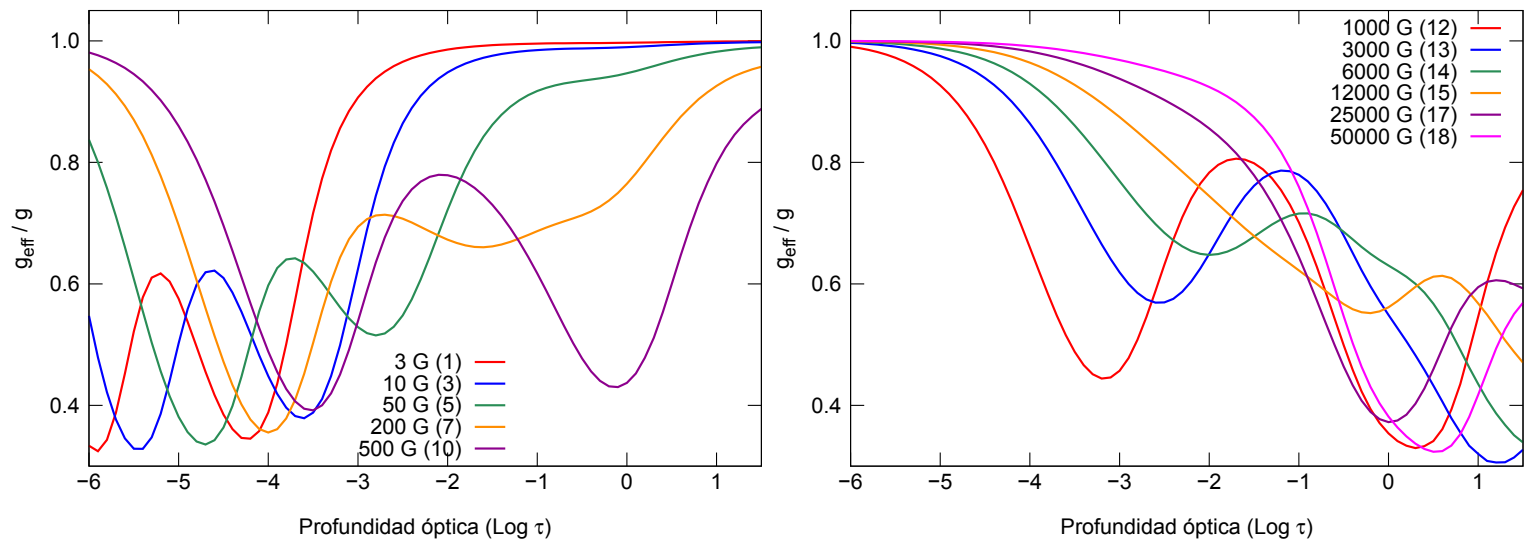

Figura 4.1: Aquí mostramos nuestros resultados de la variación de la gravedad efectiva aplicados a un modelo de una estrella de $11000 \mathrm{~K}$, similar al utilizado por Valyavin et al. 2004, y para las mismas intensidades de campo magnético que vemos en la figura 4.3. con la fuerza de Lorentz dirigida hacia el exterior de la estrella.
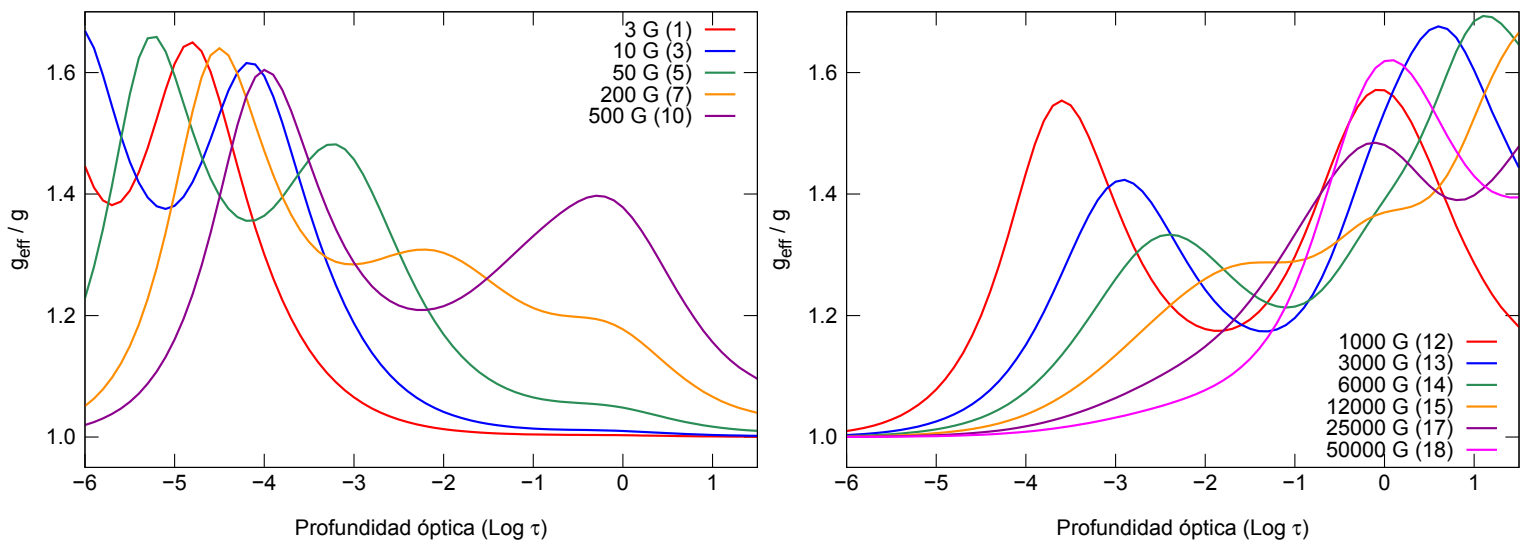

Figura 4.2: Al igual que en la figura anterior (figura 4.1, aquí mostramos nuestros resultados para la gravedad efectiva para los mismos modelos que los calculados por Valyavin et al. 2004, pero con la fuerza de Lorentz dirigida hacia el interior.

En los gráficos de la izquierda se muestran nuestros modelos para intensidades del campo magnético de $3 G(1), 10 G(3), 50 G(5), 200 G(7)$ y $500 G(10)$, mientras que en los de la derecha se corresponden a intensidades de $1000 G(12), 3000 G(13), 6000 G(14), 12000 G$ (15), $25000 G(17)$ y $50000 G(18)$. Entre paréntesis se indica el número del modelo correspondiente con el trabajo de Valyavin et al. 2004.

Se puede apreciar que el comportamiento de la gravedad efectiva es el mismo que se observa en el trabajo de Valyavin et al. 2004, con los máximos y mínimos ubicados a profundidades ópticas similares (como se puede comparar en la figura 4.3).

En nuestro caso el rango de la profundidad óptica del continuo (opacidad de Rosseland) $(\log \tau)$ está definido entre -6 y 1.5, a diferencia de los modelos de Valyavin et al. 2004, en 
donde $\log \tau$ varía entre -7 y 2 (ver figura 4.3).

La diferencia entre los valores que toma la gravedad efectiva en nuestros modelos y en los de Valyavin et al. 2004 se atribuye a los distintos códigos que se utilizaron para determinar la estructura de la atmósfera. En nuestro caso usamos el código desarrollado por Rohrmann (2001) y Rohrmann et al. 2002), que modela una atmósfera constituida básicamente de hidrógeno y helio, mientras que Valyavin et al. 2004 utilizaron los modelos de Kurucz.
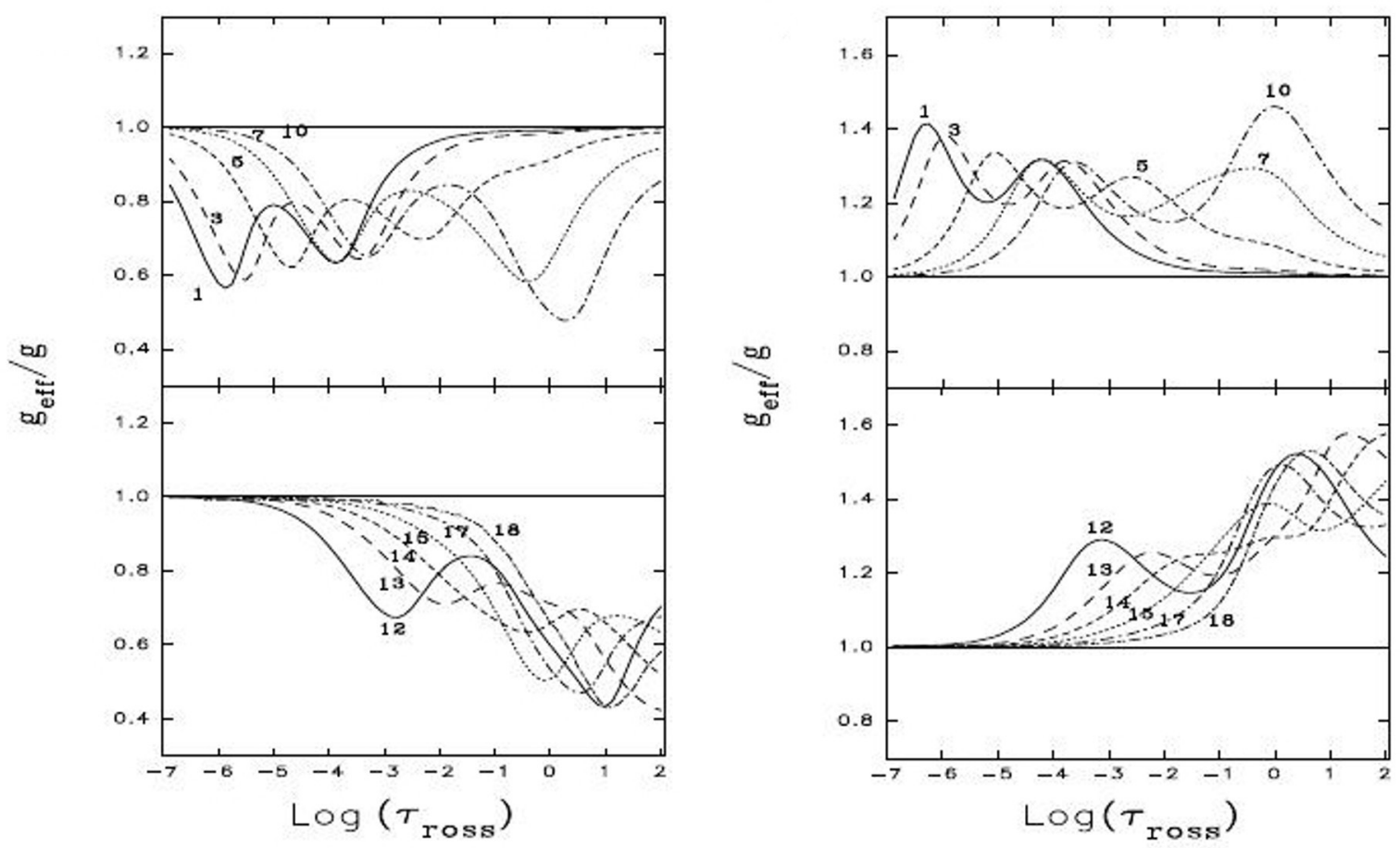

Figura 4.3: Aquí mostramos la figura 2 del trabajo de Valyavin et al. 2004. En ambos gráficos se muestra la gravedad efectiva en función de la profundidad óptica para modelos de una estrella de $11000 \mathrm{~K}$ y distintas intensidades del campos magnéticos: $3 G(1), 10 G(3), 50 G(5), 200 G(7), 500 G(10), 1000 G(12), 3000 G$ (13), $6000 G(14), 12000 G(15), 25000 G$ (17) y $50000 G$ (18). El dibujo de la izquierda corresponde a modelos con la fuerza de Lorentz dirigida hacia el exterior de la estrella, mientras que el de la derecha corresponde a la fuerza de Lorentz dirigida hacia el interior.

Se puede ver que la gravedad efectiva presenta dos máximos relativos, o mínimos, dependiendo de la dirección de la fuerza de Lorentz, entrante o saliente. La profundidad óptica en la cual ocurren estos máximos y mínimos depende de la intensidad del campo magnético. Por otro lado, notamos que los valores de la gravedad efectiva en cada uno de los máximos (o mínimos) son bastante distintos.

En la figura 4.4 mostramos los resultados que hemos obtenido para las conductividades. En estas figuras vemos las conductividades calculadas para un modelo sin campo magnético (negro) y dos modelos con campos magnéticos de $50 G$ y $500 G$ (rojo y azul, respectivamente). Las líneas continuas corresponden a la marcha de la conductividad de los electrones mientras que las líneas con símbolos corresponden a la del hidrógeno ionizado. Nuevamente, la imagen de la izquierda fue obtenida para un modelo donde la fuerza de Lorentz está dirigida hacia el 
exterior de la estrella, mientras que la de la derecha corresponde con un modelo con la fuerza dirigida hacia el interior. Si comparamos estos resultados con los obtenidos por Valyavin et al. 2004) (ver figura 4.5, vemos que ambos presentan un muy buen acuerdo.
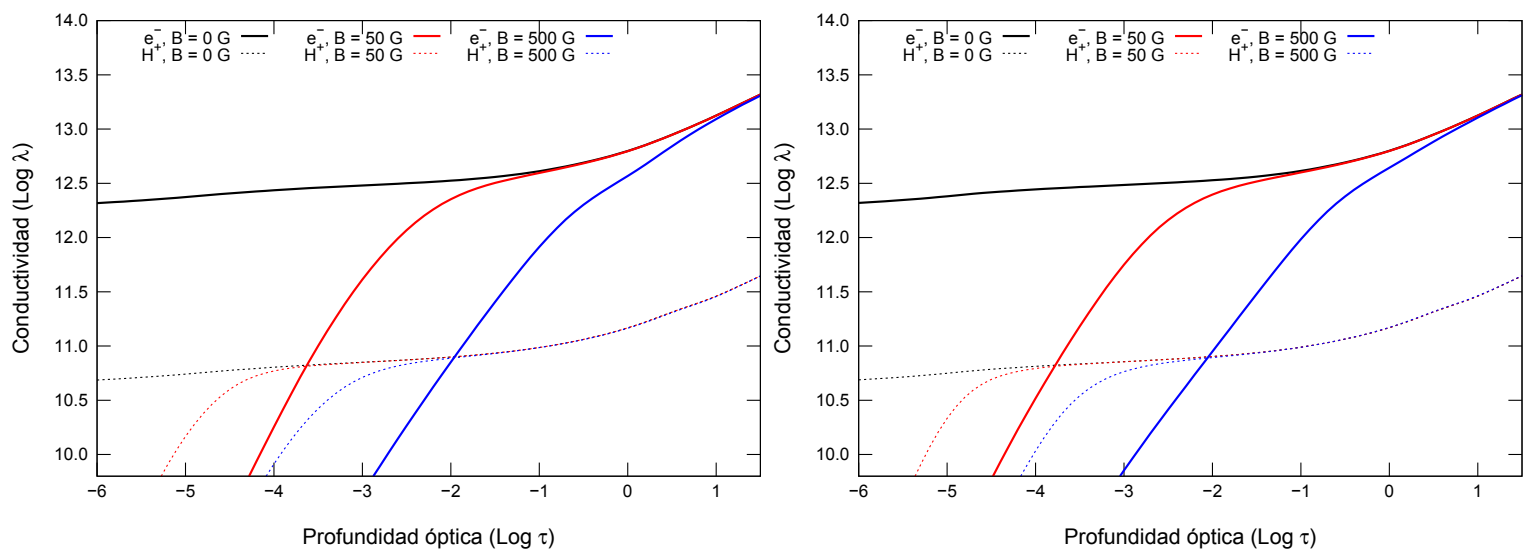

Figura 4.4: Marcha de las conductividades en una estrella de $11000 \mathrm{~K}$. En ambos gráficos se muestra el logaritmo de la conductividad en función del logaritmo de la profundidad óptica. Las líneas continuas corresponden a los electrones y las líneas de trazos corresponden al hidrógeno ionizado. Las líneas negras corresponden a modelos sin campo magnético, las rojas a una intensidad del campo magnético de $50 G(5)$ y las azules a modelos de $500 G(10)$. El dibujo de la izquierda corresponde a modelos con la fuerza de Lorentz dirigida hacia el exterior de la estrella, mientras que el de la derecha corresponde a un modelo con la fuerza de Lorentz dirigida hacia el interior.
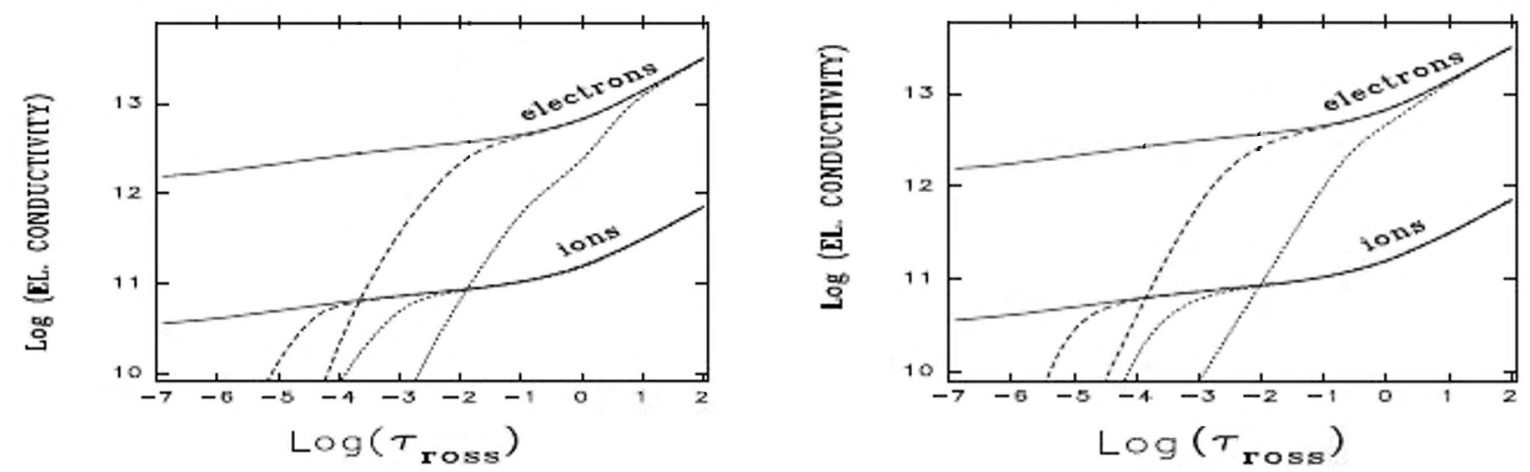

Figura 4.5: Aquí mostramos la figura 3 del trabajo de Valyavin et al. 2004. En ambos gráficos se muestra la conductividad de electrones e iones en función de la profundidad óptica para modelos de una estrella de $11000 \mathrm{~K}$. La línea llena corresponde a modelos sin campo magnético, la entrecortada a un modelo con la intensidad del campo magnético de $50 G$ (modelo 5) y la punteada a un modelo de $500 G$ (modelo 10). El dibujo de la izquierda corresponde a modelos con la fuerza de Lorentz dirigida hacia el exterior de la estrella, mientras que el de la derecha corresponde a un modelo con la fuerza de Lorentz dirigida hacia el interior.

Vemos que la conductividad, tanto de los electrones como la de los iones, decrece suavemente hacia el exterior de la estrella, mientras que en los modelos con campos magnéticos, en este caso $50 G$ y $500 G$, las conductividades decrecen abruptamente a partir de una dada profundidad óptica, la cual depende de la intensidad del campo magnético. Por otro lado, vemos que casi no hay diferencia en los resultados con la dirección de la fuerza de Lorentz, ya que la conductividad no es dependiente de la misma. Las pequeñas diferencias que hay 
entre los modelos con distintas direcciones de la fuerza de Lorentz se deben a las distintas densidades de partículas en cada capa de la atmósfera que sí dependen de la dirección de la fuerza de Lorentz.

\subsection{Comportamiento de la gravedad efectiva con el tipo espectral}

En esta sección analizaremos cómo es el comportamiento de la gravedad efectiva para distintos tipos espectrales, o temperaturas efectivas.

A continuación mostramos los modelos calculados para tres intensidades del campo magnético: 100 G, 1000 G y 3000 G. En todos los casos la fuerza de Lorentz está dirigida hacia el interior de la estrella. Para cada intensidad de campo magnético, mostramos ocho modelos correspondientes a distintas temperaturas efectivas características de distintos tipos espectrales: $23000 \mathrm{~K}$ (B1), $21000 \mathrm{~K}$ (B2), $17600 \mathrm{~K}$ (B3), $15200 \mathrm{~K}$ (B5), $14300 \mathrm{~K}$ (B6), 13500 K (B7), $12300 \mathrm{~K}(\mathrm{~B} 8)$ y $11400 \mathrm{~K}$ (B9).

Como puede apreciarse en los gráficos de la figura 4.6. en donde cada uno representa una intensidad de campo magnético distinta, en líneas generales, la gravedad efectiva decrece al disminuir la temperatura.

En el caso de un campo magnético de 100 Gauss de intensidad (gráfico superior izquierdo de la figura 4.6, vemos que el máximo local más exterior del cociente $g_{\text {eff }} / g$ disminuye levemente al disminuir la temperatura, mientras que el máximo local más interior disminuye mucho más. Así vemos que, en el caso de modelos de temperaturas más altas, los dos máximos locales toman valores similares, mientras que para estrellas más tardías, el comportamiento es similar al obtenido por Valyavin et al. 2004. Además, observamos que ambos máximos locales se van desplazando más hacia afuera de la atmósfera como consecuencia de la estructura de densidad de la atmósfera.

El caso de un campo magnético de 1000 Gauss es mostrado en el gráfico superior derecho de la figura 4.6. Aquí vemos que para estrellas más frías, el valor de los máximos relativos disminuye, pero ambos lo hacen de la misma manera, siendo para cada temperatura prácticamente iguales. También se observa que, al bajar la temperatura, el máximo exterior se mueve más hacia el exterior, mientras que el interior se desplaza hacia el interior. Es decir, los máximos relativos están más separados cuando más baja es la temperatura.

El tercer caso que mostramos aquí, a modo de ejemplo, es el comportamiento de la gravedad efectiva para un campo de 3000 Gauss de intensidad (gráfico inferior de la figura 4.6). Aquí ocurre el caso contrario a lo que teníamos cuando la intensidad de campo magnético era de 100 Gauss. Se observa que el máximo local interior disminuye poco al considerar modelos 
de menor temperatura, mientras que el máximo relativo ubicado en la parte externa de la atmósfera decrece mucho más que el primero para estrellas con temperaturas más bajas. Este comportamiento coincide con lo que hemos visto al comparar con los modelos obtenidos por Valyavin et al. 2004.
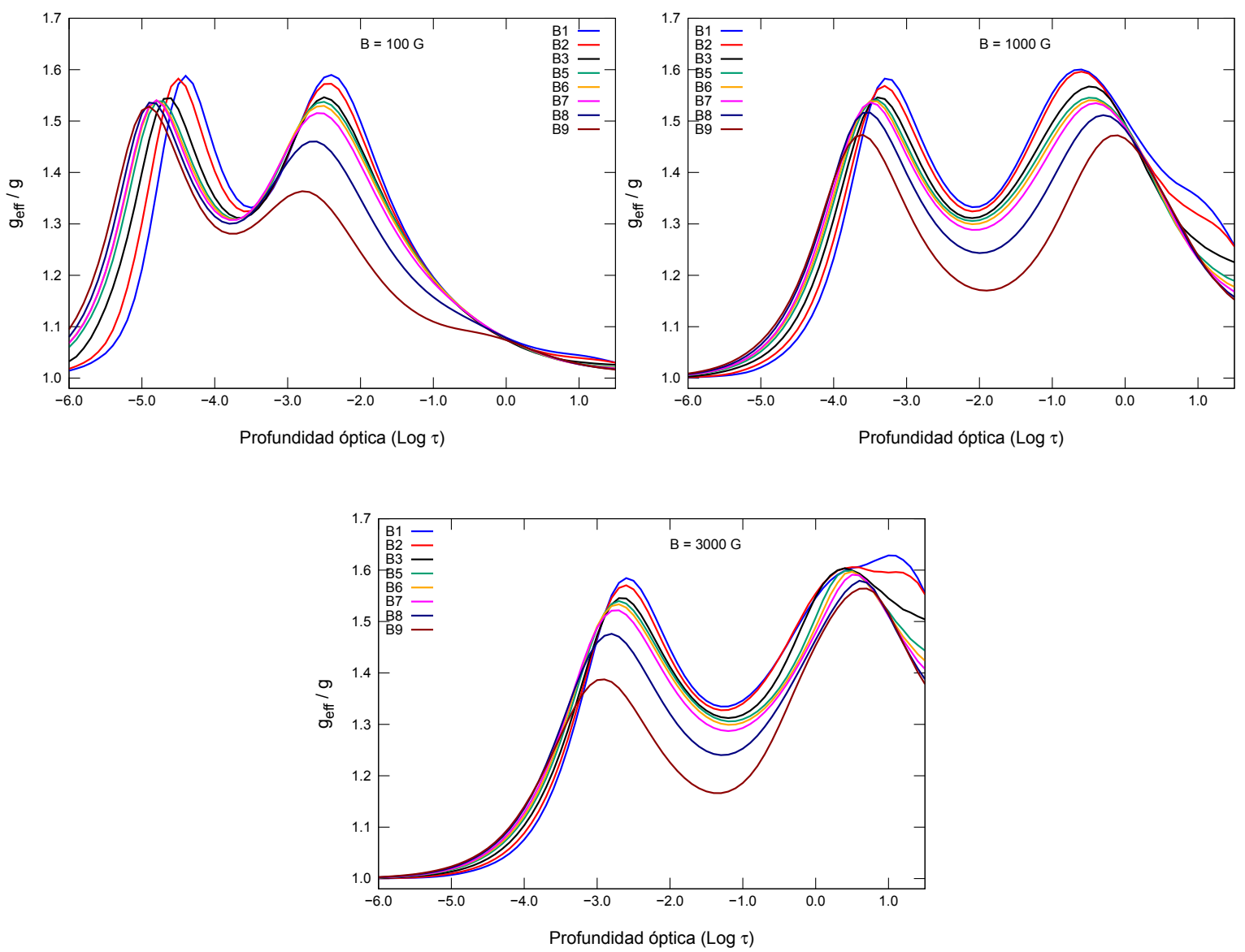

Figura 4.6: Variación del cociente $g_{e f f} / g$ en función del logaritmo de la profundidad óptica, para modelos correspondientes a distintos tipos espectrales. La fuerza de Lorentz está dirigida hacia el interior de la estrella. El gráfico izquierdo superior corresponde a una intensidad del campo magnético de 100 Gauss y el superior derecho corresponde a 1000 Gauss, mientras que el inferior corresponde a un campo de 3000 Gauss.

También se observa, como en el caso de los modelos con intensidades magnéticas de 1000 Gauss, que los máximos relativos se van separando. Para los tipos espectrales más tempranos se ve que el máximo relativo más interno se ve ensanchado.

En el caso de que la fuerza de Lorentz esté dirigida hacia el exterior, el comportamiento es igual al aquí descripto con la salvedad de que en lugar de máximos relativos, tendremos mínimos relativos.

En la subsección siguiente describiremos resultados referentes a la influencia del campo magnético para un subtipo particular a fin de hacer una descripción detallada sobre la estructura de la atmósfera resultante. 


\subsubsection{Descripción de un modelo típico}

Debido a que nuestro trabajo está centrado en estrellas peculiares en He, analizaremos un modelo prototipo correspondiente a una estrella B2 de secuencia principal. Los parámetros que caracterizan a esta estrella son: una temperatura superficial de $22000 \mathrm{~K}$, una gravedad superficial de 4.0 dex $(\log g)$ y un radio de $5.6 R_{\odot}$. Para nuestros modelos hemos considerado una abundancia de He en fracción de masa igual a la solar.

Aquí mostramos nuestros resultados en lo referente a las variaciones de la gravedad efectiva para distintos valores del campo magnético y con la fuerza de Lorentz en ambas direcciones. Asimismo, todos los modelos corresponden a un ángulo latitudinal magnético de $90^{\circ}$ (ecuador magnético), ya que para ese ángulo las variaciones calculadas son máximas. En la sección (4.4 mostraremos como varía la gravedad efectiva con la latitud magnética para un campo magnético dado.

En los conjuntos de figuras 4.7 y 4.8 mostramos las dependencia del cociente $g_{\text {eff }} / g$. Hemos calculado los modelos con intensidades de campos magnéticos de 3, 5, 10, 50, 100, 200, 300, 400, 500, 750, 1000, 2000, 3000, 6000, 12 000, 18 000, 25000 y 50000 Gauss, pero para una mejor visualización, en cada gráfico mostramos el cociente $g_{e f f} / g$ de sólo tres modelos con distinta intensidad de campo magnético.

La figura 4.7 corresponde a un conjunto de modelos con la fuerza de Lorentz dirigida hacia el interior de la estrella, mientras que el conjunto de la figura 4.8 se corresponde a modelos con la fuerza de Lorentz hacia el exterior.

Si nos detenemos a observar los casos en que la fuerza de Lorentz está dirigida hacia el interior, vemos que el comportamiento del cociente $g_{\text {eff }} / g$ muestra dos picos (máximos locales) de altura casi igual. Aquí, sólo cuando los campos magnéticos son muy intensos (por encima de los 3000 Gauss) se observa que el pico correspondiente a profundidades ópticas más internas es levemente mayor que el correspondiente a profundidades ópticas más externas. Además, se ve que cuando el campo magnético es más intenso, las curvas se desplazan hacia profundidades ópticas más internas. Este comportamiento es igual al detectado en los modelos calculados por Valyavin et al. 2004, aunque en estos casos el desplazamiento hacia el interior es mayor.

Un comportamiento similar ocurre cuando la fuerza de Lorentz está dirigida hacia el exterior, en donde encontramos valles (mínimos locales) en el comportamiento del cociente $g_{\text {eff }} / g$ en función con la profundidad óptica.

Claramente se observa el corrimiento de los máximos (mínimos) locales del cociente $g_{\text {eff }} / g$ que se va moviendo hacia el interior de la estrella, de forma tal que para campos mayores a 18000 Gauss, sólo tenemos un máximo (mínimo) dentro del rango de profundidades ópticas 

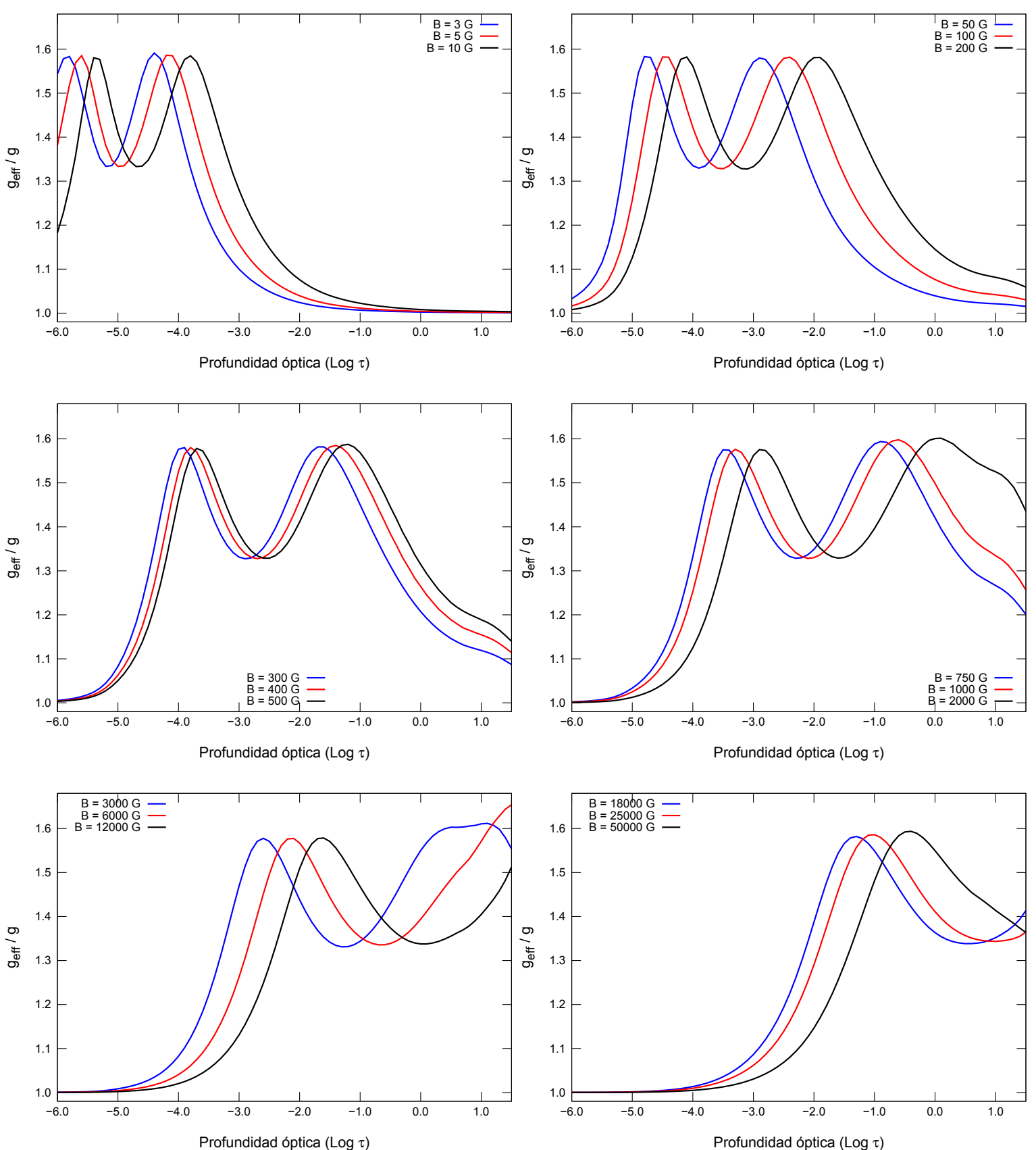

Figura 4.7: Variación de la gravedad efectiva con la profundidad óptica para un modelo de una estrella de $22000 K$ y distintas intensidades de campo magnético. La fuerza de Lorentz está dirigida hacia el interior de la estrella.

para las que calculamos nuestros modelos.

Para estos mismos modelos (ver figura 4.9), mostramos cómo varían las conductividades eléctricas en función de la profundidad óptica al cambiar la intensidad del campo magnético. En el panel de arriba, se muestra la conductividad eléctrica de los electrones, en el panel central, las correspondientes conductividades del hidrógeno ionizado (H II), y en el de abajo, se exponen las conductividades del He una vez ionizado (He II). Para una visualización más 

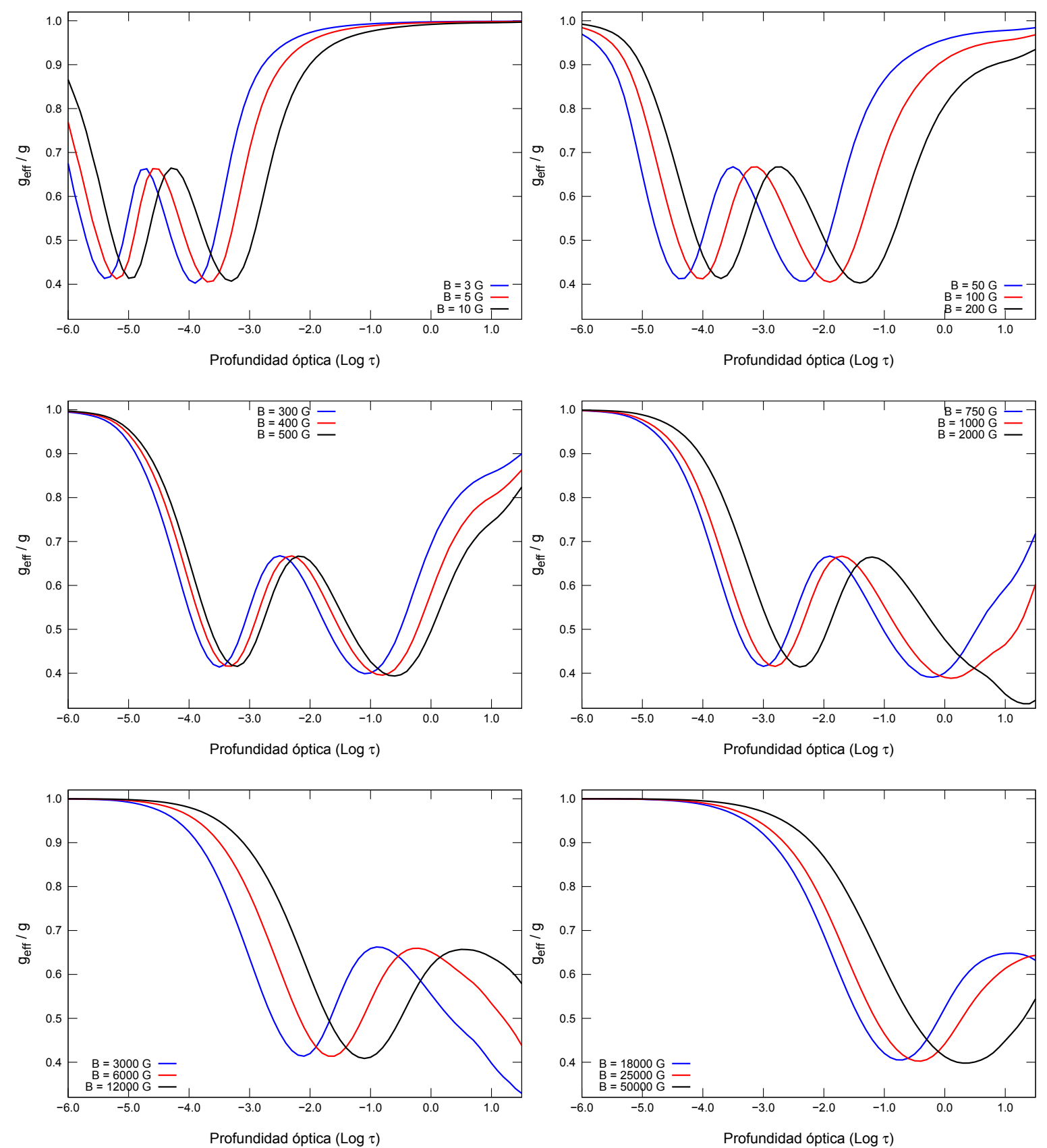

Figura 4.8: Variación de la gravedad efectiva en función de la profundidad óptica para un modelo de una estrella de $22000 K$ y distintas intensidades de campo magnético. La fuerza de Lorentz está dirigida hacia el exterior de la estrella.

clara, no hemos incluido todos los modelos calculados. Por otro lado, las conductividades eléctricas no dependen de la dirección de la fuerza de Lorentz.

En todos los gráficos se muestra a modo de referencia, la correspondiente conductividad eléctrica en ausencia de un campo magnético (en color negro) para poder comparar los modelos con los casos en donde se incluye la presencia de campos magnéticos. Las conductividades eléctricas en ausencia de campos magnéticos decrecen suavemente con la profundidad 

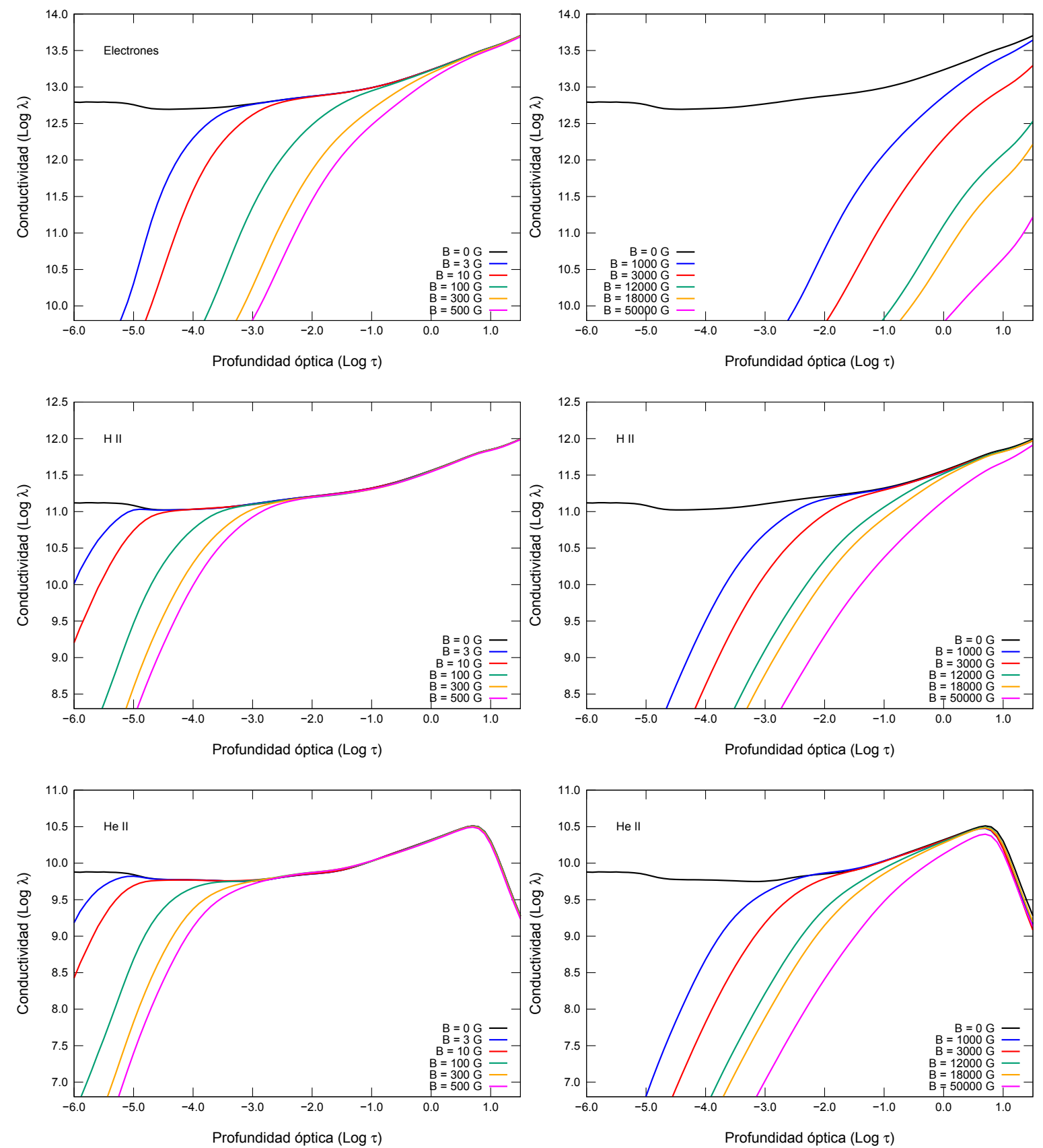

Figura 4.9: En el panel de arriba se muestra la conductividad de los electrones en función de la profundidad óptica para un modelo de una estrella de $22000 \mathrm{~K}$ y distintas intensidades de campo magnético. En ambos casos se muestra como referencia la conductividad de los electrones en ausencia de campo magnético (línea negra). Los dos gráficos centrales muestran la conductividad del H II, mientras que los dos inferiores muestran la conductividad del He II.

óptica (en escala logarítmica) hacia el exterior de la atmósfera. Distinto es el caso de la conductividad del He II en la región interna de la atmósfera donde crece abruptamente hasta alcanzar un máximo y luego decrece suavemente hacia la superficie.

En presencia de un campo magnético se puede observar, que en las capas más internas de la atmósfera, la conductividad eléctrica calculada coincide con la conductividad en ausencia 
de campo magnético. Mientras que a partir de una dada profundidad óptica, al movernos hacia las capas exteriores de la atmósfera, la conductividad eléctrica empieza a decaer abruptamente. La profundidad óptica a la cual comienza a ocurrir este cambio varía con la intensidad del campo magnético, observándose que cuanto más intenso es el campo, más interno es el punto para el cual la conductividad eléctrica comienza a decaer.

También se observa que, para una misma intensidad de campo magnético, la disminución de la conductividad eléctrica de los electrones tiene lugar en las capas más internas que las conductividades eléctricas H II y del He II.

\subsection{Efectos de la fuerza de Lorentz sobre las distribu- ciones de presión, densidad y temperatura}

Para ejemplificar los efectos de la gravedad efectiva, y por ende de los campos magnéticos, mostraremos las distribuciones de presión, densidad y temperatura que resultan para el modelo de la estrella B2V prototipo que hemos seleccionado. Consideraremos el caso en que la latitud magnética es de $90^{\circ}$ (sobre el ecuador magnético) y los casos en que la intensidad del campo magnético es 0, 100, 1000 y 12000 Gauss. Elegimos estos valores para la intensidad del campo magnético para mostrar resultados en ausencia de campo magnético y compararlos con los casos de un campo magnético débil, uno de intensidad media, y otro intenso, éste último, más por completitud, ya que la mayoría de las estrellas Bp tiene campos de unos pocos miles de Gauss. Consideraremos, además, ambas direcciones de la fuerza de Lorentz.

En primer lugar, veremos como es afectada la presión del gas en el interior de la atmósfera en presencia de campos magnéticos. En la figura 4.10 mostramos el logaritmo de la presión (en dinas $/ \mathrm{cm}^{2}$ ) en función del logaritmo de la profundidad óptica. La línea negra representa el modelo en ausencia de campos magnéticos. Las demás, corresponden a modelos con las tres intensidades de campo magnético antes mencionadas. Para visualizar mejor en qué dirección está dirigida la fuerza de Lorentz, utilizamos líneas de trazo continuo para los casos en que la fuerza de Lorentz apunte hacia el interior de la estrella, y líneas entrecortadas para los casos en que la fuerza de Lorentz apunte hacia afuera de la estrella. Esta convención la utilizaremos en el resto de la presente sección.

Como es sabido, la presión aumenta hacia el interior de la estrella. A simple vista vemos que para una misma profundidad óptica, la presión es mayor a la del modelo sin campo magnético cuando la fuerza de Lorentz se dirige hacia adentro y es siempre menor cuando está orientada en la dirección opuesta. En cuanto a la comparación entre modelos con distintas intensidades de campo magnético, vemos que para una dada profundidad óptica, la presión del gas es mayor a mayor intensidad del campo magnético mientras que a otras 


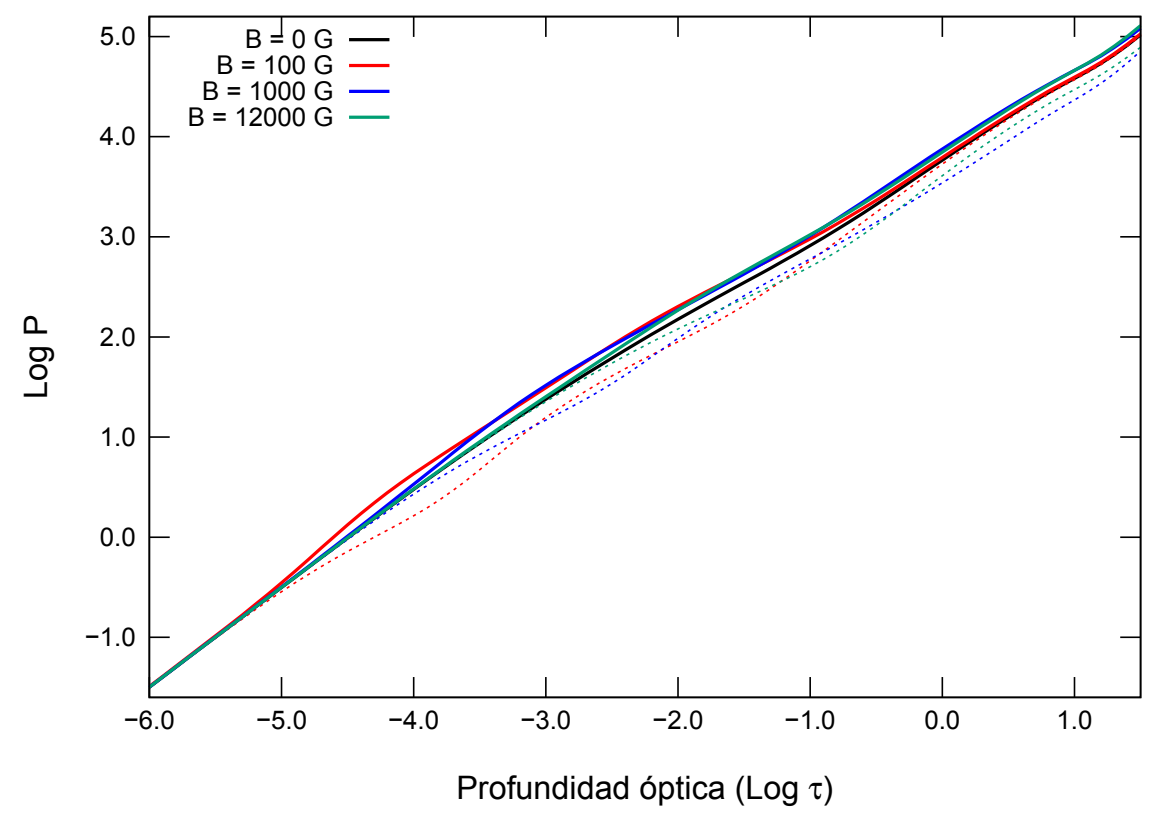

Figura 4.10: Logaritmo de la presión del gas en función del logaritmo de la profundidad óptica de Rosseland. En las líneas de trazo continuo la fuerza de Lorentz está dirigida hacia el interior de la estrella, mientras que en las líneas entrecortadas está hacia el exterior.

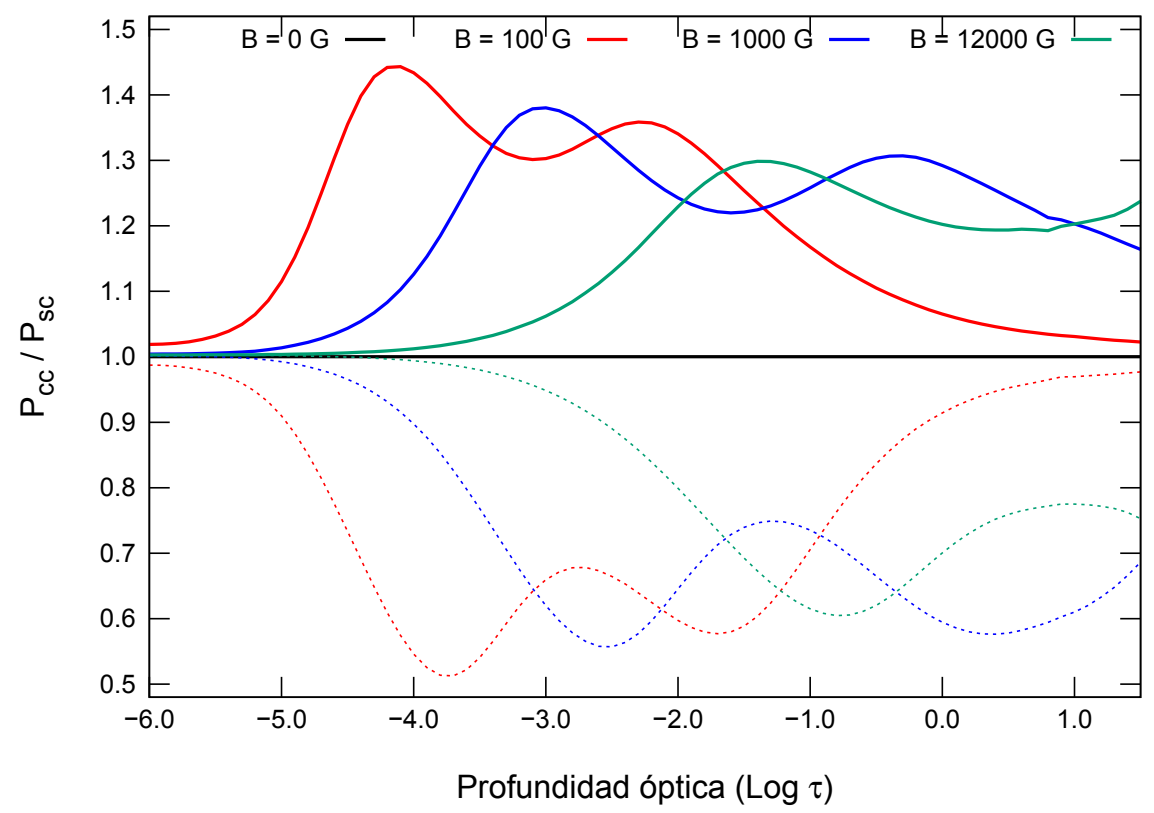

Figura 4.11: Cociente entre la presión del gas respecto a un modelos sin campo, en función del logaritmo de la profundidad óptica.

profundidades la presión de gas es mayor a menor intensidad del campo magnético.

Más información podemos obtener del cociente entre la presión de un modelo con campo magnético y otro sin campo. En la figura 4.11 se muestra este cociente para distintas intensidades del campo magnético donde claramente se observa que la presión no sigue un comportamiento monótono con la profundidad. Aquí vemos que la variación de la presión es 
mayor a casi las mismas profundidades ópticas en donde la variación de la gravedad efectiva es mayor.

A diferencia del comportamiento de la gravedad efectiva, donde la intensidad de los máximos (o mínimos según la dirección de la fuerza de Lorentz) son casi iguales, la variación relativa de la presión es menor en las capas más internas. Otra diferencia respecto al cociente $g_{e f f} / g$, es que el comportamiento no es exactamente especular, dado que los máximos relativos no ocurren a la misma profundidad óptica de los mínimos relativos si la fuerza de Lorentz está dirigida en sentido opuesto.

El mismo comportamiento se ve en el caso de la densidad, como puede apreciarse en los gráficos de la figura 4.12 dado que no hay variaciones significativas en la distribución de temperatura. Al comparar modelos con y sin campo magnético, detectamos que las variaciones de la temperatura no llegan a ser mayores que el $0.5 \%$.
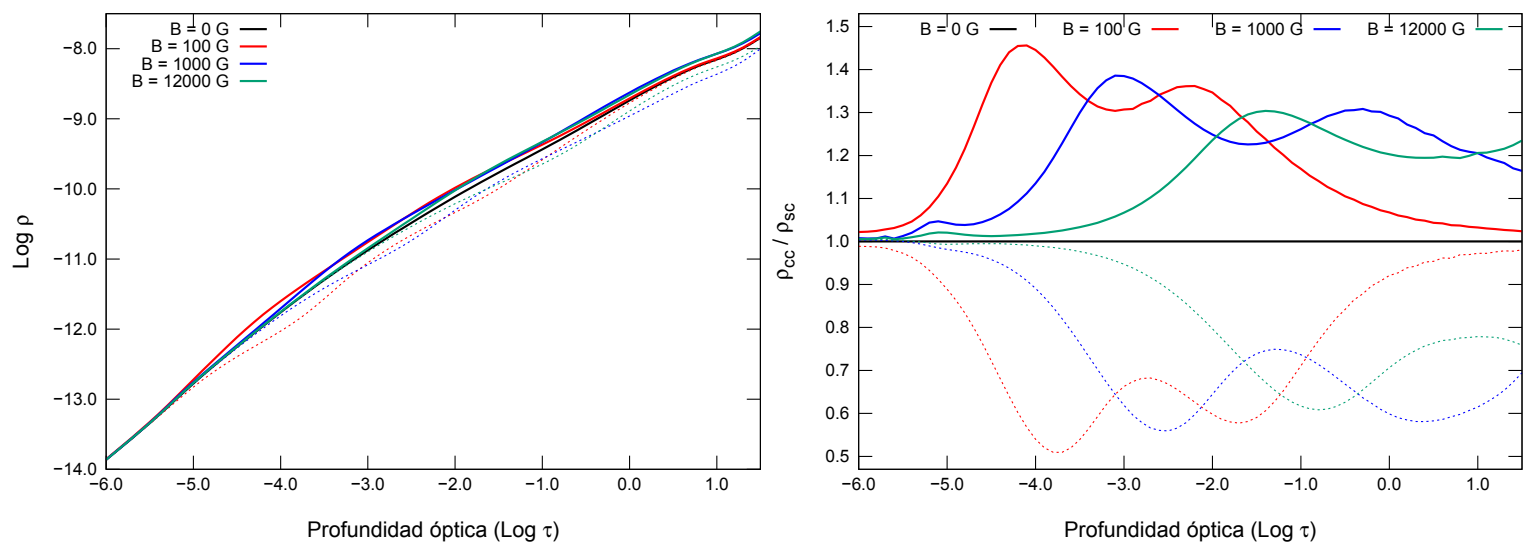

Figura 4.12: Distribución de la densidad del gas (izquierda) y cociente de la densidad del gas respecto de un modelo sin campo magnético (derecha), en función del logaritmo de la profundidad óptica.

Cabe destacar que este comportamiento es local, dado que la intensidad del campo magnético y la gravedad efectiva varían con la latitud magnética, como veremos en la siguiente sección.

\subsection{Comportamiento de la gravedad efectiva con la la- titud magnética}

En todos los casos anteriores, hemos calculado nuestros modelos considerando un ángulo latitudinal magnético de $\pi / 2$ radianes $\left(90.0^{\circ}\right)$, que corresponde al ecuador magnético, tomando como origen de este ángulo el polo norte de un dipolo magnético. El motivo de esta elección se debe a que en el ecuador magnético, los efectos de la fuerza de Lorentz sobre la gravedad efectiva son máximos, como veremos a continuación. 


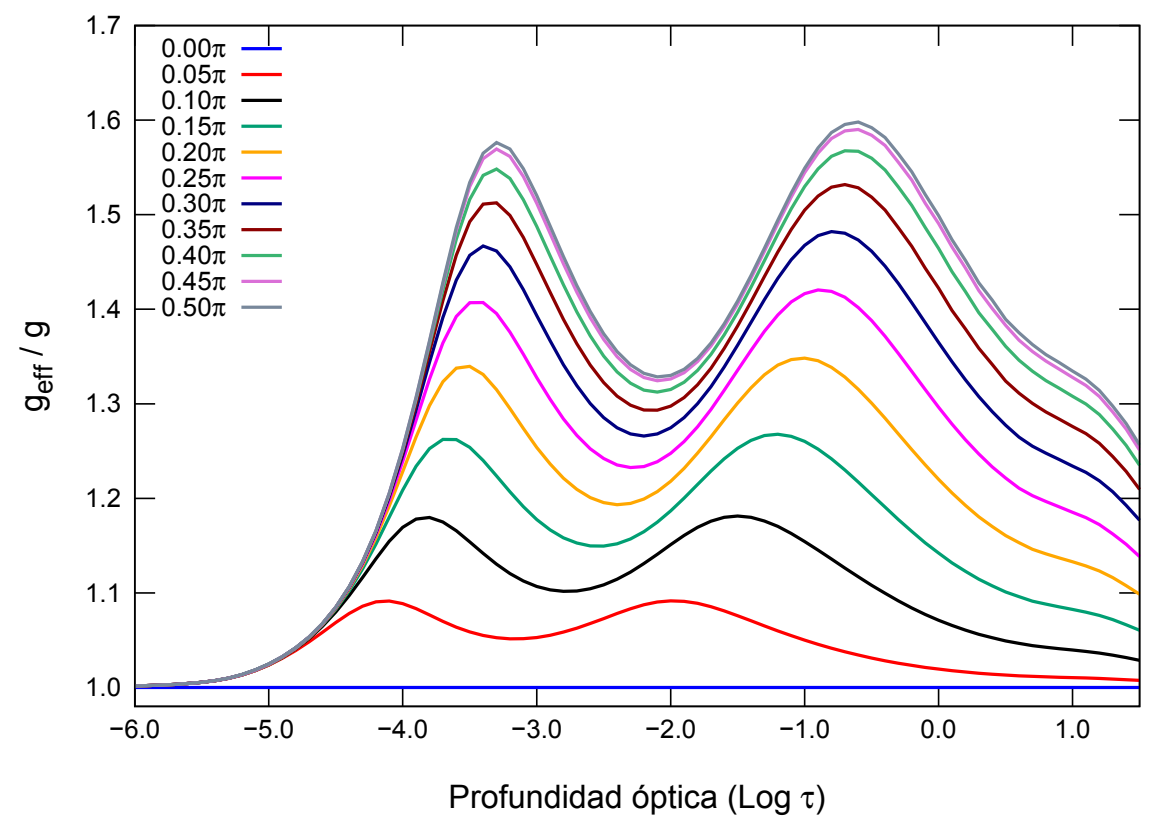

Figura 4.13: Comportamiento de la gravedad efectiva con el ángulo latitudinal magnético, desde el polo $\left(0^{\circ}\right)$ al ecuador magnético $\left(90^{\circ}\right)$.

En la figura 4.13 hemos graficado la gravedad efectiva en función del logaritmo de la profundidad óptica para un campo magnético cuya intensidad es de 1000 Gauss. Los modelos aquí mostrados se corresponden con distintos ángulos de latitud magnética entre 0 y $\pi / 2$ radianes con un paso de $\pi / 20$ radianes, esto es entre $0.0^{\circ}$ y $90.0^{\circ}$ con un paso de $9.0^{\circ}$.

Claramente vemos que en el caso de un ángulo de $90^{\circ}$ (ecuador magnético), la variación de la gravedad efectiva respecto de la gravedad superficial es máxima, mientras que en el caso de un ángulo latitudinal de $0^{\circ}$ (polo magnético), no hay variación para ninguna profundidad óptica, con lo cual la gravedad efectiva obtenida es la que tendríamos en el caso de que no haya un campo magnético.

Dada la simetría del problema, el mismo comportamiento se observará en el hemisferio comprendido entre $\operatorname{los} 90^{\circ}$ y $180^{\circ}$.

Otro efecto que vemos es que la posición de los máximos relativos varía al cambiar la latitud magnética. Para ambos máximos, estos se desplazan hacia el exterior de la atmósfera al acercarnos al polo magnético y los desplazamientos de los máximos no son iguales para cada latitud. El desplazamiento es mayor para el máximo ubicado en la región más interior, con lo cual no sólo se mueven hacia el exterior de la atmósfera, sino que también se van acercando entre sí.

Como consecuencia, este comportamiento afectará las distribuciones locales de presión y densidad, y por ende, modificará las regiones de formación de las líneas espectrales.

En los próximos capítulos (\$5 y $\$ 6$ se estudiarán qué efectos tienen estas distribuciones de 
presión y densidad sobre las líneas del átomo de He. No sólo esperaremos cambios a nivel local, sino que también a nivel global, ya que debemos tener en cuenta la orientación del eje del polo magnético respecto del eje de rotación de la estrella y la dirección de la visual de un observador externo. Los cambios globales se deberán principalmente a que un observador externo apreciará diferentes aspectos de la configuración magnética al rotar la estrella. 


\section{Capítulo 5}

\section{Efectos magnéticos locales sobre las líneas de helio}

En el capítulo anterior hemos analizado cómo la presencia de los campos magnéticos afecta la estructura de la fotosfera. Así, hemos encontrado cómo varían la gravedad efectiva, la densidad, la presión y la temperatura al cambiar la dirección de la fuerza de Lorentz y la intensidad del campo magnético. También, hemos analizado cómo es el comportamiento de estos parámetros y qué ocurre al considerar distintos ángulos de latitud magnética. Sin embargo, todas estas cantidades nos son observables directamente. En este capítulo, mostraremos cómo la fuerza de Lorentz, afecta a los perfiles de línea, en particular a las de He, que son magnitudes observables y nos permiten verificar nuestro modelo.

\subsection{El átomo de helio}

Las líneas espectrales de los distintos elementos químicos que observamos se deben a transiciones que ocurren entre estados ligados de los átomos. A grandes rasgos, la estructura interna de un átomo consiste en un núcleo central conformado por protones y neutrones, y una nube de electrones a su alrededor. La energía que puede tener un electrón está cuantificada. De esta forma, los estados de energía que puede ocupar un electrón también están cuantificados. La energía de cada estado o nivel está caracterizada por los siguientes números cuánticos: $n$ es el número cuántico principal, siendo $n=1$ el nivel fundamental; $l$ es el número cuántico orbital; $l=0,1, \ldots, n-1, m_{l}$ es la proyección del momento angular orbital, $m_{l}=-l, \ldots, 0, \ldots, l ; s$ es el momento angular de spin, $s=-1 / 2,1 / 2$. Además, por el principio de exclusión de Pauli, dos electrones no pueden tener todos los números cuánticos iguales. Puede ocurrir que para una misma energía correspondan distintas configuraciones de números cuánticos, y en ese caso se dice que está degenerada. Esta degeneración se rompe 
con el desdoblamiento de los niveles producto del acoplamiento de los momentos angulares, o la presencia de campos eléctricos o magnéticos. Los electrones en cada nivel, pueden ser descriptos mediante funciones de onda propias, que dependen de los números cuánticos, que son soluciones de la ecuación de Schrödinger del sistema.

Una transición radiativa ocurre cuando un electrón pasa de un nivel a otro absorbiendo o emitiendo fotones. Cuando el electrón pasa a un nivel inferior pierde energía liberándola en forma de un fotón, entonces observamos una línea en emisión. Cuando sube de nivel, gana energía, tomándola del campo de radiación, produciendo una línea en absorción. La longitud de onda del fotón emitido o absorbido depende de la variación de energía y está dada por,

$$
\lambda=\frac{\hbar c}{\Delta E}
$$

donde $\hbar=h / 2 \pi$, siendo $h$ la constante de Planck, $c$ es la velocidad de la luz, y $\Delta E$ es la variación de energía de la transición. El valor de la variación de energía es discreto y no puede ser cualquiera, aún estando cuantificado. Existen las llamadas reglas de selección que deben cumplirse y que indican qué variaciones de números cuánticos están permitidas. Las transiciones que cumplen estas reglas se llaman permitidas, mientras las que no, se denominan prohibidas. Las líneas prohibidas pueden aparecer, ya que las reglas de selección, establecen que tienen una baja probabilidad de ocurrir, pero ésta no es nula. También, en ciertas condiciones, como las de baja densidad de las nebulosas planetaria, esta probabilidad aumenta, permitiendo la formación de líneas prohibidas.

El átomo más simple es el del hidrógeno que posee un sólo electrón. Los demás átomos tienen una estructura más compleja, aunque los átomos que poseen $Z$ electrones y están ionizados $Z-1$ veces pueden tratarse en forma similar al átomo de $\mathrm{H}$.

El siguiente elemento en complejidad es el He. El átomo de He posee en su estado neutro dos electrones. Esto hace que a diferencia del átomo de hidrógeno, presente niveles singuletes y tripletes. Los dos electrones se consideran como idénticos, entonces podemos construir una función que satisfaga la ecuación de Schrödinger independiente del tiempo, de manera que la densidad de probabilidad permanezca invariante ante el cambio de la designación de los electrones.

Definamos primero dos funciones propias $\psi_{\alpha}(1)$ donde la partícula 1 se encuentra en el estado de los números cuánticos $\alpha$ y $\psi_{\beta}(2)$ donde la partícula 2 está en el estado $\beta$. Definimos las funciones propias $\psi_{\alpha \beta}$ con la partícula 1 en el estado $\alpha$ y la partícula 2 en el estado $\beta$, y $\psi_{\beta \alpha}$ con la partícula 1 en el estado $\beta$ y la partícula 2 en el estado $\alpha$ como, 


$$
\begin{aligned}
& \psi_{\alpha \beta}=\psi_{\alpha}(1) \psi_{\beta}(2) \\
& \psi_{\beta \alpha}=\psi_{\beta}(1) \psi_{\alpha}(2)
\end{aligned}
$$

Consideremos dos combinaciones lineales de $\psi_{\alpha \beta}$ y $\psi_{\beta \alpha}$,

$$
\begin{aligned}
\psi_{S} & =\frac{1}{\sqrt{2}}\left[\psi_{\alpha \beta}+\psi_{\beta \alpha}\right] \\
\psi_{A} & =\frac{1}{\sqrt{2}}\left[\psi_{\alpha \beta}-\psi_{\beta \alpha}\right]
\end{aligned}
$$

donde $\psi_{S}$ es una función propia simétrica y $\psi_{A}$ es una función propia antisimétrica. Así definidas, $\psi_{S}$ y $\psi_{A}$ están normalizadas. Las funciones propias $\psi_{\alpha \beta}$ y $\psi_{\beta \alpha}$ están degeneradas. La energía total no depende de que la partícula esté en un estado u otro, si las partículas son idénticas. A esta situación se denomina degeneración de intercambio. Las densidades de probabilidad de estas funciones quedan definidas como,

$$
\begin{aligned}
\psi_{S}^{*} \psi_{S} & =\frac{1}{2}\left(\psi_{\alpha \beta}^{+} \psi_{\alpha \beta}+\psi_{\beta \alpha}^{*} \psi_{\beta \alpha}\right)+\frac{1}{2}\left(\psi_{\beta \alpha}^{+} \psi_{\alpha \beta}+\psi_{\alpha \beta}^{*} \psi_{\beta \alpha}\right) \\
\psi_{A}^{*} \psi_{A} & =\frac{1}{2}\left(\psi_{\alpha \beta}^{+} \psi_{\alpha \beta}+\psi_{\beta \alpha}^{*} \psi_{\beta \alpha}\right)-\frac{1}{2}\left(\psi_{\beta \alpha}^{+} \psi_{\alpha \beta}+\psi_{\alpha \beta}^{*} \psi_{\beta \alpha}\right)
\end{aligned}
$$

Se puede ver que si realizamos los intercambios $\psi_{\alpha \beta} \rightarrow \psi_{\beta \alpha}$ y $\psi_{\beta \alpha} \rightarrow \psi_{\alpha \beta}$ las probabilidades $\psi_{S}^{*} \psi_{S} \mathrm{y} \psi_{A}^{*} \psi_{A}$ permanecen invariantes.

Consideremos ahora el caso del átomo de He, en donde las dos partículas son electrones. A las funciones propias las podemos escribir como el producto de una función de la parte espacial por una función de la parte del spin.

$$
\psi_{n l m_{l} m_{s}}=\psi_{n l m_{l}} \sigma_{m_{s}}
$$

Entonces para el electrón 1 definimos, si agrupamos a los números cuánticos espaciales $n, l$ y $m_{l}$ en el número cuántico $a$,

$$
\psi_{\alpha}(1)=\psi_{a}(1) \sigma_{m_{s}}(1)
$$

y en forma análoga para el electrón 2. Entonces para dos electrones idénticos sin interacción, como tenemos en el átomo de He, podemos escribir, 


$$
\psi_{\alpha \beta}=\psi_{\alpha}(1) \psi_{\beta}(2)=\psi_{a}(1) \psi_{b}(2) \sigma_{m_{s_{a}}} \sigma_{m_{s_{b}}}
$$

o en forma más compacta, como hicimos anteriormente,

$$
\psi_{\alpha \beta}=\psi_{a b} \sigma_{m_{s_{a}} m_{s_{b}}}
$$

Esta no es una función propia aceptable para sistemas de dos electrones ya que no es antisimétrica ante la permutación de los números 1 y 2 que designan a los electrones. Sin embargo, es posible construir una función propia antisimétrica combinando el producto de una función propia espacial simétrica o antisimétrica y una función de spin de simetría opuesta. Este producto será siempre antisimétrico. Consideremos entonces, las funciones propias de spin, y ya que para cada electrón $m_{s}$ puede tomar dos valores, $-1 / 2$ y $+1 / 2$, se tienen de esta forma cuatro posibilidades:

$$
\sigma_{+1 / 2+1 / 2}, \quad \sigma_{+1 / 2-1 / 2}, \quad \sigma_{-1 / 2+1 / 2}, \quad \sigma_{-1 / 2-1 / 2}
$$

Así podemos construir cuatro funciones propias de spin distintas que describen al sistema,

$$
\begin{aligned}
& \left.\frac{1}{\sqrt{2}}\left(\sigma_{+1 / 2-1 / 2}-\sigma_{-1 / 2+1 / 2}\right)\right\} \quad \text { Singulete } \\
& \left.\begin{array}{l}
\sigma_{+1 / 2+1 / 2} \\
\frac{1}{\sqrt{2}}\left(\sigma_{+1 / 2-1 / 2}+\sigma_{-1 / 2+1 / 2}\right) \\
\sigma_{-1 / 2-1 / 2}
\end{array}\right\} \quad \text { Triplete }
\end{aligned}
$$

Las cuatro funciones están normalizadas. A la primera se la llama función propia de spin de singulete y es antisimétrica respecto del intercambio de la designación de los electrones. Las otras tres corresponden a las funciones propias de spin de triplete y son simétricas frente al intercambio. Para que la función propia completa sea antisimétrica, a la función de spin antisimétrica debemos multiplicarla por una función espacial simétrica,

$$
\frac{1}{\sqrt{2}}\left(\psi_{a b}+\psi_{b a}\right)
$$

y a cada una de las funciones de spin simétricas debemos multiplicarla por una función espacial antisimétrica,

$$
\frac{1}{\sqrt{2}}\left(\psi_{a b}-\psi_{b a}\right)
$$

De tal manera, tenemos cuatro funciones propias completas y antisimétricas para un sistema 
formado por dos electrones sin interacción:

$$
\psi_{A}=\left\{\begin{array}{l}
\frac{1}{\sqrt{2}}\left(\psi_{a b}+\psi_{b a}\right) \frac{1}{\sqrt{2}}\left(\sigma_{+1 / 2-1 / 2}-\sigma_{-1 / 2+1 / 2}\right) \\
\frac{1}{\sqrt{2}}\left(\psi_{a b}-\psi_{b a}\right) \sigma_{+1 / 2+1 / 2} \\
\frac{1}{\sqrt{2}}\left(\psi_{a b}-\psi_{b a}\right) \frac{1}{\sqrt{2}}\left(\sigma_{+1 / 2-1 / 2}+\sigma_{-1 / 2+1 / 2}\right) \\
\frac{1}{\sqrt{2}}\left(\psi_{a b}-\psi_{b a}\right) \sigma_{-1 / 2-1 / 2}
\end{array}\right.
$$

Las 4 funciones están normalizas, son ortogonales y se encuentran degeneradas. Se puede dar una interpretación física para los estados singulete y tripletes al calcular para cada estado la magnitud y la componente $z$ del vector impulso de spin total $S^{\prime}$, que es la suma de los impulsos angulares de spin de los dos electrones,

$$
S^{\prime}=S_{1}+S_{2}
$$

Se encuentra que la magnitud y la componente $z$ del vector impulso angular total de spin son,

$$
S^{\prime}=\sqrt{s^{\prime}\left(s^{\prime}+1\right)} \hbar S_{z}^{\prime}=m_{s}^{\prime} \hbar
$$

donde $m_{s}^{\prime}=-s^{\prime}, \ldots .,+s^{\prime}$ y $s^{\prime}=0,1$ para los estados de singulete y tripletes, respectivamente. Esto se puede interpretar como que restamos o sumamos dos vectores de impulso de spin de modulo $1 / 2$, resultando 0 ó 1 , respectivamente. Tenemos que para el estado de singulete, $m_{s}^{\prime}=0$. Para las funciones propias de spin $\sigma_{+1 / 2+1 / 2}$ y $\sigma_{-1 / 2-1 / 2}$, se ve que el módulo del vector total es 1 y que las componentes $z$ de dichos vectores son $m_{s}^{\prime}= \pm 1$. En cambio, para las funciones $\frac{1}{\sqrt{2}}\left(\sigma_{+1 / 2-1 / 2}-\sigma_{-1 / 2+1 / 2}\right)$ y $\frac{1}{\sqrt{2}}\left(\sigma_{+1 / 2-1 / 2}-\sigma_{-1 / 2+1 / 2}\right)$ no es obvio que $S^{\prime}=0$ para el estado antisimétrico y $S^{\prime}=1$ para el estado simétrico.

La existencia de los estados tripletes hace que exista una fuerza de intercambio que es netamente cuántica. Esto hace que al momento de formarse las líneas, en las transiciones que involucran estados de tripletes, no aparezca sólo una componente de la línea, sino que además, aparecen componentes de menor intensidad con longitudes de onda levemente desplazadas. Dependiendo de la resolución del detector, estas componentes se ven claramente o se solapan viéndose como un ensanchamiento de la transición de la línea principal.

En la figura 5.1 mostramos el esquema de niveles y las transiciones mas probables del átomo de He. 


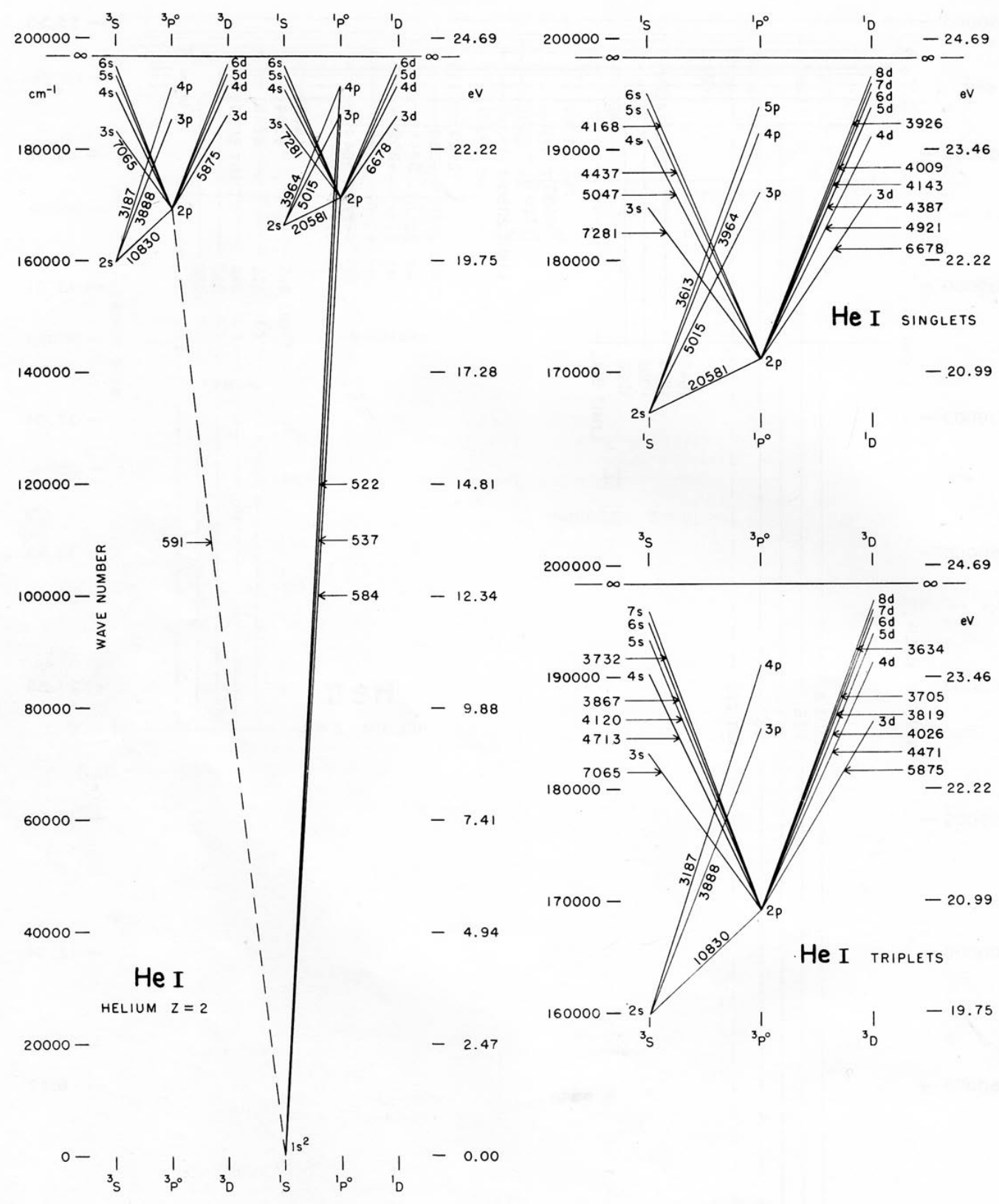

Figura 5.1: Grotriano del átomo de He con sus niveles y sus respectivas transiciones.

\subsection{Modelado de perfiles de líneas}

\subsubsection{Modelado de líneas en general}

Modelaremos la opacidad en las líneas considerando una atmósfera en equilibrio termodinámico local (ETL). Para poder calcular las poblaciones de los distintos estados excitados de cada especie química, se utiliza la ley de Saha-Boltzmann, y a partir de esos valores calcularemos los perfiles de línea correspondientes a cada transición.

Para modelar las líneas hemos utilizados perfiles Lorentzianos siguiendo los lineamientos del trabajo de Anderson 1989. Siendo $\sigma_{\nu}$ el perfil de la línea, el mismo queda definido por, 


$$
\sigma_{\nu}=\frac{\gamma}{\pi\left[\left(\nu-\nu_{0}\right)^{2}+\gamma^{2}\right]}
$$

donde $\nu_{0}$ es la frecuencia central de la línea. El ensanchamiento total de la línea es la suma del ensanchamiento natural, del ensanchamiento debido al efecto Stark lineal y del

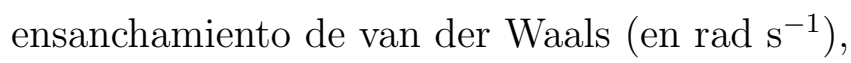

$$
\gamma=\frac{\gamma_{\text {nat }}+\gamma_{\text {Stark }} / f+\gamma_{v d W}}{4 \pi}
$$

El ensanchamiento por efecto Stark lineal es ajustado según los resultados de Griem 1960, mientras que el ensanchamiento de van der Waals es ajustado a las transiciones $d-f$ del trabajo de Deridder y van Renspergen 1976. Para temperaturas mayores a $10000 \mathrm{~K}$, debido a la ionización, el ensanchamiento Stark debe dividirse por un factor $f$ que en nuestro caso consideramos igual a 3 .

Para el caso de las líneas de He I $\lambda 5015$ y He I $\lambda 5875$, debido a las colisiones, debemos considerar el ensanchamiento Doppler, obteniendo perfiles de Voigt, adoptando la aproximación del efecto Stark según el trabajo de Dimitrijevic y Sahal-Brechot 1990.

\subsubsection{Modelado de líneas con componentes prohibidas}

Algunas estrellas peculiares en He, muestran intensas líneas prohibidas de He, superpuestas a las líneas de He permitidas. Algunos ejemplos de líneas que presentan una componente prohibida son: He I $\lambda 4026$, He I $\lambda 4471$, He I $\lambda 4922$ y He I $\lambda 4009$. Estas transiciones en realidad, no son componentes prohibidas propiamente dichas, como las que presentan las nebulosas planetarias y que se originan en plasmas poco densos, sino líneas de intercombinación que se producen en plasma de alta densidad como lo son las fotosferas de las estrellas B peculiares (Bp), las estrellas Be y algunas supergigantes.

Desde hace bastante tiempo se sabe de la aparición de líneas prohibidas del He, tales como He I $\lambda 4470.0 \AA{ }^{\circ}\left(2^{3} \mathrm{P}-4^{3} \mathrm{~F}\right)$, ocurre en la atmósfera estelar a partir de la ruptura de las reglas de selección del dipolo en presencia de una perturbación del campo eléctrico. En este caso, podemos utilizar procedimientos de solapamiento de líneas para determinar los perfiles completamente.

Las componentes permitidas y prohibidas de las líneas de He son modeladas mediante la expresión dada por Griem 1968); Gieske y Griem 1969); Griem 1974:

$$
I(\lambda)=\frac{P_{P}}{\pi} \frac{\omega}{\omega^{2}+\left(\lambda-\lambda_{P}\right)^{2}}+\frac{P_{R}}{\pi} \frac{\omega}{\omega^{2}+\left(\lambda-\lambda_{P}-d\right)^{2}},
$$


donde $\omega$ es el ensanchamiento de la línea por efecto Stark, $\lambda_{P}$ es la longitud de onda de la componente permitida, y $d$ es el desplazamiento de la componente prohibida respecto de la permitida. $P_{P}$ y $P_{R}$ son los pesos estadísticos de las componentes permitida y prohibida, respectivamente. Para realizar los cálculos, los $\omega$ son obtenidos por interpolación lineal, mientras que los pesos $P_{P}$ y $P_{R}$ son estimados a partir de los espectros observados

\subsection{Efectos de la fuerza de Lorentz sobre las líneas de helio y sus anchos equivalentes}

El código que utilizamos para resolver la estructura de la atmósfera Rohrmann 2001 también nos permite determinar los perfiles de línea de $\mathrm{H}$ y $\mathrm{He}$, tal como se describe en el anexo A.1. Para poder comparar las variaciones espectrales en líneas que resultan de otros modelos y, eventualmente, con las observaciones, debemos normalizar estos perfiles mediante el programa descripto en el anexo A.4 Además, junto con el perfil normalizado, obtenemos el ancho equivalente del perfil, así como la intensidad en el centro de la línea.

En esta sección mostraremos los perfiles de líneas locales obtenidos para modelos de estrellas de tipo espectral B2 en presencia de una fuerza de Lorentz que varía con la latitud magnética. En todos los casos, consideraremos al campo eléctrico constante, $E_{e q}=5 \times 10^{-11} \mathrm{~cm}^{-1 / 2} \mathrm{~g}^{1 / 2}$ $\mathrm{s}^{-1}$. Valores más pequeños de $E_{e q}$ no tienen influencia sobre la estructura de la atmósfera, mientras que valores mayores de $E_{e q}$ conducen a soluciones inestables (por ejemplo, con $E_{e q} \sim 10^{-10} \mathrm{~cm}^{-1 / 2} \mathrm{~g}^{1 / 2} \mathrm{~s}^{-1}$, se incrementa la gravedad efectiva en la superficie en un factor 5). Sin embargo, el valor adoptado puede ser comparado con el campo eléctrico inducido producto del decaimiento del campo magnético dipolar fósil, y una estimación aproximada de este valor podría dar indicios sobre su origen. Entonces, teniendo en cuenta que las observaciones indican campos magnéticos de unos pocos kiloGauss (Petit et al. 2013, y suponiendo que existen configuraciones del campo magnético global estelar estables para intensidades de más de 300 Gauss Aurière et al. 2007a, calculamos $E_{\text {eq }} \sim R_{*} B_{0} /$ ct para $B_{0} \sim 1 k G, R_{*}=5.2 R_{\odot}$, y un tiempo característico para el decaimiento del campo magnético de $t \sim 3.3 \times 10^{9}$ años $\left(t \sim 4 \pi \sigma_{i} R^{2} / c^{2}\right.$ con $\sigma_{i}=5.6 \times 10^{13}$ en unidades CGS, Landstreet, 1987, obtenemos $E_{e q} \sim 1.2 \times 10^{-13} \mathrm{~cm}^{-1 / 2} \mathrm{~g}^{1 / 2} \mathrm{~s}^{-1}$. Este valor es dos órdenes de magnitud inferior al $E_{e q}$ necesario para producir efectos de la presión magnética en la estructura atmosférica. Por lo tanto, diferentes mecanismos deben ser tenidos en cuenta para producir corrientes atmosféricas significativas en las estrellas Ap / Bp, como discute Shulyak et al. 2010.

En las figuras 5.2, 5.3, 5.4, 5.5 y 5.6, graficamos los perfiles correspondientes a las líneas He I $\lambda 4026, \lambda 4471, \lambda 4921, \lambda 5015$ y $\lambda 5875$, respectivamente. En estas cinco figuras, mostramos el perfil de la línea para un campo magnético con una intensidad de 1000 Gauss con la fuerza 

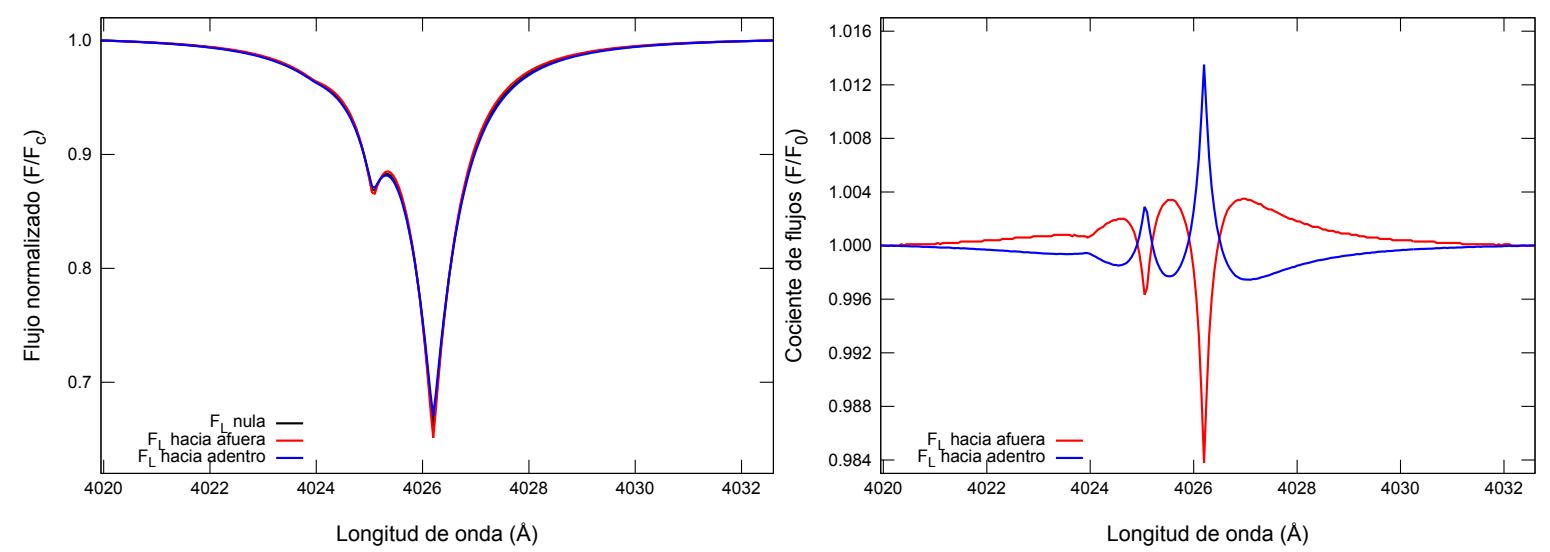

Figura 5.2: Perfil normalizado de la línea He I $\lambda$ 4026. Graficamos el perfil correspondiente a un modelo sin campo magnético (negro), y con un campo de $1000 \mathrm{G}$ con la $F_{L}$ dirigida hacia afuera (rojo), y con $F_{L}$ dirigida hacia adentro (azul). A la derecha se muestra el cociente entre los flujos con y sin campo magnético.
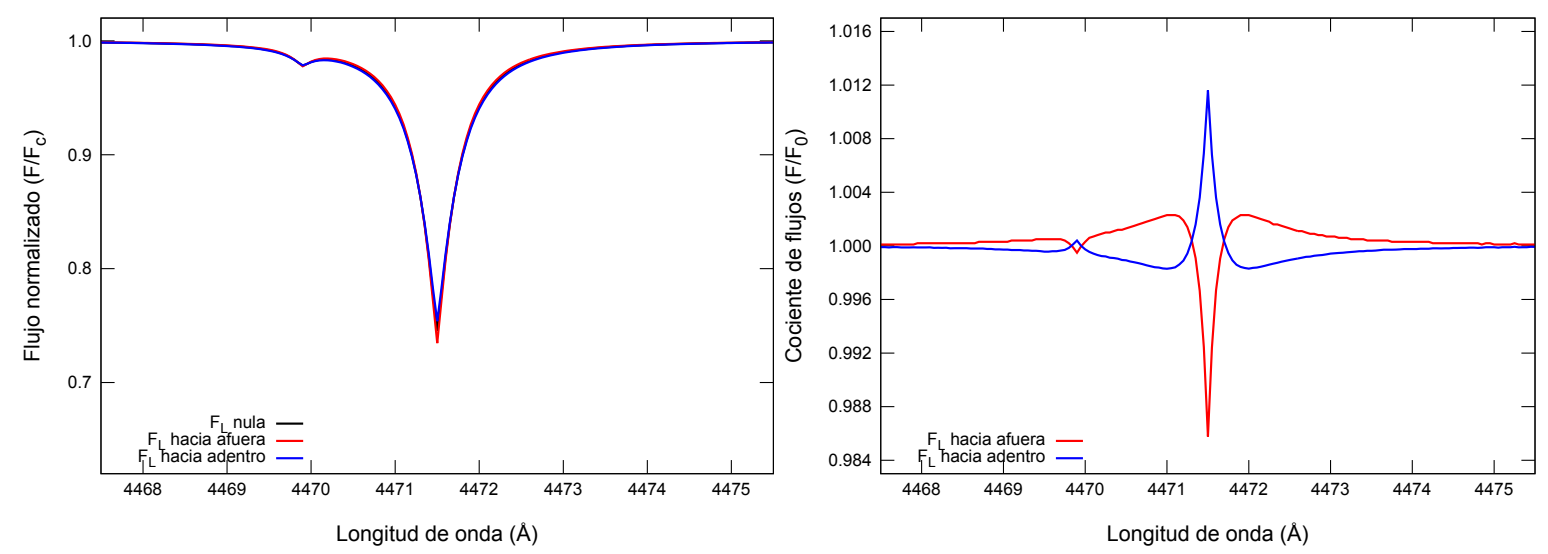

Figura 5.3: Perfil normalizado de la línea He I $\lambda$ 4471. Graficamos el perfil correspondiente a un modelo sin campo magnético (negro), y con un campo de $1000 \mathrm{G}$ con la $F_{L}$ dirigida hacia afuera (rojo), y con $F_{L}$ dirigida hacia adentro (azul). A la derecha se muestra el cociente entre los flujos con y sin campo magnético.

de Lorentz dirigida hacia el interior (líneas azules) y hacia el exterior (líneas rojas). A modo de referencia graficamos también el perfil correspondiente a un modelo sin campo magnético (líneas negras).

Si bien las variaciones relativas son pequeñas, se puede observar que en el caso en que la fuerza de Lorentz está dirigida hacia el exterior, la línea aparece más intensa que su contraparte sin campo magnético, y en la región de las alas aparece más delgada. Todo lo contrario ocurre cuando la fuerza de Lorentz apunta hacia el interior de la estrella. Aquí, la línea aparece menos intensa y las alas son más extendidas.

Dado que las variaciones son pequeñas, en los gráficos es difícil de observar este efecto, por ello en los paneles derechos de las figuras 5.2, 5.3, 5.4, 5.5, y 5.6, graficamos el cociente entre la línea del modelo con campo magnético y la línea del modelo sin campo magnético. En ellos, las variaciones antes descriptas se hacen más evidentes. 

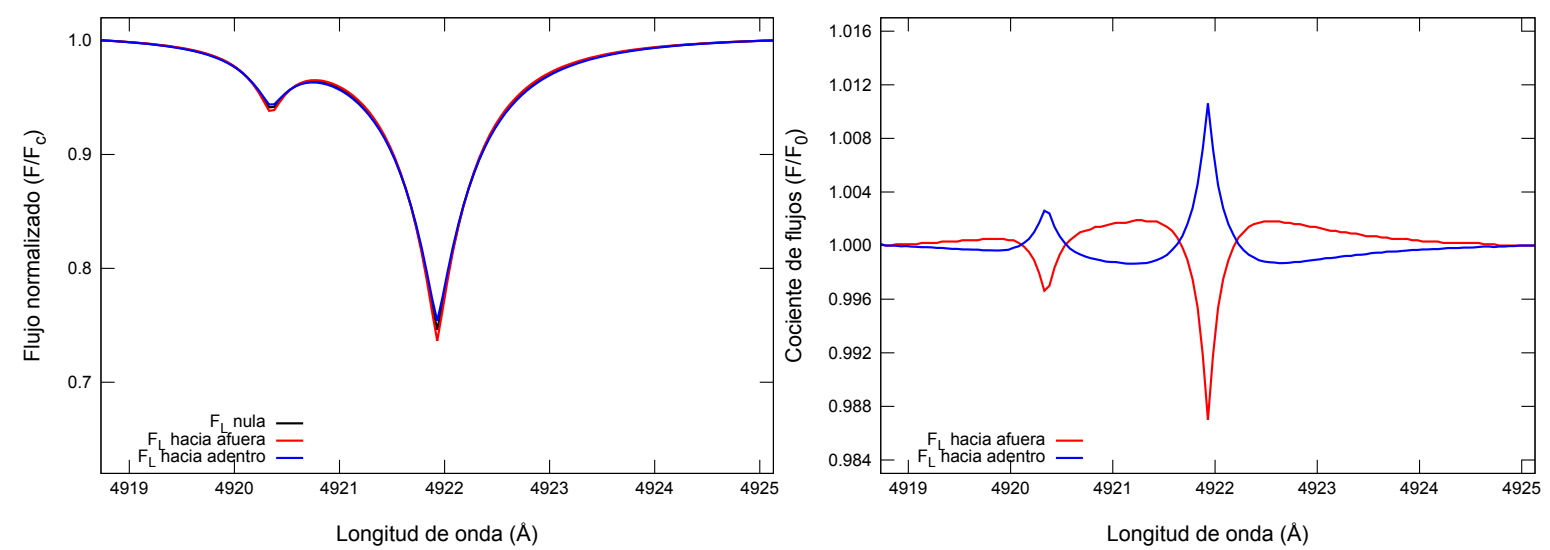

Figura 5.4: Perfil normalizado de la línea He I $\lambda$ 4921. Graficamos el perfil correspondiente a un modelo sin campo magnético (negro), y con un campo de $1000 \mathrm{G}$ con la $F_{L}$ dirigida hacia afuera (rojo), y con $F_{L}$ dirigida hacia adentro (azul). A la derecha se muestra el cociente entre los flujos con y sin campo magnético.

Observamos, además, que la variación en la intensidad de la línea no es la misma si la dirección de la fuerza de Lorentz cambia. Se observa que es mayor cuando la fuerza de Lorentz se dirige hacia el exterior de la estrella. Además, dependiendo de la línea, la variación de la intensidad en el centro de la línea es mayor que en las alas, o viceversa. Además, podemos derivar de la variación relativa de la intensidad respecto del modelo sin campo magnético la dirección de la fuerza de Lorentz.

En el caso de He I $\lambda 4026$ (figura 5.2), vemos que la variación en la intensidad respecto al modelo sin campo magnético llega al $1.6 \%$ en el caso en que la fuerza de Lorentz apunta hacia el exterior, mientras que si apunta hacia el interior, la variación es aproximadamente del $1.4 \%$. Aquí, la variación en las alas es menor, llegando a ser a lo sumo un $0.4 \%$ cuando la fuerza de Lorentz es hacia afuera. En cuanto a la componente prohibida, la variación en el centro es comparable a la variación en las alas.

Para He I $\lambda 4471$ (figura 5.3), la variación en la intensidad en el centro es menor, tomando valores entre el $1.4 \%$ y $1.2 \%$ dependiendo de la dirección de la fuerza de Lorentz. En el caso de las alas, la variación no llega al $0.2 \%$. La variación de la intensidad del centro de la componente prohibida en esta línea es pequeña, incluso bastante menor que la de las alas de la componente permitida.

El caso de He I $\lambda 4921$ (figura 5.4, es similar al de He I $\lambda 4026$, con variaciones de $1.3 \%$ y $1.1 \%$ en la intensidad del centro de la línea y de aproximadamente un $0.2 \%$ en las alas. Aquí la variación de intensidad de la componente prohibida es mayor que la de las alas, llegando a ser de casi un $0.4 \%$.

En los casos de He I $\lambda 5015$ y He I $\lambda 5875$ (figuras 5.5 y 5.6 respectivamente), el comportamiento es similar. Aquí, la variación de intensidad en el centro de la línea es menor al 

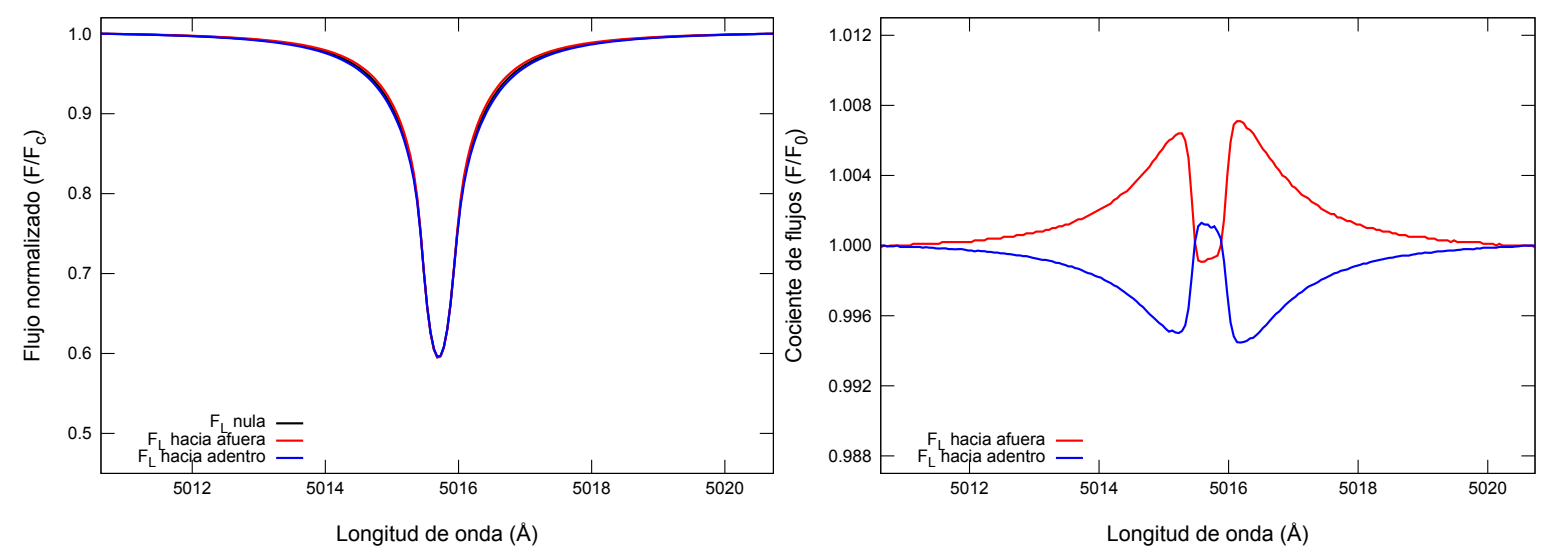

Figura 5.5: Perfil normalizado de la línea He I $\lambda 5015$ (izquierda). Graficamos el perfil correspondiente a un modelo sin campo magnético (negro), y un campo de $1000 \mathrm{G}$ con $F_{L}$ dirigida hacia afuera (rojo), y con $F_{L}$ dirigida hacia adentro (azul). A la derecha se muestra el cociente entre los flujos con y sin campo magnético.
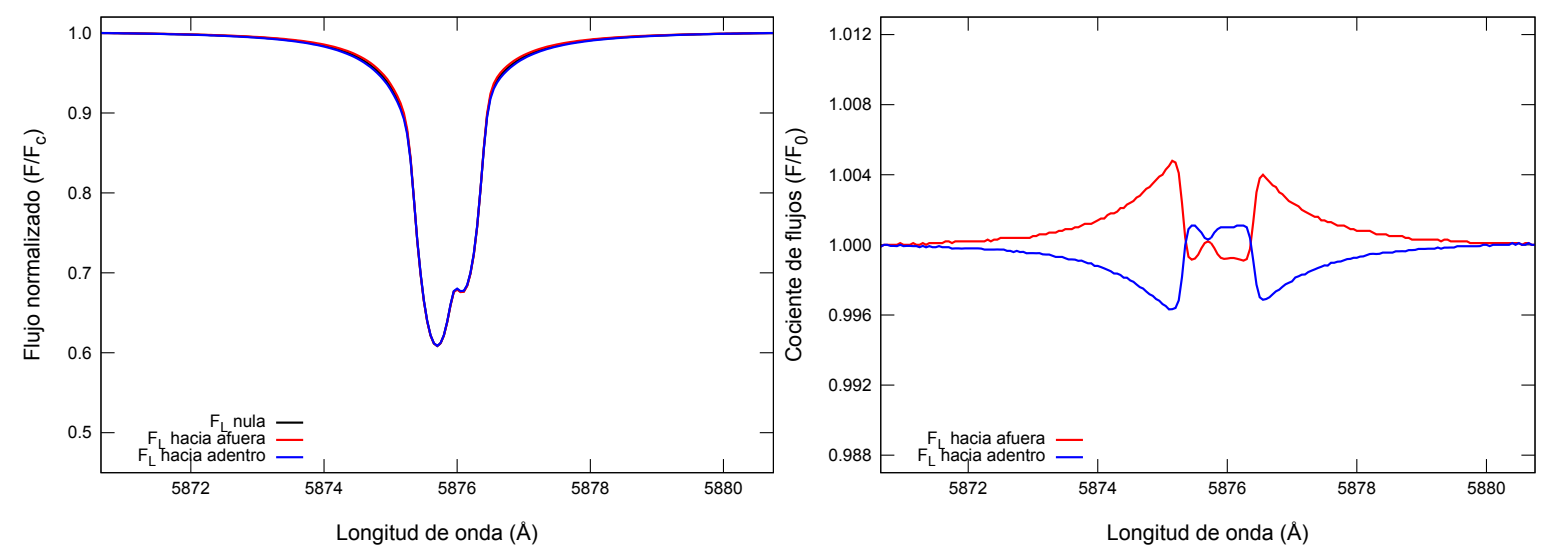

Figura 5.6: Perfil normalizado de la línea He I $\lambda 5875$ (izquierda). Graficamos el perfil correspondiente a un modelo sin campo magnético (negro), y un campo de $1000 \mathrm{G}$ con $F_{L}$ dirigida hacia afuera (rojo), y con $F_{L}$ dirigida hacia adentro (azul). A la derecha se muestra el cociente entre los flujos con y sin campo magnético.

0.1\%, mientras la variación en las alas es notablemente mayor, siendo de un $0.7 \%$ para He I $\lambda 5015$ y un poco más de $0.4 \%$ para He I $\lambda 5015$.

Aquí, se aprecia que, la variación de la intensidad de las líneas respecto del modelo sin campo magnético es en general a lo sumo del orden de un 1.6\%, en algunas líneas de He y que si comparamos modelos con la fuerza de Lorentz en direcciones opuestas, la variación no llegaría a ser mayor a un 3.0\%. Si bien en algunas estrellas CP las variaciones de sus perfiles son de ese orden, en muchas otras, esto no alcanzaría para explicar las variaciones observadas.

En todos estos gráficos hemos utilizado la misma escala en los cocientes de flujos para poder realizar una mejor comparación entre las distintas líneas.

Todos estos cálculos los hemos realizado también para otras intensidades del campo magnético, tanto menores como mayores a los 1000 Gauss. Encontramos que el comportamiento es si- 
milar y que las magnitudes de las variaciones en la intensidad son parecidas al caso de 1000 Gauss. A modo de ejemplo describiremos algunos resultados para un campo magnético de una intensidad de 200 Gauss, aunque no mostraremos aquí los correspondientes gráficos ya que visualmente son prácticamente iguales a los de 1000 Gauss.

Para campos magnéticos se 200 Gauss de intensidad, vimos que en el caso de He i $\lambda 4026$ la variación en la intensidad en el centro de la línea es similar al caso de los modelos con campos magnéticos de 1000 Gauss. Para esta intensidad del campo magnético, las variaciones son mayores, teniendo para el centro de la línea variaciones de hasta 1.8\% cuando la fuerza de Lorentz apunta hacia el exterior y 1.5\% cuando apunta hacia el interior. La variación de la intensidad en las alas de la línea también es mayor, llegando a ser de casi un $0.6 \%$ cuando la fuerza de Lorentz apunta hacia el exterior de la estrella.

En cuanto al caso de He I $\lambda 4471$, vimos que la variación de la intensidad en el centro y en las alas es también mayor que en el caso de 1000 Gauss llegando hasta casi un $1.6 \%$ con la fuerza de Lorentz apuntando hacia afuera.

Para He I $\lambda$ 4921, las variaciones en intensidad son también mayores al caso de 1000 Gauss, llegando a un 1.4\% máximo en el centro de la línea. Aquí también se observa que la variación de la componente prohibida es mayor que en las alas de la componente permitida, a diferencia de He I $\lambda 4471$, donde la variación en la intensidad en el centro de la línea prohibida es mucho menor que en las alas, o del caso de He I $\lambda 4026$ donde las variaciones de intensidad son comparables.

En lo referente a He I $\lambda 5015$ y He I $\lambda 5875$, en ambas se observa una mayor variación en la intensidad en la zona de las alas de las líneas.

Para completar el estudio de los perfiles de línea locales, analizaremos como varían los anchos equivalentes de los mismos en función de la intensidad del campo magnético y de la dirección de la fuerza de Lorentz.

En la tabla 5.1. listamos los anchos equivalentes de las cinco líneas que estamos presentando para distintas intensidades del campo magnético, y con la fuerza de Lorentz dirigida hacia el exterior de la estrella. Por otro lado, en la tabla 5.2. mostramos los anchos equivalentes, pero con la fuerza de Lorentz apuntando hacia el interior de la estrella.

En las figuras 5.7 y 5.8, graficamos los anchos equivalentes de las líneas de He en función de la intensidad del campo magnético. En los gráficos, además, se muestran los dos casos posibles de la dirección de la fuerza de Lorentz. En rojo, cuando la fuerza apunta hacia el exterior y en azul cuando apunta hacia interior de la estrella, siguiendo la misma convención de colores que veníamos usando.

En todos los casos se observa que cuando la fuerza de Lorentz es hacia el interior de la estrella, 
Tabla 5.1: Anchos equivalentes $(\AA)$ de distintas líneas de He I, para diferentes intensidades de campos magnéticos, con la fuerza de Lorentz dirigida hacia el exterior.

\begin{tabular}{||l|l|l|l|l|l||}
\hline B (Gauss) & He I $\lambda 4026.20$ & He I $\lambda 4471.50$ & He I $\lambda 4921.93$ & He I $\lambda 5015.68$ & He I $\lambda 5875.64$ \\
\hline \hline 0 & 0.5451 & 0.2111 & 0.2762 & 0.4588 & 0.4793 \\
\hline 3 & 0.5446 & 0.2108 & 0.2759 & 0.4579 & 0.4787 \\
\hline 5 & 0.5443 & 0.2107 & 0.2759 & 0.4572 & 0.4786 \\
\hline 10 & 0.5435 & 0.2103 & 0.2757 & 0.4554 & 0.4771 \\
\hline 50 & 0.5390 & 0.2081 & 0.2743 & 0.4452 & 0.4710 \\
\hline 100 & 0.5356 & 0.2063 & 0.2735 & 0.4387 & 0.4677 \\
\hline 200 & 0.5334 & 0.2053 & 0.2732 & 0.4359 & 0.4669 \\
\hline 300 & 0.5338 & 0.2052 & 0.2738 & 0.4372 & 0.4673 \\
\hline 400 & 0.5347 & 0.2058 & 0.2741 & 0.4389 & 0.4688 \\
\hline 500 & 0.5356 & 0.2062 & 0.2744 & 0.4405 & 0.4697 \\
\hline 750 & 0.5370 & 0.2068 & 0.2746 & 0.4427 & 0.4704 \\
\hline 1000 & 0.5375 & 0.2071 & 0.2747 & 0.4434 & 0.4707 \\
\hline 3000 & 0.5362 & 0.2066 & 0.2739 & 0.4400 & 0.4682 \\
\hline 6000 & 0.5344 & 0.2054 & 0.2734 & 0.4368 & 0.4668 \\
\hline 12000 & 0.5347 & 0.2055 & 0.2741 & 0.4386 & 0.4687 \\
\hline 18000 & 0.5365 & 0.2067 & 0.2748 & 0.4421 & 0.4706 \\
\hline 50000 & 0.5415 & 0.2090 & 0.2759 & 0.4512 & 0.4753 \\
\hline
\end{tabular}

Tabla 5.2: Anchos equivalentes $(\AA)$ de distintas líneas de He I, para diferentes intensidades de campos magnéticos, con la fuerza de Lorentz dirigida hacia el interior.

\begin{tabular}{||l|l|l|l|l|l||}
\hline B (Gauss) & He I $\lambda 4026.20$ & He I $\lambda 4471.50$ & He I $\lambda 4921.93$ & He I $\lambda 5015.68$ & He I $\lambda 5875.64$ \\
\hline \hline 0 & 0.5451 & 0.2111 & 0.2762 & 0.4588 & 0.4793 \\
\hline 3 & 0.5457 & 0.2111 & 0.2763 & 0.4600 & 0.4795 \\
\hline 5 & 0.5458 & 0.2113 & 0.2763 & 0.4603 & 0.4800 \\
\hline 10 & 0.5463 & 0.2116 & 0.2764 & 0.4616 & 0.4806 \\
\hline 50 & 0.5488 & 0.2129 & 0.2769 & 0.4690 & 0.4850 \\
\hline 100 & 0.5504 & 0.2136 & 0.2769 & 0.4733 & 0.4876 \\
\hline 200 & 0.5516 & 0.2143 & 0.2771 & 0.4761 & 0.4891 \\
\hline 300 & 0.5518 & 0.2147 & 0.2770 & 0.4761 & 0.4889 \\
\hline 400 & 0.5518 & 0.2145 & 0.2769 & 0.4753 & 0.4881 \\
\hline 500 & 0.5515 & 0.2144 & 0.2768 & 0.4743 & 0.4877 \\
\hline 750 & 0.5507 & 0.2140 & 0.2767 & 0.4730 & 0.4868 \\
\hline 1000 & 0.5503 & 0.2138 & 0.2767 & 0.4720 & 0.4862 \\
\hline 3000 & 0.5501 & 0.2138 & 0.2768 & 0.4732 & 0.4871 \\
\hline 6000 & 0.5511 & 0.2140 & 0.2769 & 0.4756 & 0.4887 \\
\hline 12000 & 0.5515 & 0.2145 & 0.2769 & 0.4754 & 0.4883 \\
\hline 18000 & 0.5511 & 0.2142 & 0.2768 & 0.4733 & 0.4872 \\
\hline 50000 & 0.5484 & 0.2129 & 0.2763 & 0.4663 & 0.4829 \\
\hline \hline
\end{tabular}

el ancho equivalente de la línea aumenta con la intensidad del campo, hasta alcanzar un valor máximo cuando la intensidad del campo es de 200 a 300 Gauss, y luego disminuye un poco, permaneciendo luego casi constante al ir aumentando la intensidad el campo magnético. 


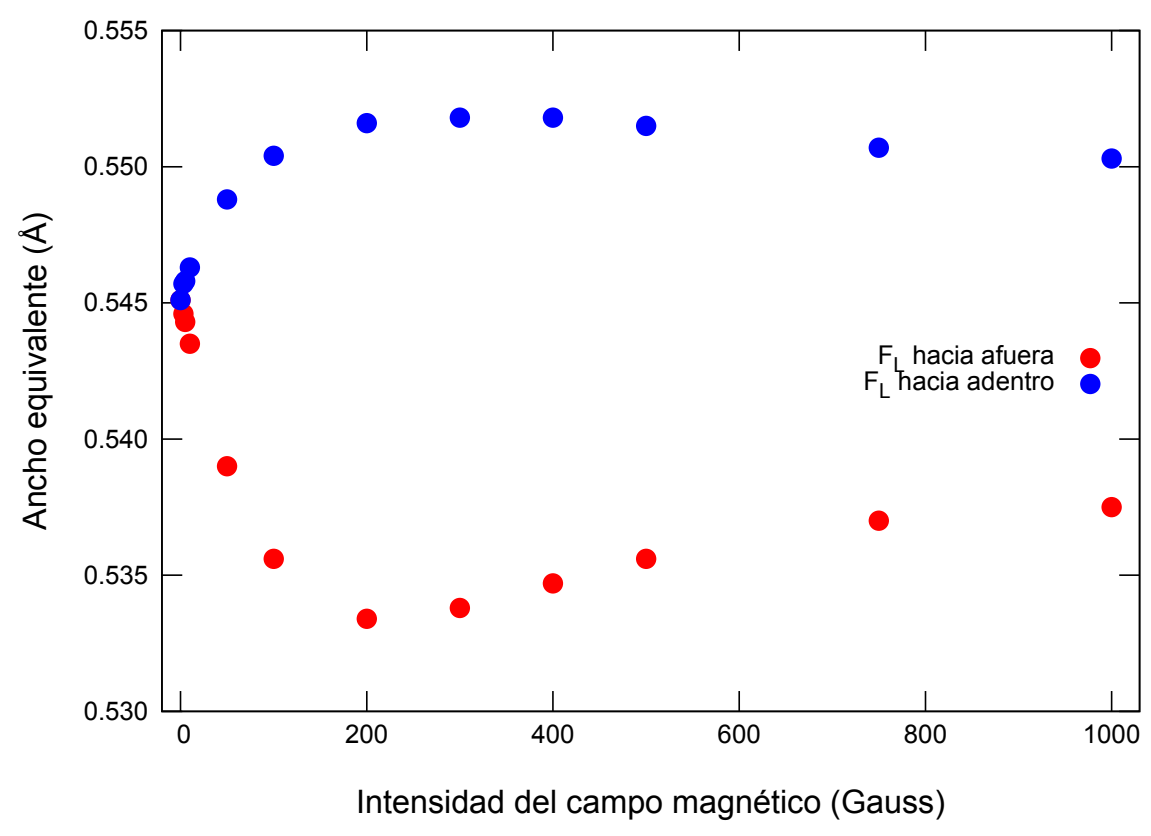

Figura 5.7: Anchos equivalentes de la línea He I $\lambda 4026$ para distintos valores de la intensidad del campo magnético. Los puntos azules corresponden a modelos con la $F_{L}$ dirigida al interior, mientras que los rojos corresponde al caso de la $F_{L}$ dirigida hacia exterior.

En el caso de que la fuerza de Lorentz apunte hacia el exterior de la estrella, el comportamiento es exactamente al revés, disminuyendo hasta alcanzar un mínimo, y luego aumentando levemente para ser casi constante al ir aumentando la intensidad del campo magnético. El valor mínimo también ocurre cuando la intensidad del campo magnético es de unos 200 300 Gauss.

De estos gráficos se desprende que el efecto de la presencia de campos magnéticos sobre los anchos equivalentes y sobre la forma e intensidad de las líneas es mayor cuando las intensidades de los mismos son de unos pocos de cientos de Gauss, y por más que tengamos campos mucho más intensos, si los hubiera, no tendríamos mayores variaciones en los perfiles de las líneas.

Otra característica que observamos, es que en todos los casos las variaciones en el ancho equivalente de las líneas es mayor cuando la fuerza de Lorentz apunta hacia el exterior de la estrella.

Cuantitativamente, encontramos que para He I $\lambda 4026$ la variación del ancho equivalente respecto a la línea sin campo magnético es de hasta un $2.2 \%$ cuando la fuerza de Lorentz apunta hacia el exterior de la estrella, y para un campo magnético de 200 Gauss, que es cuando el efecto es máximo. Cuando la fuerza de Lorentz apunta hacia el interior, la variación puede ser de hasta un 1.2\%. En el caso de He I $\lambda 4471$, las variaciones alcanzan valores de $2.7 \%$ y $1.5 \%$, respectivamente, mientras que para He I $\lambda 4921$, las variaciones son menores, $1.1 \%$ y $0.3 \%$. Estas tres líneas poseen componentes prohibidas. 

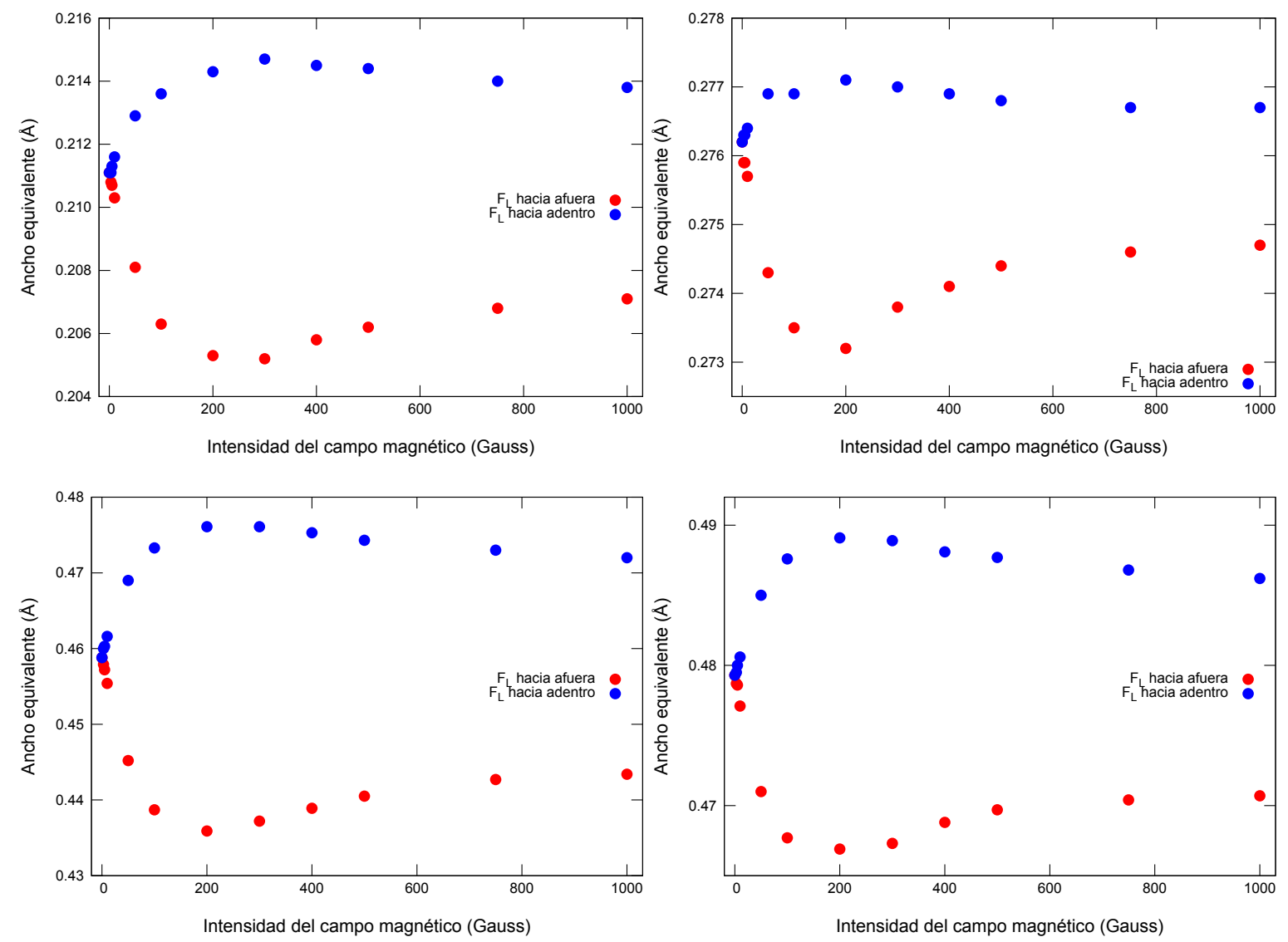

Figura 5.8: Anchos equivalentes de las líneas He I $\lambda 4471$ (arriba, izquierda), He I $\lambda 4921$ (arriba, derecha), He I $\lambda 5015$ (abajo, izquierda) y He I $\lambda 5875$ (abajo, derecha) para distintos valores de la intensidad del campo magnético. Los puntos azules corresponden a modelos con la $F_{L}$ dirigida al interior, mientras que los rojos corresponde al caso de la $F_{L}$ dirigida hacia exterior.

Distinto es el caso de He I $\lambda 5015$ y de He I $\lambda 5875$, que no poseen componentes prohibidas, y las variaciones en los anchos equivalentes son mayores. Para He I $\lambda 5015$, las variaciones llegan a ser del $5.0 \%$ cuando la fuerza de Lorentz apunta hacia afuera y del $3.8 \%$ cuando apunta hacia adentro de la estrella. En el caso de He I $\lambda 5875$, las variaciones son de $2.6 \%$ y $2.1 \%$ respectivamente.

Para complementar los estudios de los perfiles de línea del He en presencia de campos magnéticos, mostramos en la figura 5.9, los perfiles de He I $\lambda 4026, \lambda 4471, \lambda 4921$, y $\lambda 5875$, para modelos correspondientes a una estrella más fría, de tipo espectral B5V, con una temperatura de $15200 \mathrm{~K}$.

Con respecto a los modelos que veníamos estudiando, lo primero que se nota es que las líneas son menos intensas. Además, las variaciones en el centro de la línea son más notorias, por lo menos en las líneas que presentan componentes prohibida, y casi no se advierten variaciones en las alas, lo cual puede deberse a la aproximación utilizada para modelar el efecto Stark. Por otro lado, las componentes prohibidas de He I $\lambda 4026$ y He I $\lambda 4471$ casi no se ven al estar solapadas por sus componentes permitidas. 

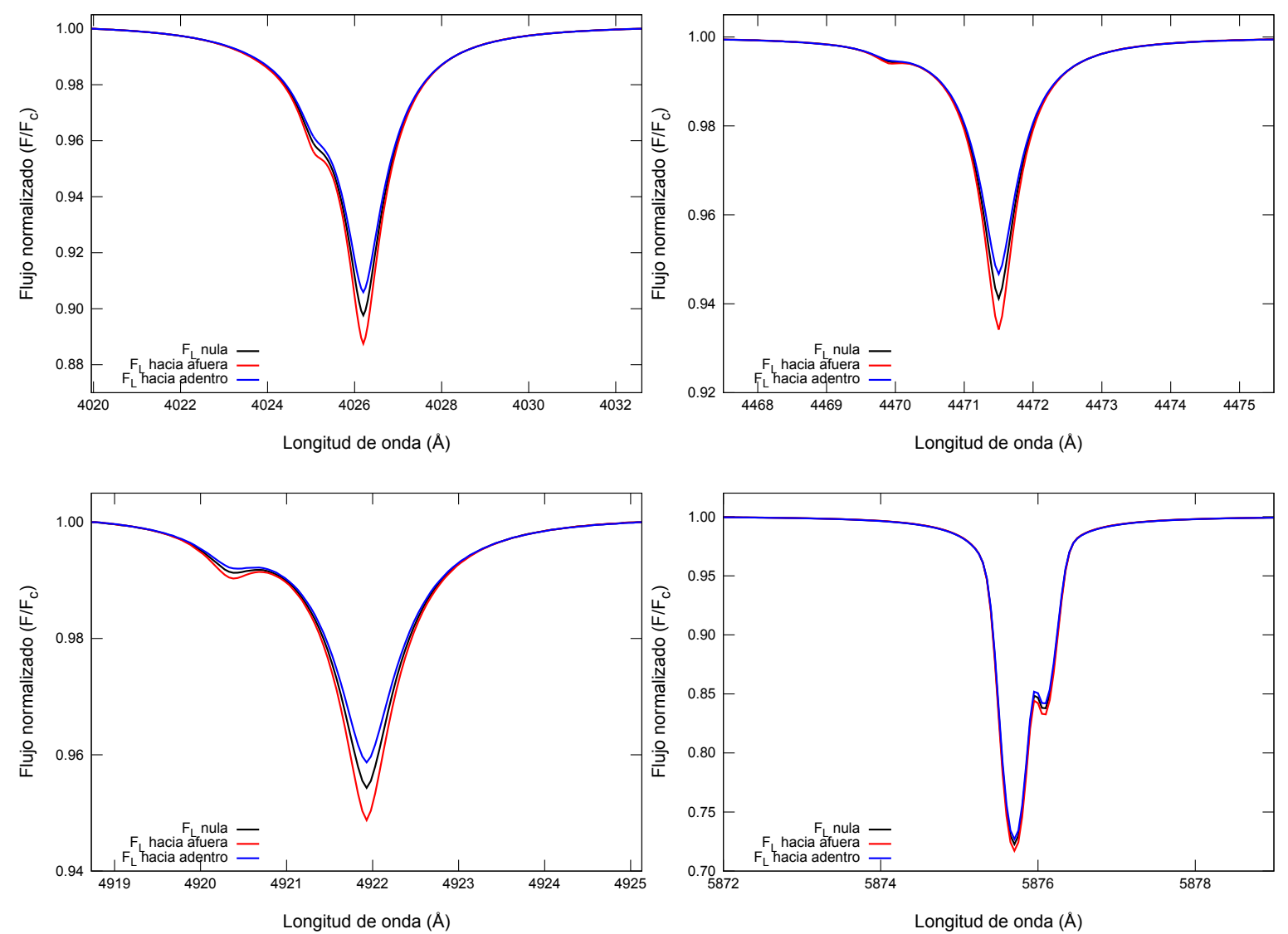

Figura 5.9: Perfiles de línea de He I $\lambda 4026$ (arriba, izquierda), He I $\lambda 4471$ (arriba, derecha), He I $\lambda 4921$ (abajo, izquierda) y He I $\lambda 5875$ (abajo, derecha) para una estrella B5V, con un campo magnético de 1000 Gauss, con $F_{L}$ dirigida al interior (rojo), y hacia el interior (azul). En negro corresponde al modelo sin campo magnético.

En cuanto a los anchos equivalentes, las variaciones en las líneas con componentes prohibidas son mayores que en el caso de las estrellas B2V. Para He I $\lambda 4026$, la variación es de 4.3\% y $5.6 \%$ si la fuerza de Lorentz está dirigida hacia afuera o hacia adentro, respectivamente. Para He I $\lambda 4471$, las variaciones son de $4.6 \%$ y $5.1 \%$, respectivamente, mientras que para el caso de He I $\lambda 4921$, las variaciones llegan a ser de $6.0 \%$ y $7.0 \%$ respectivamente. En cuanto a las líneas de He I $\lambda 5015$ y He I $\lambda 5875$, las variaciones son bastante pequeñas.

\subsection{Efectos locales sobre las líneas de helio dependien- do de la latitud magnética}

Como vimos en el capítulo anterior, la gravedad efectiva depende de la latitud magnética. También pudimos observar que los efectos de los campos magnéticos sobre la gravedad efectiva son máximos en el ecuador magnético, mientras que en los polos magnéticos, el comportamiento es como si el campo magnético fuera nulo. Debido a esto, si consideramos 
un campo magnético cuya intensidad es de 1000 Gauss, al momento de calcular las líneas del He, en el ecuador magnético y en el polo magnético, obtendremos los mismos gráficos que las figuras 5.2, 5.3, 5.4, 5.5 y 5.6, ya que en esa oportunidad, calculamos los modelos en el ecuador magnético, mientras que el perfil de línea obtenido en ausencia de campo magnético coincidirá con el modelo calculado en el polo magnético.

En cuanto a la variación de los anchos equivalentes con la latitud, nuestros resultados para las distintas líneas de He son mostrados en las tablas 5.3 y 5.4

Tabla 5.3: Anchos equivalentes $(\AA)$ de distintas líneas de He I, para diferentes latitudes magnéticas, con la fuerza de Lorentz dirigida hacia el exterior.

\begin{tabular}{|l|l|l|l|l|l||}
\hline Latitud & He I $\lambda 4026.20$ & He I $\lambda 4471.50$ & He I $\lambda 4921.93$ & He I $\lambda 5015.68$ & He I $\lambda 5875.64$ \\
\hline \hline $0^{\circ}$ & 0.5451 & 0.2111 & 0.2762 & 0.4589 & 0.4793 \\
\hline $9^{\circ}$ & 0.5431 & 0.2099 & 0.2756 & 0.4540 & 0.4764 \\
\hline $18^{\circ}$ & 0.5391 & 0.2079 & 0.2747 & 0.4459 & 0.4721 \\
\hline $27^{\circ}$ & 0.5348 & 0.2059 & 0.2738 & 0.4385 & 0.4682 \\
\hline $36^{\circ}$ & 0.5311 & 0.2041 & 0.2731 & 0.4326 & 0.4650 \\
\hline $45^{\circ}$ & 0.5279 & 0.2024 & 0.2726 & 0.4280 & 0.4628 \\
\hline $54^{\circ}$ & 0.5255 & 0.2013 & 0.2721 & 0.4244 & 0.4612 \\
\hline $63^{\circ}$ & 0.5236 & 0.2006 & 0.2715 & 0.4218 & 0.4599 \\
\hline $72^{\circ}$ & 0.5221 & 0.1997 & 0.2712 & 0.4196 & 0.4585 \\
\hline $81^{\circ}$ & 0.5212 & 0.1992 & 0.2710 & 0.4183 & 0.4580 \\
\hline $90^{\circ}$ & 0.5208 & 0.1992 & 0.2708 & 0.4179 & 0.4575 \\
\hline
\end{tabular}

Tabla 5.4: Anchos equivalentes $(\AA)$ de distintas líneas de He I, para diferentes latitudes magnéticas, con la fuerza de Lorentz dirigida hacia el interior.

\begin{tabular}{||l|l|l|l|l|l||}
\hline Latitud & He I $\lambda 4026.20$ & He I $\lambda 4471.50$ & He I $\lambda 4921.93$ & He I $\lambda 5015.68$ & He I $\lambda 5875.64$ \\
\hline $0^{\circ}$ & 0.5451 & 0.2111 & 0.2762 & 0.4589 & 0.4793 \\
\hline $9^{\circ}$ & 0.5469 & 0.2117 & 0.2764 & 0.4634 & 0.4819 \\
\hline $18^{\circ}$ & 0.5493 & 0.2133 & 0.2768 & 0.4699 & 0.4856 \\
\hline $27^{\circ}$ & 0.5512 & 0.2143 & 0.2770 & 0.4750 & 0.4881 \\
\hline $36^{\circ}$ & 0.5527 & 0.2150 & 0.2771 & 0.4788 & 0.4904 \\
\hline $45^{\circ}$ & 0.5537 & 0.2157 & 0.2769 & 0.4821 & 0.4918 \\
\hline $54^{\circ}$ & 0.5544 & 0.2163 & 0.2769 & 0.4841 & 0.4929 \\
\hline $63^{\circ}$ & 0.5550 & 0.2164 & 0.2769 & 0.4859 & 0.4940 \\
\hline $72^{\circ}$ & 0.5553 & 0.2168 & 0.2768 & 0.4870 & 0.4944 \\
\hline $81^{\circ}$ & 0.5554 & 0.2167 & 0.2768 & 0.4878 & 0.4946 \\
\hline $90^{\circ}$ & 0.5555 & 0.2168 & 0.2767 & 0.4879 & 0.4948 \\
\hline
\end{tabular}

A modo de ejemplo de la variación de los anchos equivalentes con la latitud magnética, y dado que el comportamiento es similar para todas las líneas de He, sólo mostraremos en la figura 5.10 los anchos equivalentes correspondientes a las líneas de He I $\lambda 4026$ y de He I $\lambda 5015$. 

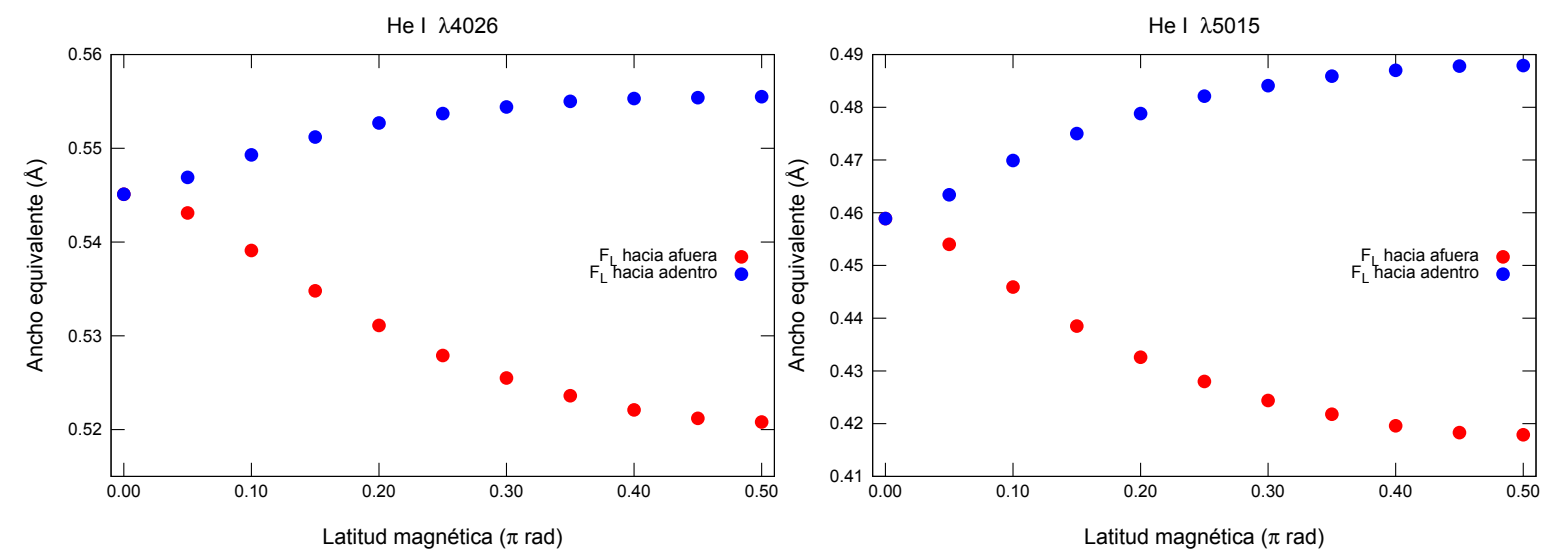

Figura 5.10: Anchos equivalentes de las líneas He I $\lambda 4026$ (izquierda), He I y $\lambda 5015$ (derecha) para distintas latitudes magnéticas, para un campo magnético de 1000 Gauss. Los puntos azules corresponden a modelos con la $F_{L}$ dirigida al interior, mientras que los rojos corresponde al caso de la $F_{L}$ dirigida hacia exterior.

Se puede apreciar que cuando la fuerza de Lorentz apunta hacia el exterior, las variaciones del los anchos equivalentes son mayores. En los casos de la figura 5.10, para la línea He I $\lambda 4026$, la variación del ancho equivalente entre el polo y el ecuador llega a un 4.5\%, mientras que para He I y $\lambda 5015$ llega a un $8.9 \%$.

\subsection{Efectos locales sobre las magnitudes y los índices de color}

La tabla 5.5 muestra las magnitudes visuales absolutas y los índices de color obtenidos para diferentes intensidades del campo magnético $B_{e q}$. Las variaciones locales en el nivel de flujo del continuo originadas por fuerzas dirigidas hacia adentro y hacia afuera son muy pequeñas $(\sim 0.005 \mathrm{mag})$. Esto significa que la fuerza de Lorentz tiene una mayor influencia en las propiedades físicas de las capas superiores de la fotosfera donde se forman principalmente las líneas.

Tabla 5.5: Magnitud visual absoluta e índices de color calculados para una estrella B2V con 22000 $\mathrm{K}$ y $\log g=4$ y diferentes intensidades de $B_{e q}$.

\begin{tabular}{||l||l|l|l||l|l|l||}
\hline \multicolumn{4}{|c|}{} & \multicolumn{4}{|l|}{ Fuerza de Lorentz hacia afuera } & \multicolumn{3}{|l|}{ Fuerza de Lorentz hacia adentro } \\
\hline$B_{e q}$ & $M_{v}$ & $B-V$ & $U-B$ & $M_{v}$ & $B-V$ & $U-B$ \\
\hline \hline $1000 \mathrm{G}$ & -2.414 & -0.230 & -0.912 & -2.409 & -0.228 & -0.907 \\
\hline $500 \mathrm{G}$ & -2.415 & -0.230 & -0.913 & -2.409 & -0.228 & -0.907 \\
\hline $100 \mathrm{G}$ & -2.413 & -0.229 & -0.913 & -2.410 & -0.228 & -0.907 \\
\hline $50 \mathrm{G}$ & -2.412 & -0.229 & -0.912 & -2.411 & -0.229 & -0.908 \\
\hline $0 \mathrm{G}$ & -2.411 & -0.229 & -0.909 & -2.411 & -0.229 & -0.909 \\
\hline
\end{tabular}


En la tabla 5.6 mostramos la magnitud visual absoluta y los índices de color $B-V$ y $U-B$ para un modelo con una intensidad del campo magnético de 1000 Gauss, para las dos direcciones de la fuerza de Lorentz, a distintas latitudes magnéticas, desde el polo magnético $\left(0^{\circ}\right)$ hasta el ecuador magnético $\left(90^{\circ}\right)$. Se puede apreciar a primera vista que las variaciones entre los valores del polo y los del ecuador son siempre mayores cuando la fuerza de Lorentz apunta hacia el interior. Las variaciones en los índices de color son pequeñas, no llegando a más de 3 o 4 milésimas de magnitud entre el polo y el ecuador. En el caso de la magnitud visual, cuando la fuerza de Lorentz apunta hacia el exterior, la diferencia es de 4 milésimas de magnitud, llegando a ser de hasta 9 milésimas de magnitud cuando la fuerza de Lorentz apunta hacia el interior.

Tabla 5.6: Magnitud visual absoluta e índices de color calculados para una estrella B2V con 22000 $\mathrm{K}$ y $\log g=4$ y para diferentes latitudes.

\begin{tabular}{||l||l|l|l||l|l|l||}
\hline \multicolumn{3}{|l|}{} & \multicolumn{3}{|l|}{ Fuerza de Lorentz hacia afuera } & \multicolumn{3}{|l|}{ Fuerza de Lorentz hacia adentro } \\
\hline Latitud & $M_{v}$ & $B-V$ & $U-B$ & $M_{v}$ & $B-V$ & $U-B$ \\
\hline \hline $0^{\circ}$ & -2.411 & -0.217 & -0.885 & -2.411 & -0.217 & -0.885 \\
\hline $18^{\circ}$ & -2.410 & -0.216 & -0.884 & -2.412 & -0.218 & -0.885 \\
\hline $36^{\circ}$ & -2.408 & -0.215 & -0.884 & -2.415 & -0.219 & -0.886 \\
\hline $54^{\circ}$ & -2.407 & -0.214 & -0.884 & -2.418 & -0.220 & -0.887 \\
\hline $72^{\circ}$ & -2.407 & -0.214 & -0.884 & -2.420 & -0.221 & -0.888 \\
\hline $90^{\circ}$ & -2.407 & -0.214 & -0.884 & -2.420 & -0.221 & -0.888 \\
\hline
\end{tabular}

En la tabla 5.7 se muestran los valores de la magnitud visual absoluta y de los índices de color $B-V$ y $U-V$ para distintos valores de la abundancia de He. Se puede apreciar que la variación en la magnitud visual absoluta es un orden de magnitud mayor, aumentando al aumentar la cantidad de He, con variaciones de centésimas de magnitud ( 0.05 mag $)$. El mismo comportamiento ocurre con el índice de color $B-V$, observándose variaciones del orden de la centésima de magnitud. El índice $U-V$ aumenta con la abundancia de He, salvo para la abundancia más baja de He, pero lo hace con variaciones del orden de la milésima, variaciones que se encuentran en el límite de detectabilidad.

Tabla 5.7: Magnitud visual absoluta e índices de color calculados para una estrella B2V con 22000 $\mathrm{K}$ y $\log g=4$ y diferentes abundancias de $\operatorname{He}\left(n_{H e} / n_{H}\right)$.

\begin{tabular}{|l|l|l|l|}
\hline$n_{H e} / n_{H}$ & $M_{v}$ & $B-V$ & $U-B$ \\
\hline \hline 0.03 & -2.387 & -0.221 & -0.889 \\
\hline 0.07 & -2.400 & -0.217 & -0.887 \\
\hline 0.11 & -2.413 & -0.213 & -0.888 \\
\hline 0.17 & -2.427 & -0.209 & -0.890 \\
\hline 0.25 & -2.442 & -0.205 & -0.993 \\
\hline
\end{tabular}




\section{Capítulo 6}

\section{Efectos globales y el modelo del rotador oblicuo}

\subsection{Campo magnético integrado}

Como una consecuencia de la rotación estelar y la orientación relativa del dipolo magnético con el eje de rotación, esperamos que tanto la gravedad efectiva $g_{\text {eff }}$ como la fuerza de Lorentz, cambien a través de la superficie estelar modificando la estructura local de la atmósfera y, por lo tanto, la apariencia del espectro de líneas emergente. En una rotación estelar completa debemos observar diferentes aspectos de la magnetósfera de la estrella; la zona ecuatorial, donde la fuerza de Lorentz tiene su máximo valor o la región polar donde la fuerza magnética desaparece.

Así, para calcular la contribución de la fuerza magnética en los perfiles de línea observados es necesario tener en cuenta los efectos del disco promediado. Con este objetivo hemos calculado modelos magnetosféricos en los que se calcula la estructura de densidad considerando las proyecciones de disco integrado de la componente tangencial del campo magnético $\left\langle B_{\theta} \operatorname{sen} \theta\right\rangle$, el módulo de campo $\langle B\rangle$ y su componente longitudinal $\left\langle B_{z}\right\rangle$ para las diferentes fases de la rotación, como se describe en Valyavin et al. 2004, ver $\S 6.2$, ).

$$
\left\langle B_{\theta} \operatorname{sen} \theta\right\rangle=\frac{3}{\pi(3-\mu)} \int_{0}^{2 \pi} d \varphi_{\star} \int_{0}^{\pi / 2} d \theta_{\star} \operatorname{sen} \theta_{\star} \cos \theta_{\star}\left(1-\mu+\mu \cos \theta_{\star}\right) \operatorname{sen} \theta B_{\theta}(\theta)
$$

donde $\theta$ es la latitud magnética, $B_{\theta}$ es la componente latitudinal del campo magnético generado por un dipolo, $\varphi_{\star}$ y $\theta_{\star}$ son las coordenadas esféricas en el sistema que rota con la estrella, y $\mu$ es el coeficiente de oscurecimiento al limbo. 
Para poder calcular estas integrales es necesario pasar las coordenadas angulares magnéticas al mismo sistema que las coordenadas angulares del sistema de referencia solidario a la estrella. Para eso es necesario realizar las rotaciones correspondientes utilizando el ángulo de oblicuidad magnética $\beta$, que es el ángulo de inclinación entre el eje del dipolo magnético y el eje de rotación de la estrella. Finalmente, para determinar el correspondiente valor de $\left\langle B_{\theta} \operatorname{sen} \theta\right\rangle$ para cada fase, es necesario transformar las coordenadas angulares al sistema del observador, realizando las transformaciones de rotación utilizando el correspondiente valor angular de la fase y el ángulo de inclinación $i$, que es el ángulo de inclinación entre el eje de rotación y la dirección al observador.

Para calcular los valores de $\langle B\rangle$ y $\left\langle B_{z}\right\rangle$ se procede de la misma forma, reemplazando $B_{\theta} \operatorname{sen} \theta$ por $B$ y $B_{z}$, es decir,

$$
\begin{aligned}
\langle B\rangle & =\frac{3}{\pi(3-\mu)} \int_{0}^{2 \pi} d \varphi_{\star} \int_{0}^{\pi / 2} d \theta_{\star} \operatorname{sen} \theta_{\star} \cos \theta_{\star}\left(1-\mu+\mu \cos \theta_{\star}\right) B(\theta) \\
\left\langle B_{z}\right\rangle & =\frac{3}{\pi(3-\mu)} \int_{0}^{2 \pi} d \varphi_{\star} \int_{0}^{\pi / 2} d \theta_{\star} \operatorname{sen} \theta_{\star} \cos \theta_{\star}\left(1-\mu+\mu \cos \theta_{\star}\right) B_{z}(\theta)
\end{aligned}
$$

En la figura 6.1 se muestran estos parámetros magnéticos, como función de la fase de rotación, para un campo magnético dipolar $B_{p}=1000$ Gauss, un ángulo de inclinación $i=90^{\circ}$, una oblicuidad magnética $\beta=90^{\circ}$ y un coeficiente de oscurecimiento al limbo $\mu=0.4$. En este caso, la fuerza de Lorentz está dirigida hacia el interior de la estrella (panel izquierdo) y hacia el exterior (panel derecho).
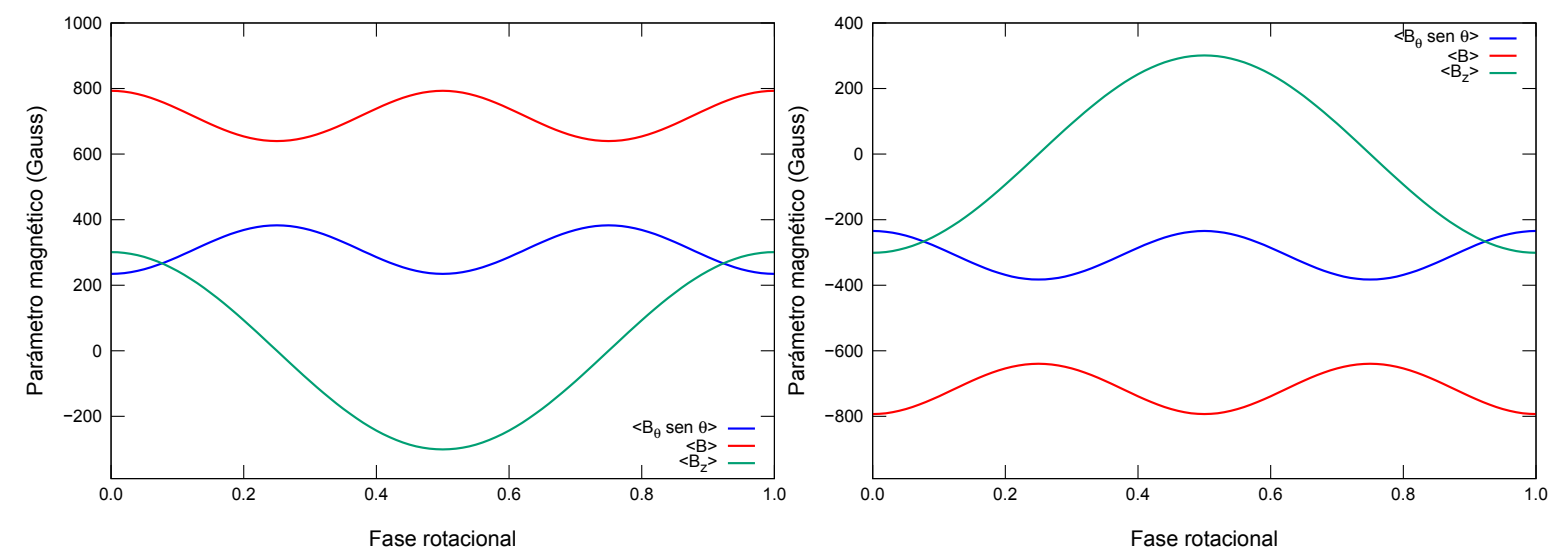

Figura 6.1: Proyecciones de disco integrado de $\left\langle B_{\theta} \operatorname{sen} \theta\right\rangle,\langle B\rangle$ y $\left\langle B_{z}\right\rangle$ en función de la fase rotacional, con $i=90^{\circ}$, y $\beta=90^{\circ}$, para un campo de $1000 \mathrm{G}$, y la fuerza de Lorentz apuntando hacia el interior (izquierda) y exterior (derecha) de la estrella.

Como ejemplo de lo que ocurre para otras intensidades de campos magnéticos, en las figuras 6.2 y 6.3. mostramos los casos correspondientes a campos magnéticos con intensidades de 100 

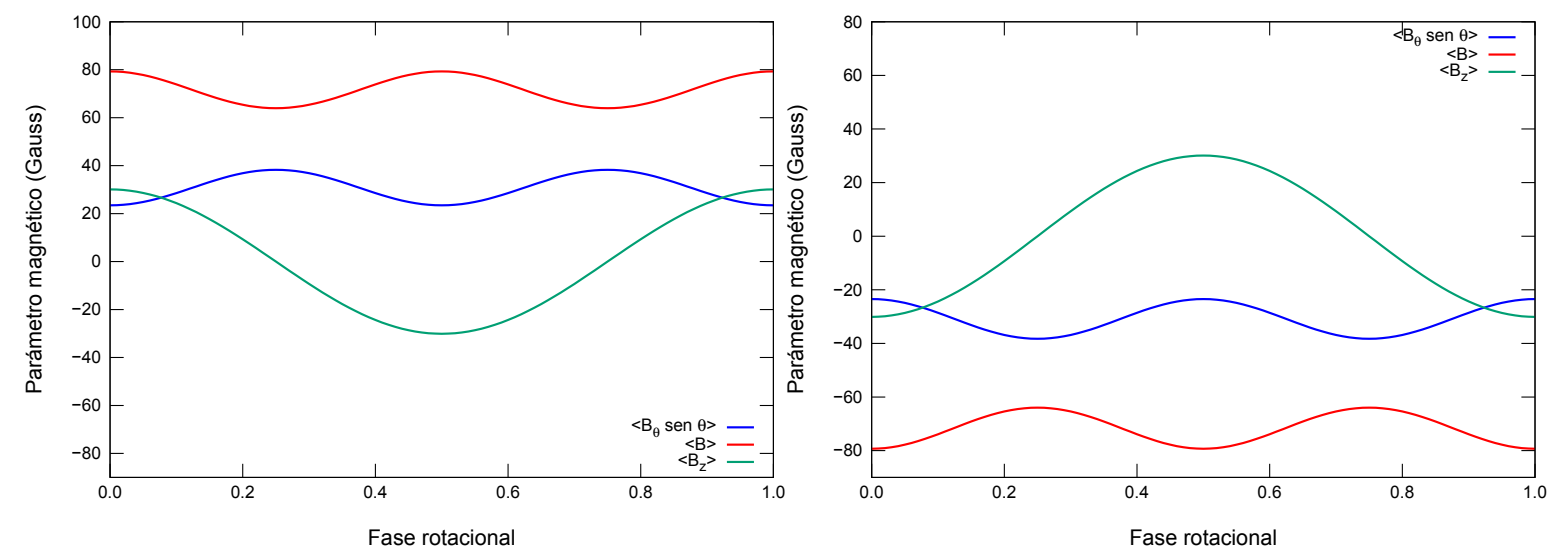

Figura 6.2: Proyecciones de disco integrado de $\left\langle B_{\theta} \operatorname{sen} \theta\right\rangle,\langle B\rangle$ y $\left\langle B_{z}\right\rangle$ en función de la fase rotacional, con $i=90^{\circ}$, y $\beta=90^{\circ}$, para un campo de $1000 \mathrm{G}$, y la fuerza de Lorentz apuntando hacia el interior (izquierda) y exterior (derecha) de la estrella.
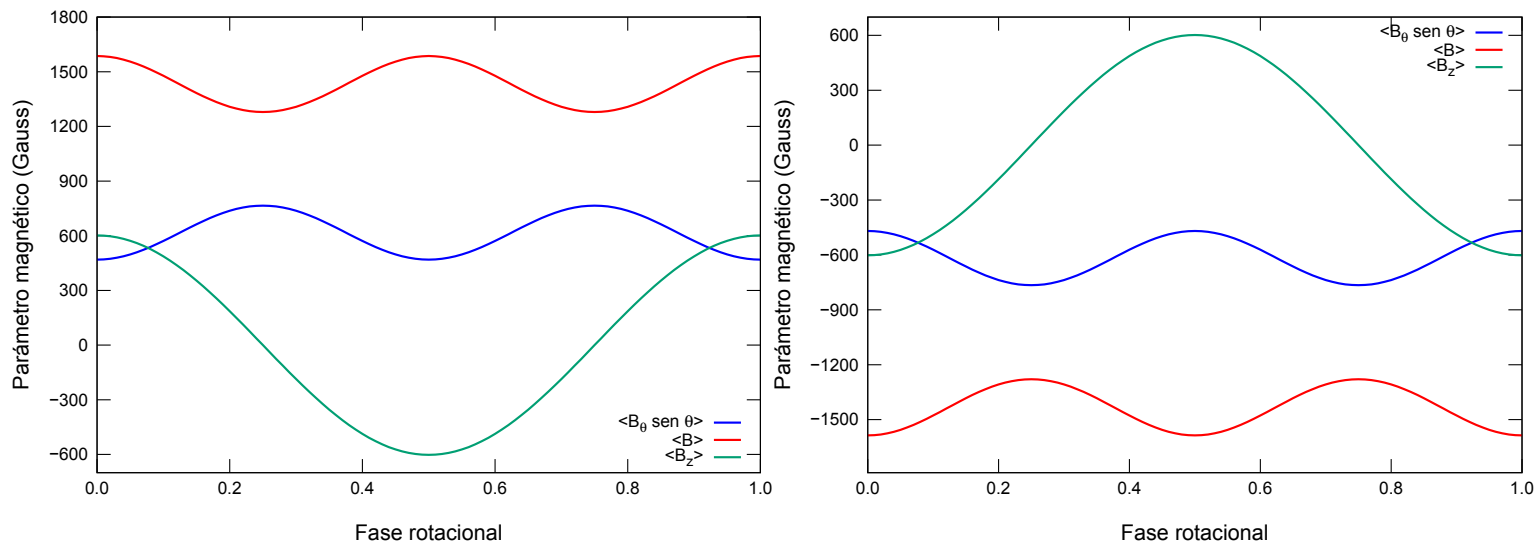

Figura 6.3: Proyecciones de disco integrado de $\left\langle B_{\theta} \operatorname{sen} \theta\right\rangle,\langle B\rangle$ y $\left\langle B_{z}\right\rangle$ en función de la fase rotacional, con $i=90^{\circ}$, y $\beta=90^{\circ}$, para un campo de $2000 \mathrm{G}$, y la fuerza de Lorentz apuntando hacia el interior (izquierda) y exterior (derecha) de la estrella.

y 2000 Gauss, respectivamente. En ambos casos, los gráficos de la izquierda corresponden a la fuerza de Lorentz dirigida hacia el interior, mientras que los de la derecha corresponden a modelos con la fuerza de Lorentz dirigida hacia el exterior.

Cualitativamente, el comportamiento de los parámetros integrados es el mismo, como era de esperarse para las distintas intensidades del campo magnético. Al cambiar la intensidad del campo magnético, lo que cambia son los valores de la amplitud de estos parámetros. En cambio, al invertirse la dirección de la fuerza, se observa el mismo comportamiento pero con el signo cambiado en cada parámetro magnético, es decir, que se invierte la polaridad. 


\subsection{Efectos del campo integrado}

\subsubsection{Líneas espectrales}

Para ver como los campos integrados afectan a los perfiles de línea, en la figura 6.4 mostramos los perfiles de línea de He i $\lambda 5015$ (izquierda) y He i $\lambda 5875$ (derecha). En este caso, la fuerza de Lorentz está apuntando hacia el interior de la estrella. Las hemos graficado para dos fases rotacionales, 0.00 y 0.25 , que es donde toman los valores más extremos como veremos cuando analicemos los anchos equivalenteł Primeramente vemos que los perfiles son menos intensos que los perfiles locales que estudiamos antes. Como se puede apreciar en los gráficos, también vemos que los efectos del campo integrado son muy pequeños comparando cada fase.
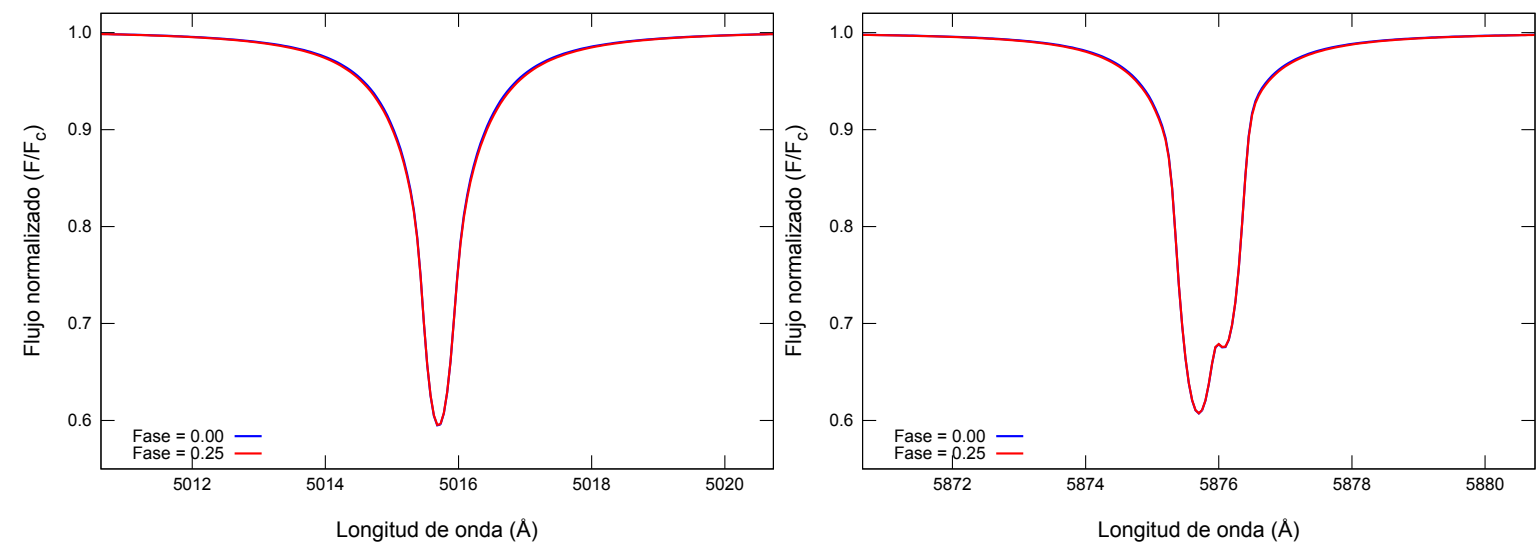

Figura 6.4: Perfiles de línea integrados para las fases rotacionales 0.00 y 0.25 para He I $\lambda 5015$ (izquierda) y He I $\lambda 5875$ (derecha). La fuerza de Lorentz está dirigida hacia el interior y la intensidad del campo es de $1000 \mathrm{G}$
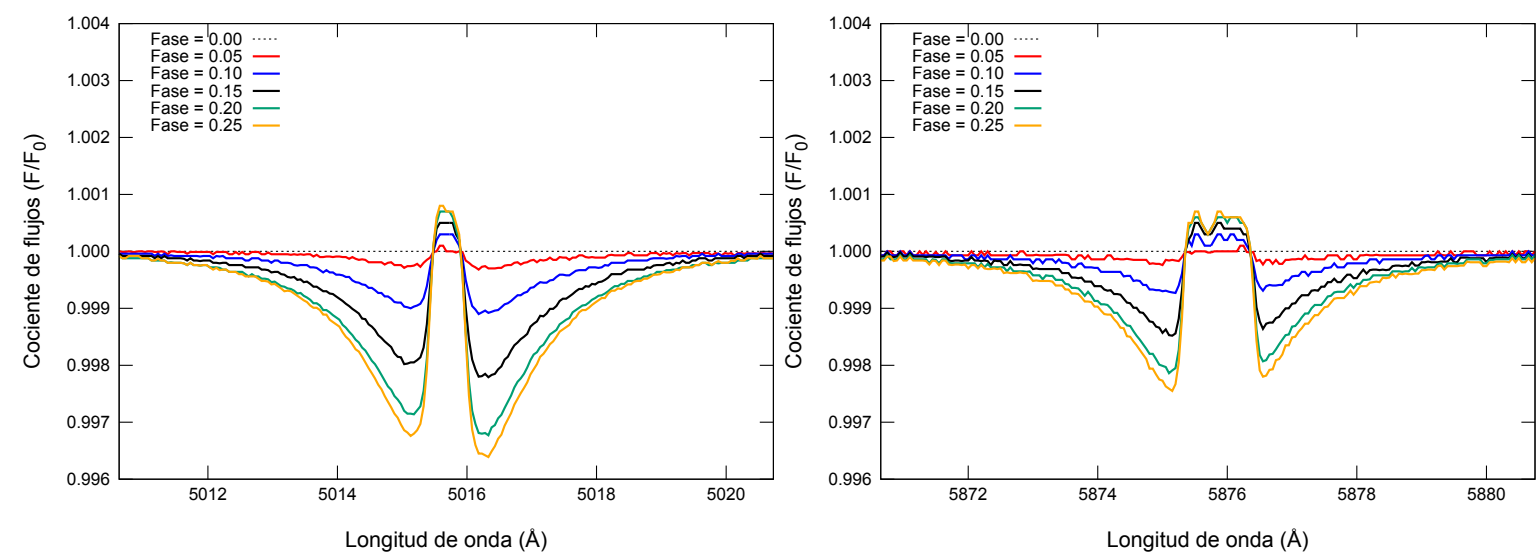

Figura 6.5: Cocientes entre los perfiles de línea en una determinada fase con respecto al perfil de linea en la fase 0. El gráfico izquierdo corresponde a He I $\lambda 5015$ y el derecho a He I $\lambda 5875$

Por este motivo es que presentamos la figura 6.5, en donde se grafica el cociente entre la

\footnotetext{
${ }^{1}$ Los resultados de este capítulo y algunos de los anteriores han sido publicados por Vallverdú et al. 2014 ver referencias).
} 
línea en una determinada fase y la línea en la fase 0.00. Los cocientes que se muestran corresponden a las fases $0.05,0.10,0.15,0.20,0.25$. Los demás perfiles en otras fases se superponen a estos, dado el comportamiento sinusoidal que tienen los perfiles con la fase, que quedará de manifiesto cuando analicemos sus anchos equivalentes. Podemos ver que las variaciones en el centro de las líneas son pequeñas. Las variaciones son mayores en las alas pero siguen siendo pequeñas.

A continuación analizaremos cuál es el comportamiento de los anchos equivalentes en función de la fase rotacional. En particular mostraremos los resultados obtenidos para las líneas He I $\lambda 5015$ y He I $\lambda 5875$ y en la tabla 6.1 listamos los valores de los anchos equivalentes en función de la fase rotacional, para las dos direcciones de la fuerza de Lorentz, hacia el interior (columnas 2 y 3 ) y hacia el exterior (columnas 4 y 5 ).

Tabla 6.1: Anchos equivalentes de las líneas He I $\lambda 5015$ y He I $\lambda 5875$ para distintas fases rotacionales. Las columnas 2 y 3 corresponden a la fuerza de Lorentz dirigida hacia el interior, y las 4 y 5 , dirigida hacia el exterior.

\begin{tabular}{|l||l|l||l|l||}
\hline Fase & He I $\lambda 5015.68$ & He I $\lambda 5875.70$ & He I $\lambda 5015.68$ & He I $\lambda 5875.70$ \\
\hline \hline 0.00 & 0.4826 & 0.5146 & 0.4636 & 0.5027 \\
\hline 0.05 & 0.4835 & 0.5159 & 0.4627 & 0.5019 \\
\hline 0.10 & 0.4856 & 0.5170 & 0.4602 & 0.4997 \\
\hline 0.15 & 0.4886 & 0.5186 & 0.4564 & 0.4968 \\
\hline 0.20 & 0.4914 & 0.5202 & 0.4527 & 0.4956 \\
\hline 0.25 & 0.4922 & 0.5213 & 0.4511 & 0.4946 \\
\hline 0.30 & 0.4914 & 0.5202 & 0.4527 & 0.4956 \\
\hline 0.35 & 0.4886 & 0.5186 & 0.4564 & 0.4968 \\
\hline 0.40 & 0.4856 & 0.5170 & 0.4602 & 0.4997 \\
\hline 0.45 & 0.4835 & 0.5159 & 0.4627 & 0.5019 \\
\hline 0.50 & 0.4826 & 0.5146 & 0.4636 & 0.5027 \\
\hline 0.55 & 0.4835 & 0.5159 & 0.4627 & 0.5019 \\
\hline 0.60 & 0.4856 & 0.5170 & 0.4602 & 0.4997 \\
\hline 0.65 & 0.4886 & 0.5186 & 0.4564 & 0.4968 \\
\hline 0.70 & 0.4914 & 0.5202 & 0.4527 & 0.4956 \\
\hline 0.75 & 0.4922 & 0.5213 & 0.4511 & 0.4946 \\
\hline 0.80 & 0.4914 & 0.5202 & 0.4527 & 0.4956 \\
\hline 0.85 & 0.4886 & 0.5186 & 0.4564 & 0.4968 \\
\hline 0.90 & 0.4856 & 0.5170 & 0.4602 & 0.4997 \\
\hline 0.95 & 0.4835 & 0.5159 & 0.4627 & 0.5019 \\
\hline 1.00 & 0.4826 & 0.5146 & 0.4636 & 0.5027 \\
\hline & & & \\
\hline
\end{tabular}

En los gráficos de la figura 6.6 mostramos la variación de los anchos equivalentes con la fase de rotación de las líneas He i $\lambda 5015$ (en azul) y He i $\lambda 5875$ (en rojo). El gráfico de la izquierda corresponde a modelos con la fuerza de Lorentz dirigida hacia el interior y el de la derecha a modelos con la fuerza de Lorentz apuntando hacia el exterior. Lo primero que 
podemos apreciar es que el comportamiento de la variación de los anchos equivalentes con respecto a su fase rotacional tiene una forma sinusoidal. Además vemos que, si comparamos el comportamiento de una de las líneas respecto de las dos direcciones posibles veremos que se encuentran desfasadas $90^{\circ}$.

Por otro lado, si comparamos los gráficos de la izquierda y derecha de la figura 6.6 con los gráficos de la figura 6.1, respectivamente, vemos que los anchos equivalentes de ambas líneas están en fase con el $\langle B\rangle$, y desfasados $90^{\circ}$ respecto de $\left\langle B_{\theta} \operatorname{sen} \theta\right\rangle$.
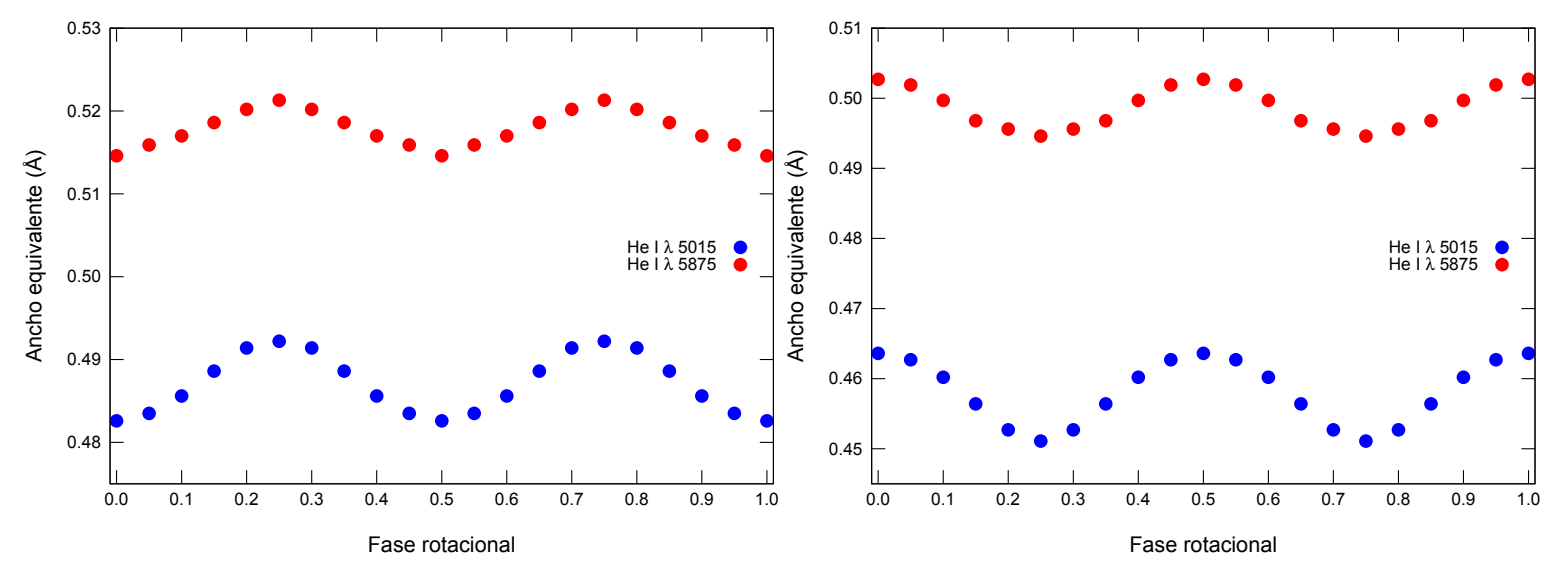

Figura 6.6: Anchos equivalentes en función de la fase rotacional correspondiente a un dipolo de 1000 G de intensidad con la fuerza de Lorentz apuntando hacia el interior (izquierda) y exterior (derecha) de la estrella. Los puntos azules corresponden a He I $\lambda 5015$ y los rojos a He I $\lambda 5875$.

En cuanto a la amplitud de la variación del ancho equivalente, encontramos que para el caso en que la fuerza de Lorentz apunta hacia el interior, la variación es de casi un $2.0 \%$ para el caso de He I $\lambda 5015$ y de $1.3 \%$ en el caso de He I $\lambda 5875$, mientras que si la fuerza de Lorentz apunta hacia el exterior, las variaciones son levemente mayores, $2.8 \%$ y $1.6 \%$ respectivamente.

\subsubsection{Magnitudes e índices de color}

En cuanto a los efectos globales de la presencia de un campo magnético sobre la magnitud absoluta y los índices de color, encontramos que las variaciones con la fase rotacional son bastante pequeñas, a lo sumo un milésimo de magnitud. Estos valores son más pequeños que los valores encontrados localmente que presentamos en la sección 5.5 


\section{Capítulo 7}

\section{Discusión}

Hasta el momento hemos comprobado que la presencia de los campos magnéticos afecta moderadamente a la estructura de la atmósfera. Vimos que las propiedades físicas de la atmósfera no sólo dependen de la intensidad del campo magnético, sino que también dependen de la dirección y del sentido de la fuerza de Lorentz. Todos estos cambios se ven reflejados en la intensidad y forma de las líneas espectrales.

Quizás, el resultado más importante sea la dependencia de las variaciones de densidad y presión con la latitud magnética, ya que los efectos de los campos magnéticos son máximos en el ecuador magnético, y nulos en los polos magnéticos. Esto hace que haya zonas de la atmósfera con distintos comportamientos, de forma que se rompe la simetría esférica al no comportarse todas las regiones de igual manera. Este hecho, combinado con el modelo del rotador magnético oblicuo hace que a medida que la estrella rote veamos zonas de la atmósfera que son distintas en su estructura, lo que se traduce en variaciones periódicas de sus líneas espectrales y en particular las del He.

Hemos de mencionar, que las variaciones en los perfiles de las líneas son pequeñas, pero como veremos más adelante, estas variaciones son mayores si consideramos abundancias de He menores a la solar. En nuestros resultados vemos además que las líneas no varían todas de la misma manera. En algunos casos, las variaciones son mayores en algunas líneas que en las otras. Además, las variaciones de las líneas de He aumentan cuando la fuerza de Lorentz apunta hacia el interior estelar. Si bien las variaciones que encontramos en los anchos equivalentes de las líneas son pequeñas, serían suficientes para explicar las variaciones observadas en algunas estrellas, en general pertenecientes al grupo de las He-weak. Un ejemplo sería la estrella HD 92938 que fuera estudiada por Zboril et al. (1997), cuyas variaciones de la línea He I $\lambda 4471$ a lo largo de 4 noches se muestran en la figura 7.1

Por otro lado, encontramos en varios trabajos observaciones que concordarían con los valores 


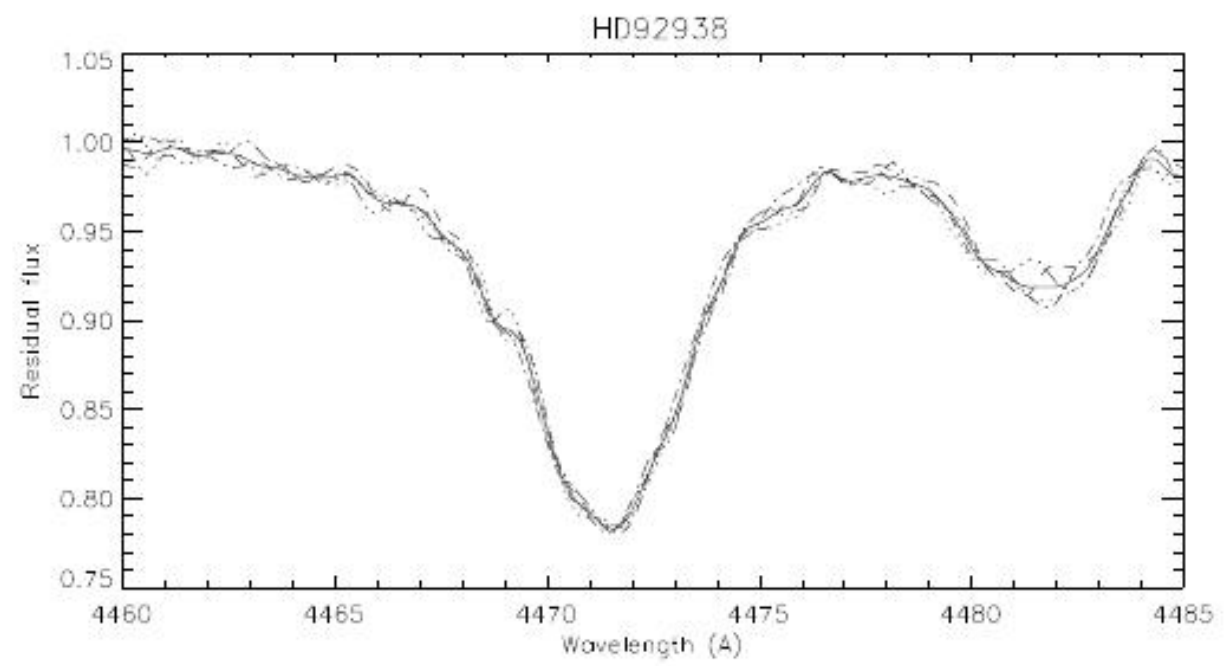

Figura 7.1: Perfil de la línea de He I $\lambda 4471$ de la estrella HD 92938 publicado por Zboril et al. 1997, correspondiente a observaciones de 4 noches.

de los cocientes de intensidades que encontramos para las líneas de He Rivinius et al. 2011. Shultz et al. 2015). A modo de ejemplo en la figura 7.2 mostramos, a la izquierda, el flujo residual de la línea He i $\lambda 4438$ de la estrella HR 2949 Shultz et al. 2015. En ese trabajo, sus autores calculan la diferencia entre la línea observada en distintas fases de rotación con el perfil de línea promedio, mientras que nosotros lo hacemos respecto de la línea calculada sin campo magnético. Se resalta la similitud entre las variaciones calculadas en este trabajo y las observadas (ver figuras (5.2) a (5.6) ) Para las líneas que hemos calculado considerando la abundancia solar, los valores máximos de las variaciones $(I-\langle I\rangle) /\langle I\rangle$ son un poco mayores a 0.01. En cambio, cuando consideramos una abundancia de He menor a la solar, como veremos más adelante en esta sección, la diferencia puede llegar a ser de 0.03, similar al encontrado por Shultz et al. 2015.
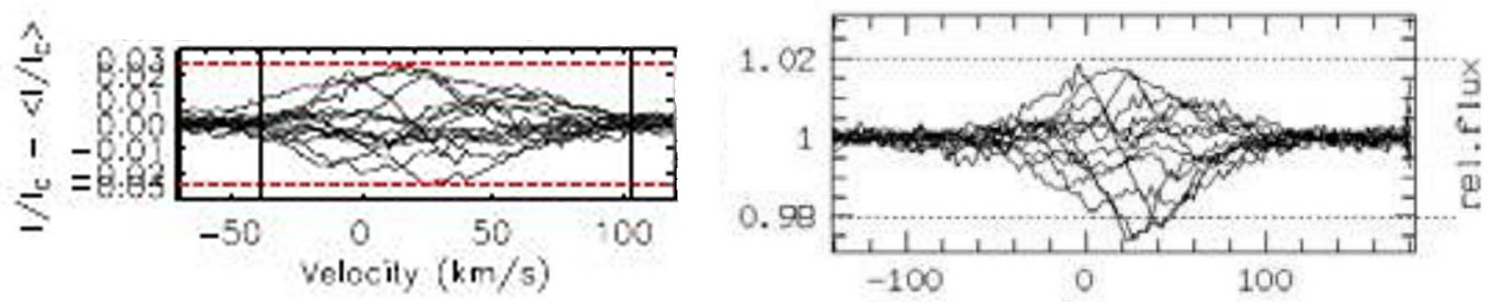

Figura 7.2: Izquierda: Flujo residual de He I $\lambda 4438$ de la estrella HR 2949 Shultz et al. 2015. Derecha: Intensidad relativa de la misma línea Rivinius et al. 2011.

En el lado derecho de la figura 7.2, vemos la intensidad relativa de la misma línea reportada por Rivinius et al. 2011. Nuevamente, el cociente está realizado respecto al valor promedio. Para nuestros modelos, el valor máximo que hemos hallado es de 0.014 respecto del valor de referencia para abundancia solar, mientras que si la abundancia de He es menor, obtenemos 
valores a lo sumo de 0.04 , acorde a lo encontrado en las observaciones

En otros casos encontramos que las variaciones en los anchos equivalentes son mucho mayores que las que hemos encontrado, como lo es el caso particular de a Cen, que es la estrella peculiar de He con variaciones más extremas.

En cuanto a las magnitudes visuales y a los índices de color, los valores que hemos encontrado son un orden de magnitud menor que el típicamente observado. En nuestro caso, encontramos que las variaciones en la magnitud visual absoluta llegan a ser de 9 milésimas de magnitud, mientras que a partir de las observaciones se han reportado variaciones de hasta 5 centésimas de magnitud Adelman y Pyper 1985, Catalano y Leone, 1996, Rivinius et al. 2003.

A continuación ejemplificamos el caso de la estrella a Cen para hacer una discusión sobre las causas que podrían relacionarse con las variaciones extremas que se observan en este objeto.

\subsection{Comparación con las observaciones: las variaciones de a Centauri}

Se ha observado que en algunas estrellas de tipo B, los perfiles de sus líneas, fundamentalmente las del He, muestran variaciones periódicas tanto en intensidad como en su forma. Por otra parte, mencionamos que el período de estas variaciones generalmente coincide con el período de rotación de la estrella. Las variaciones en la intensidad de las líneas espectrales pueden asociarse a variaciones de la abundancia relativa de He en la atmósfera de la estrella. En los casos más extremos, encontramos a HD 125823 (a Cen), en la que podemos ver que por momentos tiene una baja abundancia de He (He-weak) o una alta cantidad de He (Hestrong). Underhill et al. 1975 determinaron que las variaciones de las líneas espectrales de He indicaban un cambio en su abundancia relativa de un $10 \%$ a un $19 \%$ con un período de 8.814 días.

En los gráficos de la figura 7.3 reproducimos, dos figuras del trabajo de Underhill et al. 1975 en donde vemos los perfiles de las líneas de He I $\lambda 4471$ y He I $\lambda 4387$, respectivamente. En ambas figuras se muestran a las líneas en tres fases distintas correspondientes a $J D 1570206$, JD 1572468 y JD 1574880 . Aquí, podemos notar que las variaciones, tanto en intensidad como en la forma de las líneas, son apreciables. En particular, vemos que la profundidad de la línea de He I $\lambda 4387$ varía aproximadamente entre 0.50 y 0.90 , mientras que en el caso de He I $\lambda 4471$ varía entre 0.50 y 0.75 .

Por otro lado, esta estrella, como casi todas las estrellas químicamente peculiares, posee un campo magnético variable que coincide con el período de rotación de la estrella.

En la figura 7.4 mostramos las variaciones del campo magnético en función de la fase uti- 

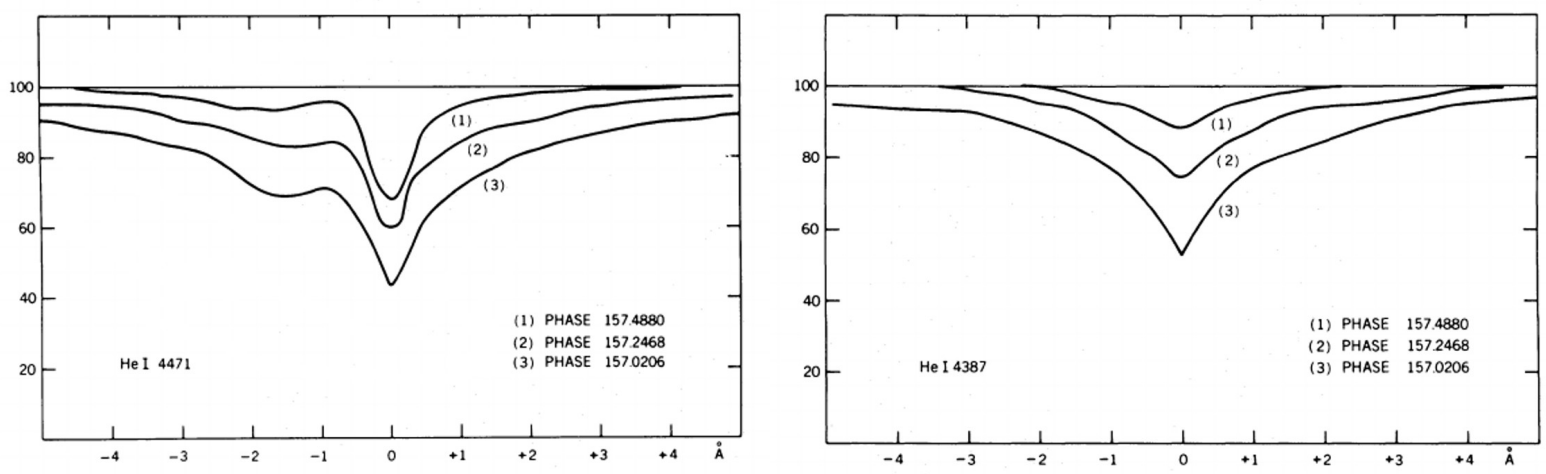

Figura 7.3: Aquí reproducimos dos figuras extraídas del trabajo de Underhill et al. 1975, en donde se muestra, en el gráfico de la izquierda, el perfil de la línea de He I $\lambda 4471$ en tres fases distintas, mientras que en el de la derecha se muestra el perfil correspondiente a la línea de He I $\lambda 4387$.

lizando los datos obtenidos por Wolff y Morrison 1974. Aquí hemos considerando que el período de rotación es de 8.814 días.

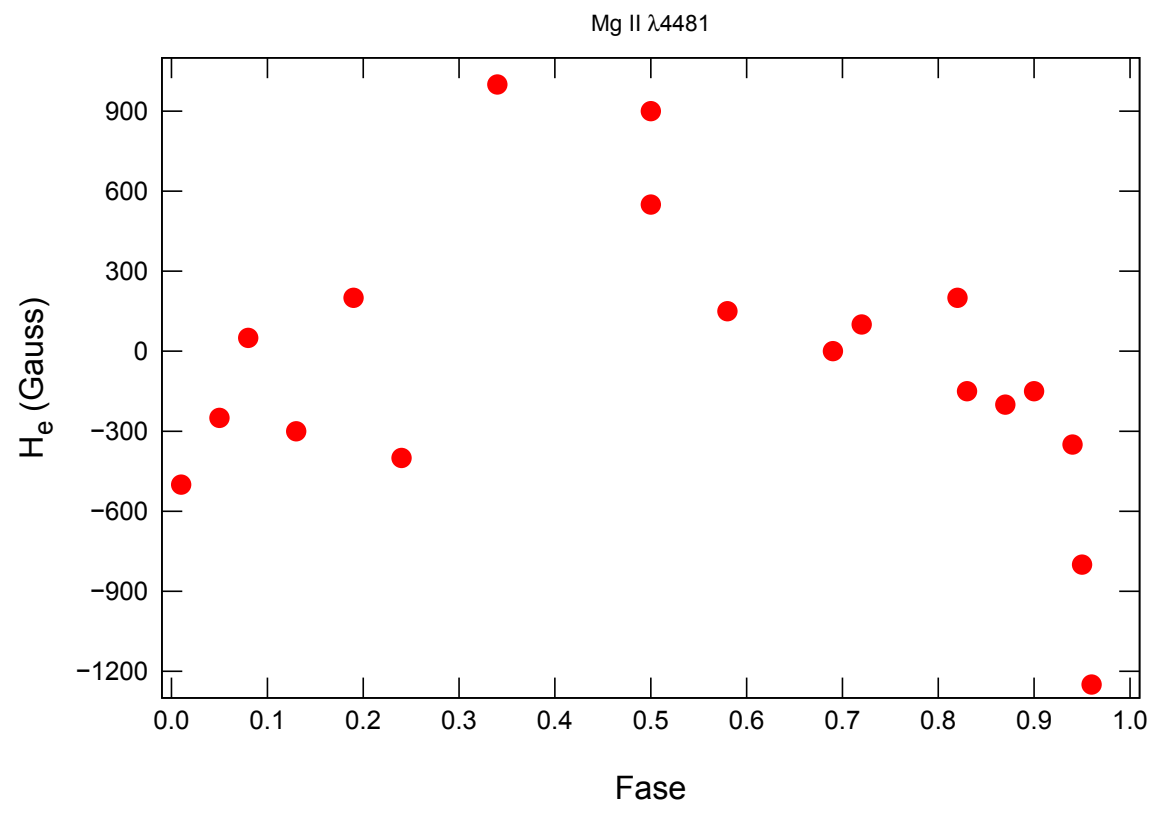

Figura 7.4: Variación de la intensidad del campo magnético de a Cen en función de la fase rotacional.

Las preguntas que nos hacemos son: ¿Existe una variación "real" en la abundancia de He?, y si la respuesta es afirmativa, ¿Cuál es el mecanismo que produce estas variaciones?

Una hipótesis avalada por varios investigadores habla de que densidad de He en la atmósfera estelar no es homogénea, es decir, que existen regiones con una mayor cantidad de He que otras. Al rotar la estrella vemos distintas manchas de He y esto sería la causa de dichas variaciones en los perfiles de línea, y de porqué las variaciones están asociadas al período de rotación. 
A partir de un conjunto de observaciones de a Cen, cedidas gentilmente por Swetlana Hubrig, mostraremos las peculiaridades de esta estrella. Recordemos que a Cen es la que posee variaciones más extremas entre las estrellas peculiares de He.

Las observaciones fueron realizadas en el Observatorio Paranal, Chile, perteneciente a la ESO. Las observaciones consisten de espectros de alta resolución de a Centauri en un rango entre $3020 \AA$ y $10250 \AA$, realizados todas las noches entre el 3 y el 13 de mayo de 2004, con lo cual abarcan un poco más de un período completo de rotación de la estrella.

En la figura 7.5 mostramos cómo variarían en intensidad las líneas de He I $\lambda 4009$, He I $\lambda 4026$, He I $\lambda 4471$, y He I $\lambda 4921$ a lo largo de 10 noches de observación. Se puede ver que las cuatro líneas varían de la misma forma con un período que coincide a primera vista con el período de rotación de la estrella. Todas tienen su máxima intensidad al mismo tiempo y lo mismo ocurre con la intensidad mínima. En particular, Wolff y Morrison 1974 encontraron que la fase del máximo del campo magnético coincide prácticamente con la máxima intensidad de la línea de He i $\lambda 4471$. Así mismo los mínimos del campo coinciden con la menor intensidad de la misma línea.

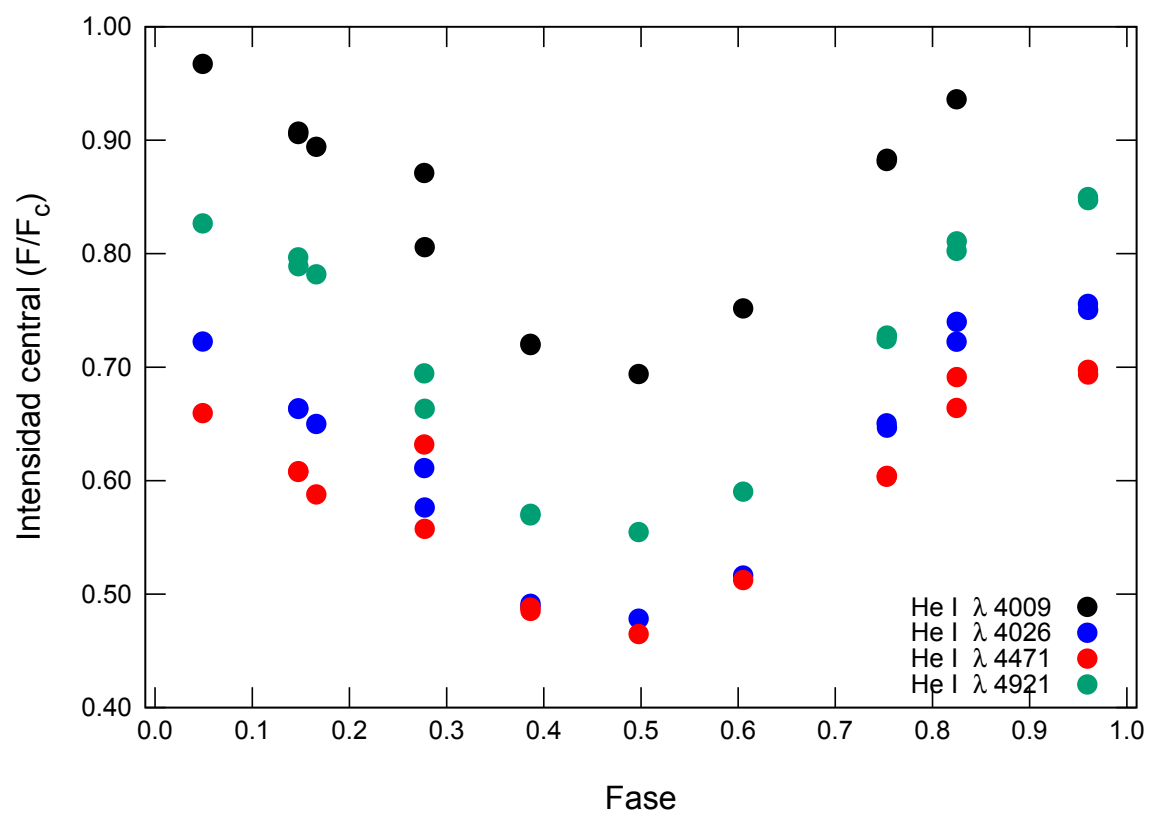

Figura 7.5: Intensidades de las líneas de He I $\lambda 4009, \lambda 4026, \lambda 4471$, y $\lambda 4922$, en función del tiempo a lo largo de las diez noches de observación.

En un principio, extrapolando este comportamiento anómalo de las líneas de He a otros elementos, se podría inferir que las líneas de los demás elementos también cambian de esta manera. Esto sería así, si el efecto observado tiene una característica global que afectase a toda una cara de la estrella. Sin embargo, al observar el comportamiento de la línea de 
Mg II $\lambda 4481$ encontramos otro resultado. Esta línea está muy cerca de la línea de He I $\lambda 4471$ como se muestra en la figura 7.6. De esta forma podemos fácilmente comparar las diferencias de comportamiento de ambas líneas para dos fases de rotación distintas, 0.04 (azul) y 0.27 (rojo). Claramente se puede ver que He I $\lambda 4471$ (a la izquierda) varía notoriamente y que Mg II $\lambda 4481$ (a la derecha) permanece casi invariante.

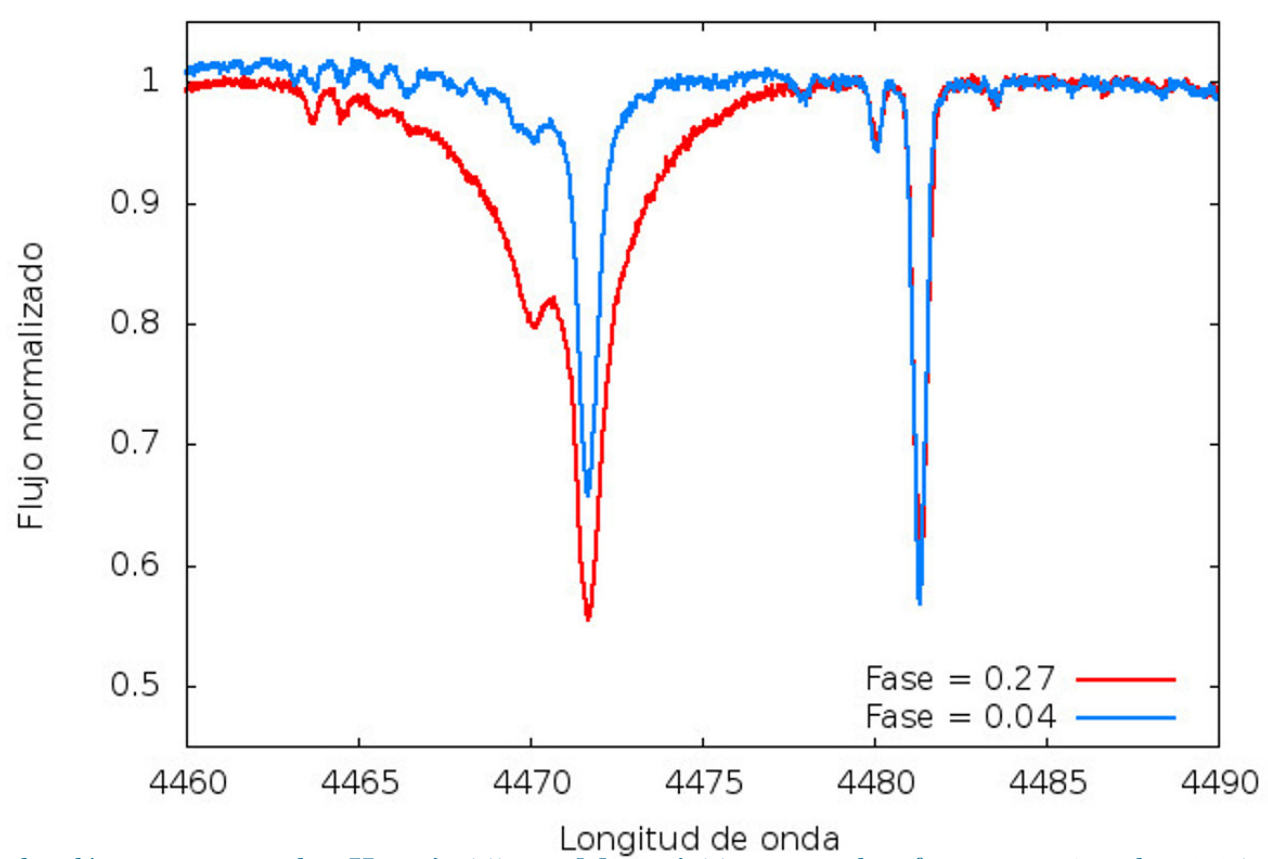

Figura 7.6: las líneas espectrales He I $\lambda 4471$ y Mg II $\lambda 4481$ para las fases rotacionales 0.04 (azul) y 0.27 (roja).

La variación de He I $\lambda 4471$ y la casi invarianza de Mg II $\lambda 4481$ a lo largo de un período de rotación puede observarse claramente en el archivo que se encuentra en http://fcaglp. unlp.edu.ar/ rodolfo/Tesis-Doctoral/Comp-4471-4481.gif. Allí se ve el ciclo completo de variación de ambas líneas.

Este comportamiento puede ilustrarse en la figura 7.7, donde mostramos la variación de la profundidad (intensidad) de la línea en función de la fase de rotación. El gráfico de la izquierda corresponde a He I $\lambda 4471$ y su componente prohibida. Allí podemos observar que la profundidad de la línea varía entre 0.4620 y 0.6983 para la componente permitida, lo cual representa una variación del orden del 33.8\%. En el caso de la componente prohibida, la variación es entre 0.7062 y 0.9606 , correspondiente a una variación del orden del $26.5 \%$. En el gráfico de la derecha mostramos la variación de Mg II $\lambda 4481$ con la fase rotacional. En este caso la variación es entre 0.5392 y 0.6047 , cuya variación porcentual es del orden del 10.8\%. Claramente se ve que, si bien Mg II $\lambda 4481$ varía, lo hace a una escala mucho menor que las componentes de He i $\lambda 4471$. Esto nos indica que el comportamiento anómalo es del He y no de todos los elementos químicos, motivo por el cual a estas estrellas se las conoce 
como químicamente peculiares de He.
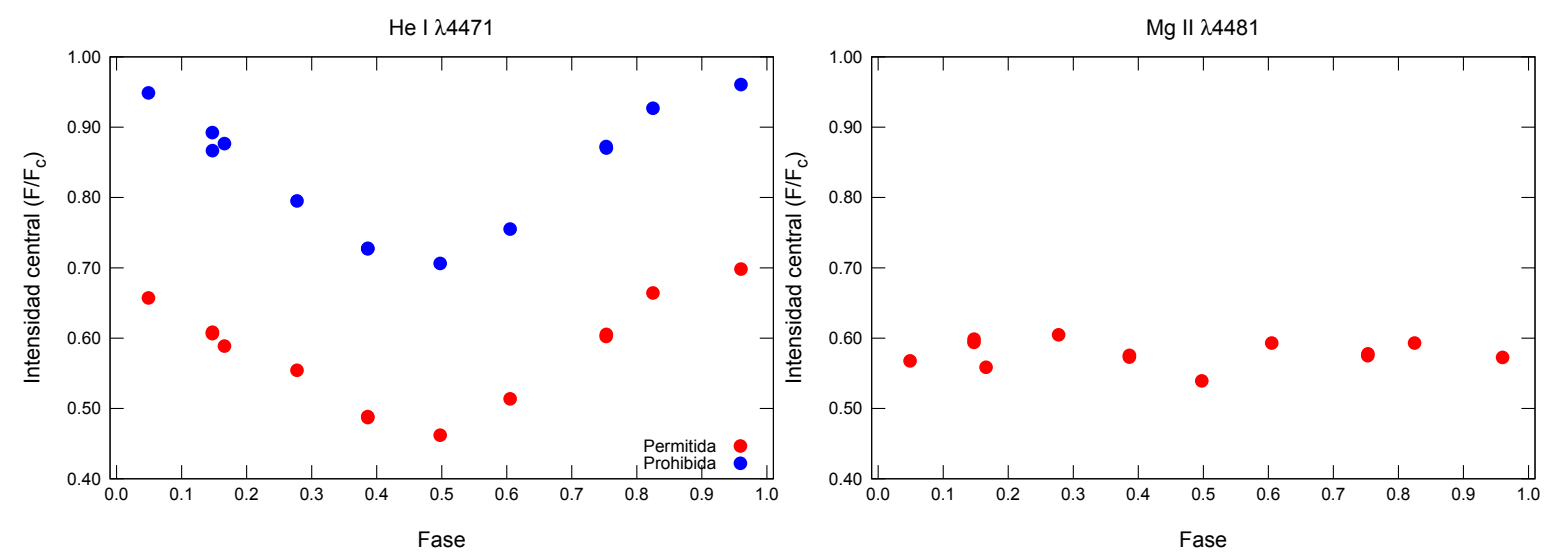

Figura 7.7: A la izquierda, variación de la intensidad (profundidad) de He I $\lambda 4471$ y su componente prohibida con la fase rotacional. A la derecha la correspondiente variación de $\mathrm{Mg}$ II $\lambda 4481$.

En la figura 7.8 mostramos la variación de los anchos equivalentes de las líneas He i $\lambda 4009$, He I $\lambda 4026$, He I $\lambda 4471$ y He I $\lambda 4922$. Vemos que no tienen un comportamiento tan similar entre ellas. La línea de He i $\lambda 4921$ (en verde), muestra un comportamiento sinusoidal claro, tal como las intensidades, coincidiendo el máximo de su ancho equivalente con su mayor intensidad, y la misma coincidencia con los mínimos, correlacionando esta variación con la variación del campo magnético. Podríamos decir lo mismo que la línea He i $\lambda 4009$ (en negro). Distinto es el caso de las líneas de He I $\lambda 4026$ (en azul) y de He I $\lambda 4471$ (en rojo), donde la variación es más suave e irregular.

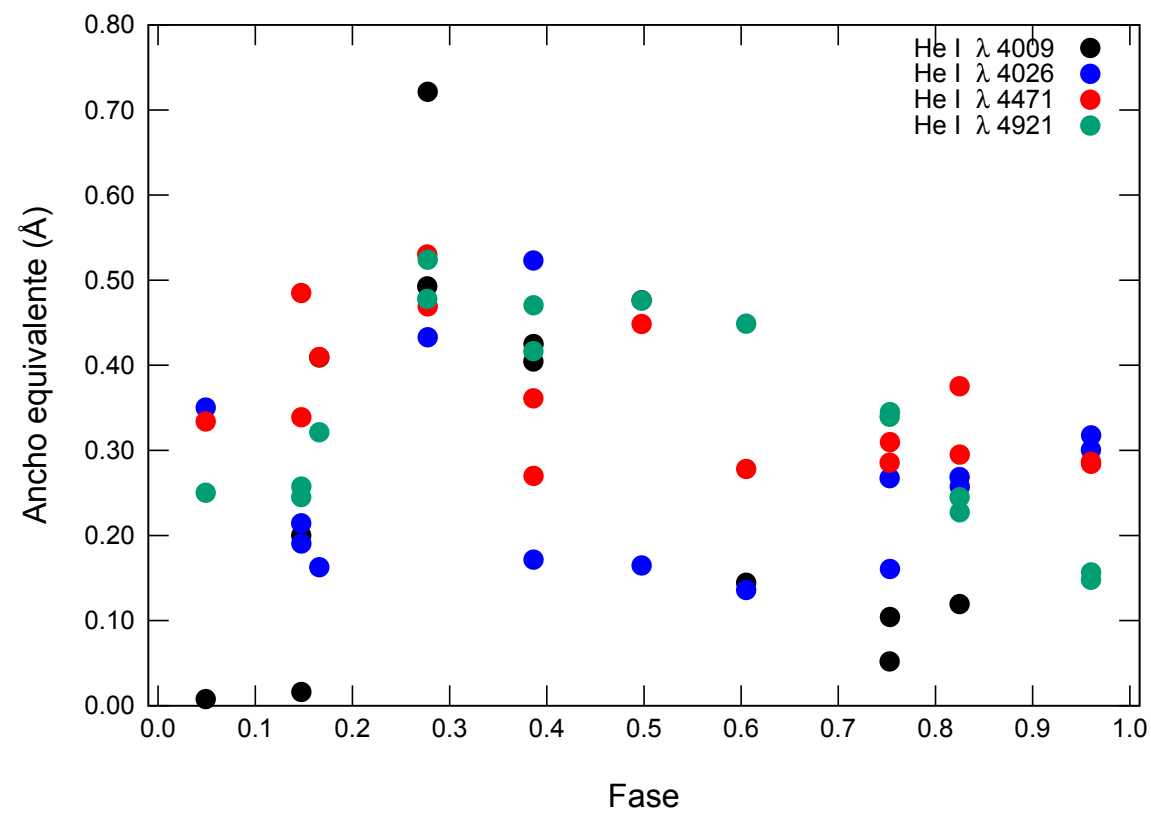

Figura 7.8: Anchos equivalentes de las líneas de He I $\lambda 4009, \lambda 4026, \lambda 4471$, y $\lambda 4922$, en función del tiempo a lo largo de las diez noches de observación. 
Para discutir este comportamiento, en la siguientes secciones analizamos los efectos de la abundancia relativa de He en los perfiles de línea considerando casos en presencia y ausencia de campos magnéticos.

\subsection{Efectos de la composición química del He}

Con los resultados antes expuestos en el capítulo \$5 podemos ver que el solo efecto del campo magnético no es suficiente para describir las variaciones en la intensidad de las líneas de algunas estrellas, en especial las denominadas He-strong.

Es sabido que para cualquier elemento químico, cuando más abundante sea este en la atmósfera, sus correspondientes líneas serán más intensas y más anchas. Esto permite, si medimos el ancho equivalente de las líneas de un dado elemento químico, estimar la abundancia del mencionado elemento, por lo menos en la región de formación de la línea.

Esta relación que existe entre la intensidad de la línea y la abundancia, hace pensar que las variaciones que se observan en las estrellas químicamente peculiares se atribuyan a variaciones en las abundancias químicas. Incluso, se puede determinar cuáles serían dichas abundancias Underhill et al. 1975. Las variaciones temporales de una misma estrella, y su correlación con la rotación de la estrella, indicarían que la distribución química de los distintos elementos no es homogénea, sino que existen regiones con sobreabundancias, y otras regiones con subabundancias químicas. De aquí surgió la hipótesis de que las estrellas químicamente peculiares presentan "manchas" de determinados elementos químicos como hoy en día se demuestra con la técnica de tomografía Doppler.

En las figuras 7.9, 7.10 y 7.11, mostramos el espectro de varias líneas de He calculadas para una estrella con una temperatura efectiva de $22000 \mathrm{~K}$ y distintas abundancias relativas de He $\left(n_{H e} / n_{H}\right): 0.03,0.07,0.11,0.17$, y 0.25 .

Se observa notoriamente las variaciones en la intensidad y en el ancho de la línea. Se ve por ejemplo, que para la línea de He i $\lambda 5015$ el valor de $F(\Delta \lambda=0) / F_{C}$ varía entre 0.58 cuando la abundancia de He es mayor y 0.63 cuando la abundancia es menor. Esto se traduce en que la variación de la intensidad sería del orden de un 8-9\%. Esta variación es un factor 2 o 3 mayor que la obtenida teóricamente al considerar una magnetósfera con abundancia constante como se mostró en el capítulo $\$ 5$ pero continúa siendo inferior a la observada en a Cen. 


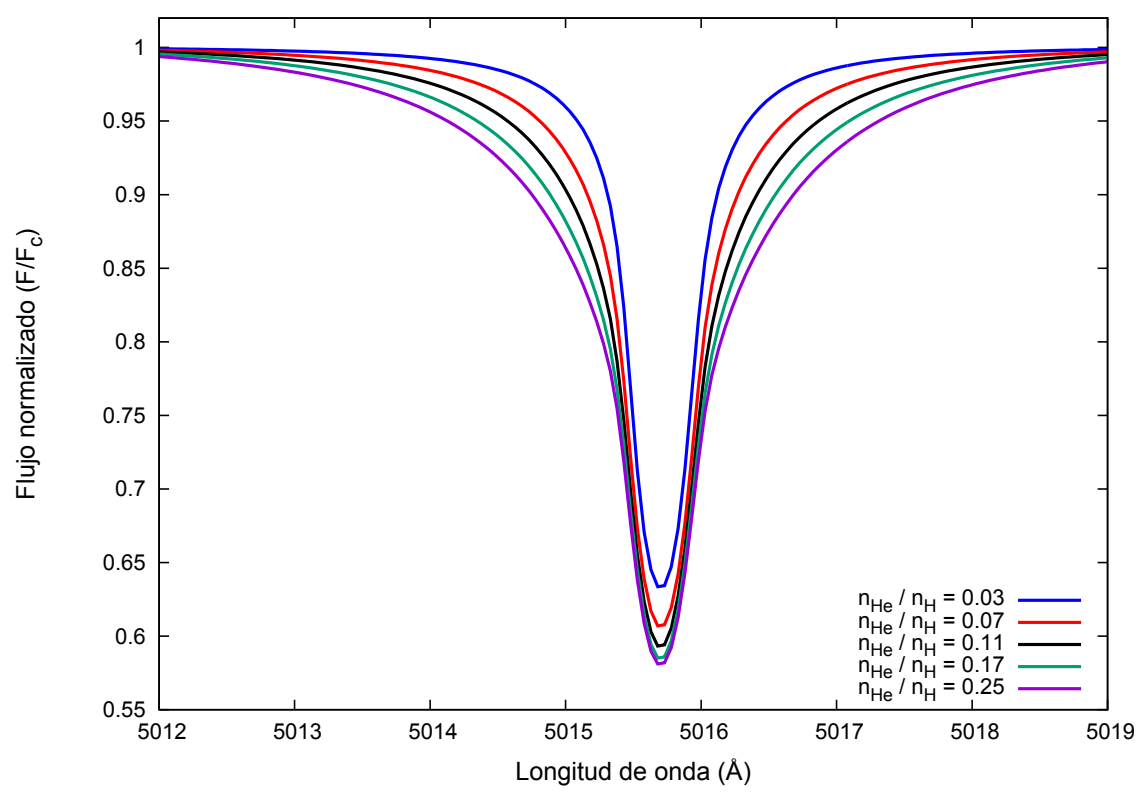

Figura 7.9: Perfiles de la línea de He i $\lambda 5015$ para diferentes abundancias de He en la superficie.
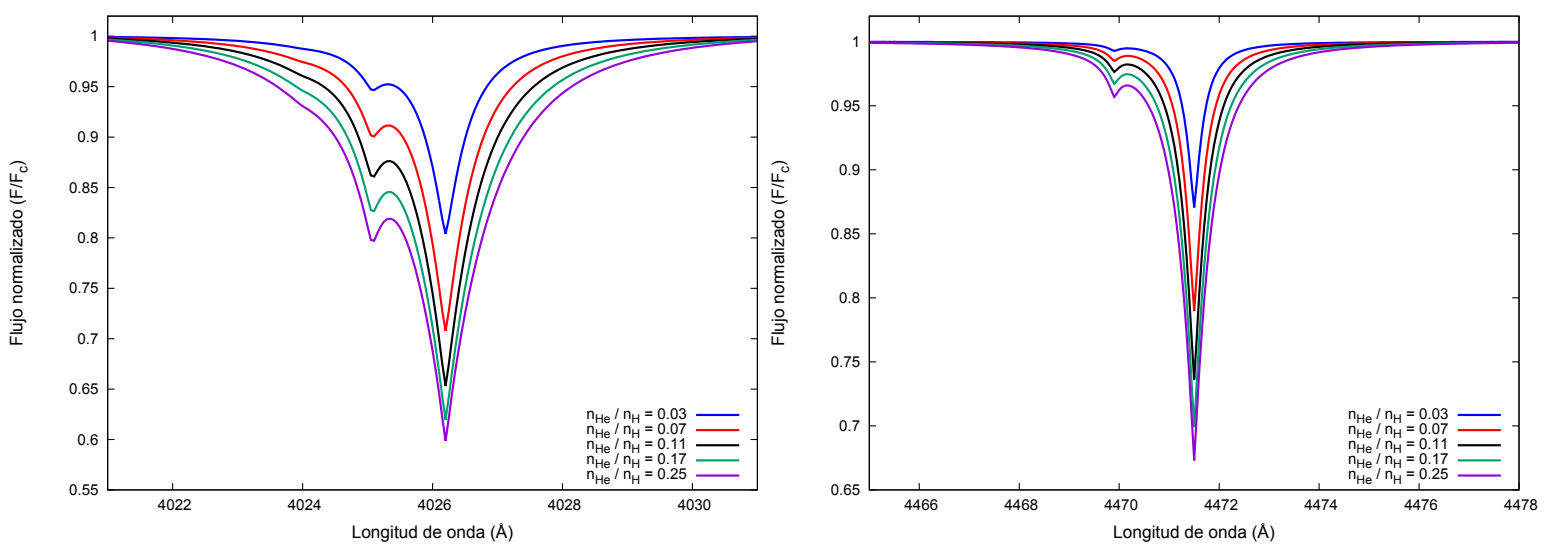

Figura 7.10: A la izquierda se muestra la variación en el perfil de la línea He I $\lambda 4026$ debida a diferentes abundancias de He, mientras que a la derecha se muestran las variaciones en la línea He I $\lambda 4471$.
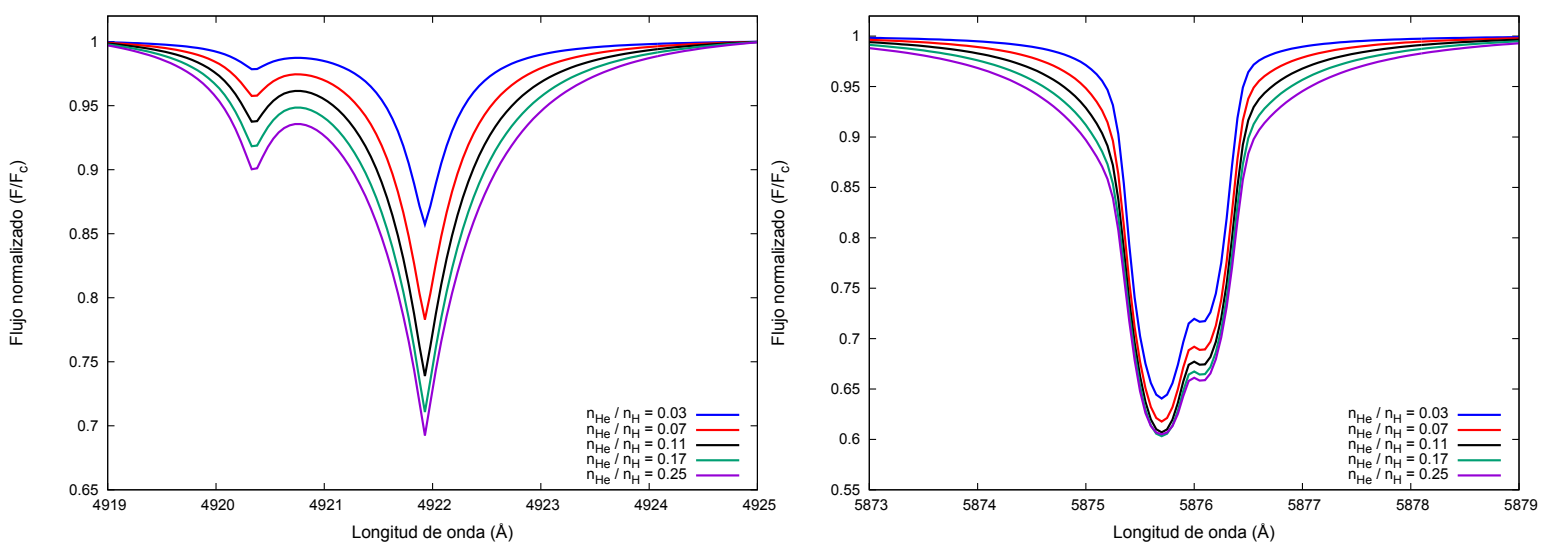

Figura 7.11: A la izquierda mostramos la variación en el perfil de la línea He I $\lambda 4921$ debida a diferentes abundancias de He. A la derecha mostramos las variaciones en la línea de He i $\lambda 5875$. 
Por otra parte, vemos que las variaciones son distintas para cada línea, como se muestra en la tabla 7.1, encontrando que éstas varían un 15-18\% para He I $\lambda 4026$, un 9-10\% para He I $\lambda 4471$, un $19-23 \%$ para He I $\lambda 4921$ y un $6-7 \%$ para el caso de He I $\lambda 5875$.

Tabla 7.1: $F / F_{c}$ del centro de distintas líneas de He I para distintas abundancias de He $\left(n_{H e} / n_{H}\right)$.

\begin{tabular}{|l|l|l|l|l|l|}
\hline Línea & 0.03 & 0.07 & 0.11 & 0.17 & 0.25 \\
\hline \hline He I $\lambda 4026.20$ & 0.80 & 0.71 & 0.65 & 0.62 & 0.60 \\
\hline He I $\lambda 4471.50$ & 0.87 & 0.79 & 0.74 & 0.70 & 0.67 \\
\hline He I $\lambda 4921.93$ & 0.86 & 0.78 & 0.74 & 0.71 & 0.69 \\
\hline He I $\lambda 5015.68$ & 0.63 & 0.61 & 0.59 & 0.59 & 0.58 \\
\hline He I $\lambda 5875.70$ & 0.64 & 0.62 & 0.61 & 0.60 & 0.60 \\
\hline
\end{tabular}

En la tabla 7.2 mostramos los anchos equivalentes de las cinco líneas de He I tratadas anteriormente para las distintas abundancias de He. Claramente se ve la dependencia proporcional del ancho equivalente con la abundancia. Este resultado coincide con lo observado, dado que las líneas tienen origen a distintas profundidades de la atmósfera.

Tabla 7.2: Anchos equivalentes de distintas líneas de He I para distintas abundancias de He $\left(n_{H e} / n_{H}\right)$.

\begin{tabular}{||l|l|l|l|l|l||}
\hline \hline Línea & 0.03 & 0.07 & 0.11 & 0.17 & 0.25 \\
\hline \hline He I $\lambda 4026.20$ & 0.2385 & 0.4207 & 0.5742 & 0.7109 & 0.8371 \\
\hline He I $\lambda 4471.50$ & 0.0839 & 0.1568 & 0.2244 & 0.2896 & 0.3553 \\
\hline He I $\lambda 4921.93$ & 0.1213 & 0.2136 & 0.2906 & 0.3584 & 0.4191 \\
\hline He I $\lambda 5015.68$ & 0.2893 & 0.3924 & 0.4745 & 0.5476 & 0.6156 \\
\hline He I $\lambda 5875.64$ & 0.3497 & 0.4291 & 0.4909 & 0.5453 & 0.5956 \\
\hline \hline
\end{tabular}

\subsubsection{Efectos combinados de la composición química y la presencia de campos magnéticos}

En lo que sigue, discutimos el efecto de distintas abundancias superficiales de $n_{H e} / n_{H}$ sobre las líneas de He. Para ello, utilizando nuestro código de atmósfera, calculamos las líneas de He correspondientes a una estrella con los parámetros de una B2V como en los casos anteriores, pero en este caso la abundancia fraccional de He es 0.20 , es decir $n_{H e} / n_{H}=0.07$. En todos los modelos, la intensidad del campo magnético es de 1000 Gauss.

En la figura 7.12 mostramos el perfil de la línea He I $\lambda 4026$. A diferencia de los perfiles que obtuvimos para los modelos con abundancia solar (ver sección $\$ 5.3$, vemos que las variaciones son mayores. Para el caso en que la fuerza de Lorentz apunta hacia el exterior, vemos que la variación en la intensidad de la componente permitida llega un poco más del $4.0 \%$, y en el caso de la componente prohibida, llega a un 0.7\%. En cambio, para una 

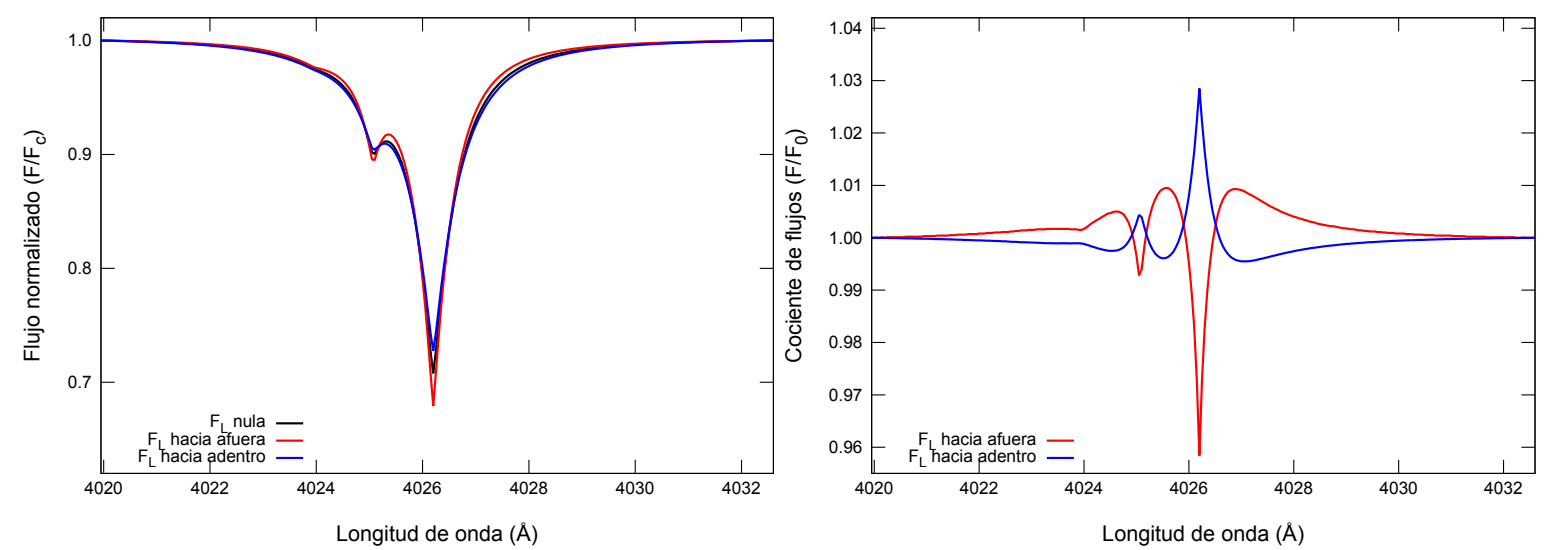

Figura 7.12: Perfil normalizado de la línea He I $\lambda$ 4026. Graficamos el perfil correspondiente a un modelo sin campo magnético (negro), y con un campo de $1000 \mathrm{G}$ con la $F_{L}$ dirigida hacia afuera (rojo), y con $F_{L}$ dirigida hacia adentro (azul). A la derecha se muestra el cociente entre los flujos con y sin campo magnético.
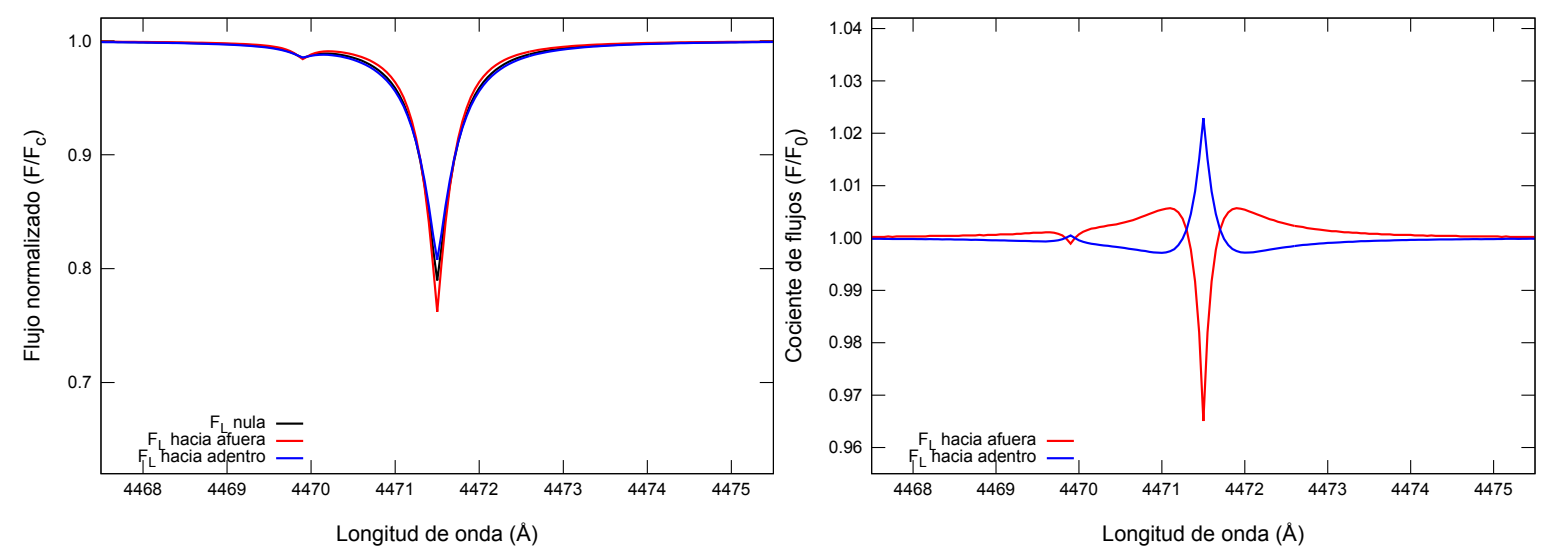

Figura 7.13: Perfil normalizado de la línea He I $\lambda 4471$. Graficamos el perfil correspondiente a un modelo sin campo magnético (negro), y con un campo de $1000 \mathrm{G}$ con la $F_{L}$ dirigida hacia afuera (rojo), y con $F_{L}$ dirigida hacia adentro (azul). A la derecha se muestra el cociente entre los flujos con y sin campo magnético.

fuerza de Lorentz apuntando hacia el interior, las variaciones son de casi un $3.0 \%$ y $0.4 \%$, respectivamente. Considerando las variaciones relativas entre los modelos con la fuerza de Lorentz en direcciones opuestas, tendríamos un 7.0\%, valor consistente con observaciones de estrellas He-weak. En cuanto a las alas, las variaciones llegan a casi el $1 \%$.

En el caso de He I $\lambda 4471$ (figura 7.13), las variaciones son menores, entre un $3.7 \%$ (fuerza de Lorentz hacia afuera) y un 2.5\% (fuerza de Lorentz hacia adentro). Al igual que en los casos anteriores la variación de la intensidad de la componente prohibida es mínima, mucho menor que la variación en las alas de la componente permitida.

En el caso de las líneas He i $\lambda 5015$ (figura 7.14) y He i $\lambda 5875$ (figura 7.15), vemos que casi no hay variaciones en la intensidad del centro de las líneas, mientras que los cambios se observan fundamentalmente en las alas. Allí las variaciones alcanzan un $2 \%$ He i $\lambda 5015$ y aproximadamente un $1.2 \%$ en el caso de He I $\lambda 5875$. 

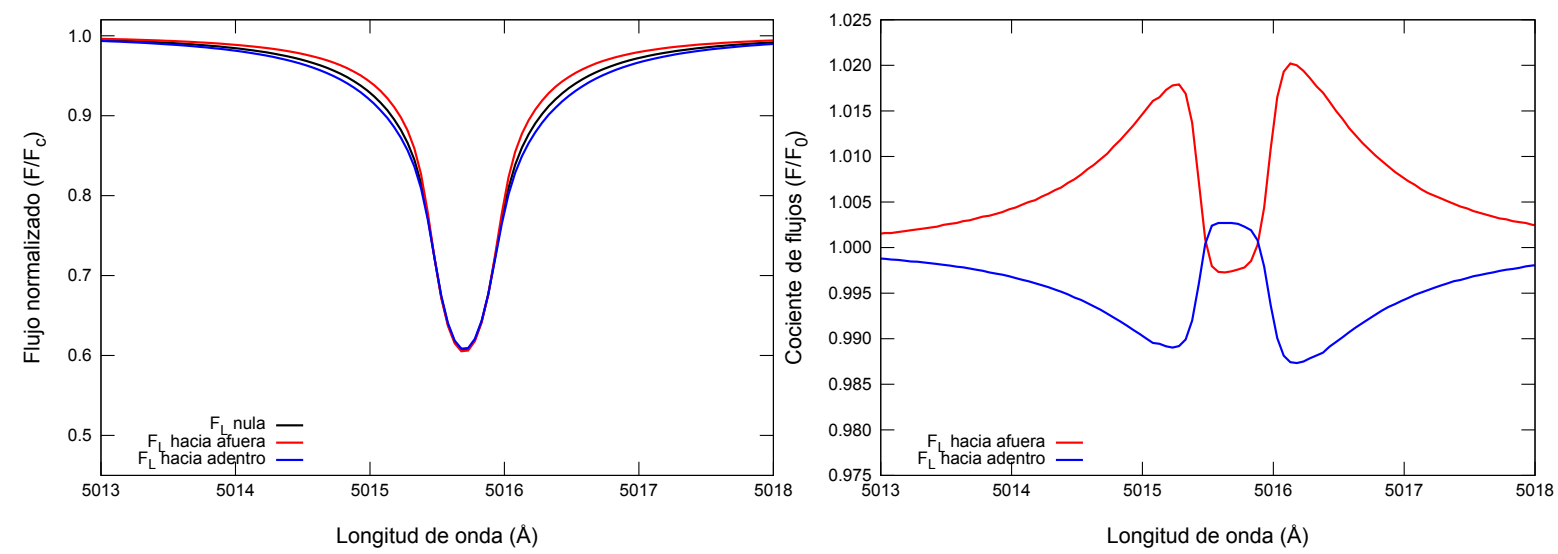

Figura 7.14: Perfil normalizado de la línea He I $\lambda$ 5015. Graficamos el perfil correspondiente a un modelo sin campo magnético (negro), y con un campo de $1000 \mathrm{G}$ con la $F_{L}$ dirigida hacia afuera (rojo), y con $F_{L}$ dirigida hacia adentro (azul). A la derecha se muestra el cociente entre los flujos con y sin campo magnético.
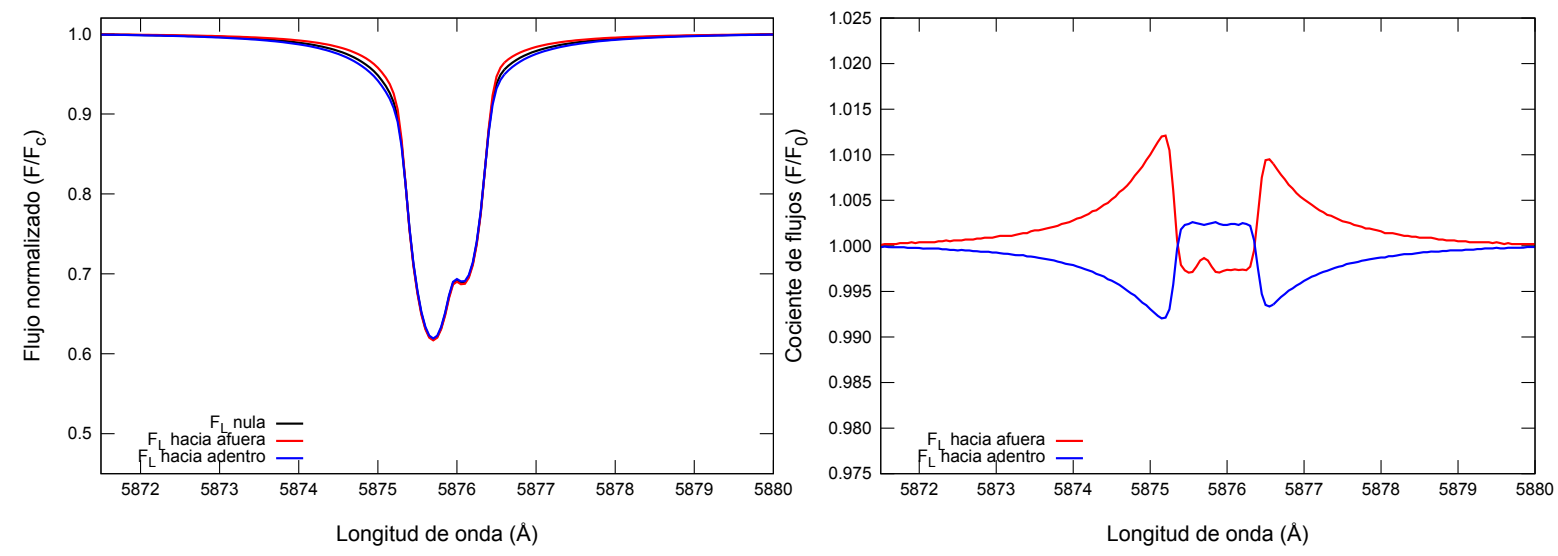

Figura 7.15: Perfil normalizado de la línea He I $\lambda 5875$. Graficamos el perfil correspondiente a un modelo sin campo magnético (negro), y con un campo de $1000 \mathrm{G}$ con la $F_{L}$ dirigida hacia afuera (rojo), y con $F_{L}$ dirigida hacia adentro (azul). A la derecha se muestra el cociente entre los flujos con y sin campo magnético.

En la tabla 7.3. listamos los anchos equivalentes para las líneas de He, para los modelos con distintas intensidades de los campos magnéticos y para las dos direcciones de la fuerza de Lorentz.

Al igual que en el caso de una abundancia solar, encontramos que, si la fuerza de Lorentz está dirigida hacia el exterior de la estrella, los anchos equivalentes decrecen hasta tener su valor mínimo en un modelo con una intensidad del campo magnético de 200 Gauss, para luego ir aumentando lentamente, casi en forma asintótica para modelos con campos de mayor intensidad.

En el caso en que la fuerza de Lorentz apunta hacia el interior de la estrella, vemos que el ancho equivalente de la línea aumenta, pero proporcionalmente menos que en el caso del modelo con una abundancia solar. Además, observamos que la máxima variación no ocurre cuando la intensidad del campo magnético es aproximadamente 200 Gauss (ver sección \$5.3. 
Tabla 7.3: Anchos equivalentes de las líneas de He I para modelos con una fracción de masa de He de 0.20 , con distintas intensidades del campo magnético y con las dos direcciones posibles de la fuerza de Lorentz.

\begin{tabular}{||l||l|l|l|l||l|l|l|l||}
\hline \multicolumn{4}{|c|}{} & F. de Lorentz hacia afuera & \multicolumn{3}{|l|}{ F. de Lorentz hacia adentro } \\
\hline $\mathrm{B}$ & 4026 & 4471 & 5015 & 5875 & 4026 & 4471 & 5015 & 5875 \\
\hline \hline $0 \mathrm{G}$ & 0.4207 & 0.1568 & 0.3924 & 0.4291 & 0.4207 & 0.1568 & 0.3924 & 0.4291 \\
\hline $10 \mathrm{G}$ & 0.4192 & 0.1563 & 0.3889 & 0.4275 & 0.4215 & 0.1572 & 0.3949 & 0.4300 \\
\hline $50 \mathrm{G}$ & 0.4131 & 0.1538 & 0.3737 & 0.4185 & 0.4232 & 0.1579 & 0.4021 & 0.4337 \\
\hline $100 \mathrm{G}$ & 0.4054 & 0.1504 & 0.3581 & 0.4103 & 0.4243 & 0.1583 & 0.4080 & 0.4372 \\
\hline $200 \mathrm{G}$ & 0.3946 & 0.1458 & 0.3414 & 0.4025 & 0.4255 & 0.1594 & 0.4150 & 0.4411 \\
\hline $300 \mathrm{G}$ & 0.3912 & 0.1442 & 0.3381 & 0.4022 & 0.4263 & 0.1596 & 0.4184 & 0.4428 \\
\hline $400 \mathrm{G}$ & 0.3919 & 0.1445 & 0.3402 & 0.4038 & 0.4267 & 0.1601 & 0.4200 & 0.4437 \\
\hline $500 \mathrm{G}$ & 0.3940 & 0.1455 & 0.3435 & 0.4056 & 0.4271 & 0.1599 & 0.4206 & 0.4439 \\
\hline $750 \mathrm{G}$ & 0.3989 & 0.1473 & 0.3505 & 0.4093 & 0.4271 & 0.1602 & 0.4201 & 0.4432 \\
\hline $1000 \mathrm{G}$ & 0.4020 & 0.1485 & 0.3548 & 0.4112 & 0.4271 & 0.1600 & 0.4185 & 0.4419 \\
\hline
\end{tabular}

sino que ocurre para intensidades cercanas a 750 Gauss.
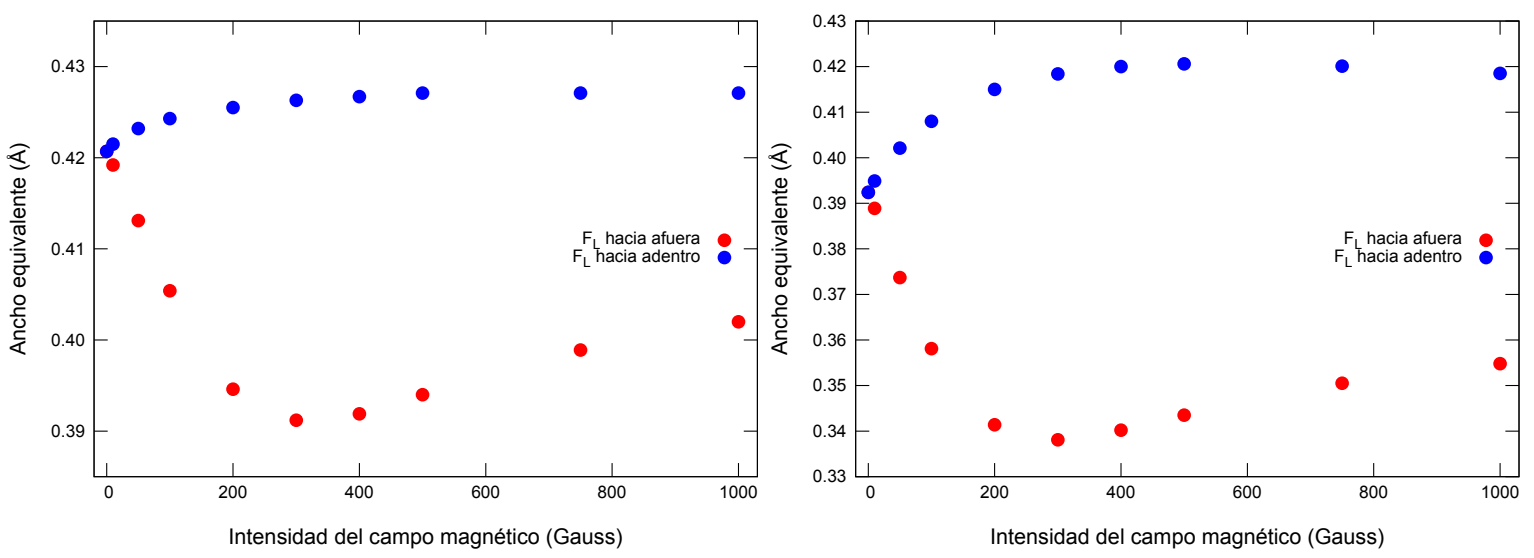

Figura 7.16: Anchos equivalentes de la líneas He I $\lambda 4526$ (izquierda) y He I $\lambda 5015$ (derecha) para distintas intensidades del campo magnético. Los puntos en rojo representan cuando la $F_{L}$ está dirigida hacia afuera y los azules cuando la $F_{L}$ está dirigida hacia adentro.

Este comportamiento puede observarse en la figura 7.16 para las líneas de las líneas He I $\lambda 4026$ y He I $\lambda 5015$. Encontramos que la variación del ancho equivalente de la línea de He I $\lambda 4026$ respecto a un modelo sin campo puede llegar a ser de un $4.4 \%$ cuando la fuerza de Lorentz se dirige hacia el exterior, y de un $1.5 \%$ si la fuerza de Lorentz apunta hacia el interior, para una estrella con un campo magnético de 1000 Gauss, Estos valores pueden ascender a $6.2 \%$ y $1.1 \%$, respectivamente, si el campo es de 200 Gauss,

Las variaciones son mayores aún para He I $\lambda 4471$ (de $5.3 \%$ y $2.0 \%$ cuando el campo es de 1000 Gauss y de $7.0 \%$ y $1.7 \%$ cuando es de 200 Gauss) y para He I $\lambda 5015$ (de $9.5 \%$ a $6.5 \%$ para 1000 Gauss de $13 \%$ a $5.7 \%$ para 200 Gauss). 
En el caso de aumentar la abundancia de He, no encontramos que las variaciones en los anchos equivalentes sean mayores. En cambio, al disminuir la abundancia de He, vemos que las variaciones se acentúan, como así también cuando calculamos modelos correspondientes a tipos espectrales más tardíos. Hemos realizado los cáculos para modelos de estrellas B5V pero con abundancia menor a la solar y verificamos que las variaciones son mayores.

Si bien mostramos que una disminusión del contenido de He superficial genera variaciones más apreciables en los perfiles de línea, estos cálculos consideran aún valores constantes de $n_{H e} / n_{H}$ y no se indican las causas que podrían contribuir a producir una distribución inhomogenea del contenido de He en la superficie de la estrella.

Por lo expuesto aquí, se hace necesario contar con otro mecanismo que posibilite la formación de zonas con abundancias de He bastante distintas en diferentes regiones de la estrella. Para ello, en los próximos capítulos, analizaremos los procesos de la difusión gravitatoria y cómo la fuerza de Lorentz podría contribuir a describir este fenómeno. 


\section{Capítulo 8}

\section{Difusión}

En la mayoría de los casos, los modelos de atmósferas de estrellas de secuencia principal se basan en la hipótesis que el contenido de los elementos químicos presentes en el medio está uniformemente distribuido en cada capa y es similar al de la materia primordial de la nube molecular donde nacieron las estrellas. Sin embargo, durante las etapas de la evolución estelar, las reacciones nucleares modifican la composición del interior estelar.

Para que la difusión tenga lugar en las atmósferas de las estrellas es necesario que las mismas sean prácticamente estables. Esto se debe a que una marcada convección no permite que la difusión tenga lugar en la estrella. Por otra parte, la rotación estelar, cuando ésta es rápida, hace que ocurran procesos de mezcla turbulenta enriqueciendo de esta manera a la atmósfera con isótopos del ciclo CNO Lyubimkov, 1984, 1988, 1989, 1991. Pero a pesar de las inestabilidades convectivas, habría posibilidad de que el mecanismo de difusión sea efectivo siempre y cuando las corrientes por convección y corrientes por circulación meridional sean menores a $10^{3} \mathrm{~cm} / \mathrm{s}$. En lo que respecta a las escalas de tiempo, la correspondiente a la difusión es menor que la escala de los tiempos evolutivos. Por ejemplo, bajo la presencia de un campo gravitatorio, la escala de tiempo para la difusión radiativa se estima en unos $10^{4}$ años Michaud, 1970.

Las condiciones para que el mecanismo de difusión ocurra se dan en las atmósferas de las estrellas CP, dado que son rotadores lentos y carecen de capas convectivas en la superficie. Además, en las estrellas CP, la existencia de los campos magnéticos puede también favorecer la estabilidad, dado que se conoce que éstos inhiben la convención en las regiones de las manchas solares; pero también pueden frenar el mecanismo de difusión de los iones en aquellas regiones donde $\omega_{c} \tau_{c}=1$.

Los procesos de sedimentación, es decir, difusión bajo la acción de un campo gravitatorio, hacen que los elementos pesados fluyan hacia abajo, mientras que los más livianos asciendan 
hacia la superficie. De esta manera, la estratificación gravitatoria es un mecanismo efectivo para separar distintos elementos químicos tanto en los interiores como en las atmósferas de las estrellas. Varios resultados teóricos han demostrado la importancia de la difusión en las estrellas peculiares Alecian y Stift 2006 Michaud 1970 y en la evolución estelar, como por ejemplo en el interior de las enanas blancas Althaus y Benvenuto, 2000, Althaus et al. 2009a banei et al. 2007, así como también en la descripción de las abundancias de los distintos elementos químicos en las estrellas WR Maeder, 1987.

Michaud 1970 discutió los efectos de la presión de radiación como una fuerza selectiva para favorecer la presencia de elementos pesados en la superficie de las estrella CP, ya que para que un elemento pesado no se hunda se debe ejercer sobre éste una fuerza con una intensidad comparable a la de la gravedad y en dirección opuesta. Michaud demostró que la fuerza de radiación permite esta selección de los elementos químicos pero la presencia de esta fuerza por sí sola no describe la formación de manchas o regiones confinadas donde un elemento químico particular presente una sobreabundancia o deficiencia.

Deberíamos entonces discutir si el efecto combinado de la difusión en presencia de un campo magnético permite determinar regiones con heterogeneidades en la atmósfera de una estrella dado que estas regiones estarán caracterizadas por el comportamiento de la gravedad efectiva (no uniforme) con la profundidad, por la latitud y por la dirección con la que actúa la fuerza de Lorentz. Para analizar este efecto, despreciaremos en una primera instancia los efectos de la fuerza de radiación, a fin de verificar qué variación porcentual aporta el campo magnético a los efectos de un campo eléctrico en presencia de un campo gravitatorio.

En nuestro caso modelaremos la difusión de una atmósfera constituida sólo por H y He para una estrella de tipo espectral B2V a fin de explicar las anomalías del He en la superficie donde los efectos de inercia han sido despreciados. Consideraremos además, que todas las especies de partículas en un mismo elemento de volumen tienen la misma temperatura $T$, donde $T$ puede ser una función del tiempo y de la posición. Además, suponemos que no hay una densidad de carga neta ni un flujo de masa resultante respecto del centro de masa del elemento de volumen. A partir de la ecuación de Boltzmann con colisiones de las partículas y tomando momentos respecto de la velocidad de la partícula, se obtienen entre otras, la ecuación de difusión y la ecuación del flujo de calor. A partir de esto, se puede determinar que el gradiente de presión depende de la suma de todas las fuerzas externas, en donde estas fuerzas son las debidas a la gravedad, a el campo eléctrico y a el campo magnético. Así, de acuerdo con Burgers 1969, tendremos la ecuación de difusión de la siguiente forma, 


$$
\begin{aligned}
\boldsymbol{\nabla} p_{s} & -\frac{\rho_{s}}{\rho} \boldsymbol{\nabla} p-n_{s} Z_{s} e \boldsymbol{E}-\left(\boldsymbol{j}_{\boldsymbol{s}}-\frac{\rho_{s}}{\rho} \boldsymbol{j}\right) \times \frac{\boldsymbol{B}}{c} \\
& =\sum_{t \neq s, t=1}^{N} K_{s t}\left(\boldsymbol{w}_{\boldsymbol{t}}-\boldsymbol{w}_{\boldsymbol{s}}\right)+\sum_{t \neq s, t=1}^{N} K_{s t} z_{s t} \frac{m_{t} \boldsymbol{r}_{\boldsymbol{s}}-m_{s} \boldsymbol{r}_{\boldsymbol{t}}}{m_{s t}}
\end{aligned}
$$

donde $p$ y $\rho$ son la presión total del gas y la densidad de masa, respectivamente, $e$ es la carga del electrón en uma y $c$ es la velocidad de la luz. $p_{s}, \rho_{s}, n_{s}, Z_{s}, m_{s}$, son la presión parcial, densidad de masa, densidad de número de partículas, carga media y masa, respectivamente, para la especie $s$. Las sumas se hacen sobre el índice $t$ con $t \neq s$. Definimos la masa de una mezcla binaria como $m_{s t}=m_{s}+m_{t}$. Los vectores $\boldsymbol{w}_{\boldsymbol{s}}, \boldsymbol{r}_{\boldsymbol{s}}$, y $\boldsymbol{j}_{\boldsymbol{s}}$, son la velocidad respecto del centro de masa del elemento de fluido, el flujo residual de calor, y la corriente de conducción considerando una velocidad del flujo de masa promedio, para la especie $s$, respectivamente. El vector $j_{s}$ está definido por:

$$
\boldsymbol{j}_{s}=n_{s} Z_{s} e \boldsymbol{w}_{\boldsymbol{s}}
$$

Las cantidades $\boldsymbol{E}$ y $\boldsymbol{B}$ son el campo eléctrico y el campo magnético, respectivamente, $\boldsymbol{j}$ es la corriente inducida total $\left(\sum_{s=1} \boldsymbol{j}_{\boldsymbol{s}}\right), K_{s t} \mathrm{y} z_{s t}$ son coeficientes resistivos, cuyas expresiones fueron dadas por Paquette et al. 1986), y que veremos más adelante.

La ecuación del flujo de calor está dada por la siguiente expresión:

$$
\begin{aligned}
\frac{5}{2} n_{s} k \nabla T & -\frac{n_{s} Z_{s} e}{c}\left(\boldsymbol{r}_{\boldsymbol{s}} \times \boldsymbol{B}\right) \\
& =-\frac{5}{2} \sum_{t \neq s, t=1}^{N} K_{s t} z_{s t} \frac{m_{t}}{m_{s t}}\left(\boldsymbol{w}_{\boldsymbol{t}}-\boldsymbol{w}_{\boldsymbol{s}}\right)-\frac{2}{5} K_{s s} z_{s s}^{\prime \prime} \boldsymbol{r}_{\boldsymbol{s}} \\
& -\sum_{t \neq s, t=1}^{N} \frac{K_{s t}}{m_{s t}^{2}}\left[3 m_{s}^{2}+m_{t}^{2} z_{s t}^{\prime}+\frac{4}{5} m_{s} m_{t} z_{s t}^{\prime \prime}\right] \boldsymbol{r}_{\boldsymbol{s}} \\
& +\sum_{t \neq s, t=1}^{N} K_{s t} \frac{m_{s} m_{t}}{m_{s t}^{2}}\left[3+z_{s t}^{\prime}-\frac{4}{5} z_{s t}^{\prime \prime}\right] \boldsymbol{r}_{\boldsymbol{t}}
\end{aligned}
$$

Las sumas son sobre el índice $t$ con $t \neq s$. Aquí, $k$ es la constante de Boltzmann, y $z_{s t}^{\prime}$ y $z_{s t}^{\prime \prime}$ son también coeficientes resistivos dados por Paquette et al. 1986.

Para una situación de equilibrio tendremos que no hay un flujo de masa neto respecto del centro de masa del elemento de volumen, así que la ecuación de la conservación de la masa se expresa de la siguiente forma: 


$$
\sum_{s=1}^{N} \rho_{s} \boldsymbol{w}_{s}=0
$$

y como no hay una corriente eléctrica neta, tendremos una ecuación para la conservación de la carga eléctrica:

$$
\sum_{s=1}^{N} Z_{s} n_{s} \boldsymbol{w}_{s}=0
$$

Todas las sumas son hasta $N$, donde $N$ es el número de todas las especies involucradas: iones, átomos neutros, y electrones. La ecuación 8.1 nos da un conjunto de $N-1$ ecuaciones lineales independientes Burgers 1969. A partir de la ecuación 8.2 obtenemos $N$ ecuaciones lineales, más dos ecuaciones adicionales definidas por las ecuaciones (8.3) y 8.4). Como hemos presentado en el capítulo \$3 la ecuación de equilibrio hidrostático en presencia de campos magnéticos puede escribirse como,

$$
\nabla P=\rho \boldsymbol{g}+\frac{1}{c} \boldsymbol{j} \times \boldsymbol{B}
$$

lo que es equivalente a definir

$$
\nabla p=\rho \boldsymbol{g}_{\text {eff }},
$$

donde $\boldsymbol{g}_{\boldsymbol{e f f}}$ es una gravedad efectiva que tiene en cuenta los efectos de la fuerza de Lorentz. Si además, se considerara la interacción entre fotones y partículas, las partículas adquieren una aceleración radiativa adicional $\boldsymbol{a}_{\boldsymbol{R}}$,

$$
\boldsymbol{a}_{\boldsymbol{R}}=\frac{\nabla p_{R}}{\rho}
$$

Siendo $\nabla p_{R}$ el gradiente de presión radiativa y $\rho$ la densidad de masa total. Esta aceleración es opuesta a la gravedad. A partir de las ecuaciones (8.5) y 8.6), la ecuación (8.1) puede escribirse de la siguiente forma:

$$
\begin{aligned}
\nabla p_{s} & -\rho_{s}\left(\boldsymbol{g}_{\boldsymbol{e f f}}-\boldsymbol{a}_{\boldsymbol{R}}\right)-n_{s} Z_{s} e \boldsymbol{E}-\boldsymbol{j}_{\boldsymbol{s}} \times \frac{\boldsymbol{B}}{c} \\
& =\sum_{t \neq s, t=1}^{N} K_{s t}\left(\boldsymbol{w}_{\boldsymbol{t}}-\boldsymbol{w}_{\boldsymbol{s}}\right)+\sum_{t \neq s, t=1}^{N} K_{s t} z_{s t} \frac{m_{t} \boldsymbol{r}_{\boldsymbol{s}}-m_{s} \boldsymbol{r}_{\boldsymbol{t}}}{m_{s t}}
\end{aligned}
$$

Dado que consideramos un modelo unidimensional (modelo plano paralelo), las partículas 
sólo pueden ascender o descender, como primera aproximación, por lo tanto el producto vectorial $\boldsymbol{j}_{\boldsymbol{s}} \times \boldsymbol{B}$, a excepción del polo magnético, está contenido en el plano normal a la coordenada radial y no tendrá componente en la dirección del versor $\check{\boldsymbol{r}}$. Luego, en un modelo unidimensional, la ecuación (8.7) toma la siguiente forma:

$$
\begin{aligned}
\frac{d p_{s}}{d r} & -\rho_{s}\left(g_{\text {eff }}-a_{R}\right)-n_{s} Z_{s} e E \\
& =\sum_{t \neq s, t=1}^{N} K_{s t}\left(w_{t}-w_{s}\right)+\sum_{t \neq s, t=1}^{N} K_{s t} z_{s t} \frac{m_{t} r_{s}-m_{s} r_{t}}{m_{s t}}
\end{aligned}
$$

En la ecuación (8.8), hemos considerado $\boldsymbol{w}_{\boldsymbol{s}}=w_{s} \check{\boldsymbol{r}}$ y $\boldsymbol{r}_{\boldsymbol{s}}=r_{s} \check{\boldsymbol{r}}$. De la misma forma, a partir de la ecuación 8.2 (ecuación del flujo de calor), podemos escribir:

$$
\begin{aligned}
\frac{5}{2} n_{s} k \frac{d T}{d r} & =-\frac{5}{2} \sum_{t \neq s, t=1}^{N} K_{s t} z_{s t} \frac{m_{t}}{m_{s t}}\left(w_{t}-w_{s}\right)-\frac{2}{5} K_{s s} z_{s s}^{\prime \prime} r_{s} \\
& -\sum_{t \neq s, t=1}^{N} \frac{K_{s t}}{m_{s t}^{2}}\left(3 m_{s}^{2}+m_{t}^{2} z_{s t}^{\prime}+\frac{4}{5} m_{s} m_{t} z_{s t}^{\prime \prime}\right) r_{s} \\
& +\sum_{t \neq s, t=1}^{N} K_{s t} \frac{m_{s} m_{t}}{m_{s t}^{2}}\left(3+z_{s t}^{\prime}-\frac{4}{5} z_{s t}^{\prime \prime}\right) r_{t}
\end{aligned}
$$

Como mencionamos anteriormente, el vector $\boldsymbol{r}_{\boldsymbol{s}} \times \boldsymbol{B}$ también está contenido en el plano normal a la coordenada radial. Del mismo modo, las ecuaciones (8.3) y (8.4) pueden escribirse como:

$$
\begin{aligned}
& \sum_{s=1}^{N} \mu_{s} n_{s} w_{s}=0 \\
& \sum_{s=1}^{N} Z_{s} n_{s} w_{s}=0
\end{aligned}
$$

En la ecuación 8.10, usamos que $\rho_{s}=n_{s} \mu_{s} M_{u}$, donde $\mu_{s}$ es el peso molecular para la especie $s$ y $M_{u}$ es la unidad de masa atómica.

El nuevo sistema a resolver incluye a las ecuaciones 8.8, 8.9, 8.10 y 8.11, el cual está constituido por $(2 N+1)$ ecuaciones con $(2 N+1)$ incógnitas. $N$ incógnitas corresponden a las variables $w_{s}, N$ a las $r_{s}$ y la restante al campo eléctrico $E$. 


\subsection{Método de solución}

Para resolver el sistema de ecuaciones 8.8, 8.9, 8.10 y 8.11, utilizamos el método propuesto por Iben y MacDonald 1985, donde descompone a la velocidad relativa, $w_{s}$, de la siguiente manera:

$$
w_{s}=w_{s}^{g}-\sum_{t=1}^{N_{\text {ion }}} \sigma_{s t} \frac{d \ln n_{t}}{d r},
$$

donde $w_{s}^{g}$ es la velocidad correspondiente a la componente gravitatoria. Los $\sigma_{s t}$ son los coeficientes del desarrollo de $w_{s}$, que al igual que $w_{s}^{g}$, debemos determinar, y son necesarios para calcular las nuevas densidades de partículas de las especies químicas difundidas. Estos coeficientes son las componentes de la velocidad de la especie $s$ debida a la interacción con las partículas de la especie $t$. La suma es sobre las componentes debidas a los gradientes de las densidades de partículas de todas las especies químicas que se difunden. La suma es sólo sobre los iones, con $N_{i o n}=N-1$. Análogamente, podemos escribir una expresión similar para el flujo de calor residual $r_{s}$ y el campo eléctrico $E$, por ejemplo,

$$
\begin{gathered}
r_{s}=r_{s}^{g}-\sum_{t=1}^{N_{\text {ion }}} \eta_{s t} \frac{d \ln n_{t}}{d r} \\
E=E^{g}-\sum_{t=1}^{N_{\text {ion }, 0}} \zeta_{t} \frac{d}{d r}\left(\ln n_{t}\right)
\end{gathered}
$$

Aquí, $r_{s}^{g}$ es el flujo de calor residual correspondiente a la componente gravitatoria, y los $\eta_{s t}$ cumplen, para el flujo de calor residual, el mismo papel que los coeficientes $\sigma_{s t}$ para la velocidad. De la misma forma, $E^{g}$ es la componente del campo eléctrico asociada a la gravedad y los $\zeta_{t}$ son análogos a $\eta_{s t}$ y $\sigma_{s t}$. Notemos que las ecuaciones 8.12, 8.13) y 8.14 dependen de $n_{t}$, y estas cantidades corresponden al paso previo en el cual se calculan. Es decir, las cantidades $w_{s}^{g}, \sigma_{s t}, r_{s}^{g}, \eta_{s t}, E^{g}$ y $\zeta_{t}$ son calculadas al comienzo del paso temporal en el tiempo $t_{0}$. Una vez que hemos obtenido los flujos de velocidades $w_{s}$, los flujos de calor residual $r_{s}$, y el campo eléctrico $E$, debemos resolver la ecuación de conservación de masa:

$$
\frac{\partial \rho_{s}}{\partial t}+\nabla \cdot \Phi_{s}=0
$$

donde $\Phi_{s}$ es el vector flujo de masa definido por:

$$
\boldsymbol{\Phi}_{\boldsymbol{s}}=m_{s} n_{s} \boldsymbol{w}_{\boldsymbol{s}}=n_{s} \mu_{s} M_{u} \boldsymbol{w}_{\boldsymbol{s}}
$$

donde, 


$$
\rho_{s}=m_{s} n_{s}=\mu_{s} M_{u} n_{s}
$$

es la densidad expresada en términos del número de partículas por $\mathrm{cm}^{3}$. En simetría esférica, y usando las ecuaciones 8.16 y 8.17, la ecuación 8.15 pasa a ser:

$$
\frac{\partial n_{s}}{\partial t}+\frac{1}{r^{2}} \frac{\partial}{\partial r}\left(r^{2} n_{s} w_{s}\right)=0
$$

La ecuación (8.18) sólo tiene en cuenta la difusión de los iones ya que la masa de los electrones es 1840 veces menor que la de los iones de H. Los $w_{s}^{g}, \sigma_{s t}, r_{s}^{g}, \eta_{s t}, E^{g}$ y $\zeta_{t}$ son encontrados a partir de las ecuaciones (8.8) a 8.11) mediante una matriz solución sencilla. Para la ecuación (8.18) sólo nos interesan los $w_{s}$.

\subsection{Condiciones de borde}

Si bien la ecuación 8.18 es una ecuación diferencial de primer orden, al momento de resolverla numéricamente, como veremos en la sección 98.3 la misma será transformada en una ecuación diferencial de segundo orden en $r$, y por ende, deberemos imponer dos condiciones de contorno. En la parte exterior de la atmósfera, el número de partículas tiende a cero,

$$
n_{s}=0
$$

Sin embargo, la condición impuesta es $\partial x_{s} / \partial r=0$, es decir, se llega a la superficie con densidad nula pero con abundancia fraccional de masa constante. En la base de la atmósfera, la condición es:

$$
\frac{d n_{s}}{d r}=0
$$

con lo que tendremos que $n_{s}$ es constante, donde $s=1, \ldots, N_{\text {ion }}$.

\subsection{Método numérico}

Para resolver la ecuación 8.18, emplearemos el método numérico de eliminación de variables hacia adelante y sustitución hacia atrás (forward and backward substitution). Este método es similar al utilizado por Iben y MacDonald 1985 y para ello es necesario escribir la ecuación 8.18 como una ecuación diferencial de segundo orden.

A partir de la ecuación 8.12, la ecuación 8.18 puede reescribirse como: 


$$
\begin{aligned}
\frac{\partial n_{s}}{\partial t} & =-\frac{1}{r^{2}} \frac{\partial}{\partial r}\left(r^{2} n_{s} w_{s}\right) \\
& =-\frac{1}{r^{2}} \frac{\partial}{\partial r}\left[r^{2}\left(w_{s}^{g} n_{s}-\sum_{t}^{N_{\text {ion }}} \frac{n_{s}}{n_{t}} \sigma_{s t} \frac{\partial n_{t}}{\partial r}\right)\right]
\end{aligned}
$$

Esta ecuación es resuelta mediante un método conservativo semi-implícito de diferencias finitas, de primer orden en el tiempo y de segundo orden en la variable espacial $r$. Como hemos mencionado, los $w_{s}$ son calculados previamente al comienzo del paso temporal $t_{0}$ con las densidades $n_{s}$ conocidas. El paso temporal es $\Delta y=t-t_{0}$. Escribiendo

$$
d\left(\frac{r^{3}}{r}\right)=r^{2} d r
$$

el operador derivada se convierte en,

$$
\frac{1}{r^{2}} \frac{\partial}{\partial r}=\frac{\partial}{\partial\left(r^{3} / 3\right)}
$$

A las derivadas en $r$ las centramos en la capa $k$, con una capa por encima identificada con el índice $k+1 / 2$, y otra por debajo con un índice $k-1 / 2$. La derivada discretizada será:

$$
\frac{\partial}{\partial\left(r^{3} / 3\right)} \quad \rightarrow \quad \frac{3}{r_{k+1 / 2}^{3}-r_{k-1 / 2}^{3}}=\frac{1}{\Delta V_{k}}
$$

es decir,

$$
\Delta V_{k}=\frac{r_{k+1 / 2}^{3}-r_{k-1 / 2}^{3}}{3}
$$

con,

$$
r_{k+1 / 2}^{3}=\frac{r_{k}^{3}+r_{k+1}^{3}}{2}
$$

La grilla es tal que el índice $k=0$ corresponde a la base de la atmósfera, y el índice $k=K$ corresponde la borde externo. Con estas consideraciones y trabajando algebraicamente, las ecuaciones en diferencias finitas toman la siguiente forma:

$$
\begin{aligned}
n_{s, k}-n_{s, k}^{0} & =\frac{\Delta t}{\Delta V_{k}}\left\{-\left(r_{k+1 / 2}^{2} w_{s, k+1 / 2}^{g, 0} n_{k+1 / 2}-r_{k-1 / 2}^{2} w_{s, k-1 / 2}^{g, 0} n_{k-1 / 2}\right)\right. \\
& +\sum_{t}\left[r_{k+1 / 2}^{2} S_{s t, k+1 / 2}^{0} \frac{\left(n_{t, k+1}-n_{t, k}\right)}{\Delta r_{k+1 / 2}}\right. \\
& \left.\left.-r_{k-1 / 2}^{2} S_{s t, k-1 / 2}^{0} \frac{\left(n_{t, k}-n_{t, k-1}\right)}{\Delta r_{k-1 / 2}}\right]\right\},
\end{aligned}
$$


donde los supraíndices 0 indican que la cantidad está evaluada al comienzo del intervalo de tiempo $\Delta t$. Las cantidades sin el supraíndice 0 son desconocidas y su valor es calculado al final del intervalo de tiempo. Los radios en el centro de la región $k, r_{k}$, no cambian durante el intervalo de tiempo. Las otras cantidades de la ecuación 8.23 están definidas por:

$$
\begin{gathered}
n_{s, k+1 / 2}=\frac{n_{s, k}+n_{s, k+1}}{2} \\
S_{s t, k+1 / 2}^{0}=\frac{n_{s, k+1 / 2}^{0} \sigma_{s t, k+1 / 2}^{0}}{n_{t, k+1 / 2}^{0}}
\end{gathered}
$$

Las cantidades $w_{s, k+1 / 2}^{g, 0}$ y $\sigma_{s t, k+1 / 2}^{0}$ son evaluadas en el tiempo $t=t_{0}$.

El sistema ecuaciones 8.23 es lineal en las incógnitas $n$ y, para cada región $k$, pueden reescribirse en forma matricial. Entonces nos queda el siguiente sistema de ecuaciones:

$$
-\mathrm{A}_{\mathrm{k}} \boldsymbol{n}_{k+1}+\mathrm{B}_{\mathrm{k}} \boldsymbol{n}_{k}-\mathrm{C}_{\mathrm{k}} \boldsymbol{n}_{k-1}=D_{k}
$$

donde A, B y $\mathbf{C}$ son matrices y $\boldsymbol{n}_{\boldsymbol{k}}$ y $\boldsymbol{D}_{\boldsymbol{k}}$ son vectores. Las componentes del vector $\boldsymbol{n}_{\boldsymbol{k}}$ son las densidades del número de partículas de las especies químicas a difundir, en la región o capa k-ésima. Las condiciones de contorno 8.19 y 8.20 completan el sistema, y dado que los cálculos requieren integraciones separadas en cada capa de la atmósfera, entonces, la condición 8.20 es reemplazada por el requerimiento de que la fracción de masa de cada especie sea uniforme a través de la atmósfera.

La condición de contorno en el borde exterior nos conduce a una ecuación diferencial que también se discretiza y nos lleva a un sistema matricial de la forma,

$$
-\mathrm{A}_{0} n_{1}+\mathrm{B}_{0} n_{0}=D_{0}
$$

donde $\boldsymbol{n}_{\mathbf{0}}$ es el vector de la densidad del número de partículas en la capa interior del modelo de atmósfera. Las ecuaciones 8.26 pueden resolverse mediante una generalización simple de la técnica estándar para la solución de los problemas de flujo de calor mediante el procedimiento de eliminación de Gauss Richtmyer y Morton, 1994.

Trabajando algebraicamente, la ecuación 8.27 puede reescribirse como,

$$
n_{0}=\mathrm{B}_{0}^{-1} \mathrm{~A}_{0} n_{1}+\mathrm{B}_{0}^{-1} D_{0}
$$

y definiendo,

$$
\begin{aligned}
\mathbf{E}_{\mathbf{0}} & =\mathbf{B}_{\mathbf{0}}^{-1} \mathbf{A}_{\mathbf{0}} \\
\boldsymbol{F}_{\mathbf{0}} & =\mathrm{B}_{\mathbf{0}}^{-1} \boldsymbol{D}_{\mathbf{0}}
\end{aligned}
$$


finalmente la ecuación 8.27 nos queda como,

$$
n_{0}=\mathrm{E}_{0} n_{1}+F_{0}
$$

Análogamente, definimos las matrices $\mathbf{E}_{\mathbf{k}}$ y los vectores $\boldsymbol{F}_{\boldsymbol{k}}$, de tal forma que:

$$
\boldsymbol{n}_{\boldsymbol{k}}=\mathbf{E}_{\mathbf{k}} \boldsymbol{n}_{\boldsymbol{k}+\mathbf{1}}+\boldsymbol{F}_{\boldsymbol{k}}, \quad K-1 \geq k \geq 1
$$

donde $K$ es el número de capas en que hemos dividido nuestro modelo de atmósfera. Las ecuaciones 8.26 nos dan relaciones recursivas para $\mathbf{E}_{\mathbf{k}}$ y $\boldsymbol{F}_{\boldsymbol{k}}$,

$$
\begin{aligned}
& \mathbf{E}_{\mathrm{k}}=\mathbf{G}_{\mathrm{k}}^{-1} \mathbf{A}_{\mathrm{k}}, \\
& \boldsymbol{F}_{\boldsymbol{k}}=\mathbf{G}_{\mathrm{k}}^{-1}\left(\boldsymbol{D}_{\boldsymbol{k}}+\mathbf{C}_{\mathrm{k}} \boldsymbol{F}_{\boldsymbol{k}-\mathbf{1}}\right)
\end{aligned}
$$

donde

$$
\mathrm{G}_{\mathrm{k}}=\mathrm{B}_{\mathrm{k}}-\mathrm{C}_{\mathrm{k}} \mathrm{E}_{\mathrm{k}-1}
$$

De la misma manera que antes, la condición de contorno en el borde interior de la atmósfera nos da las ecuaciones para la capa $k=K$ de la atmósfera.

$$
\mathrm{B}_{\mathrm{K}} n_{K}-\mathrm{C}_{\mathrm{K}} n_{K-1}=D_{K}
$$

Dado que $\mathbf{E}_{\mathbf{K}}=0$, tendremos que $\boldsymbol{n}_{\boldsymbol{K}}=\boldsymbol{F}_{\boldsymbol{K}}$. Los $\boldsymbol{n}_{\boldsymbol{k}}$ restantes se obtienen por recurrencia a partir de la ecuación 8.30. En general este método de resolución de las relaciones de recurrencia 8.26 es bastante estable, siendo por ende los determinantes de las matrices $\mathbf{G}$ y $(\mathbf{B}-\mathbf{A}-\mathbf{C})$ mayores que cero. Eventualmente en el caso en que alguna de las matrices se singularice (es decir que su determinante se haga nulo), esto se puede solucionar cambiando el paso del tiempo de difusión. El motivo de esta reducción del paso es debido a que, si el intervalo de tiempo es muy grande, la aproximación lineal deja de ser buena y como consecuencia se producen matrices singulares. Reduciendo el intervalo de tiempo, volvemos a estar en la región de validez de la aproximación lineal.

\subsection{Coeficientes difusivos y resistivos}

Hemos visto que para resolver las ecuaciones 8.8, 8.9, 8.10 y 8.11 debemos conocer un conjunto de coeficientes de difusión. Para describirlos, en este trabajo utilizaremos el 
formalismo de Burgers 1969. En este formalismo, los coeficientes de difusión se expresan en términos de las denominadas integrales de colisión y dependen del tipo de interacción entre las distintas partículas que colisionan. Las colisiones están dominadas por encuentros binarios.

Para describir la interacción de dos partículas cargadas embebidas en un plasma, el uso del potencial de Coulomb apantallado, permite en principio, una buena descripción de los efectos colectivos de las partículas vecinas y evita el tener que definir una distancia de corte ficticia (longitud de Debye) necesaria en el uso de un potencial coulombiano puro se evita de esta manera que la sección transversal de interacción diverja. La desventaja de este método es que no pueden ser calculadas analíticamente.

Aquí reproduciremos las fórmulas básicas para calcular los coeficientes de difusión $D_{s t}$ por colisión entre partículas de las especies $s$ y $t$ (ver Paquette et al. 1986). De acuerdo con Chapman y Cowling 1970, la primera aproximación para el coeficiente de difusión es,

$$
\left[D_{s t}\right]_{1}=\frac{3 E}{2 n m}
$$

donde $n=n_{s}+n_{t}$ es la densidad del número total de partículas, y $m=m_{s}+m_{t}$ es la suma de las masas de las partículas de las especies $s$ y $t$. Además,

$$
E=\frac{k_{B} T}{8 M_{s} M_{t} \Omega_{s t}^{(11)}}
$$

con $M_{i}=m_{i} / m(\operatorname{para} i=s, t)$.

Las cantidades, $\Omega_{s t}^{(i j)}$, son las llamadas integrales de colisión Paquette et al. 1986 y vienen dadas por:

$$
\Omega_{s t}^{(i j)}=\left(\frac{k_{B} T}{2 \pi m M_{s} M_{t}}\right)^{1 / 2} \int_{0}^{\infty} e^{-g^{2}} g^{2 j+3} \phi_{s t}^{(i)} d g,
$$

con

$$
\phi_{s t}^{(i)}=2 \pi \int_{0}^{\infty}\left(1-\cos ^{i} \chi_{s t}\right) b d b
$$

y

$$
\chi_{s t}=\pi-2 \int_{r_{s t}^{\min }}^{\infty} b d r\left[r^{2}\left(1-\frac{b^{2}}{r^{2}}-\frac{V_{s t}(r)}{g^{2} k_{B} T}\right)^{1 / 2}\right]^{-1}
$$

donde $V_{s t}(r)$ es el potencial de interacción y $r_{s t}^{\min }$ es la distancia de mínima de aproximación dada por la solución de la siguiente ecuación: el parámetro de impacto, es decir, es la distancia de la aproximación más cercana a la solución de la ecuación: 


$$
1-\frac{b^{2}}{\left(r_{s t}^{\min }\right)^{2}}-\frac{V_{s t}\left(r_{s t}^{\min }\right)}{g^{2} k_{B} T}=0
$$

Estas integrales describen las colisiones clásicas entre partículas de especies $s$ y $t$ que interactúan mediante un potencial $V_{s t}(r)$. Físicamente, los $\Omega_{s t}^{(i j)}$ están relacionados con las secciones eficaces totales después de integrar sobre una distribución Maxwelliana de velocidades, donde $g$ es una velocidad adimensional. Los $\phi_{s t}^{(i)}$ son las secciones eficaces colisionales para una dada energía. La integración se realiza sobre el parámetro de impacto $b$. La cantidad $\chi_{s t}$ es el ángulo de scattering que se calcula integrando sobre la distancia entre las partículas colisionantes.

En ese trabajo, las ecuaciones de difusión incluyen los denominados 4 coeficientes resistivos usados por Burgers 1969, los cuales están definidos por:

$$
K_{s t}=x_{s} x_{t} n \frac{k_{B} T}{\left[D_{s t}\right]_{1}},
$$

donde $\left[D_{s t}\right]_{1}$ está dado por la ecuación 8.36, y

$$
\begin{aligned}
& z_{s t}=-C, \\
& z_{s t}^{\prime}=-2 B+2.5, \\
& z_{s t}^{\prime \prime}=5 A,
\end{aligned}
$$

con

$$
\begin{gathered}
A=\frac{\Omega_{s t}^{(22)}}{5 \Omega_{s t}^{(11)}}, \\
B=\frac{\left(5 \Omega_{s t}^{(12)}-\Omega_{s t}^{(13)}\right)}{5 \Omega_{s t}^{(11)}}, \\
C=\left(\frac{2 \Omega_{s t}^{(12)}}{5 \Omega_{s t}^{(11)}}\right)-1,
\end{gathered}
$$

y donde los $\Omega_{s t}^{(i j)}$ están definidos por la ecuación 8.38. Los valores de estos coeficientes son necesarios para poder resolver el sistema de ecuaciones 8.8, 8.9, 8.10 y 8.11. 


\subsection{Evaluación de las integrales de colisión, ajustes analíticos}

Para aplicaciones de la difusión a plasmas estelares, las integrales de colisión para un potencial coulombiano estático apantallado pueden ser evaluadas numéricamente. Pero claramente, es más conveniente disponer de una representación analítica de los resultados, y en consecuencia, realizar una serie de ajustes para las integrales de colisión. A tal fin, se definen las integrales de colisión adimensionales

$$
F_{s t}^{i j}=\frac{\Omega_{s t}^{i j}}{\varepsilon_{s t}}
$$

donde

$$
\varepsilon_{s t}=\pi\left(\frac{Z_{s} Z_{t} e^{2}}{2 k_{B} T}\right)^{2}\left(\frac{k_{B} T}{2 \pi m M_{s} M_{t}}\right)^{1 / 2}
$$

Los valores de estas integrales dependen únicamente de $Z_{s}, Z_{t}, \lambda$, y $T$. $\lambda$ es el valor mayor entre la longitud de Debye definida por

$$
\lambda_{D}=\left(\frac{k_{B} T}{4 \pi e^{2} \sum_{i}^{N} n_{i} Z_{i}^{2}}\right)^{1 / 2}
$$

y la separación media entre los iones dada por Iben y MacDonald 1985 es,

$$
a_{0}=\left(\frac{3}{4 \pi \sum_{i}^{N_{i o n}} n_{i}}\right)^{1 / 3}
$$

Para hacer una correspondencia con el potencial coulombiano puro en el límite de densidad cero, se elige una combinación de estas variables tal que,

$$
\gamma_{s t}=\frac{4 k_{B} T \lambda}{Z_{s} Z_{t} e^{2}}
$$

En función de estos parámetros adimensionales, Muchmore 1980 sugiere emplear una variable independiente $\psi_{s t}$ que permite realizar el ajuste de las integrales de colisión adimensionales, dada por:

$$
\psi_{s t}=\ln \left[\ln \left(1+\gamma_{s t}^{2}\right)\right]
$$

La forma doble logarítmica permite cubrir un amplio rango de condiciones estelares, al contrario del rango relativamente pequeño que presenta $\psi_{s t}$. Formalmente, este ajuste es 
válido en el rango $-7.0 \leq \psi_{s t} \leq \infty$. Paquette et al. 1986) sugieren dividir este intervalo en 3 regiones.

La primera región corresponde a

$$
-7.0 \leq \psi_{s t} \leq 3.0
$$

Aquí la dependencia funcional es complicada y se deben usar funciones Spline cúbicas. Para ello, Paquette et al. 1986 consideraron 50 sub-intervalos equiespaciados tales que $\psi_{\text {st }}$ está en el intervalo n-ésimo cuando

$$
\psi_{s t}(n) \leq \psi_{s t} \leq \psi_{s t}(n+1)
$$

donde $\psi_{s t}(n)$ y $\psi_{s t}(n+1)$ son los valores de la variable independiente en los bordes del intervalo. Con estas definiciones, las integrales adimensionales de colisión estarán dadas por:

$$
\begin{aligned}
\ln F_{s t}^{(1 j)} & =c_{1 n}^{(j)}\left[\psi_{s t}(n+1)-\psi_{s t}\right]^{3}+c_{2 n}^{(j)}\left[\psi_{s t}-\psi_{s t}(n)\right]^{3} \\
& +c_{3 n}^{(j)}\left[\psi_{s t}(n+1)-\psi_{s t}\right]+c_{4 n}^{(j)}\left[\psi_{s t}-\psi_{s t}(n)\right] \quad(j=1,3)
\end{aligned}
$$

y

$$
\begin{aligned}
\ln F_{s t}^{(22)} & =d_{1 n}\left[\psi_{s t}(n+1)-\psi_{s t}\right]^{3}+d_{2 n}\left[\psi_{s t}-\psi_{s t}(n)\right]^{3} \\
& +d_{3 n}\left[\psi_{s t}(n+1)-\psi_{s t}\right]+d_{4 n}\left[\psi_{s t}-\psi_{s t}(n)\right]
\end{aligned}
$$

Los valores de los coeficientes $c_{l n}^{(j)}$ y $d_{l n}(l=1,4)$ están listados en las tablas B.1 a B.8 que se encuentran en el Anexo I de esta tesis. Se debe distinguir entre los casos de potencial repulsivo (colisiones ión-ión) y potencial atractivo (colisiones electrón-ión).

La segunda región corresponde a,

$$
3.0 \leq \psi_{s t} \leq 4.0
$$

para $\psi_{s t} \geq 2.7$, las cantidades $F_{s t}^{(i j)}$ se vuelven proporcionales a $\ln \left(1+\gamma_{s t}^{2}\right)$, por ejemplo a $e^{\psi_{s t}} \mathrm{y}$ en estos casos, se puede desarrollar una fórmula analítica exacta.

Para el potencial repulsivo se encuentran las siguientes expresiones,

$$
\begin{aligned}
& F_{s t}^{(11)}=1.00141 e^{\psi_{s t}}-3.18209, \\
& F_{s t}^{(12)}=0.99559 e^{\psi_{s t}}-1.29553, \\
& F_{s t}^{(13)}=1.99814 e^{\psi_{s t}}-0.64413, \\
& F_{s t}^{(22)}=1.99016 e^{\psi_{s t}}-4.56958,
\end{aligned}
$$


y para el caso del potencial atractivo tenemos,

$$
\begin{aligned}
& F_{s t}^{(11)}=1.01101 e^{\psi_{s t}}-3.19815 \\
& F_{s t}^{(12)}=1.04230 e^{\psi_{s t}}-1.89637 \\
& F_{s t}^{(13)}=2.15672 e^{\psi_{s t}}-2.81038 \\
& F_{s t}^{(22)}=2.08699 e^{\psi_{s t}}-5.81444
\end{aligned}
$$

Finalmente, la tercera región corresponde a,

$$
\psi_{s t} \geq 4.0
$$

En esta región, el plasma está tan débilmente acoplado, que los efectos de apantallamiento producen pequeñas diferencias entre las secciones eficaces de los casos con potenciales atractivos y repulsivos. Con una muy buena aproximación, las ecuaciones 8.56 pueden usarse en ambos casos (Paquette et al. 1986. 


\section{Capítulo 9}

\section{Resultados de los modelos con difusión}

En este capítulo mostraremos los resultados de aplicar los procesos de difusión atómica a modelos de atmósferas con campos magnéticos.

Los modelos que hemos calculado corresponden a distintas latitudes magnéticas, ya que, como hemos mostrados en capítulos anteriores, la gravedad efectiva es fuertemente dependiente del ángulo de latitud magnética. De esta manera tratamos de buscar regiones en donde la presencia de un campo magnético favorezca la creación de zonas con sobreabundancia o deficiencia de He.

\subsection{Efectos de la difusión sobre los elementos químicos}

Primeramente analizaremos los efectos de la difusión sobre la distribución de elementos químicos a lo largo de la atmósfera de un modelo en ausencia de campos magnéticos. Como mostramos anteriormente, los modelos calculados en los polos magnéticos, considerando la presencia de campos magnéticos, se comportan como si el campo fuera nulo.

Cabe destacar, que a medida que ocurre la difusión, se consideró el cambio de la ionización con la temperatura y la profundidad óptica. La relación de elementos ionizados se calculó usando la Ley de Saha.

En la figura 9.1 mostramos la fracción de masa de cuatro especies químicas, H II, He I, He II y He III, en función de la profundidad óptica de Rosseland. Se muestra la distribución de la fracción de masa para cada especie, a partir de un tiempo inicial cero hasta 100000 años con un paso de 10000 años. En nuestros modelos, el paso de tiempo con el cual vamos calculando la difusión es de 1000 años. Nosotros consideraremos que los procesos de 
difusión se estabilizaron (es decir, que el modelo convergió) cuando las variaciones de las especies químicas difundidas es menor al $1 \%$ entre dos modelos de tiempos consecutivos. Para los modelos de una estrella B2V esto ocurre aproximadamente a los 100000 años. Más allá de este tiempo, la difusión no tiene lugar, como consecuencia aparecen inestabilidades numéricas que producen valores erróneos en los números de partículas.
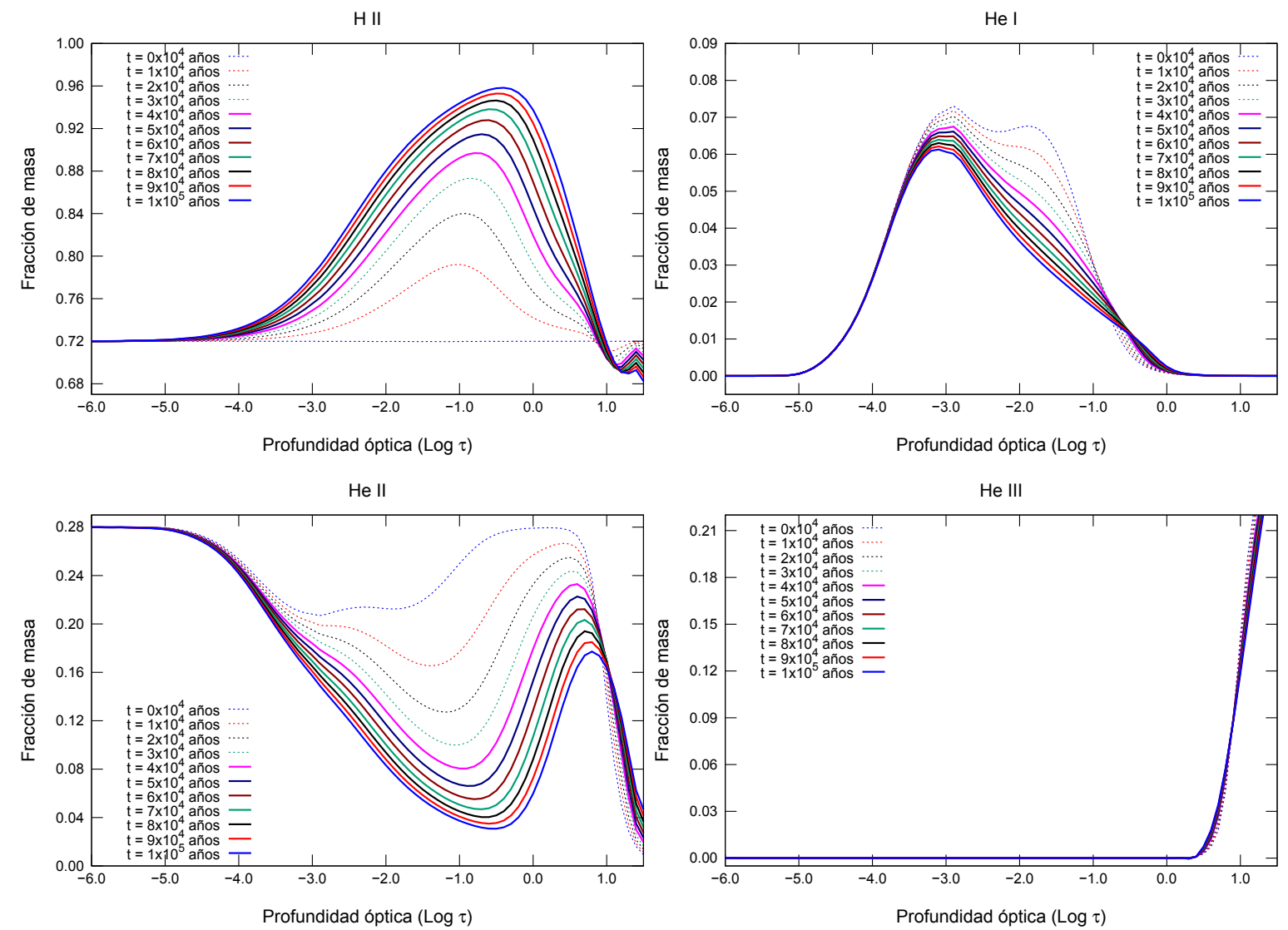

Figura 9.1: Fracción de masa de las distintas especies químicas en función de la profundidad óptica de Rosseland $(\log \tau)$, para distintos tiempos. Arriba izquierda: H II. Arriba derecha: He I. Abajo izquierda: He II. Abajo derecha: He III.

La línea punteada en color azul corresponde a la fracción de masa inicial. Lo primero que observamos es que en las capas interiores de la atmósfera no hay casi difusión de ninguna de las especies químicas consideradas. Prácticamente para $\log \tau>0.6$ no se observan cambios dado que no incluimos el interior. La abundancia de He III es casi nula, y sólo está presente en estas capas más internas de la atmósfera y por ende las cantidades difundidas de esta especie son pequeñas y casi no se detectan variaciones. Tampoco se observan variaciones en las regiones más externas de la atmósfera, a partir de valores de $\log \tau<-4$, aproximadamente. En el caso del H I, dado que para las temperaturas de nuestros modelos el H está casi todo ionizado, sólo hay pequeñas cantidades en las capas intermedias de la atmósfera y las variaciones producto de la difusión son pequeñas.

En el panel superior izquierdo, en donde mostramos la fracción de masa de H II, vemos que 
la abundancia de $\mathrm{H}$ ionizado va aumentando, principalmente en $\log \tau \approx-0.3$. Casi a la misma profundidad óptica, ocurre lo contrario con el He ionizado una vez (gráfico de abajo a la izquierda), que vé disminuida su abundancia fraccional en esa región. Algo similar ocurre con el He neutro, presente en menor proporción, aunque la mayor disminución ocurre para $\log \tau \approx-0.7$.

En la figura 9.2 graficamos las fracciones de masa iniciales y finales de las cuatro especies químicas todas juntas para poder compararlas. Con las líneas de trazo entrecortado mostramos las fracciones de masa a $t=0$ años, y con las líneas de trazo lleno, las correspondientes a $t=100000$ años. Comparadas con la misma escala, se nota que las variaciones mayores son en el $\mathrm{H}$ y He ionizado, aunque en forma relativa, el He neutro varía más que el H ionizado.

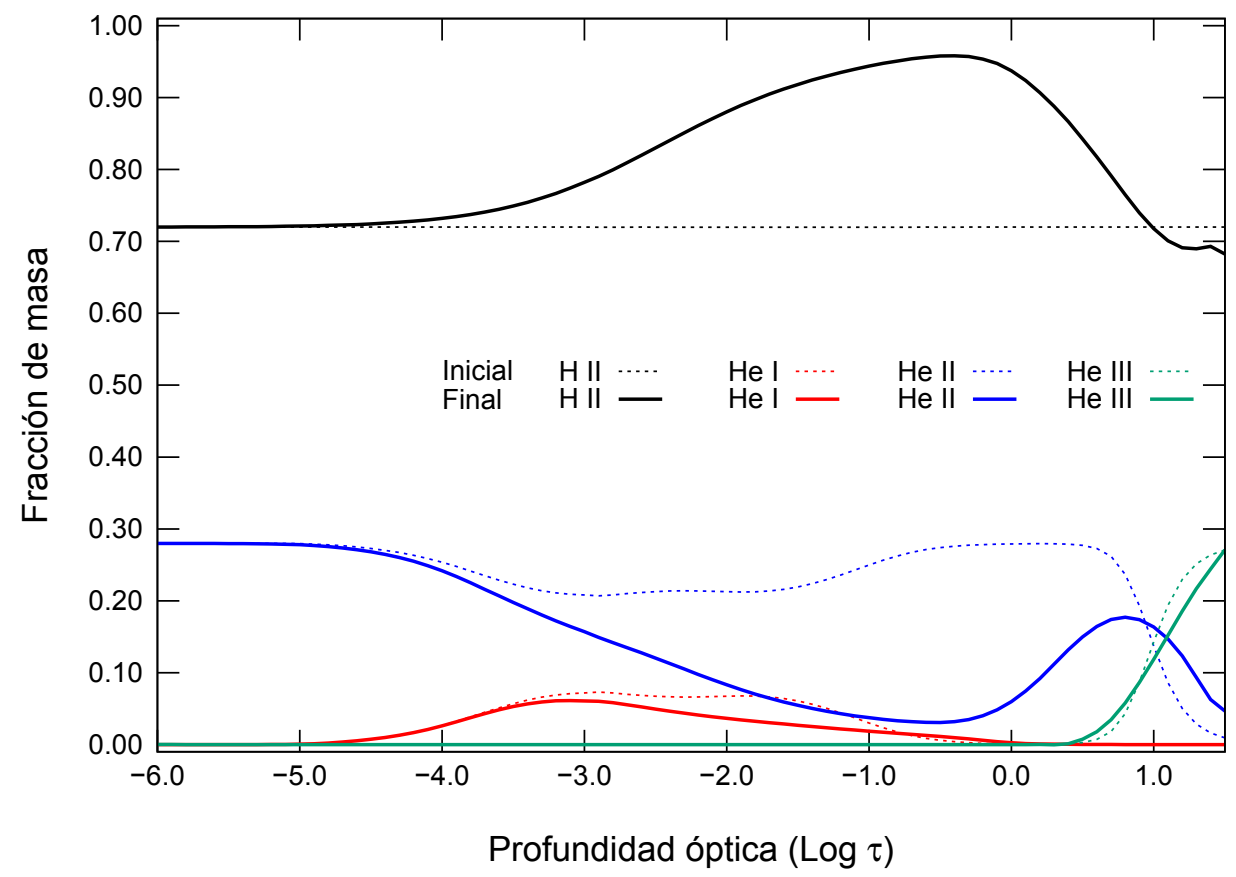

Figura 9.2: Fracción de masa de las distintas especies químicas en función de la profundidad óptica de Rosseland $(\log \tau)$. Las líneas de trazo lleno corresponden a las fracciones de masa iniciales y las entrecortadas a las fracciones de masa después de 100000 años. Las especies graficadas son H II (negro), He I (rojo), He II (azul) y He III (verde).

El código de difusión que hemos desarrollado, también nos da la densidad de partículas de cada especie. En estos casos, no hemos incluido los gráficos correspondientes, ya que muestran un comportamiento similar.

\subsection{Efectos de la latitud magnética sobre la difusión en presencia de campos magnéticos}

Como vimos, para una misma intensidad del campo magnético, la gravedad efectiva cambia con la latitud magnética. En esta sección veremos cómo la difusión de las distintas especies 
cambia al considerar modelos en distintas latitudes magnéticas. Además, la dirección de la fuerza de Lorentz también afecta la marcha de la gravedad efectiva y, por lo tanto, también afectará a los procesos difusivos.

En las figuras 9.3, 9.4 y 9.5 mostramos la fracción de masa en función de la profundidad óptica de H II, He I y He II, respectivamente, para el polo y ecuador magnético y para una latitud intermedia a $45^{\circ}$.
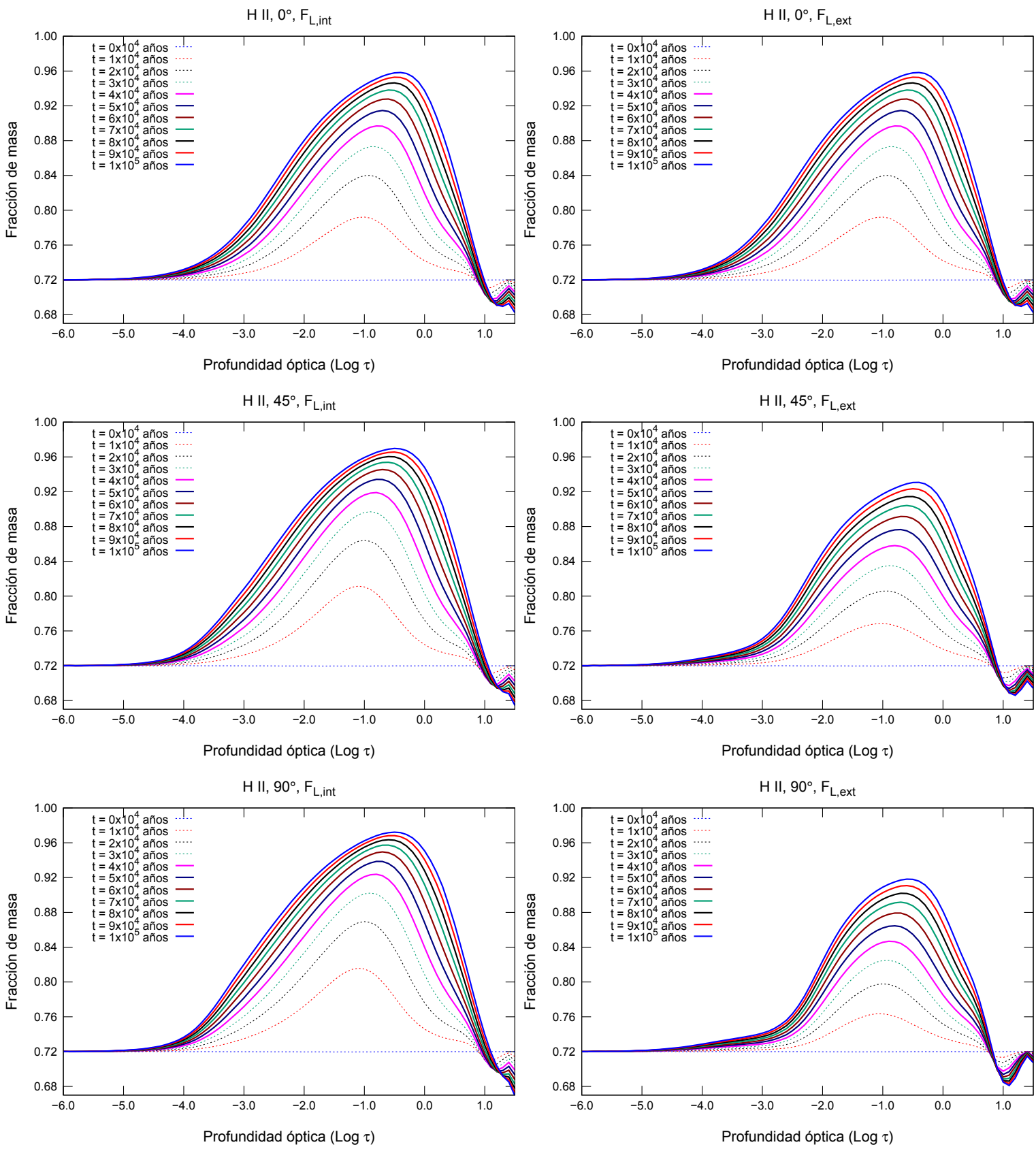

Figura 9.3: Fracción de masa de H in para un campo magnético de 1000 Gauss en función de la profundidad ópitca. Se muestran los resultados para latitudes correspondientes al polo, $45^{\circ}$, y al ecuador, a distintos tiempos (0 a 100000 años con $\Delta t=10000$ años).

En cada figura, todos los gráficos poseen la misma escala para poder compararlos entre sí. En todos los casos la intensidad del campo magnético es de 1000 Gauss. A la izquierda se ubican los modelos con la fuerza de Lorentz apuntando hacia el interior de la estrella, mientras que 
a la derecha figuran los correspondientes a la fuerza de Lorentz apuntando hacia afuera. En la parte superior mostramos los modelos correspondientes a un ángulo de latitud magnética $0^{\circ}$, es decir en los polos magnéticos. Se puede ver que en este caso los gráficos de la izquierda y de la derecha coinciden, ya que el comportamiento de la gravedad efectiva es igual al de un modelo sin un campo magnético. En los gráficos centrales, corresponden a modelos con una latitud magnética de $45^{\circ}$, y los de la parte inferior corresponden a modelos con una latitud magnética de $90^{\circ}$, es decir, sobre el ecuador magnético. En todos los casos graficamos las fracciones de masa para modelos desde 0 a 100000 años cada 10000 años.

Lo que notamos es que para todas las especies químicas analizadas, la difusión es mucho mayor cuando la fuerza de Lorentz está dirigida hacia el interior de la estrella. También vemos que la difusión es cada vez mayor al ir aumentando el ángulo de latitud magnética, es decir cuando nos acercamos al ecuador magnético. Todo lo contrario ocurre cuando la fuerza de Lorentz apunta hacia afuera, en donde la difusión es cada vez menor cuando nos acercamos al ecuador magnético. A tal punto, que en el caso del He I, en el ecuador y con la fuerza de Lorentz apuntando hacia afuera, casi no hay difusión.

En la figura 9.6. mostramos a la izquierda la fracción de masa de H II (arriba), He I (centro) y He II (abajo), en función de la profundidad óptica, considerando que la difusión estuvo actuando durante 100000 años. Para cada especie química se muestran las correspondientes fracciones de masa para latitudes magnéticas de $0^{\circ}, 36^{\circ}, 63^{\circ}$ y $90^{\circ}$. También se muestra para cada latitud, los casos correspondientes a la fuerza de Lorentz apuntando hacia el interior (líneas llenas) y hacia el exterior (líneas entrecortadas).

Para el H II (arriba), vemos que si la fuerza de Lorentz apunta hacia adentro, la fracción de masa para una dada profundidad óptica aumenta desde el polo al ecuador magnético. Esto se cumple para todas las profundidades ópticas. Todo lo contrario ocurre cuando la fuerza de Lorentz apunta hacia el exterior de la estrella. En el caso del He II (abajo), la fracción de masa aumenta para todas las profundidades ópticas al dirigirnos hacia el ecuador si la fuerza de Lorentz apunta hacia afuera. Lo contrario ocurre cuando la fuerza de Lorentz apunta hacia el interior. Distinto es el caso del He II, en donde, si la fuerza de Lorentz apunta hacia el interior, la fracción de masa aumenta hacia el ecuador magnético salvo para profundidades ópticas entre $\log \tau=-2.5$ y $\log \tau=-0.5$ en donde las líneas del gráfico se entrecruzan y la fracción de masa disminuye hacia el ecuador magnético. Como en los demás casos, el comportamiento es inverso cuando la fuerza de Lorentz apunta en el sentido opuesto. Todo esto se ve más claramente en las figuras 9.7, 9.8,9.9 y 9.10 que comentaremos más adelante. En el lado derecho de la figura 9.6 mostramos el comportamiento del logaritmo del número de partículas en lugar de la fracción de masa. 

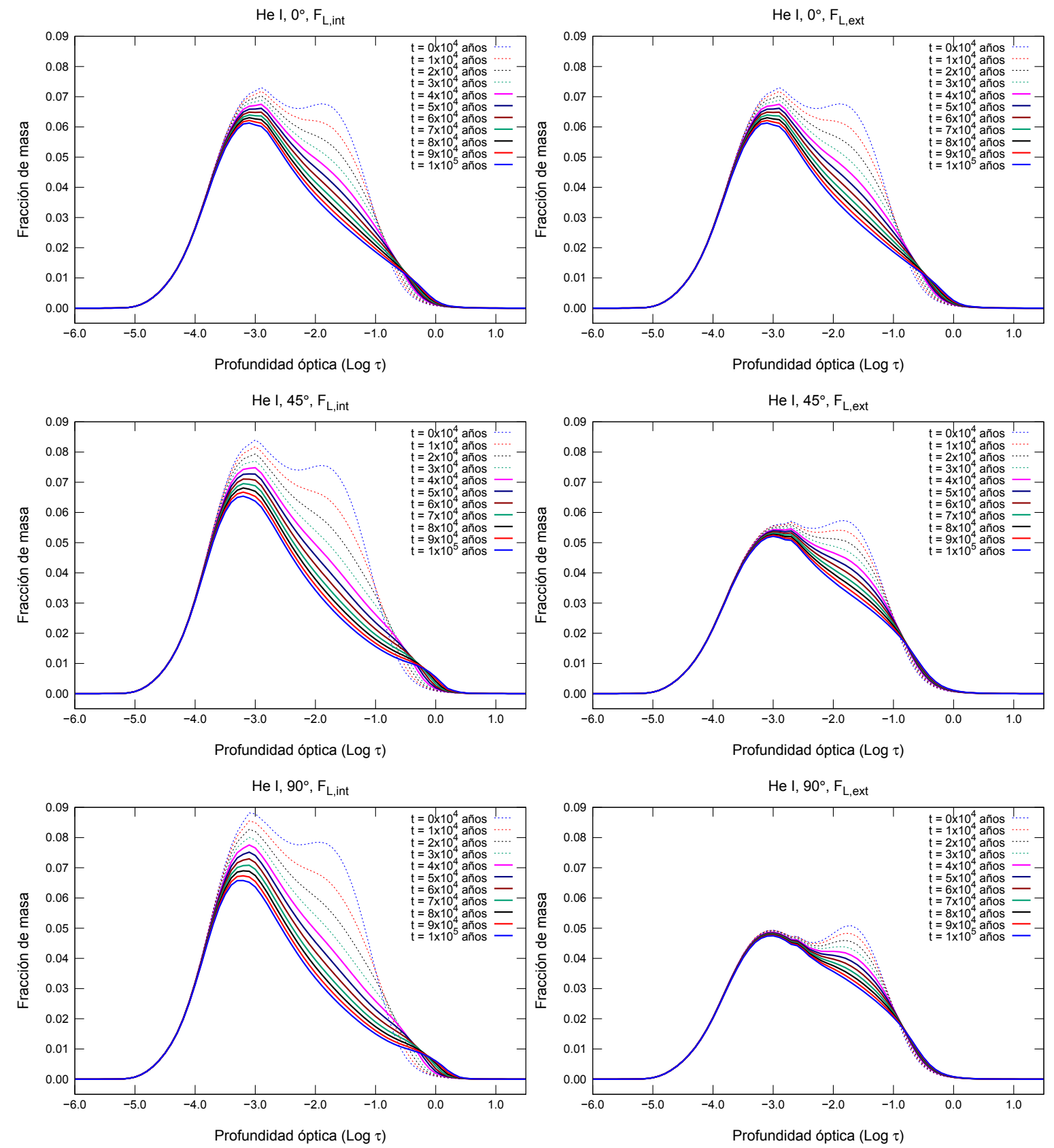

Figura 9.4: Fracción de masa de He I para un campo magnético de 1000 Gauss en función de la profundidad ópitca. Se muestran los resultados para latitudes correspondientes al polo, $45^{\circ}$, y al ecuador, a distintos tiempos (0 a 100000 años con $\Delta t=10000$ años). 

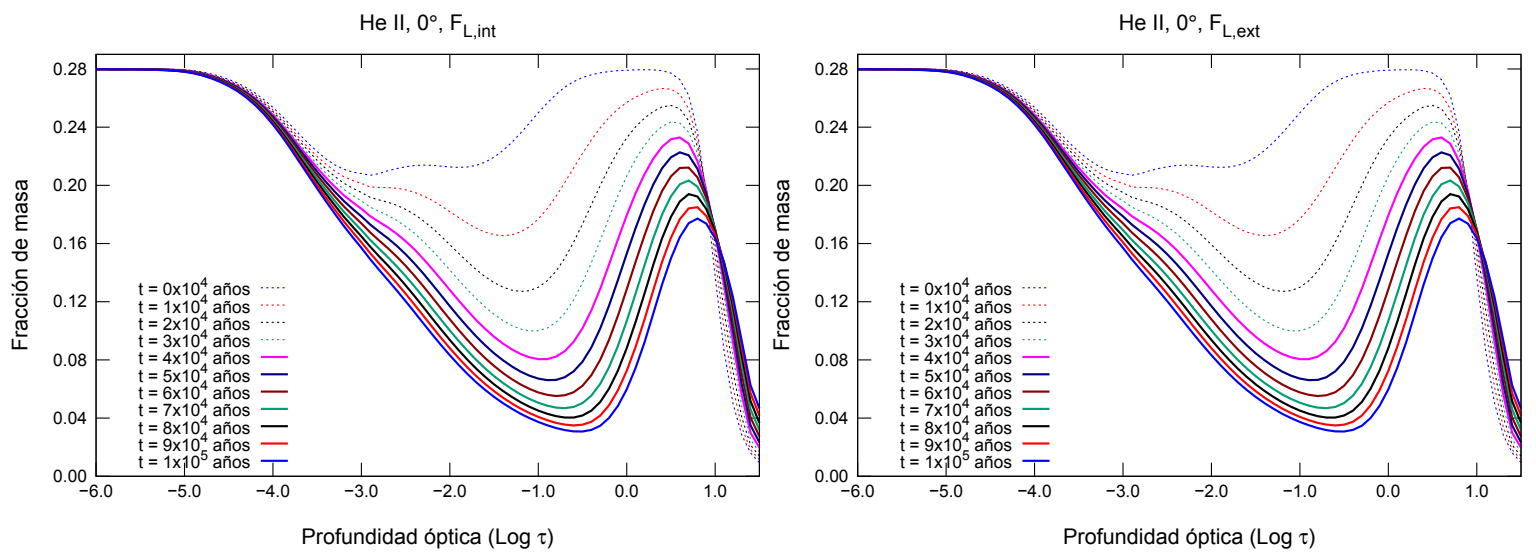

He II, $45^{\circ}, F_{L \text {,int }}$
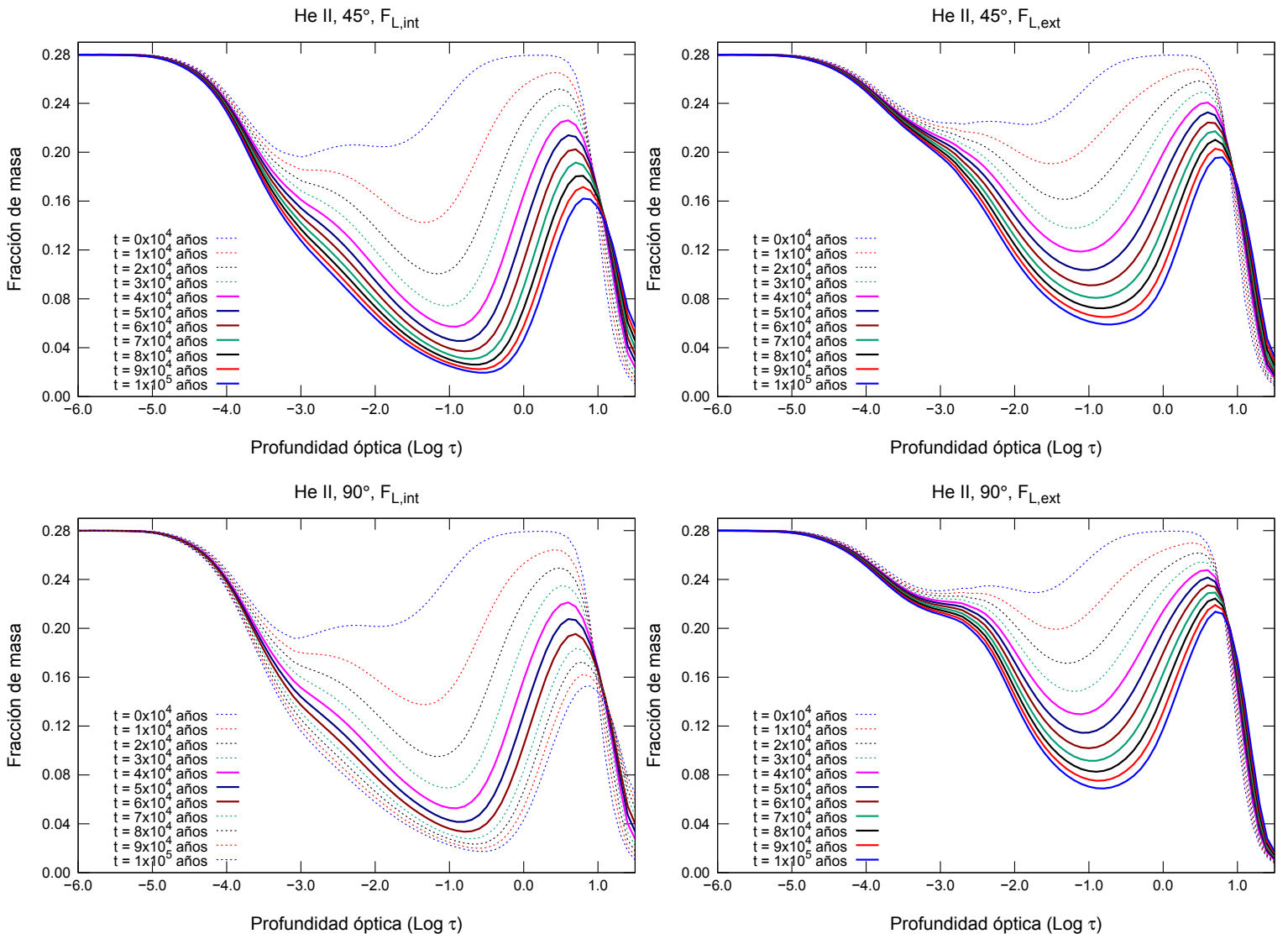

Figura 9.5: Fracción de masa de He II para un campo magnético de 1000 Gauss en función de la profundidad ópitca. Se muestran los resultados para latitudes correspondientes al polo, $45^{\circ}$, y al ecuador, a distintos tiempos (0 a 100000 años con $\Delta t=10000$ años). 

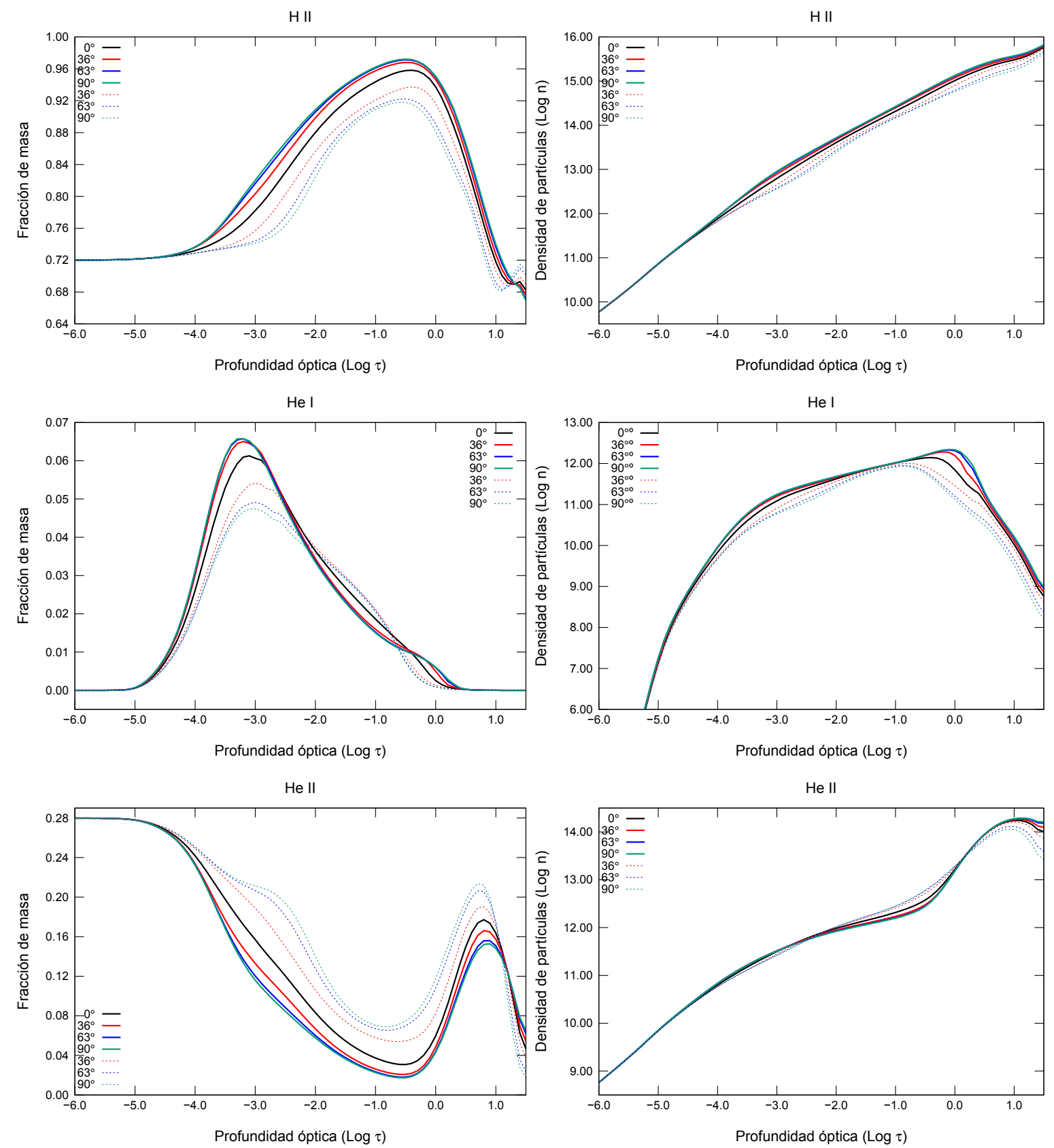

Figura 9.6: Fracción de masa (izquierda) y densidad de partículas (derecha), de H II (arriba), He I (centro) y He II (abajo), en función de $\log \tau$, para distintas latitudes y para direcciones opuestas de la fuerza de Lorentz luego de 100000 años de procesos difusivos. 
Para visualizar mejor la distribución de las abundancias de las distintas especies químicas después de los procesos de difusión, en las figuras 9.7, 9.8, 9.9 y 9.10, mostramos en un disco que representa el hemisferio visible de la estrella y en degradé de colores las fracciones de masa de las distintas especies. La elección de la profundidad óptica en la que se muestra la distribución de abundancias es arbitraria, eligiendo las que muestran un comportamiento general. Sólo en el caso de la distribución de He II, mostramos dos profundidades ópticas distintas para ejemplificar comportamientos distintos. En todos los casos se trata del modelo correspondiente a una estrella $\mathrm{B} 2 \mathrm{~V}$, con una abundancia inicial de tipo solar y para una intensidad del campo magnético de 1000 Gauss. También se muestra la distribución de abundancias para los dos sentidos de la fuerza de Lorentz. La escala de colores para la fracción de masa es distinta para cada gráfico ya que de considerar las mismas escalas se pierden los detalles. Como regla general, los colores rojos corresponden a una menor fracción de masa, mientras que los tonos violetas corresponden a una fracción de masa mayor.
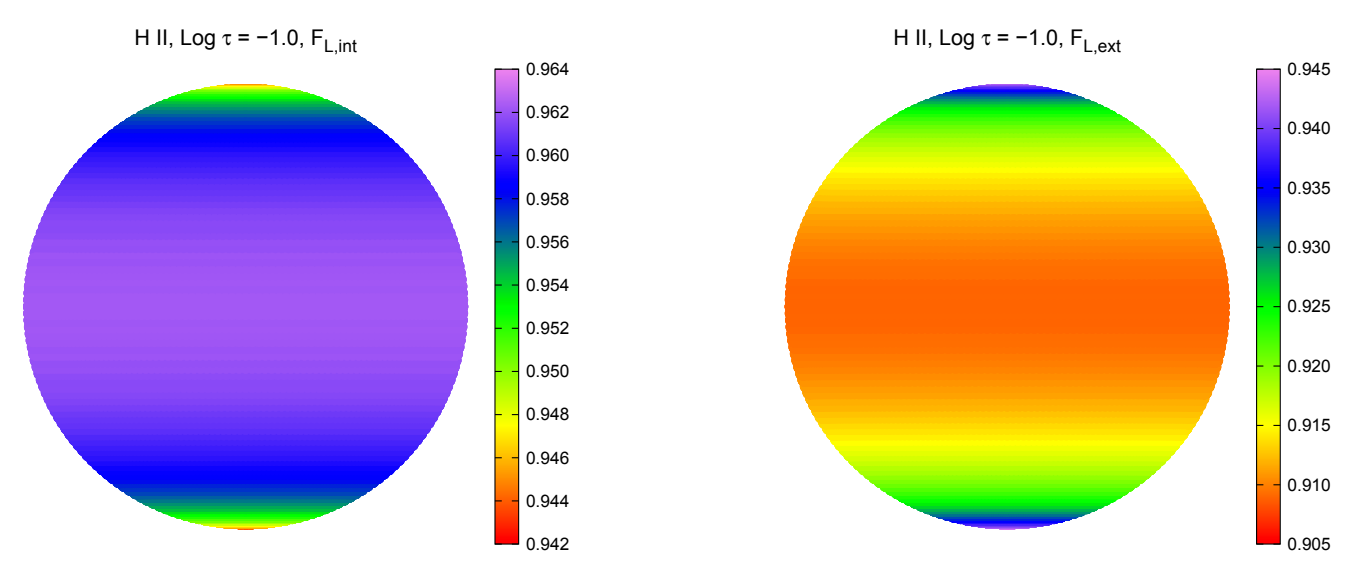

Figura 9.7: Distribución de la fracción de masa con la latitud de H II, en $\log \tau=-1.0$, para un campo magnético de 1000 Gauss, con la $F_{L}$ apuntando hacia el interior (izquierda) y apuntando hacia el exterior (derecha).

En la figura 9.7 vemos la distribución de $\mathrm{H}$ II con la latitud magnética, a una profundidad óptica de $\log \tau=-1.0$. El gráfico de la izquierda corresponde a un modelo con la fuerza de Lorentz dirigida hacia el interior, mientras que el de la derecha corresponde al caso opuesto. En el primer gráfico vemos que la fracción de masa de H II es mayor en el ecuador que en los polos, mientras que ocurre lo contrario ocurre cuando la fuerza de Lorentz se dirige hacia el exterior.

La otra especie química bastante abundante en estos modelos es el He II, cuya distribución mostramos en la figura 9.8 también en $\log \tau=-1.0$. Como era de esperarse, tenemos el resultado opuesto al $\mathrm{H}$. Cuando la fuerza de Lorentz apunta hacia el interior, la fracción de masa de He II es mayor en los polos que en el ecuador, al revés de lo que ocurría con el H II. Inversamente, cuando la fuerza de Lorentz apunta hacia el interior, la fracción de masa de 

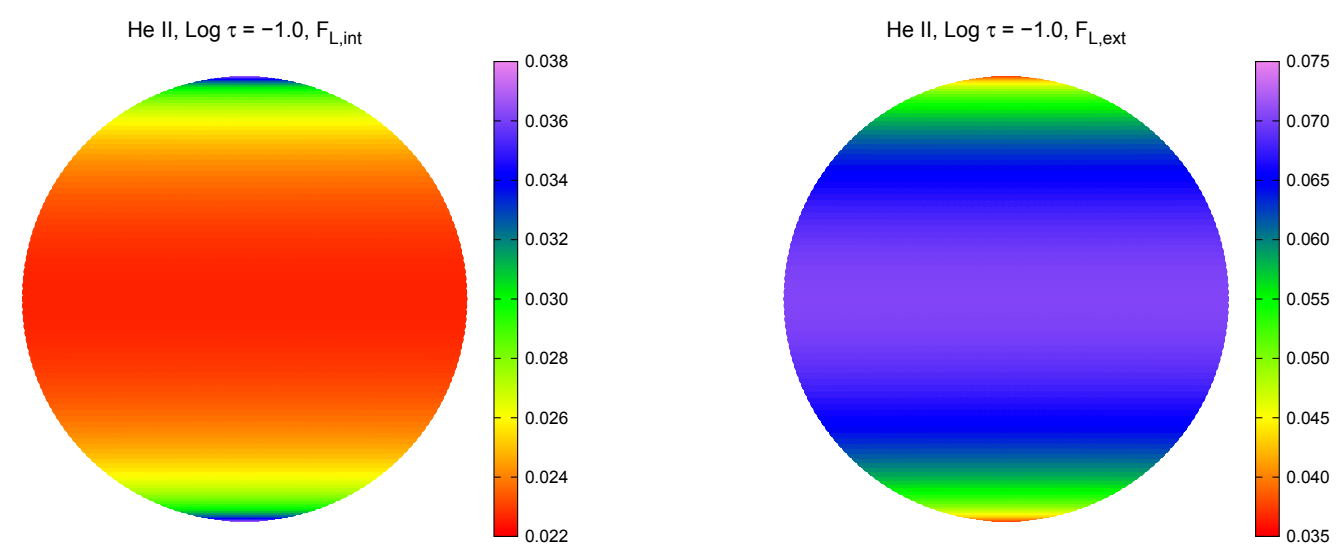

Figura 9.8: Distribución de la fracción de masa con la latitud de He II, en $\log \tau=-1.0$, para un campo magnético de 1000 Gauss, con la $F_{L}$ apuntando hacia el interior (izquierda) y apuntando hacia el exterior (derecha).

He II es mayor en el ecuador.

En el caso de He I, es mucho menos abundante que en los dos ejemplos anteriores, vemos que el comportamiento cambia con la profundidad óptica. En las figuras 9.9 y 9.10 . mostramos el comportamiento para dos profundidades ópticas, $\log \tau=-0.2$ y $\log \tau=-1.0$, respectivamente.
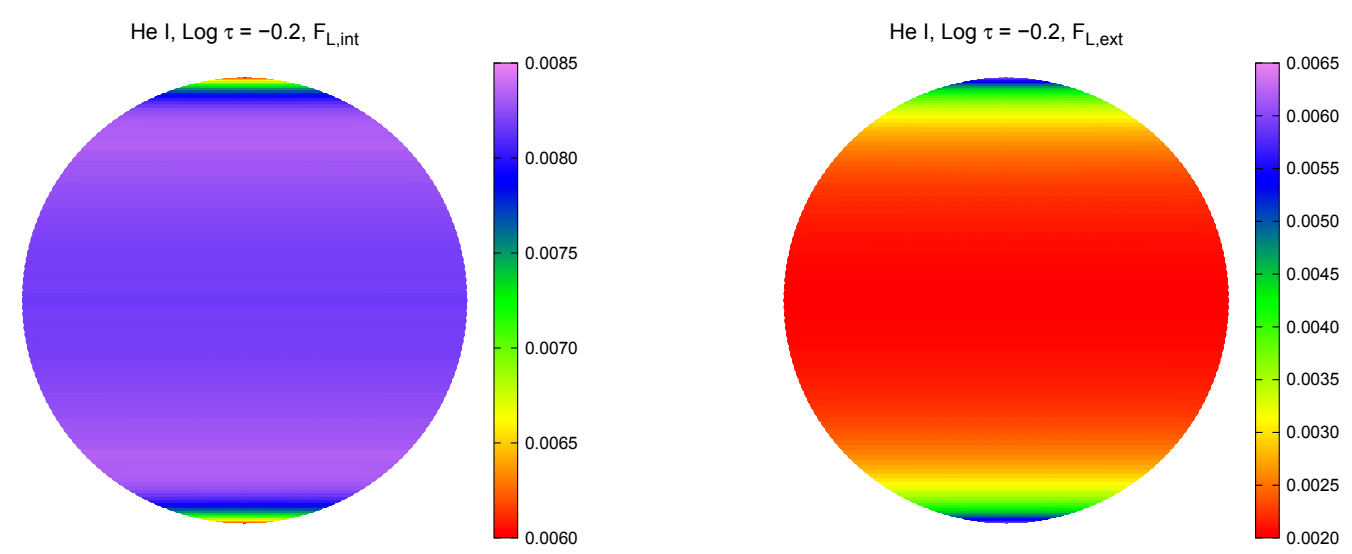

Figura 9.9: Distribución de la fracción de masa con la latitud de He I, en $\log \tau=-0.2$, para un campo magnético de 1000 Gauss, con la $F_{L}$ apuntando hacia el interior (izquierda) y apuntando hacia el exterior (derecha).

Vemos que mientras para $\log \tau=-0.2$ la fracción de masa es mayor en los polos cuando la fuerza de Lorentz apunta hacia afuera, en $\log \tau=-1.0$, la fracción mayor en los polos cuando apunta hacia adentro. Ahora bien, cuando vemos qué ocurre cuando la fuerza de Lorentz apunta hacia afuera en $\log \tau=-1.0$, no vemos que la fracción de masa sea máxima en el ecuador, sino que lo es en latitudes por encima y por debajo del mismo. Lo mismo ocurre en $\log \tau=-0.2$, pero cuando la fuerza de Lorentz apunta hacia adentro. En este último caso, 
las diferentes zonas no son tan marcadas. En otras profundidades ópticas tendremos que la fracción de masa es máxima en el ecuador.
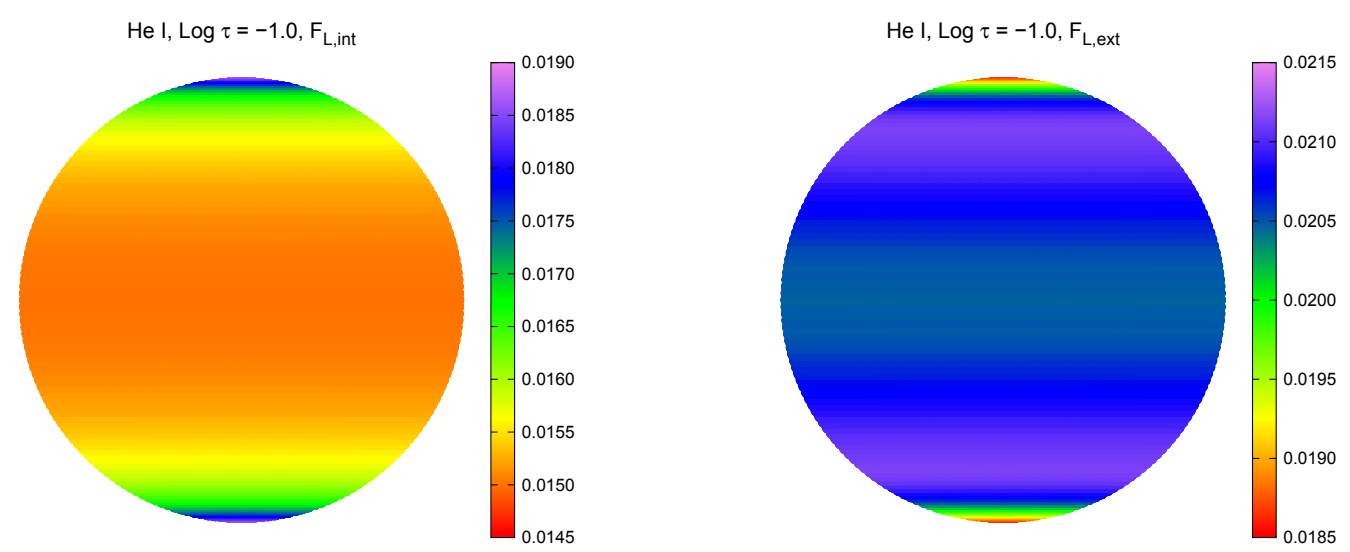

Figura 9.10: Distribución de la fracción de masa con la latitud de He I, en $\log \tau=-1.0$, para un campo magnético de 1000 Gauss, con la $F_{L}$ apuntando hacia el interior (izquierda) y apuntando hacia el exterior (derecha).

Por otro lado, a continuación analizaremos que sucede con los perfiles de línea después que la difusión ha alcanzado el equilibrio. Puntualmente veremos como han sido afectados los anchos equivalentes. En la figura 9.11 mostramos los anchos equivalentes de las líneas He I $\lambda 4026$ (izquierda) y He I $\lambda 5875$ (derecha). En rojo se muestran los anchos equivalentes antes de la difusión y en azul los obtenidos después de alcanzado el equilibrio.
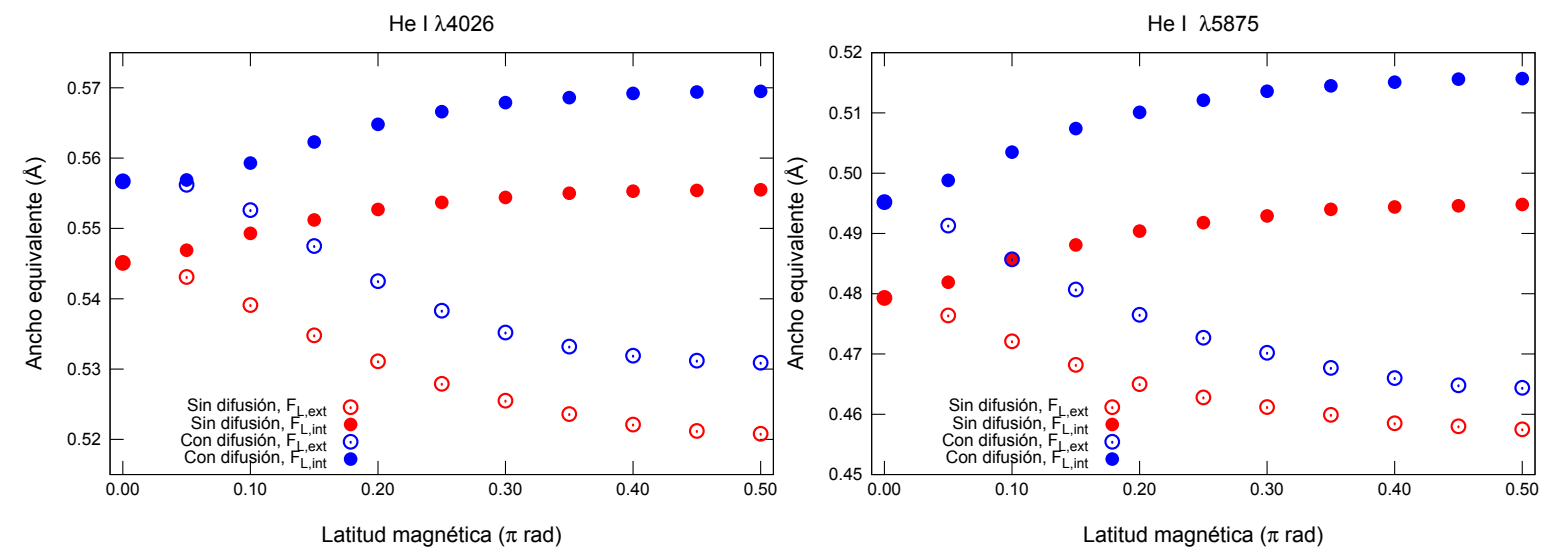

Figura 9.11: Anchos equivalentes de He I $\lambda 4026$ (izquierda) y He I $\lambda 5875$ (derecha) con la fuerza de Lorentz en sentidos opuestos, antes y después de que actuó la difusión.

Lo primero que notamos y que se repite en las otras líneas es que para una dada latitud magnética, el ancho equivalente será mayor después que la difusión ha tenido lugar. Esto también ocurre con modelos de tipos espectrales más tardíos.

Para He I $\lambda 4026$, la variación del ancho equivalente entre el polo magnético y el ecuador 
es del $4.5 \%$ si la fuerza de Lorentz apunta hacia el exterior, mientras que es de un $1.9 \%$ si la fuerza de Lorentz apunta hacia el interior. Después de que la difusión ha actuado, esos valores se incrementan levemente, hasta llegar a $4.6 \%$ y $2.3 \%$, respectivamente. En el caso de He I $\lambda 5875$, las variaciones son mayores, de $4.5 \%$ y $3.2 \%$ originariamente, a $6.2 \%$ y $4.3 \%$ después. No las hemos graficado aquí, pero para He i $\lambda 4921$ las variaciones son de un $2 \%$ más en ambas direcciones de la fuerza de Lorentz después de la difusión. En el caso de He I $\lambda 5875$, las diferencias entre las regiones consideradas disminuye, mientras que para He I $\lambda 4471$, disminuyen cuando la fuerza de Lorentz apunta hacia el exterior y aumenta cuando apunta en sentido contrario, aunque en ambos casos las variaciones son pequeñas.

\subsection{Efectos de las distintas intensidades de los campos magnéticos sobre la difusión}

A continuación, analizaremos los efectos sobre la difusión con distintas intensidades del campo magnético y determinaremos si existe alguna relación o no con las regiones en donde ocurre la difusión y la intensidad de los campos magnéticos o si hay una dependencia de cuánto se difunde algún elemento químico con la intensidad de los campos.

En la figura 9.12 graficamos la fracción de masa de H II (arriba), He I (centro) y He II (abajo) en función de la profundidad óptica. A la izquierda mostramos los modelos correspondientes a la fuerza de Lorentz apuntando hacia el interior, mientras que en los de la derecha la fuerza apunta hacia el exterior. En líneas entrecortadas se muestran las fracciones de masa antes de difundir, y en trazo lleno las fracciones de masa obtenidas después de difundir. Se muestran modelos con intensidades del campo de 10, 20, 500, 1000 y 2000 Gauss.

En líneas generales vemos que los comportamientos son similares para todas las distintas intensidades, aunque no exactamente iguales, es decir que los elementos se difunden prácticamente en las mismas regiones y de la misma forma.

Como regla general, vemos que para todas las intensidades, la difusión es mayor cuando la fuerza de Lorentz apunta hacia adentro. También vemos que las diferencias en la distribución de las abundancias de los distintos elementos con los campos magnéticos antes de difundir, se hacen más notorias después de que los procesos de difusión alcanzaron el equilibrio. Incluso, son mayores cuando la fuerza de Lorentz apunta hacia el exterior y se ve que no tienen una tendencia con la intensidad del campo cómo sí ocurre en el caso de la fuerza de Lorentz apuntando hacia el interior. Esto significa, que en el caso de la fuerza de Lorentz apuntando hacia el exterior, las líneas que identifican a cada intensidad de campo magnético se entrecruzan, mientras que en el caso en que apunta hacia el interior, aparecen más ordenadas, sin cruzarse. 
H II, $90^{\circ}, F_{L, \text { int }}$

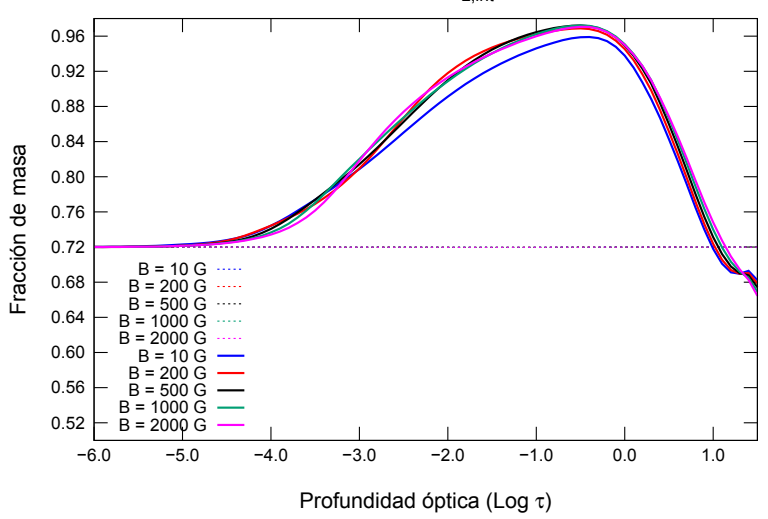

$\mathrm{He}$ I, $90^{\circ}, \mathrm{F}_{\mathrm{L} \text {,int }}$

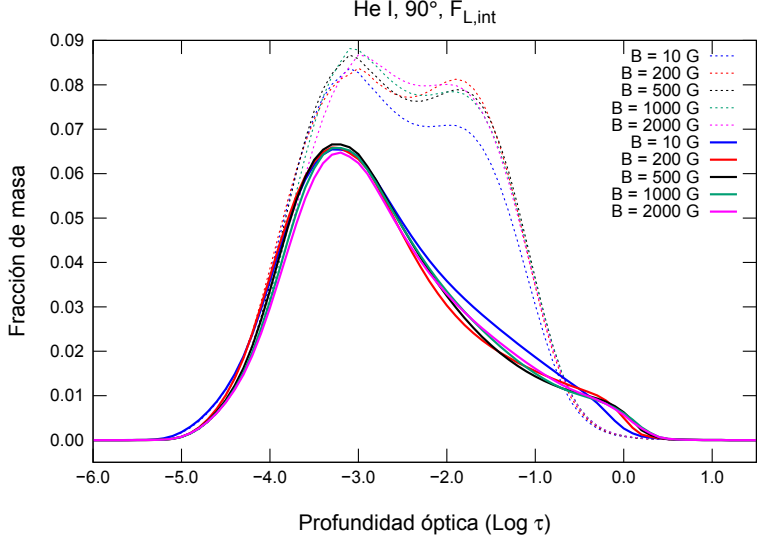

He II, $90^{\circ}, F_{L, \text { int }}$

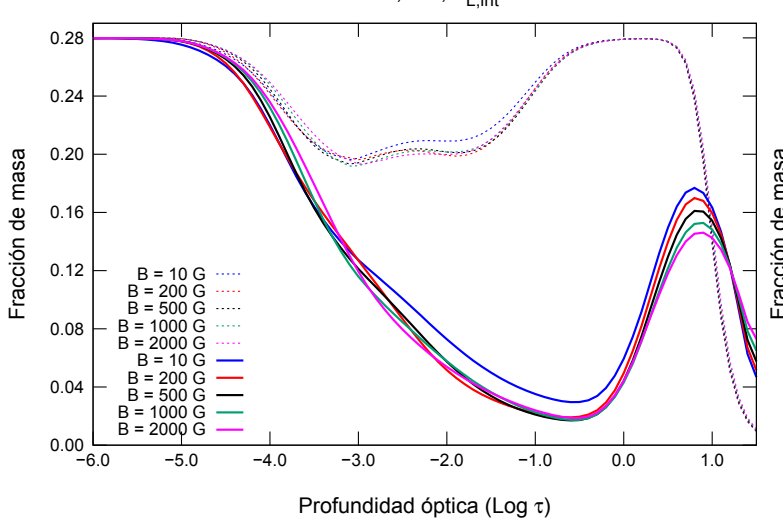

$\mathrm{HII}, 90^{\circ}, \mathrm{F}_{\mathrm{L}, \text { ext }}$

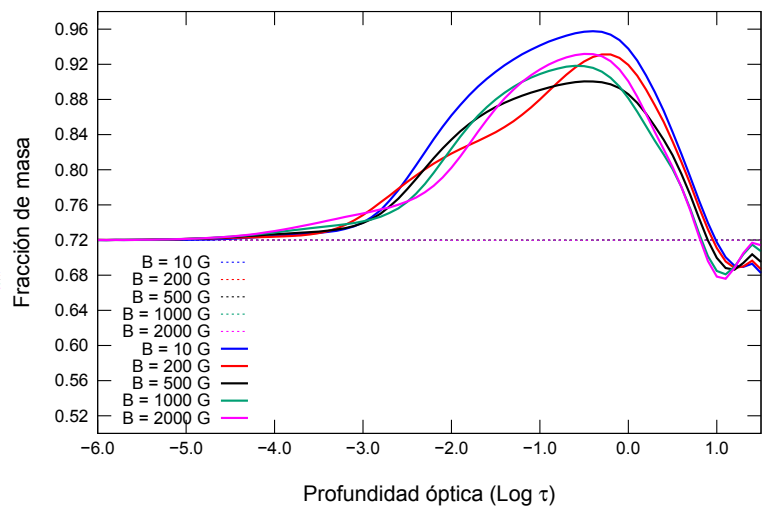

$\mathrm{He}$ I, $90^{\circ}, \mathrm{F}_{\mathrm{L}, \mathrm{ext}}$
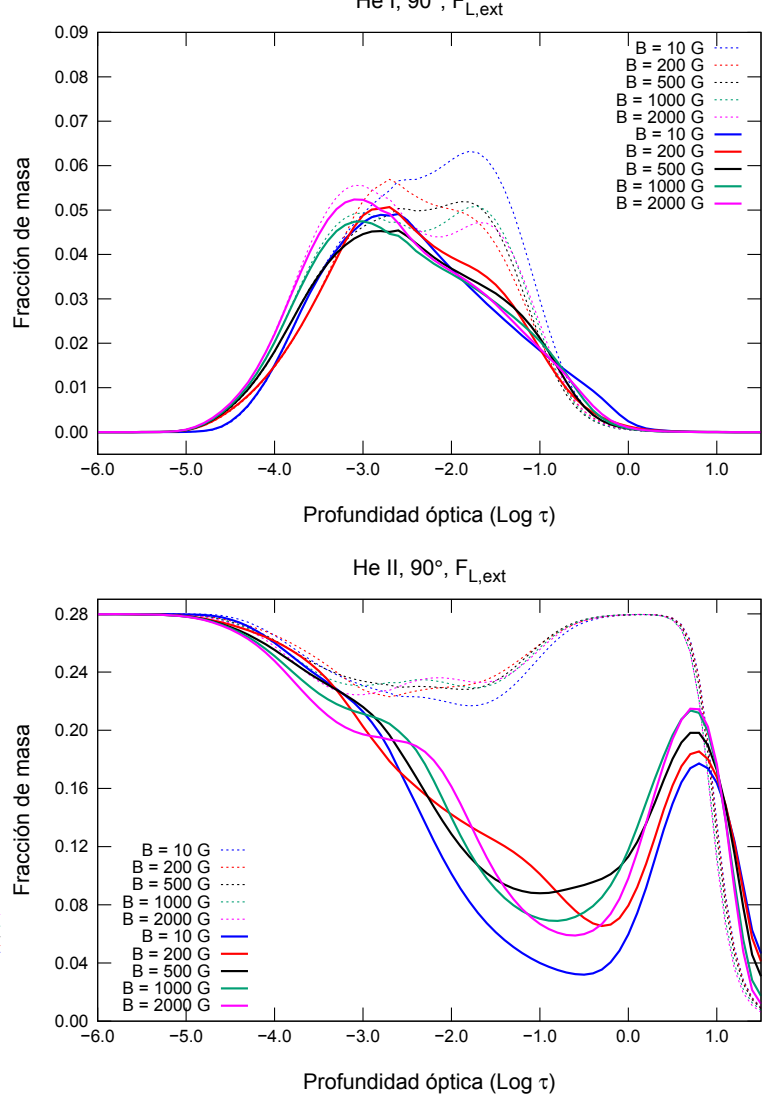

Figura 9.12: Fracción de masa, H II (arriba), He I (centro) y He II (abajo), para distintas intensidades del campo magnético, con $F_{L}$ hacia el interior (izquierda) y hacia el exterior (derecha). Todos los gráficos corresponden al ecuador magnético. En trazo punteado, corresponde a antes de difundir y en trazo sólido, después de difundir. 


\subsection{Difusión en estrellas de tipo espectral B5V}

Consideraremos ahora nuevamente el caso de una estrella de menor temperatura, como lo son las estrellas de tipo espectral B5V. No mostraremos aquí un análisis tan exhaustivo como el realizado anteriormente con los modelos de una estrella $\mathrm{B} 2 \mathrm{~V}$. Al igual que lo hicimos en la sección $\$ 5.3$. consideramos una temperatura de $15200 \mathrm{~K}$, abundancia de tipo solar y una intensidad del campo magnético de 1000 Gauss.

Una diferencia respecto de las B2V es que el tiempo de convergencia de la difusión es mucho más corto. Se llega al equilibrio en unos 50000 años. En la figura 9.13, mostramos la fracción de masa después de 50000 años para cuatro especies químicas distintas, para un modelo sin campo magnético.

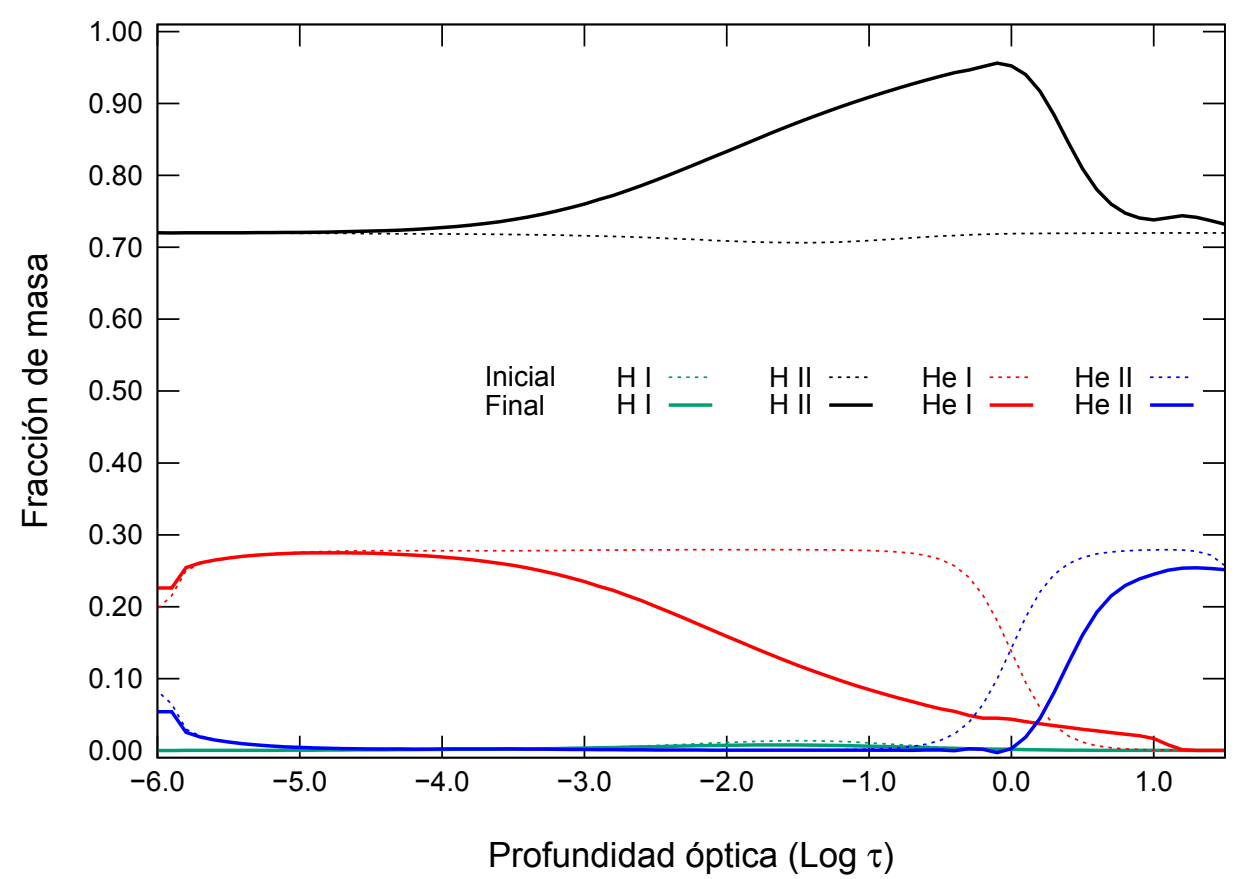

Figura 9.13: Fracción de masa de las distintas especies químicas en función de la profundidad óptica de Rosseland $(\log \tau)$. Los trazos llenos corresponden a las fracciones de masa iniciales y los entrecortados a las fracciones de masa después de 50000 años. Las especies graficadas son H I (verde), H iI (negro), He I (rojo) y He II (azul).

La otra diferencia es la abundancia de He i inicial, que casi no se encontraba presente en los modelos anteriores, y el He II, sólo presente en las capas más internas.

En los gráficos de la figura 9.14, mostramos con más detalle la evolución temporal de las fracciones de masa para las cuatro especies químicas analizadas. Vemos que en el caso del $\mathrm{H}$ neutro (poco abundante), va disminuyendo su fracción de masa con el tiempo, mientras que el $\mathrm{H}$ ionizado la aumenta, fundamentalmente en la región de $\log \tau=0$. En el caso del He neutro, la fracción de masa decae en una gran región, permaneciendo invariante en 
las regiones más externas, y aumentando algo en las capa mas internas. En cuanto al He ionizado una vez, en las regiones en donde se encuentra presente, disminuye su abundancia fraccional.
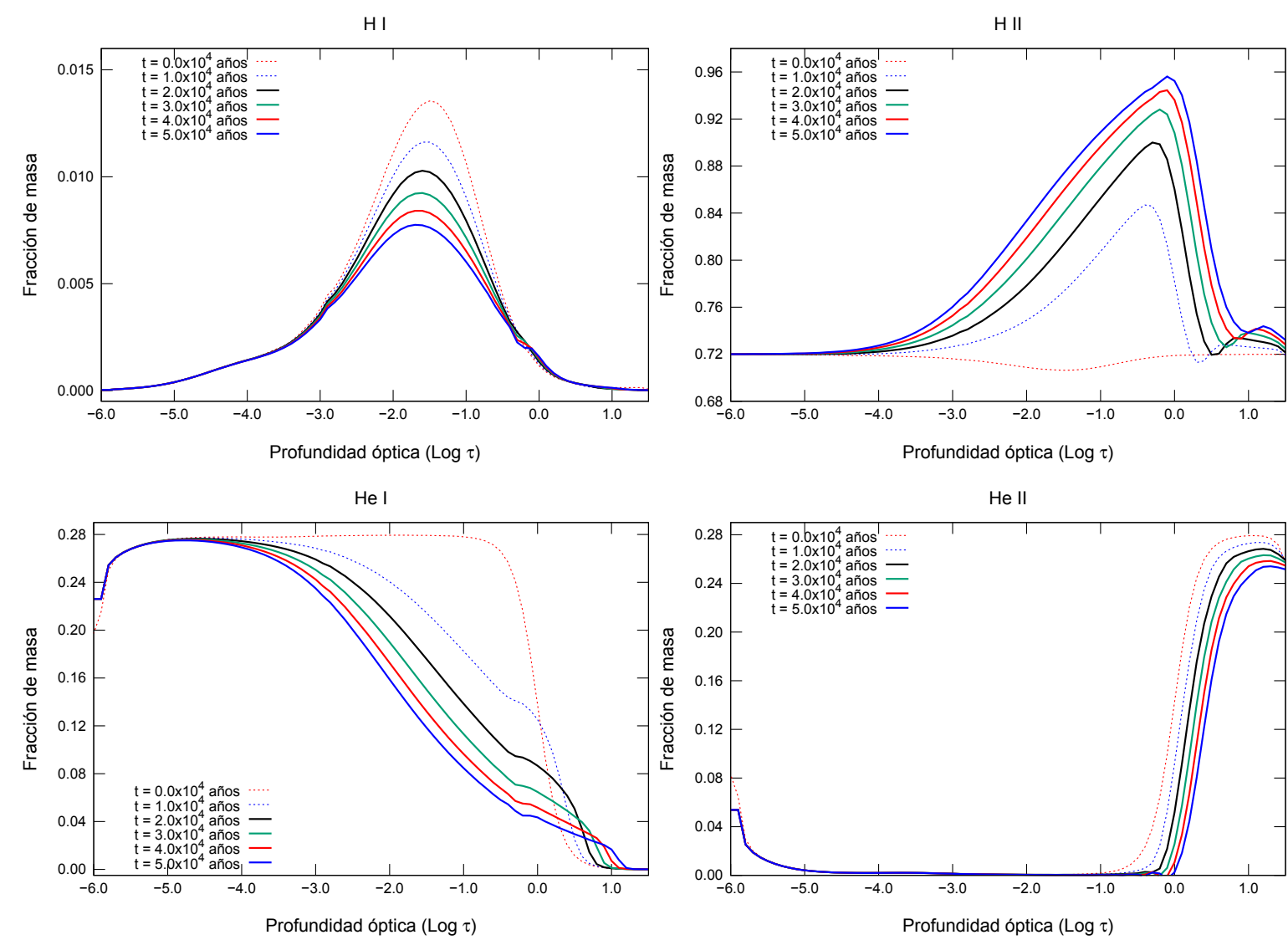

Figura 9.14: Fracción de masa de las distintas especies químicas en función de la profundidad óptica de Rosseland $(\log \tau)$, para distintos tiempos. Arriba izquierda: H I. Arriba derecha: H II. Abajo izquierda: He I. Abajo derecha: He II.

En los gráficos de las figuras 9.15 y 9.16 mostramos la distribución de la fracción de masa con la latitud de H II y He I, respectivamente, las dos especies más abundantes en estos modelos. En ambos casos, mostramos las fracciones de masa a una profundidad óptica de $\log \tau=-1.0$, y con la fuerza de Lorentz en sentidos opuestos.

Podemos observar que el comportamiento es el mismo que encontramos en el caso de modelos de estrellas B2V. Vemos que si la especie química tiene una fracción de masa mayor en el ecuador que en los polos magnéticos, entonces, si la fuerza de Lorentz apunta en sentido opuesto, la fracción de masa de esa especie será menor en el ecuador. También, como era de esperarse, vemos que en las regiones donde la fracción de masa $\mathrm{H}$ aumenta, la de He disminuye y viceversa. En estos modelos, no hemos encontrado para ninguna especie química, ni profundidad óptica que el máximo de la fracción de masa se encuentre en latitudes intermedias. 

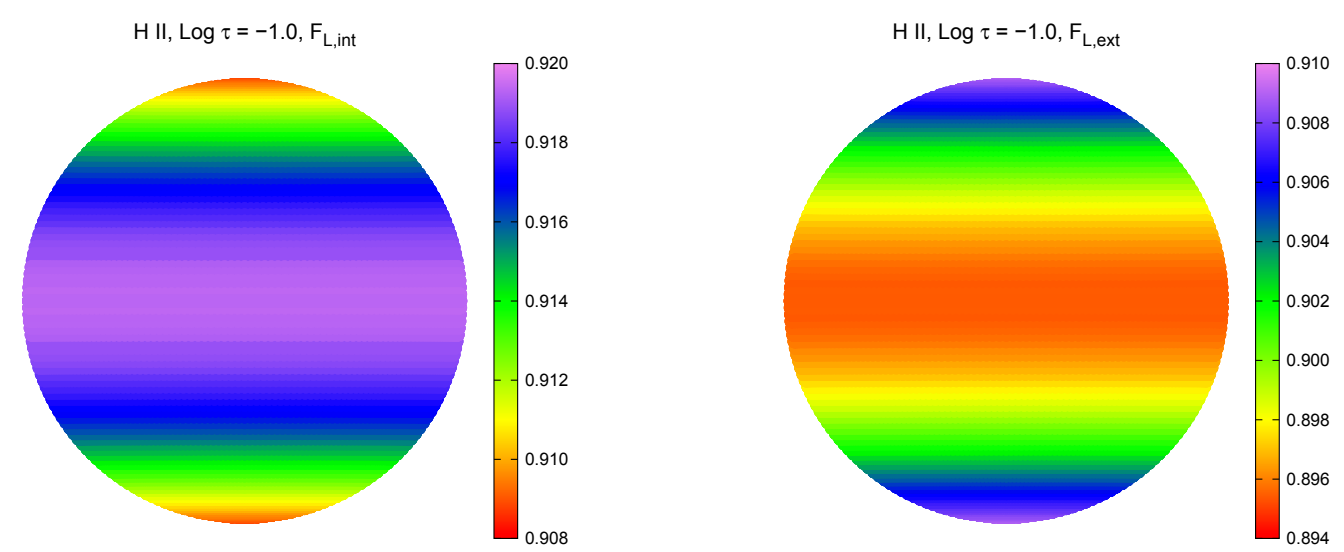

Figura 9.15: Distribución de la fracción de masa con la latitud de H II, en $\log \tau=-1.0$, para un campo magnético de 1000 Gauss, con la $F_{L}$ apuntando hacia el interior (izquierda) y apuntando hacia el exterior (derecha).
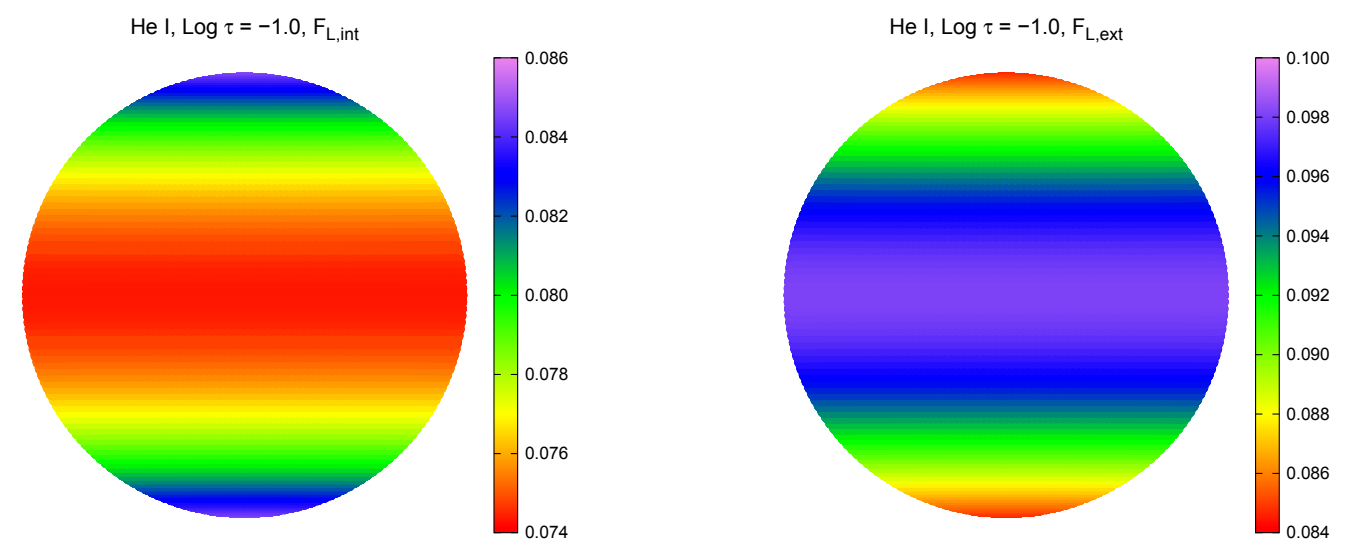

Figura 9.16: Distribución de la fracción de masa con la latitud de H II, en $\log \tau=-1.0$, para un campo magnético de 1000 Gauss, con la $F_{L}$ apuntando hacia el interior (izquierda) y apuntando hacia el exterior (derecha).

Para completar nuestro análisis, a continuación vemos qué sucede con los perfiles de las líneas de He. A modo de ejemplo, en la figura 9.17 mostramos los anchos equivalentes en función de la latitud magnética de He i $\lambda 4026$ (izquierda) y He I $\lambda 4921$ (derecha). En ambos casos, mostramos los anchos equivalentes con la fuerza de Lorentz en sentidos opuestos para un campo magnético con una intensidad de 1000 Gauss, antes de que actuara la difusión (rojo) y después de que haya alcanzado el equilibrio (azul).

A diferencia de lo que ocurría en los modelos de estrellas de tipo espectral B2V, si bien las líneas son más intensas cuando la fuerza de Lorentz apunta hacia el exterior, los anchos equivalentes son mayores al ir del polo al ecuador magnético. En las B2V, el ancho equivalente disminuye hacia el ecuador magnético si la fuerza de Lorentz apunta hacia afuera.

Para He I $\lambda 4026$, encontramos que antes de actuar la difusión la variación del ancho equivalente entre el polo y el ecuador magnético cuando la fuerza de Lorentz apunta al exterior es 

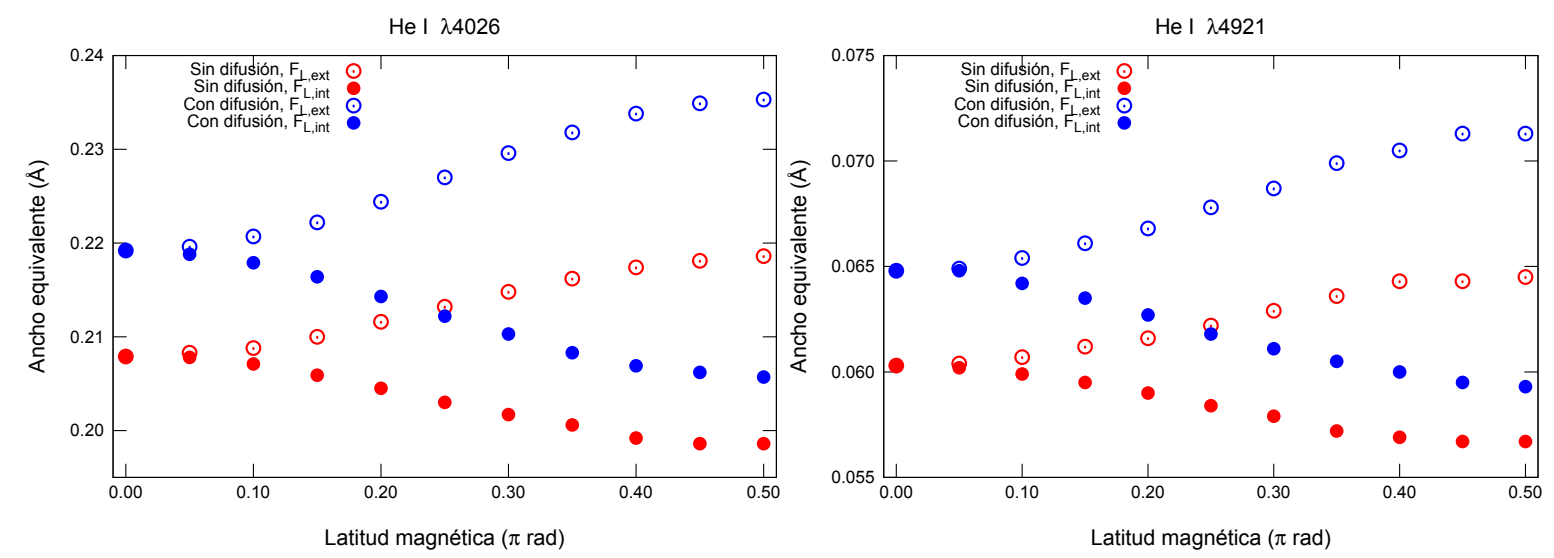

Figura 9.17: Anchos equivalentes de He I $\lambda 4026$ (izquierda) y He I $\lambda 4921$ (derecha) con la fuerza de Lorentz en sentidos opuestos, antes y después de que actuó la difusión.

del $5.1 \%$ y del $4.5 \%$ cuando apunta en sentido opuesto. Después que los procesos difusivos alcanzaron el equilibrio, encontramos que las variaciones son de $7.3 \%$ y $6.2 \%$, respectivamente. En el caso He I $\lambda 4471$, encontramos valores similares. Para He I $\lambda 4921$, variaciones son mayores, de $7.0 \%$ y $6.0 \%$ antes de la difusión a $10.0 \%$ y $8.5 \%$ después del equilibrio.

Para otras líneas las variaciones son menores en algunos casos o mayores en otros, pero siempre las diferencias entre el ecuador y los polos magnéticos es mayor después de que ha actuado la difusión.
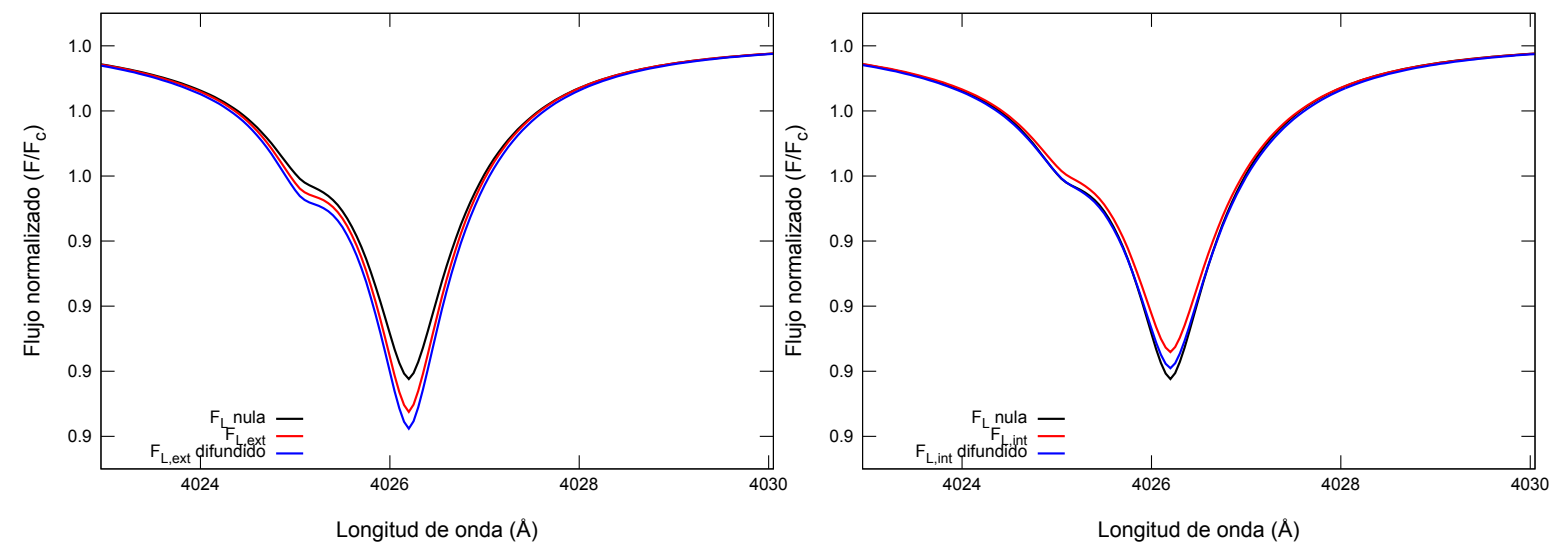

Figura 9.18: Perfiles de línea de He I $\lambda 4026$ (izquierda) con la fuerza de Lorentz hacia el exterior (izquierda) y hacia el interior (derecha). En ambos casos se muestra el perfil de la línea en ausencia de campo magnético (negro) y en presencia de un campo magnético de 1000 Gauss en el ecuador magnético, considerando procesos de difusión (azul) y sin ellos (rojo)

A modo de ejemplo, en la figura 9.18, mostramos el perfil de línea de He I $\lambda 4026$. El gráfico de la izquierda corresponde a modelos con la fuerza de Lorentz apuntando hacia el exterior de la estrella, mientras que el de la derecha se corresponde con modelos con la fuerza de Lorentz apuntando hacia el interior. Los perfiles en negro, corresponden a modelos sin 
campo magnéticos, mientras que los perfiles en rojo corresponden a modelos en el ecuador magnético con campos de 1000 Gauss de intensidad, sin que hayan tenido lugar los procesos de difusión. Por últimos, los perfiles en azul, corresponden a modelos con campo magnéticos en donde la difusión ha alcanzado el equilibrio. El cambio más importante ocurre cuando la fuerza de Lorentz apunta al exterior de la estrella. En ese caso vemos que la intensidad de la ĺinea aumenta al considerar la presencia de campos magnéticos, pero ese aumento es mayor si los procesos de difusión han ocurrido y alcanzado el equelibrio. Por otro lado, cuando la fuerza de Lorentz apunta hacia el interior, los campos magnéticos hacen que la intensidad de la línea disminuya, pero en este caso, si tenenmos difusión la disminución es menor. 


\section{Capítulo 10}

\section{Conclusiones}

Como mencionamos a lo largo de este trabajo, las estrellas químicamente peculiares son rotadores lentos y presentan intensificaciones o deficiencias de algunas especies químicas en sus atmósferas, en comparación con las características que presentan las estrellas enanas normales de la misma temperatura efectiva y se piensa que las anomalías químicas están causadas por fenómenos tales como la difusión y la presencia de campos magnéticos.

En particular nos interesó estudiar las estrellas peculiares en He, tanto las He-weak como las He-strong, las cuales presentan campos magnéticos. Para ello consideraremos el modelo de rotador magnético oblicuo donde la geometría del campo magnético la supusimos dipolar.

Analizamos la influencia de un campo magnético sobre la estructura de la atmósfera y su impacto sobre los perfiles de las líneas de He en estrellas peculiares en He, modelando fotosferas en equilibrio hidrostático, para estrellas de tipos espectrales desde B9 a B0 en donde se incluyó un término que contiene la fuerza de Lorentz.

Discutimos, los efectos locales sobre las líneas de He en presencia de campos magnéticos y analizamos sus variaciones para distintas latitudes magnéticas y también estudiamos los efectos globales. Por último, analizamos el efecto del campo magnético sobre la difusión resolviendo las ecuaciones de difusión atómica en ausencia y presencia de un campo magnético en una atmósfera estelar, y determinamos el perfil químico resultante con la latitud magnética para luego analizar los efectos locales.

En primer lugar, pudimos apreciar que la presencia de campos magnéticos produce cambios en la estructura interna de la atmósfera de una estrella y estos cambios se evidencian mayormente en la distribución de la presión del gas y su densidad.

Además, encontramos que la presencia de campos magnéticos provoca cambios en la estructura de la atmósfera local que dependen fuertemente de la latitud magnética. Esta ruptura 
en la simetría en latitud, sumado a considerar el modelo de rotador magnético oblicuo, permite la aparición de variaciones que coinciden con el período de rotación.

Encontramos que las variaciones dependen de la intensidad del campo magnético y estas se acrecientan cuando los campos son débiles. Las variaciones, fundamentalmente en el ancho equivalente e intensidad de las líneas, son mayores al ir aumentando la intensidad del campo magnético, hasta unos cientos de Gauss. Más allá de esas intensidades, las variaciones disminuyen levemente en su amplitud y luego están presentes pero permanecen casi invariantes ante el aumento de la intensidad de los campos, aún para intensidades de decenas de kilo Gauss.

También detectamos que las variaciones dependen de la dirección que posee la fuerza de Lorentz. En general los comportamientos son opuestos si el sentido de la fuerza de Lorentz cambia. Además, en casi todos los casos, las variaciones son más significativas cuando la fuerza de Lorentz apunta hacia el exterior de la estrella.

Fundamentalmente, encontramos que las variaciones de la estructura de la atmósfera se traducen en variaciones de los perfiles de las líneas, que son nuestro observable, en particular, en las de He que son las que nos interesan, tanto en su intensidad como en su forma.

Sabida es la dependencia de los perfiles de línea con la abundancia del correspondiente elemento químico. Nosotros encontramos que hay variaciones entre el polo y el ecuador magnético, y estas variaciones son mayores si la temperatura efectiva es menor, o si la abundancia de He es menor. De esta forma, para las variaciones locales, es decir para cada latitud magnética, encontramos que en modelos con abundancia solar, son más pequeñas que las observadas en las estrella peculiares de He. Por otro lado, si la abundancia de He es menor, encontramos que las variaciones son comparables a las observadas en las estrellas He-weak. Más aún, si consideramos modelos de menor temperatura efectiva, similares a una estrella B5V.

Al momento de considerar los efectos sobre todo el disco visible de la estrella, utilizamos la propuesta de Valyavin et al. 2004, que considera un campo de intensidad media que representa todas las contribuciones del campo magnético dipolar sobre todo el disco visible de la estrella y considerando una ley de oscurecimiento al borde. Este campo medio, y también la proyección de su componente angular latitudinal media, varían con la fase de rotación de la estrella. Hemos encontrado, que estos campos medios suavizan las variaciones locales, lo cual resultaría en que el efecto del campo magnético sobre las líneas sería menor.

Estas variaciones locales, y más aún las globales, no alcanzan para explicar las variaciones en las He-Strong y menos aún para las de a Cen. Por otro lado, no se deberían encontrar en nuestra galaxia estrellas jóvenes, como lo son las estrellas CP, que en realidad posean abundancias, homogéneamente distribuidas en la estrella, menores que la solar. Debido a 
que con nuestros modelos no podíamos explicar la existencia de zonas sobreabundantes o subabundantes de He, es que incluimos los procesos de difusión atómica en nuestros modelos. En cuanto a la difusión gravitatoria, encontramos que ante la presencia de campos magnéticos, la difusión es distinta en las regiones ecuatoriales que en las polares, con lo cual las diferencias entre las regiones se acentúan. También detectamos que prácticamente no hay cambios al considerar distintas intensidades del campo magnético. Sí hay cambios importantes dependiendo de la dirección de la fuerza de Lorentz, ya que en el caso de las especies químicas más abundantes, ésta determina si la correspondiente especie es más abundante en el ecuador o en el polo, para una misma capa (profundidad óptica). Esto es debido a que cuando la fuerza de Lorentz es mayor, los efectos de la difusión gravitatoria son mayores que cuando no existe campo magnético (en los polos), en cambio cuando la fuerza de Lorentz apunta en sentido opuesto, los efectos son menores, es decir que el campo magnético frena la convección. Si a partir de las observaciones podemos determinar las abundancias en estas regiones, entonces podríamos determinar la dirección de la fuerza de Lorentz.

También encontramos que en los casos estudiados, la difusión hace que la abundancia de He en las zonas de formación de las líneas de He disminuya, de forma tal que las líneas resultantes serían iguales a las que se formarían debido a una menor abundancia general de He.

De esta forma, los procesos de difusión explicarían la deficiencia anómala de He observada (o medida) en las estrellas He-Weak, mientras que las variaciones correlacionadas con la rotación, se deben a efectos magnéticos, acentuados por la difusión. Distinto es el caso de las He-Strong, en donde la magnitud de las sobreabundancias de He no pueden ser aparentemente explicadas por la difusión. Sabido es que la presión de radiación contribuye a la difusión selectiva de ciertos elementos como el Si generalmente sobreabundante en las estrellas CP de He, así como también el Mn y el Hg (Michaud, 1970). Por otro lado, si las velocidades de rotación son mayores que las observadas en estrellas $\mathrm{CP}$, aparecerían corrientes meridionales y turbulencias que inhibirían los procesos de difusión gravitatoria y esto es lo que les estaría sucediendo a las estrellas de abundancias normales.

La inclusión de la presión de radiación a los procesos difusivos, trabajo que se planea a futuro, debería contribuir a la formación de regiones sobreabundantes de He. Así mismo, en un trabajo anterior Vallverdú et al. 2007, encontramos que la presión de radiación afecta a la gravedad efectiva, en las regiones exteriores de la atmósfera, con lo cual agregaría un ingrediente más a la difusión. Además, hemos supuesto que el campo magnético, fósil en nuestro caso, es generado por un dipolo magnético, lo cual permitió romper la simetría en latitud. La utilización de geometrías cuadrupolares para el campo magnético también permitiría romper con la simetría azimutal. Otro hecho a tener en cuenta es que en realidad, 
la geometría de los campos magnéticos no es tan simple, ya que cada estrella presenta distribuciones superficiales distintas, y para un correcto modelado de estas estrellas, deberían considerarse modelos locales con campos magnéticos siguiendo la distribución superficial y posteriormente integrar todos los efectos sobre el hemisferio visible de la estrella.

Además, sería interesante realizar un modelo en donde de manera conjunta se modele el interior y la atmósfera. 


\section{Bibliografía}

Adelman, S. J. y Pyper, D. M.: 1985, Astron. Astrophys. Suppl. 62, 279

Alecian, E., Neiner, C., Wade, G. A., Mathis, S., Bohlender, D., Cébron, D., Folsom, C., Grunhut, J., Le Bouquin, J.-B., Petit, V., Sana, H., Tkachenko, A., y ud-Doula, A.: 2015, in G. Meynet, C. Georgy, J. Groh, y P. Stee (eds.), New Windows on Massive Stars, Vol. 307 of IAU Symposium, pp 330-335

Alecian, E., Wade, G. A., Catala, C., Bagnulo, S., Boehm, T., Bohlender, D., Bouret, J.-C., Donati, J.-F., Folsom, C. P., Grunhut, J., y Landstreet, J. D.: 2008, Astron. Astrophys 481, L99

Alecian, G. y Stift, M. J.: 2006, Astron. Astrophys 454, 571

Althaus, L. G. y Benvenuto, O. G.: 2000, Mon. Not. R. Astron. Soc. 317, 952

Althaus, L. G., Panei, J. A., Miller Bertolami, M. M., García-Berro, E., Córsico, A. H., Romero, A. D., Kepler, S. O., y Rohrmann, R. D.: 2009a, Astrophys. J. 704, 1605

Althaus, L. G., Panei, J. A., Romero, A. D., Rohrmann, R. D., Córsico, A. H., García-Berro, E., y Miller Bertolami, M. M.: 2009b, Astron. Astrophys 502, 207

Anderson, L. S.: 1989, Astrophys. J. 339, 558

Aurière, M., Wade, G. A., Silvester, J., Lignières, F., Bagnulo, S., Bale, K., Dintrans, B., Donati, J. F., Folsom, C. P., Gruberbauer, M., Hui Bon Hoa, A., Jeffers, S., Johnson, N., Landstreet, J. D., Lèbre, A., Lueftinger, T., Marsden, S., Mouillet, D., Naseri, S., Paletou, F., Petit, P., Power, J., Rincon, F., Strasser, S., y Toqué, N.: 2007a, Astron. Astrophys 475, 1053

Aurière, M., Wade, G. A., Silvester, J., Lignieres, F., Bagnulo, S., Bale, K., Dintrans, B., Donati, J. F., Folsom, C. P., Gruberbauer, M., Hui Bon Hoa, A., Jeffers, S., Johnson, N., Landstreet, J. D., Lebre, A., Lueftinger, T., Marsden, S., Mouillet, D., Naseri, S., Paletou, F., Petit, P., Power, J., Rincon, F., Strasser, S., y Toque, N.: 2007b, VizieR Online Data Catalog 347 
Babcock, H. W.: 1947, Astrophys. J. 105, 105

Babcock, H. W.: 1960a, in J. L. Greenstein (ed.), Stellar Atmospheres, p. 282

Babcock, H. W.: 1960b, Astrophys. J. 132, 521

Bagnulo, S., Landi Degl'Innocenti, M., Landolfi, M., y Mathys, G.: 2002, Astron. Astrophys 394, 1023

Bernacca, P. L.: 1968, in Società Astronomica Italiana Meeting XI, Vol. 11, p. 171

Bidelman, W. P.: 1962a, Astrophys. J. 135, 651

Bidelman, W. P.: 1962b, AJ 67, 111

Bidelman, W. P.: 1965, AJ 70, 667

Bidelman, W. P. y Corliss, C. H.: 1962, Astrophys. J. 135, 968

Bohlender, D. A.: 1994, in L. A. Balona, H. F. Henrichs, y J. M. Le Contel (eds.), Pulsation; Rotation; and Mass Loss in Early-Type Stars, Vol. 162 of IAU Symposium, pp 155-166

Bohlender, D. A., Landstreet, J. D., Brown, D., y Thompson, I. B.: 1987, Astrophys. J. 323, 325

Böhm-Vitense, E.: 1967, in W. R. Hindmarsh y F. J. Lowes (eds.), Magnetism and the Cosmos, p. 179

Bond, H. E. y Levato, H.: 1976, PASP 88, 905

Borra, E. F.: 1980, Astrophys. J. 235, 915

Borra, E. F. y Landstreet, J. D.: 1979, Astrophys. J. 228, 809

Borra, E. F. y Landstreet, J. D.: 1980, Astrophys. J. Suppl. Ser. 42, 421

Borra, E. F., Landstreet, J. D., y Mestel, L.: 1982, Annu. Rev. Astron. Astrophys. 20, 191

Borra, E. F., Landstreet, J. D., y Thompson, I.: 1983, Astrophys. J. Suppl. Ser. 53, 151

Borra, E. F. y Vaughan, A. H.: 1976, Astrophys. J. Lett. 210, L145

Bouret, J.-C., Donati, J.-F., Martins, F., Escolano, C., Marcolino, W., Lanz, T., y Howarth, I. D.: 2008, Mon. Not. R. Astron. Soc. 389, 75

Braithwaite, J.: 2006a, Astron. Astrophys 449, 451

Braithwaite, J.: 2006b, Astron. Astrophys 453, 687 
Braithwaite, J.: 2007, Astron. Astrophys 469, 275

Braithwaite, J. y Nordlund, Å.: 2006, Astron. Astrophys 450, 1077

Braithwaite, J. y Spruit, H. C.: 2004, Nature 431, 819

Browning, M. K.: 2008, Astrophys. J. 676, 1262

Brun, A. S., Browning, M. K., y Toomre, J.: 2005, Astrophys. J. 629, 461

Burgers, J. M.: 1969, Flow Equations for Composite Gases, Academic Press

Cannon, A. J. y Pickering, E. C.: 1901, Annals of Harvard College Observatory 28, 129

Cannon, A. J. y Pickering, E. C.: 1912a, Annals of Harvard College Observatory 56, 65

Cannon, A. J. y Pickering, E. C.: 1912b, Annals of Harvard College Observatory 56, 115

Carpenter, K. G.: 1985, Astrophys. J. 289, 660

Catalano, F. A. y Leone, F.: 1996, Astron. Astrophys 311, 230

Chapman, S. y Cowling, T. G.: 1970, The mathematical theory of non-uniform gases. an account of the kinetic theory of viscosity, thermal conduction and diffusion in gases, Cambridge: University Press, 1970, 3rd ed.

Charbonneau, P. y MacGregor, K. B.: 2001, Astrophys. J. 559, 1094

Cowling, T. G.: 1945, Mon. Not. R. Astron. Soc. 105, 166

Cowling, T. G. y Lindsay, R. B.: 1957, Physics Today 10, 40

Deridder, G. y van Renspergen, W.: 1976, Astron. Astrophys. Suppl. 23, 147

Deutsch, A. J.: 1958, in B. Lehnert (ed.), Electromagnetic Phenomena in Cosmical Physics, Vol. 6 of IAU Symposium, p. 209

Dimitrijevic, M. S. y Sahal-Brechot, S.: 1990, Astron. Astrophys. Suppl. 82, 519

Dobler, W., Stix, M., y Brandenburg, A.: 2006, Astrophys. J. 638, 336

Dolk, L., Wahlgren, G. M., y Hubrig, S.: 2003, Astron. Astrophys 402, 299

Donati, J.-F.: 2001, in H. M. J. Boffin, D. Steeghs, y J. Cuypers (eds.), Astrotomography, Indirect Imaging Methods in Observational Astronomy, Vol. 573 of Lecture Notes in Physics, Berlin Springer Verlag, p. 207

Donati, J.-F.: 2006, in 36th COSPAR Scientific Assembly, Vol. 36 of COSPAR Meeting 
Donati, J.-F. y Landstreet, J. D.: 2009, Annu. Rev. Astron. Astrophys. 47, 333

Dworetsky, M. M.: 1973, Astrophys. J. Lett. 184, L75

Feautrier, P.: 1964, SAO Special Report 167, 80

Floquet, M.: 1979, Astron. Astrophys 77, 263

Fossati, L., Castro, N., Schöller, M., Hubrig, S., Langer, N., Morel, T., Briquet, M., Herrero, A., Przybilla, N., Sana, H., Schneider, F. R.Ñ., de Koter, A., y BOB Collaboration: 2015, Astron. Astrophys 582, A45

Fossati, L., Schneider, F. R.Ñ., Castro, N., Langer, N., Simón-Díaz, S., Müller, A., de Koter, A., Morel, T., Petit, V., Sana, H., y Wade, G. A.: 2016, Astron. Astrophys 592, A84

Garrison, R. F.: 1973, in C. Fehrenbach y B. E. Westerlund (eds.), Spectral Classification and Multicolour Photometry, Vol. 50 of IAU Symposium, p. 13

Garrison, R. F. y Gray, R. O.: 1994, AJ 107, 1556

Gieske, H. A. y Griem, H. R.: 1969, Astrophys. J. 157, 963

Gold, T.: 1968, Nature 218, 731

Goncharskii, A. V., Ryabchikova, T. A., Stepanov, V. V., Khokhlova, V. L., y Yagola, A. G.: 1983, Soviet Astronomy 27, 49

Gray, R. O.: 1997, in A. G. D. Philip, J. Liebert, R. Saffer, y D. S. Hayes (eds.), The Third Conference on Faint Blue Stars, p. 237

Griem, H. R.: 1960, Astrophys. J. 132, 883

Griem, H. R.: 1968, Astrophys. J. 154, 1111

Griem, H. R.: 1974, Spectral line broadening by plasmas, New York, Academic Press, Inc. (Pure and Applied Physics. Volume 39), 1974. 421 p.

Grunhut, J. H., Wade, G. A., y MiMeS Collaboration: 2012, in L. Drissen, C. Robert, N. St-Louis, y A. F. J. Moffat (eds.), Proceedings of a Scientific Meeting in Honor of Anthony F. J. Moffat, Vol. 465 of Astronomical Society of the Pacific Conference Series, p. 42

Gustafsson, B.: 1971, Astron. Astrophys 10, 187

Gustafsson, B. y Nissen, P. E.: 1972, Astron. Astrophys 19, 261

Hale, G. E.: 1908, Astrophys. J. 28, 315 
Hartoog, M. R. y Cowley, A. P.: 1979, Astrophys. J. 228, 229

Havnes, O. y Conti, P. S.: 1971, Astron. Astrophys 14, 1

Henrichs, H. F., de Jong, J. A., Verdugo, E., Schnerr, R. S., Neiner, C., Donati, J.-F., Catala, C., Shorlin, S. L. S., Wade, G. A., Veen, P. M., Nichols, J. S., Damen, E. M. F., Talavera, A., Hill, G. M., Kaper, L., Tijani, A. M., Geers, V. C., Wiersema, K., Plaggenborg, B., y Rygl, K. L. J.: 2013, Astron. Astrophys 555, A46

Hummer, D. G. y Mihalas, D.: 1988, Astrophys. J. 331, 794

Hunger, K. y Groote, D.: 1999, Astron. Astrophys 351, 554

Iben, Jr., I. y MacDonald, J.: 1985, Astrophys. J. 296, 540

Jaschek, C. y Jaschek, M.: 1987, Sky Telesc. 74, 612

Jaschek, M. y Jaschek, C.: 1958, Zeitschrift für Astrophysik 45, 35

Jaschek, M., Jaschek, C., y Arnal, M.: 1969, PASP 81, 650

Kemp, J. C., Swedlund, J. B., Landstreet, J. D., y Angel, J. R. P.: 1970, Astrophys. J. Lett. 161, L77

Khokhlova, V. L. y Pavlova, V. M.: 1984, Soviet Astronomy Letters 10, 158

Khokhlova, V. L., Rice, J. B., y Wehlau, W. H.: 1986, Astrophys. J. 307, 768

Khokhlova, V. L., Želwanowa, E. I., Schöneich, W., y Bolton, C. T.: 1991, in D. Baade (ed.), European Southern Observatory Conference and Workshop Proceedings, Vol. 36 of European Southern Observatory Conference and Workshop Proceedings, p. 161

Khokhlova, V. L., Vasilchenko, D. V., Stepanov, V. V., y Romanyuk, I. I.: 2000, Astronomy Letters 26, 177

Khokhlova, V. L., Vasilchenko, D. V., Stepanov, V. V., y Tsymbal, V. V.: 1997, Astronomy Letters 23, 465

Khokhlova, V. L., Vasilchenko, D. V., Stepanov, V. V., y Tsymbal, V. V.: 1998, Contributions of the Astronomical Observatory Skalnate Pleso 27, 427

Kochukhov, O.: 2014, in G. Mathys, E. R. Griffin, O. Kochukhov, R. Monier, y G. M. Wahlgren (eds.), Putting A Stars into Context: Evolution, Environment, and Related Stars, pp 389-397

Kochukhov, O. y Bagnulo, S.: 2006, Astron. Astrophys 450, 763 
Kochukhov, O., Bagnulo, S., Wade, G. A., Sangalli, L., Piskunov, N., Landstreet, J. D., Petit, P., y Sigut, T. A. A.: 2004, Astron. Astrophys 414, 613

Kodaira, K. y Unno, W.: 1969, Astrophys. J. 157, 769

Krause, F. y Oetken, L.: 1976, in W. W. Weiss, H. Jenkner, y H. J. Wood (eds.), IAU Colloq. 32: Physics of Ap Stars, p. 29

Krause, F. y Raedler, K.-H.: 1980, Mean-field magnetohydrodynamics and dynamo theory, Oxford, Pergamon Press, Ltd.

Krtička, J., Janík, J., Marková, H., Mikulášek, Z., Zverko, J., Prvák, M., y Skarka, M.: 2013, Astron. Astrophys 556, A18

Kurtz, D. W.: 1978, Information Bulletin on Variable Stars 1436

Lamers, H. J. G. L. M. y Cassinelli, J. P.: 1999, Introduction to Stellar Winds, Cambridge, UK: Cambridge University Press

Landstreet, J. D.: 1970, Astrophys. J. 159, 1001

Landstreet, J. D.: 1987, Mon. Not. R. Astron. Soc. 225, 437

Landstreet, J. D. y Borra, E. F.: 1978, Astrophys. J. Lett. 224, L5

LeBlanc, F., Michaud, G., y Babel, J.: 1994, Astrophys. J. 431, 388

Ledoux, P. y Renson, P.: 1966, Annu. Rev. Astron. Astrophys. 4, 293

Lehmann, H., Tkachenko, A., Fraga, L., Tsymbal, V., y Mkrtichian, D. E.: 2007, Astron. Astrophys 471, 941

Levato, H. y Malaroda, S.: 1979, PASP 91, 789

Lueftinger, T.: 2014, in G. Mathys, E. R. Griffin, O. Kochukhov, R. Monier, y G. M. Wahlgren (eds.), Putting A Stars into Context: Evolution, Environment, and Related Stars, pp 157-165

Lyubimkov, L. S.: 1984, Astrophysics 20, 255

Lyubimkov, L. S.: 1988, Astrophysics 29, 704

Lyubimkov, L. S.: 1989, Astrophysics 30, 58

Lyubimkov, L. S.: 1991, in G. Michaud y A. V. Tutukov (eds.), Evolution of Stars: the Photospheric Abundance Connection, Vol. 145 of IAU Symposium, p. 125 
MacDonald, J. y Mullan, D. J.: 2004, Mon. Not. R. Astron. Soc. 348, 702

MacGregor, K. B. y Cassinelli, J. P.: 2003, Astrophys. J. 586, 480

Madej, J.: 1983, Acta Astron. 33, 1

Maeder, A.: 1987, Astron. Astrophys 173, 247

Maeder, A. y Meynet, G.: 2003, Astron. Astrophys 411, 543

Maeder, A. y Meynet, G.: 2004, Astron. Astrophys 422, 225

Maeder, A. y Meynet, G.: 2005, Astron. Astrophys 440, 1041

Mathys, G. y Hubrig, S.: 1995, Astron. Astrophys 293, 810

Maury, A. C. y Pickering, E. C.: 1897, Annals of Harvard College Observatory 28, 1

Mestel, L.: 1999, Stellar magnetism, Oxford : Clarendon, 1999. (International series of monographs on physics ; 99)

Michaud, G.: 1970, Astrophys. J. 160, 641

Michaud, G.: 1980, AJ 85, 589

Moffatt, H. K.: 1978, Magnetic field generation in electrically conducting fluids, Cambridge, England, Cambridge University Press, 1978. 353 p.

Morgan, W. W.: 1931, Astrophys. J. 73, 104

Morgan, W. W., Keenan, P. C., y Kellman, E.: 1943, An atlas of stellar spectra, with an outline of spectral classification, Chicago, Ill., The University of Chicago press [1943]

Moss, D.: 2001, in G. Mathys, S. K. Solanki, y D. T. Wickramasinghe (eds.), Magnetic Fields Across the Hertzsprung-Russell Diagram, Vol. 248 of Astronomical Society of the Pacific Conference Series, p. 305

Muchmore, D. O.: 1980, Ph.D. thesis, Washington Univ., Seattle.

Mullan, D. J. y MacDonald, J.: 2005, Mon. Not. R. Astron. Soc. 356, 1139

Nayfonov, A., Däppen, W., Hummer, D. G., y Mihalas, D.: 1999, Astrophys. J. 526, 451

Neiner, C., Henrichs, H. F., Floquet, M., Frémat, Y., Preuss, O., Hubert, A.-M., Geers, V. C., Tijani, A. H., Nichols, J. S., y Jankov, S.: 2003, Astron. Astrophys 411, 565

Oetken, L.: 1977, Astronomische Nachrichten 298, 197 
Oetken, L.: 1979, Astronomische Nachrichten 300, 1

Osawa, K.: 1965, Annals of the Tokyo Astronomical Observatory 9, 121

Panei, J. A., Althaus, L. G., Chen, X., y Han, Z.: 2007, Mon. Not. R. Astron. Soc. 382, 779

Paquette, C., Pelletier, C., Fontaine, G., y Michaud, G.: 1986, Astrophys. J. Suppl. Ser. 61, 177

Parker, E.N.: 1979, Cosmical magnetic fields: Their origin and their activity, Oxford, Clarendon Press; New York, Oxford University Press, 1979, 858 p.

Petit, P., Dintrans, B., Aurière, M., Catala, C., Donati, J.-F., Fares, R., Gastine, T., Lignières, F., Morin, J., Paletou, F., Ramirez, J., Solanki, S. K., y Théado, S.: 2008, in C. Charbonnel, F. Combes, y R. Samadi (eds.), SF2A-2008, p. 523

Petit, V., Owocki, S. P., Wade, G. A., Cohen, D. H., Sundqvist, J. O., Gagné, M., Maíz Apellániz, J., Oksala, M. E., Bohlender, D. A., Rivinius, T., Henrichs, H. F., Alecian, E., Townsend, R. H. D., ud-Doula, A., y MiMeS Collaboration: 2013, Mon. Not. R. Astron. Soc. 429, 398

Pikelner, S. B.: 1966, Principles of cosmic electrodynamics, Moscow: Nauka

Polosukhina, N., Shulyak, D., Shavrina, A., Lyashko, D., Drake, N., Glagolevsky, Y., Kudryavtsev, D., y Smirnova, M.: 2014, in G. Mathys, E. R. Griffin, O. Kochukhov, R. Monier, y G. M. Wahlgren (eds.), Putting A Stars into Context: Evolution, Environment, and Related Stars, pp 166-171

Preston, G. W.: 1969a, Astrophys. J. 157, 247

Preston, G. W.: 1969b, Astrophys. J. 156, 967

Preston, G. W.: 1969c, Astrophys. J. 158, 243

Preston, G. W.: 1970, Astrophys. J. 160, 1059

Preston, G. W.: 1971, Astrophys. J. 164, 309

Preston, G. W.: 1974, Annu. Rev. Astron. Astrophys. 12, 257

Pyper, D. M.: 1969, Astrophys. J. Suppl. Ser. 18, 347

Richtmyer, R. D. y Morton, K. W.: 1994, Difference methods for initial-value problems, Malabar, Fla.: Krieger Publishing Co., —c1994, 2nd ed. 
Rivinius, T., Stahl, O., Baade, D., y Kaufer, A.: 2003, Information Bulletin on Variable Stars 5397

Rivinius, T., Wade, G. A., Townsend, R. H. D., Shultz, M., Grunhut, J. H., Stahl, O., y Stahl: 2011, in C. Neiner, G. Wade, G. Meynet, y G. Peters (eds.), Active OB Stars: Structure, Evolution, Mass Loss, and Critical Limits, Vol. 272 of IAU Symposium, pp $210-211$

Robinson, R. D., Worden, S. P., y Harvey, J. W.: 1980, Astrophys. J. Lett. 236, L155

Rohrmann, R. D.: 2001, Mon. Not. R. Astron. Soc. 323, 699

Rohrmann, R. D., Serenelli, A. M., Althaus, L. G., y Benvenuto, O. G.: 2002, Mon. Not. R. Astron. Soc. 335, 499

Rusomarov, N., Kochukhov, O., Ryabchikova, T., y Piskunov, N.: 2015, Astron. Astrophys 573, A123

Rybicki, G. B.: 1971, Journal of Quantitative Spectroscopy and Radiative Transfer 11, 589

Sargent, A. W. L. W. y Jugaku, J.: 1961, Astrophys. J. 134, 777

Schlüter, A.: 1950, Zeitschrift Naturforschung Teil A 5, 72

Sharpless, S.: 1952a, AJ 57, 27

Sharpless, S.: 1952b, Astrophys. J. 116, 251

Shultz, M., Rivinius, T., Folsom, C. P., Wade, G. A., Townsend, R. H. D., Sikora, J., Grunhut, J., Stahl, O., y MiMeS Collaboration: 2015, Mon. Not. R. Astron. Soc. 449, 3945

Shulyak, D., Kochukhov, O., Valyavin, G., Lee, B.-C., Galazutdinov, G., Kim, K.-M., Han, I., y Burlakova, T.: 2010, Astron. Astrophys 509, A28

Shulyak, D., Valyavin, G., Kochukhov, O., Lee, B.-C., Galazutdinov, G., Kim, K.-M., Han, I., Burlakova, T., Tsymbal, V., y Lyashko, D.: 2007, Astron. Astrophys 464, 1089

Silvester, J., Kochukhov, O., y Wade, G. A.: 2014, Mon. Not. R. Astron. Soc. 444, 1442

Smith, K. C.: 1996, Astrophysics $\&$ Space Science 237, 77

Spruit, H. C.: 2002, Astron. Astrophys 381, 923

Stȩpień, K.: 2000, Astron. Astrophys 353, 227

Stibbs, D. W.: 1950, Mon. Not. R. Astron. Soc. 110, 395 
Tayler, R. J.: 1973, Mon. Not. R. Astron. Soc. 161, 365

Thompson, I. B.: 1983, Mon. Not. R. Astron. Soc. 205, 43P

Titus, J. y Morgan, W. W.: 1940, Astrophys. J. 92, 256

Townsend, R. H. D. y Owocki, S. P.: 2005, Mon. Not. R. Astron. Soc. 357, 251

Underhill, A. B., Fahey, R. P., y Klinglesmith, D. A.: 1975, Astrophys. J. 199, 120

Vallverdú, R., Cidale, L., Rohrmann, R., y Ringuelet, A.: 2014, Astrophysics E Space Science 352, 95

Vallverdú, R., Venero, R., Cidale, L., Rorhmann, R., y Curé, M.: 2007, Boletin de la Asociacion Argentina de Astronomia La Plata Argentina 50, 165

Valyavin, G., Kochukhov, O., y Piskunov, N.: 2004, Astron. Astrophys 420, 993

Vauclair, S. y Vauclair, G.: 1982, Annu. Rev. Astron. Astrophys. 20, 37

Wade, G. A., Bohlender, D. A., Brown, D., Elkin, V. G., Landstreet, J. D., y Romanyuk, I. I.: 1997, Astron. Astrophys 320, 172

Wade, G. A., Kudryavtsev, D., Romanyuk, I. I., Landstreet, J. D., y Mathys, G.: 2000, Astron. Astrophys 355, 1080

Wade, G. A. y MiMeS Collaboration: 2015, in Y. Y. Balega, I. I. Romanyuk, y D. O. Kudryavtsev (eds.), Physics and Evolution of Magnetic and Related Stars, Vol. 494 of Astronomical Society of the Pacific Conference Series, p. 30

Wade, G. A., Neiner, C., Alecian, E., Grunhut, J. H., Petit, V., Batz, B. d., Bohlender, D. A., Cohen, D. H., Henrichs, H. F., Kochukhov, O., Landstreet, J. D., Manset, N., Martins, F., Mathis, S., Oksala, M. E., Owocki, S. P., Rivinius, T., Shultz, M. E., Sundqvist, J. O., Townsend, R. H. D., ud-Doula, A., Bouret, J.-C., Braithwaite, J., Briquet, M., Carciofi, A. C., David-Uraz, A., Folsom, C. P., Fullerton, A. W., Leroy, B., Marcolino, W. L. F., Moffat, A. F. J., Nazé, Y., Louis, N. S., Aurière, M., Bagnulo, S., Bailey, J. D., Barbá, R. H., Blazère, A., Böhm, T., Catala, C., Donati, J.-F., Ferrario, L., Harrington, D., Howarth, I. D., Ignace, R., Kaper, L., Lüftinger, T., Prinja, R., Vink, J. S., Weiss, W. W., y Yakunin, I.: 2016, Mon. Not. R. Astron. Soc. 456, 2

Wehlau, W., Rice, J., Piskunov, N. E., y Khokhlova, V. L.: 1982, Soviet Astronomy Letters 8, 30

Wehlau, W. H., Rice, J. B., y Khokhlova, V. L.: 1991, Astronomical and Astrophysical Transactions 1, 55 
Wolff, S. C. y Morrison, N. D.: 1974, PASP 86, 935

Wolff, S. C. y Wolff, R. J.: 1970, Astrophys. J. 160, 1049

Wright, G. A. E.: 1973, Mon. Not. R. Astron. Soc. 162, 339

Wrubel, M. H.: 1952, Astrophys. J. 116, 291

Yakunin, I., Wade, G., Bohlender, D., Kochukhov, O., Marcolino, W., Shultz, M., Monin, D., Grunhut, J., Sitnova, T., Tsymbal, V., y MiMeS Collaboration: 2015, Mon. Not. R. Astron. Soc. 447, 1418

Zboril, M., North, P., Glagolevskij, Y. V., y Betrix, F.: 1997, Astron. Astrophys 324, 949 


\section{Apéndice A}

\section{Anexo I. Los códigos}

\section{A.1 Modelo de atmósfera sin campo magnético}

Para calcular la estructura de la atmósfera de una estrella tipo B utilizamos el código desarrollado por Rohrmann 2001) y que fuera mejorado posteriormente por Rohrmann et al. 2002 .

El programa, desarrollado en lenguaje FORTRAN 7 \%, resuelve una atmósfera compuesta principalmente por $\mathrm{H}$ y He.

En este código numérico, se modela una atmósfera no gris bajo las siguientes hipótesis:

- Geometría plana y paralela.

- Gravedad constante.

- Equilibrio termodinámico local (ETL).

- Flujo de energía constante.

- Composición química homogénea.

- Ausencia de campos magnéticos.

Este código brinda la marcha de la densidad de masa, la presión, la temperatura y las densidades de número de partículas en cada capa. La variable independiente del modelo es la profundidad óptica $\tau$ correspondiente a la opacidad media de Rosseland. Las estructuras de densidad y presión son evaluadas en cada iteración numérica integrando la ecuación de equilibrio hidrostático. Se define una estructura de temperatura inicial (correspondiente al modelo gris) y ésta se corrige posteriormente considerando el balance de energía al calcular el espectro emergente. 
Los parámetros de entrada del modelo de atmósfera son: la temperatura efectiva de la estrella (en grados Kelvin), el radio (en metros), la gravedad (en logaritmo decimal) y la abundancia fraccional de masa de He en la superficie de la estrella que se desea modelar. Estos parámetros definen unívocamente al modelo de atmósfera de la estrella.

Además de resolver la estructura de la atmósfera, el programa contiene subrutinas que permiten calcular el espectro de líneas emergentes de las diferentes especies químicas, en nuestro caso, las líneas de H y He.

Las ecuaciones de transporte radiativo y de flujo de energía se resuelven por medio de una técnica de linealización estándar. Específicamente, la ecuación de transporte radiativo (formulada en términos de los factores de Eddington) y la condición de flujo constante, se resuelven mediante un proceso iterativo sobre ecuaciones linealizadas para un grillado de puntos de profundidad óptica y un grillado de frecuencias siguiendo el método de Feautrier. (ver Feautrier 1964).

La corrección de temperatura en cada capa se obtiene de la condición de equilibrio radiativo utilizando el procedimiento de Rybicki (1971), en donde los sistemas de ecuaciones lineales resultantes no están agrupadas en bloques de profundidad óptica, sino en bloques de frecuencias (ver Gustafsson, 1971, Gustafsson y Nissen, 1972.

La configuración atómica adoptada consta de 100 niveles ligados para el H I y el He II, y 144 niveles ligados para modelar el átomo de He I. Las poblaciones atómicas se calculan dentro del formalismo de probabilidad ocupacional de Hummer y Mihalas 1988 para el tratamiento de los efectos no ideales en la ecuación de estado del gas. Este formalismo evita los truncamientos típicos de las funciones de partición de las partículas y da una manera termodinámicamente auto-consistente para evaluar el equilibrio de ionización y la excitación en el gas. Específicamente, las densidades de números de partículas de las poblaciones atómicas de una especie dada en el estado de energía $i\left(n_{i}\right)$, en relación con la densidad total de número de partículas de cada especie, $n_{t o t}$, está dada por:

$$
\frac{n_{i}}{n_{\mathrm{tot}}}=\frac{w_{i} g_{i} e^{-E_{i} / k T}}{U},
$$

donde $w_{i}$ es la probabilidad de ocupación del nivel de $i, g_{i}$ es el peso estadístico del nivel, $E_{i}$ es la energía de excitación respecto del nivel fundamental, $U$ es la función de partición interna, $k$ es la constante de Boltzmann y $T$ la temperatura del gas. Los factores $w_{i}$ se suman sobre las perturbaciones de los niveles atómicos debidas a partículas neutras y cargadas teniendo en cuenta el tamaño finito de las especies con electrones ligados y los efectos del campo eléctrico resultante producido por los iones y electrones. En particular, los efectos de carga se basan en un ajuste cuántico de la teoría de ionización Stark Nayfonov et al. 1999. 
Es necesario dar explícitamente las líneas que se desean calcular. Se debe indicar los parámetros de las líneas (elemento químico, número atómico, estado de ionización, niveles involucrados en la transición, los pesos estadísticos de cada nivel, la energía de la transición, la intensidad del oscilador, la longitud de onda de la línea) y resolución espectral (cantidad de puntos de frecuencia -o longitud de onda- con los cuales se muestra a la línea).

El programa proveé varios archivos de salida (en principio 10) con distintos parámetros. En particular a nosotros nos interesan los siguientes:

- La estructura de la atmósfera (w_atm.dat). Este archivo nos da para cada profundidad óptica $\tau$, el logaritmo de la temperatura, el logaritmo de la presión del gas, el logaritmo de la densidad, la fracción de flujo radiativo, la fracción del flujo convectivo, el gradiente real de temperatura, el gradiente adiabático, la coordenada radial $(\mathrm{km})$, la densidad de columna de masa $\left(\mathrm{g} / \mathrm{cm}^{2}\right)$ y masa de la capa $(\mathrm{g})$ entre $\tau$ y $\tau+\Delta \tau$.

- Las densidades de números de partículas de las distintas especies químicas (w_pob.dat). Para cada capa y temperatura, tenemos: logaritmo de la densidad de núméro de electrones, logaritmo de la densidad de núméro de $\mathrm{H}$, logaritmo de la densidad de número de He, fracción de masa de protones (hidrógeno ionizado, H II), fracción de masa de hidrógeno molecular $\left(H_{2}\right)$, fracción de masa de He neutro (He I) y la fracción de masa de He dos veces ionizado (He III). A partir de las fracciones de masa podemos calcular las respectivas densidades de números de partículas en cada estado de ionización, e incluso las correspondientes al deuterio.

- El espectro de líneas resultante ( $\left.w_{-} f l i . d a t\right)$. Los datos suministrados son: longitud de onda $(\AA)$, frecuencia $(H z)$, energía $(e V)$, flujo de Eddington emergente por unidad de longitud de onda, flujo de Planck por unidad de longitud de onda, flujo de Eddington emergente por unidad de frecuencia, flujo de Planck por unidad de frecuencia.

- Datos fotométricos (w_col.dat). Nos brinda: temperatura efectiva, los índices de color U-B, B-V, V-R, V-K, R-I, J-H, H-K y K-L, la corrección bolométrica y la magnitud visual absoluta.

\section{A.2 Gravedad efectiva}

Este programa se desarrolló para poder incluir los efectos del campo magnético, siguiendo las ecuaciones publicadas por Valyavin et al. 2004), explicadas en el capítulo \$3. El código fue programado en FORTRAN rry pero también realizamos una versión en FORTRAN 95. El programa tiene un archivo de entrada en donde se ingresan la intensidad del campo eléctrico (en unidades del sistema CGS), la intensidad del campo magnético (en Gauss), el 
radio de la estrella (en kilómetros) y la latitud magnética (en unidades de $\pi$ radianes). Por otro lado, lee del programa que calcula la estructura de la atmósfera, los archivos w_atm.dat (estructura de la atmósfera) y w_pob.dat (densidad de número de partículas de las poblaciones). Del primero lee la profundidad óptica, la temperatura, la densidad y profundidad en kilómetros de cada capa. Del segundo lee las densidades de número de partículas de cada una de las poblaciones de cada especie química a cada profundidad.

Como salida, el programa devuelve un archivo conteniendo el logaritmo de la profundidad óptica, la gravedad efectiva, el logaritmo de la gravedad efectiva, y el cociente de la gravedad efectiva respecto a la gravedad superficial inicial, para cada una de las capas del modelo.

También realizamos una versión del programa que nos permite calcular la estructura de la atmósfera considerando un campo magnético integrado sobre todo el disco de la estrella, para una dada fase de rotación según se detalla en la sección 6.1 .

Esta versión utiliza también los archivos w_atm.dat y w_pob.dat y nos devuelve una salida de datos igual que la correspondiente a modelos locales. La diferencia radica en el archivo de entrada, en donde se dan como parámetros iniciales, $\langle B\rangle$, la intensidad integrada del campo magnético, y $\left\langle B_{\theta} \operatorname{sen} \theta>\right.$, el valor integrado de la componente latitudinal del campo magnético multiplicada por el seno de la latitud, en vez de la intensidad del campo magnético.

\section{A.3 Acoplamiento de la gravedad efectiva con la es- tructura de la atmósfera}

Para obtener los resultados finales debemos acoplar al programa que calcula la estructura de la atmósfera presentado en la sección A.1, y el programa que calcula la gravedad efectiva (sección $\$$ A.2). A tal fin realizamos un procedimiento iterativo.

Para ello primero calculamos la estructura de una atmósfera tomando una gravedad superficial constante con la profundidad. Una vez obtenido el modelo de atmósfera $(\mathrm{P}, \rho, \mathrm{T})$, con él calculamos la gravedad efectiva para cada capa.

A continuación, utilizamos el programa que resuelve la atmósfera, pero con la modificación de que en vez de usar un valor constante para la gravedad superficial en cada capa, usa los valores correspondientes obtenidos con el programa de la gravedad efectiva, para calcular la nueva estructura de la atmósfera. Con este nuevo modelo calculamos nuevamente una gravedad efectiva para cada capa. Como es de esperar, estos valores de la gravedad efectiva no coinciden con los calculados previamente. Debemos entonces resolver nuevamente la atmósfera repitiendo el procedimiento descripto hasta que los valores obtenidos para la gravedad efectiva difieran con los calculados en el paso anterior en un porcentaje chico, 
por ejemplo, menor a $0.1 \%$ en todas las capas. Diremos que el modelo ha convergido y consideraremos los resultados obtenidos en la última iteración.

Con repetir el procedimiento iterativo entre cinco y siete veces es suficiente para alcanzar la convergencia.

\section{A.4 Normalización de las líneas espectrales y anchos equivalentes.}

Para poder comparar las líneas de nuestros distintos modelos, debemos normalizarlas. Con tal fin realizamos un programa en FORTRAN 77 que lee el archivo w_fli.dat, y fijando los valores en donde las alas de las líneas se unen con el continuo, nos devuelve el perfil normalizado de la línea en un archivo y el ancho equivalente de la misma.

Para calcular el perfil normalizado, el programa aproxima el continuo mediante interpolación lineal entre los dos puntos seleccionados que constituyen los bordes de la línea. Luego, para cada punto (longitud de onda) se divide el valor del flujo por el valor interpolado del continuo.

Una vez obtenido el perfil normalizado, mediante una integración por el método de trapecios, se obtiene el valor del ancho equivalente. El código además determina el valor de la intensidad en el centro de la línea.

También hemos realizado una versión de este programa en FORTRAN 95, más general, en donde todos los parámetros necesarios se ingresan mediante un archivo de entrada.

\section{A.5 Difusión}

Para implementar los procesos de difusión, desarrollé en colaboración con el Dr. Jorge Panei, un programa en FORTRAN 7\%, escrito en forma modular para permitir su posterior pasaje a FORTRAN 95. El mismo está basado en el formalismo dado por Iben y MacDonald 1985, y calcula los coeficientes resistivos necesarios empleando las fórmulas y tablas suministradas en el trabajo de Paquette et al. 1986.

El programa requiere las fracciones de masa de cada especie química en cada capa de la atmósfera, así como la temperatura y el valor de la gravedad efectiva. Para ello generamos el archivo con los datos de entrada necesarios a partir de los archivos w_atm.dat y w_pob.dat y del archivo que contiene los valores de la gravedad efectiva. Este modelo inicial a tiempo $t=0$ es el que vamos a difundir.

En otro archivo, indicamos el tiempo durante el cual estará actuando la difusión y en cuantos intervalos dividimos ese tiempo para realizar los cálculos. Aquí también se indica el nombre 
del archivo en donde se encuentra los datos del modelo a difundir. Luego se indican la cantidad total de isótopos presentes en el modelo y la cantidad de isótopos que se van a difundir. Los siguientes valores indican cuántas columnas contiene el archivo con el modelo y a qué número de columna corresponde el logaritmo de la profundidad óptica (que caracteriza a cada capa), el logaritmo de la temperatura, la densidad másica, la densidad numérica de electrones, la coordenada radial, y la gravedad efectiva. Una variable lógica indica si se escriben o no los modelos intermedios y si es afirmativo, cada cuantos modelos escribe. Finalmente, para cada isótopo que se va a difundir, se indica su número atómico, su número másico, su masa en uma y en gramos, cuantos electrones aporta a la mezcla, la carga eléctrica de cada ión y el número de columna que le corresponde.

Como salida, el programa aporta 2 archivos conteniendo las nuevas abundancias de cada especie química en cada capa después de que tuvo lugar la difusión. Uno nos da la fracción de masa y el otro la densidad numérica de cada especie. Además si se usó la opción de escribir los modelos intermedios, el programa suministrará estos dos archivos identificando en su nombre a qué intervalo de tiempo corresponde.

Con las modificaciones necesarias se ejecuta el programa que calcula la estructura de la atmósfera, leyendo la nueva distribución de abundancias, de tal forma de poder calcular el espectro después de que los elementos se hayan difundido. 


\section{Apéndice B}

\section{Anexo II. Coeficientes de Splines}

En el presente anexo, reproducimos las tablas de donde obtenemos los coeficientes de Splines, necesarios para el cálculo de la difusión. Estas tablas pertenecen al trabajo de Paquette et al. 1986.

Las tablas de las figuras B.1 a B.4 corresponden al caso en que el potencial es repulsivo, mientras que de la tabla B.5 a la B.8 corresponden al caso de un potencial atractivo. Cada grupo de tablas, corresponden a valores de $j$ entre 1 y 4 , uno por cada tabla respectivamente. 
TABLE 1

Spline Coefficients (Repulsive Potential): $j=1$

\begin{tabular}{|c|c|c|c|c|}
\hline$n$ & $c{ }_{1 n}^{(1)}$ & $c_{2 n}^{(1)}$ & $c_{3 n}^{(1)}$ & $c_{4 n}^{(1)}$ \\
\hline $\begin{array}{r}1 \\
2 \\
3 \\
4 \\
5 \\
6 \\
7 \\
8 \\
9 \\
10 \\
11 \\
12 \\
13 \\
14 \\
15 \\
16 \\
17 \\
18 \\
19 \\
20 \\
21 \\
22 \\
23 \\
24 \\
25 \\
26 \\
27 \\
28 \\
29 \\
30 \\
31 \\
32 \\
33 \\
34 \\
35 \\
36 \\
37 \\
38 \\
39 \\
40 \\
41 \\
42 \\
43 \\
44 \\
45 \\
46 \\
47 \\
48 \\
49 \\
50 \\
\end{array}$ & $\begin{array}{r}1.19599 E-02 \\
-2.39198 E-02 \\
-1.48010 E-02 \\
-1.77390 E-02 \\
-1.74423 E-02 \\
-1.80040 E-02 \\
-1.83218 E-02 \\
-1.86847 E-02 \\
-1.90073 E-02 \\
-1.93026 E-02 \\
-1.95555 E-02 \\
-1.97557 E-02 \\
-1.98886 E-02 \\
-1.99373 E-02 \\
-1.98810 E-02 \\
-1.96948 E-02 \\
-1.93486 E-02 \\
-1.88059 E-02 \\
-1.80227 E-02 \\
-1.69459 E-02 \\
-1.55109 E-02 \\
-1.36394 E-02 \\
-1.12361 E-02 \\
-8.18466 E-03 \\
-4.34258 E-03 \\
4.65253 E-04 \\
6.45493 E-03 \\
1.38941 E-02 \\
2.31151 E-02 \\
3.45317 E-02 \\
4.86585 E-02 \\
6.61321 E-02 \\
8.77309 E-02 \\
1.14383 E-01 \\
1.47142 E-01 \\
1.87092 E-01 \\
2.35096 E-01 \\
2.91268 E-01 \\
3.53977 E-01 \\
4.18217 E-01 \\
4.73499 E-01 \\
5.02343 E-01 \\
4.82140 E-01 \\
3.92303 E-01 \\
2.20401 E-01 \\
-5.31156 E-02 \\
-3.94063 E-01 \\
-5.99574 E-01 \\
-4.71033 E-01 \\
-4.68969 E-01\end{array}$ & $\begin{array}{l}-2.39198 E-02 \\
-1.48010 E-02 \\
-1.77390 E-02 \\
-1.74423 E-02 \\
-1.80040 E-02 \\
-1.83218 E-02 \\
-1.86847 E-02 \\
-1.90073 E-02 \\
-1.93026 E-02 \\
-1.95555 E-02 \\
-1.97557 E-02 \\
-1.98886 E-02 \\
-1.99373 E-02 \\
-1.98810 E-02 \\
-1.96948 E-02 \\
-1.93486 E-02 \\
-1.88059 E-02 \\
-1.80227 E-02 \\
-1.69459 E-02 \\
-1.55109 E-02 \\
-1.36394 E-02 \\
-1.12361 E-02 \\
-8.18466 E-03 \\
-4.34258 E-03 \\
4.65253 E-04 \\
6.45493 E-03 \\
1.38941 E-02 \\
2.31151 E-02 \\
3.45317 E-02 \\
4.86585 E-02 \\
6.61321 E-02 \\
8.77309 E-02 \\
1.14383 E-01 \\
1.47142 E-01 \\
1.87092 E-01 \\
2.35096 E-01 \\
2.91268 E-01 \\
3.53977 E-01 \\
4.18217 E-01 \\
4.73499 E-01 \\
5.02343 E-01 \\
4.82140 E-01 \\
3.92303 E-01 \\
2.20401 E-01 \\
-5.31156 E-02 \\
-3.94063 E-01 \\
-5.99574 E-01 \\
-4.71033 E-01 \\
-4.68969 E-01 \\
2.34484 E-01\end{array}$ & $\begin{array}{l}-3.02547 E+01 \\
-2.94860 E+01 \\
-2.87231 E+01 \\
-2.79637 E+01 \\
-2.72086 E+01 \\
-2.64576 E+01 \\
-2.57110 E+01 \\
-2.49688 E+01 \\
-2.42310 E+01 \\
-2.34978 E+01 \\
-2.27693 E+01 \\
-2.20454 E+01 \\
-2.13263 E+01 \\
-2.06120 E+01 \\
-1.99024 E+01 \\
-1.91976 E+01 \\
-1.84975 E+01 \\
-1.78021 E+01 \\
-1.71112 E+01 \\
-1.64246 E+01 \\
-1.57421 E+01 \\
-1.50633 E+01 \\
-1.43878 E+01 \\
-1.37150 E+01 \\
-1.30441 E+01 \\
-1.23743 E+01 \\
-1.17044 E+01 \\
-1.10329 E+01 \\
-1.03581 E+01 \\
-9.67777 E+00 \\
-8.98913 E+00 \\
-8.28881 E+00 \\
-7.57261 E+00 \\
-6.83537 E+00 \\
-6.07066 E+00 \\
-5.27065 E+00 \\
-4.42573 E+00 \\
-3.52439 E+00 \\
-2.55315 E+00 \\
-1.49695 E+00 \\
-3.40379 E-01 \\
9.29832 E-01 \\
2.32060 E+00 \\
3.82709 E+00 \\
5.42773 E+00 \\
7.08127 E+00 \\
1.02683 E+01 \\
1.16706 E+01 \\
1.29598 E+01\end{array}$ & $\begin{array}{l}-2.94860 E+01 \\
-2.87231 E+01 \\
-2.79637 E+01 \\
-2.72086 E+01 \\
-2.64576 E+01 \\
-2.57110 E+01 \\
-2.49688 E+01 \\
-2.42310 E+01 \\
-2.34978 E+01 \\
-2.27693 E+01 \\
-2.20454 E+01 \\
-2.13263 E+01 \\
-2.06120 E+01 \\
-1.99024 E+01 \\
-1.91976 E+01 \\
-1.84975 E+01 \\
-1.78021 E+01 \\
-1.71112 E+01 \\
-1.64246 E+01 \\
-1.57421 E+01 \\
-1.50633 E+01 \\
-1.43878 E+01 \\
-1.37150 E+01 \\
-1.30441 E+01 \\
-1.23743 E+01 \\
-1.17044 E+01 \\
-1.10329 E+01 \\
-1.03581 E+01 \\
-9.67777 E+00 \\
-8.98913 E+00 \\
-8.28881 E+00 \\
-7.57261 E+00 \\
-6.83537 E+00 \\
-6.07066 E+00 \\
-5.27065 E+00 \\
-4.42573 E+00 \\
-3.52439 E+00 \\
-2.55315 E+00 \\
-1.49695 E+00 \\
-3.40379 E-01 \\
9.29832 E-01 \\
2.32060 E+00 \\
3.82709 E+00 \\
5.42773 E+00 \\
7.08127 E+00 \\
8.72205 E+00 \\
1.02683 E+01 \\
1.16706 E+01 \\
1.29598 E+01 \\
1.41366 E+01\end{array}$ \\
\hline
\end{tabular}

Figura B.1: Tabla 1 del trabajo de Paquette et al. 1986. Coeficientes de Spline para un potencial repulsivo: $j=1$. 
TABLE 2

SPline Coefficients (RePulsive Potential): $j=2$

\begin{tabular}{|c|c|c|c|c|}
\hline$n$ & $c_{1 n}^{(2)}$ & $c_{2 n}^{(2)}$ & $c_{3 n}^{(2)}$ & $c_{4 n}^{(2)}$ \\
\hline $\begin{array}{r}1 \\
2 \\
3 \\
4 \\
5 \\
6 \\
7 \\
8 \\
9 \\
10 \\
11 \\
12 \\
13 \\
14 \\
15 \\
16 \\
17 \\
18 \\
19 \\
20 \\
21 \\
22 \\
23 \\
24 \\
25 \\
26 \\
27 \\
28 \\
29 \\
30 \\
31 \\
32 \\
33 \\
34 \\
35 \\
36 \\
37 \\
38 \\
39 \\
40 \\
41 \\
42 \\
43 \\
44 \\
45 \\
46 \\
47 \\
48 \\
49 \\
50\end{array}$ & $\begin{array}{l}1.34102 E-02 \\
-2.68205 E-02 \\
-1.66309 E-02 \\
-1.99547 E-02 \\
-1.96575 E-02 \\
-2.03264 E-02 \\
-2.07272 E-02 \\
-2.11843 E-02 \\
-2.16034 E-02 \\
-2.20005 E-02 \\
-2.23602 E-02 \\
-2.26732 E-02 \\
-2.29256 E-02 \\
-2.31017 E-02 \\
-2.31822 E-02 \\
-2.31439 E-02 \\
-2.29591 E-02 \\
-2.25945 E-02 \\
-2.20100 E-02 \\
-2.11574 E-02 \\
-1.99787 E-02 \\
-1.84040 E-02 \\
-1.63488 E-02 \\
-1.37108 E-02 \\
-1.03658 E-02 \\
-6.16232 E-03 \\
-9.15489 E-04 \\
5.60137 E-03 \\
1.36664 E-02 \\
2.36209 E-02 \\
3.58820 E-02 \\
5.09546 E-02 \\
6.94376 E-02 \\
9.20141 E-02 \\
1.19403 E-01 \\
1.52233 E-01 \\
1.90763 E-01 \\
2.34333 E-01 \\
2.80392 E-01 \\
3.23003 E-01 \\
3.51091 E-01 \\
3.47669 E-01 \\
2.93155 E-01 \\
1.77064 E-01 \\
1.68817 E-02 \\
-1.37836 E-01 \\
-2.36519 E-01 \\
-2.49035 E-01 \\
-1.95933 E-01 \\
-1.60453 E-01\end{array}$ & $\begin{array}{l}-2.68205 E-02 \\
-1.66309 E-02 \\
-1.99547 E-02 \\
-1.96575 E-02 \\
-2.03264 E-02 \\
-2.07272 E-02 \\
-2.11843 E-02 \\
-2.16034 E-02 \\
-2.20005 E-02 \\
-2.23602 E-02 \\
-2.26732 E-02 \\
-2.29256 E-02 \\
-2.31017 E-02 \\
-2.31822 E-02 \\
-2.31439 E-02 \\
-2.29591 E-02 \\
-2.25945 E-02 \\
-2.20100 E-02 \\
-2.11574 E-02 \\
-1.99787 E-02 \\
-1.84040 E-02 \\
-1.63488 E-02 \\
-1.37108 E-02 \\
-1.03658 E-02 \\
-6.16232 E-03 \\
-9.15489 E-04 \\
5.60137 E-03 \\
1.36664 E-02 \\
2.36209 E-02 \\
3.58820 E-02 \\
5.09546 E-02 \\
6.94376 E-02 \\
9.20141 E-02 \\
1.19403 E-01 \\
1.52233 E-01 \\
1.90763 E-01 \\
2.34333 E-01 \\
2.80392 E-01 \\
3.23003 E-01 \\
3.51091 E-01 \\
3.47669 E-01 \\
2.93155 E-01 \\
1.77064 E-01 \\
1.68817 E-02 \\
-1.37836 E-01 \\
-2.36519 E-01 \\
-2.49035 E-01 \\
-1.95933 E-01 \\
-1.60453 E-01 \\
8.02267 E-02\end{array}$ & $\begin{array}{l}-2.55941 E+01 \\
-2.48408 E+01 \\
-2.40939 E+01 \\
-2.33511 E+01 \\
-2.26130 E+01 \\
-2.18796 E+01 \\
-2.11511 E+01 \\
-2.04276 E+01 \\
-1.97091 E+01 \\
-1.89959 E+01 \\
-1.82879 E+01 \\
-1.75853 E+01 \\
-1.68881 E+01 \\
-1.61965 E+01 \\
-1.55103 E+01 \\
-1.48298 E+01 \\
-1.41548 E+01 \\
-1.34853 E+01 \\
-1.28212 E+01 \\
-1.21624 E+01 \\
-1.15087 E+01 \\
-1.08598 E+01 \\
-1.02153 E+01 \\
-9.57474 E+00 \\
-8.93745 E+00 \\
-8.30266 E+00 \\
-7.66934 E+00 \\
-7.03625 E+00 \\
-6.40181 E+00 \\
-5.76409 E+00 \\
-5.12070 E+00 \\
-4.46870 E+00 \\
-3.80447 E+00 \\
-3.12357 E+00 \\
-2.42059 E+00 \\
-1.68896 E+00 \\
-9.20788 E-01 \\
-1.06834 E-01 \\
7.63360 E-01 \\
1.70085 E+00 \\
2.71586 E+00 \\
3.81513 E+00 \\
4.99784 E+00 \\
6.25091 E+00 \\
7.54647 E+00 \\
8.84609 E+00 \\
1.01126 E+01 \\
1.13224 E+01 \\
1.24724 E+01 \\
1.35754 E+01\end{array}$ & $\begin{array}{l}-2.48408 E+01 \\
-2.40939 E+01 \\
-2.33511 E+01 \\
-2.26130 E+01 \\
-2.18796 E+01 \\
-2.11511 E+01 \\
-2.04276 E+01 \\
-1.97091 E+01 \\
-1.89959 E+01 \\
-1.82879 E+01 \\
-1.75853 E+01 \\
-1.68881 E+01 \\
-1.61965 E+01 \\
-1.55103 E+01 \\
-1.48298 E+01 \\
-1.41548 E+01 \\
-1.34853 E+01 \\
-1.28212 E+01 \\
-1.21624 E+01 \\
-1.15087 E+01 \\
-1.08598 E+01 \\
-1.02153 E+01 \\
-9.57474 E+00 \\
-8.93745 E+00 \\
-8.30266 E+00 \\
-7.66934 E+00 \\
-7.03625 E+00 \\
-6.40181 E+00 \\
-5.76409 E+00 \\
-5.12070 E+00 \\
-4.46870 E+00 \\
-3.80447 E+00 \\
-3.12357 E+00 \\
-2.42059 E+00 \\
-1.68896 E+00 \\
-9.20788 E-01 \\
-1.06834 E-01 \\
7.63360 E-01 \\
1.70085 E+00 \\
2.71586 E+00 \\
3.81513 E+00 \\
4.99784 E+00 \\
6.25091 E+00 \\
7.54647 E+00 \\
8.84609 E+00 \\
1.01126 E+01 \\
1.13224 E+01 \\
1.24724 E+01 \\
1.35754 E+01 \\
1.46398 E+01\end{array}$ \\
\hline
\end{tabular}

Figura B.2: Tabla 2 del trabajo de Paquette et al. 1986.

Coeficientes de Spline para un potencial repulsivo: $j=2$. 
TABLE 3

Spline Coefficients (Repulsive Potential): $j=3$

\begin{tabular}{|c|c|c|c|c|}
\hline$n$ & $c_{1 n}^{(3)}$ & $c_{2 n}^{(3)}$ & $c_{3 n}^{(3)}$ & $c_{4 n}^{(3)}$ \\
\hline $\begin{array}{l}1 \\
2 \\
3 \\
4 \\
5 \\
6 \\
7 \\
8 \\
9 \\
10 \\
11 \\
12 \\
13 \\
14 \\
15 \\
16 \\
17 \\
18 \\
19 \\
20 \\
21 \\
22 \\
23 \\
24 \\
25 \\
26 \\
27 \\
28 \\
29 \\
30 \\
31 \\
32 \\
33 \\
34 \\
35 \\
36 \\
37 \\
38 \\
39 \\
40 \\
41 \\
42 \\
43 \\
44 \\
45 \\
46 \\
47 \\
48 \\
49 \\
50\end{array}$ & 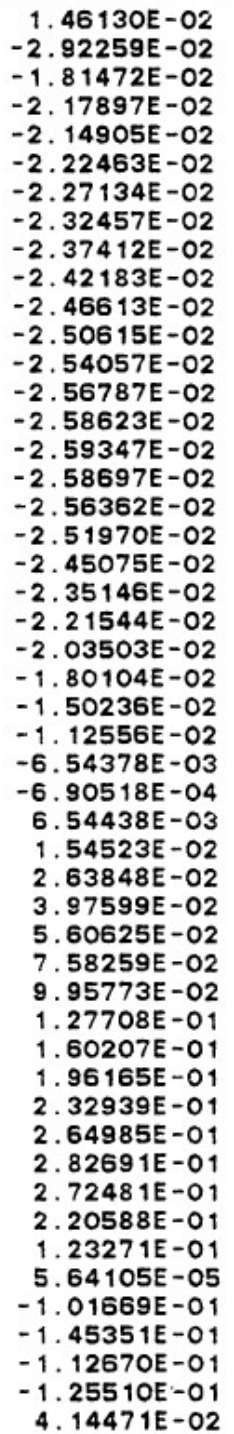 & 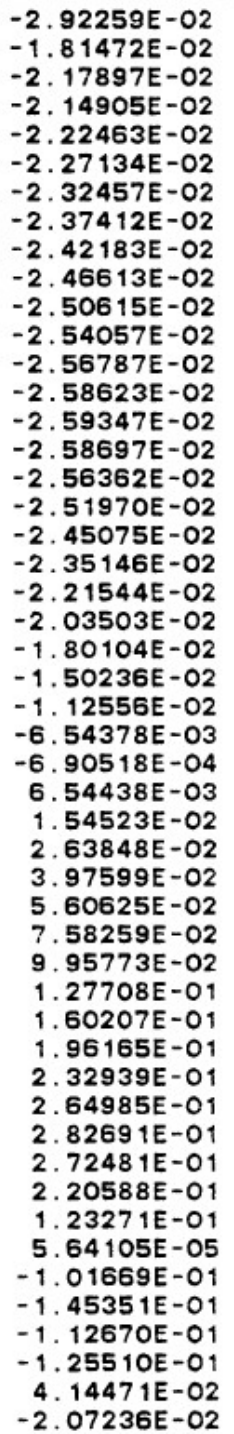 & 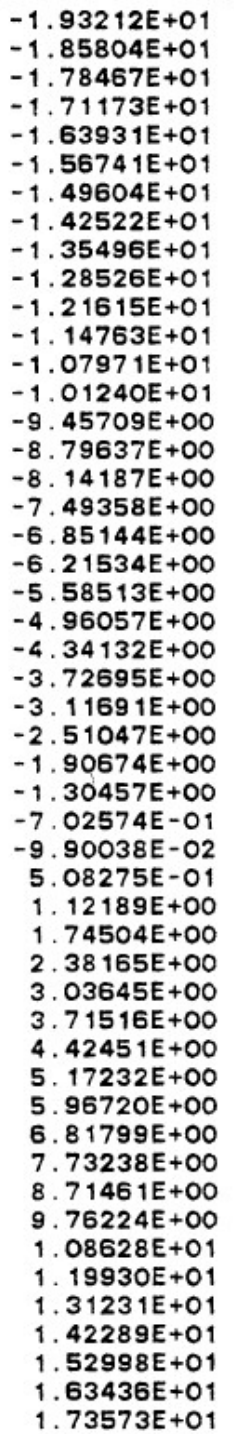 & $\begin{array}{l}-1.85804 E+01 \\
-1.78467 E+01 \\
-1.71173 E+01 \\
-1.63931 E+01 \\
-1.56741 E+01 \\
-1.49604 E+01 \\
-1.42522 E+01 \\
-1.35496 E+01 \\
-1.28526 E+01 \\
-1.21615 E+01 \\
-1.14763 E+01 \\
-1.07971 E+01 \\
-1.01240 E+01 \\
-9.45709 E+00 \\
-8.79637 E+00 \\
-8.14187 E+00 \\
-7.49358 E+00 \\
-6.85144 E+00 \\
-6.21534 E+00 \\
-5.58513 E+00 \\
-4.96057 E+00 \\
-4.34132 E+00 \\
-3.72695 E+00 \\
-3.11691 E+00 \\
-2.51047 E+00 \\
-1.90674 E+00 \\
-1.30457 E+00 \\
-7.02574 E-01 \\
-9.90038 E-02 \\
5.08275 E-01 \\
1.12189 E+00 \\
1.74504 E+00 \\
2.38165 E+00 \\
3.03645 E+00 \\
3.71516 E+00 \\
4.42451 E+00 \\
5.17232 E+00 \\
5.96720 E+00 \\
6.81799 E+00 \\
7.73238 E+00 \\
8.71461 E+00 \\
9.76224 E+00 \\
1.08628 E+01 \\
1.19930 E+01 \\
1.31231 E+01 \\
1.42289 E+01 \\
1.52998 E+01 \\
1.63436 E+01 \\
1.73573 E+01 \\
1.83810 E+01\end{array}$ \\
\hline
\end{tabular}

Figura B.3: Tabla 3 del trabajo de Paquette et al. 1986.

Coeficientes de Spline para un potencial repulsivo: $j=3$. 
TABLE 4

Spline Coefficients (Repulsive Potential): $l=1-4$

\begin{tabular}{|c|c|c|c|c|}
\hline$n$ & ${ }^{d}$ in & $d_{2 n}$ & $d_{3 n}$ & $d_{\Delta n}$ \\
\hline $\begin{array}{r}1 \\
2 \\
3 \\
4 \\
5 \\
6 \\
7 \\
8 \\
9 \\
10 \\
11 \\
12 \\
13 \\
14 \\
15 \\
16 \\
17 \\
18 \\
19 \\
20 \\
21 \\
22 \\
23 \\
24 \\
25 \\
26 \\
27 \\
28 \\
29 \\
30 \\
31 \\
32 \\
33 \\
34 \\
35 \\
36 \\
37 \\
38 \\
39 \\
40 \\
41 \\
42 \\
43 \\
44 \\
45 \\
46 \\
47 \\
48 \\
49 \\
50\end{array}$ & $\begin{array}{r}1.18229 E-02 \\
-2.36458 E-02 \\
-1.46794 \mathrm{E}-02 \\
-1.76226 \mathrm{E}-02 \\
-1.73748 \mathrm{E}-02 \\
-1.79780 \mathrm{E}-02 \\
-1.83439 \mathrm{E}-02 \\
-1.87580 \mathrm{E}-02 \\
-1.91359 \mathrm{E}-02 \\
-1.94907 \mathrm{E}-02 \\
-1.98074 \mathrm{E}-02 \\
-2.00758 \mathrm{E}-02 \\
-2.02817 \mathrm{E}-02 \\
-2.04080 \mathrm{E}-02 \\
-2.04344 \mathrm{E}-02 \\
-2.03360 \mathrm{E}-02 \\
-2.00828 \mathrm{E}-02 \\
-1.96388 \mathrm{E}-02 \\
-1.89603 \mathrm{E}-02 \\
-1.79946 \mathrm{E}-02 \\
-1.66781 \mathrm{E}-02 \\
-1.49334 \mathrm{E}-02 \\
-1.26670 \mathrm{E}-02 \\
-9.76488 \mathrm{E}-03 \\
-6.08817 \mathrm{E}-03 \\
-1.46688 \mathrm{E}-03 \\
4.30758 \mathrm{E}-03 \\
1.14919 \mathrm{E}-02 \\
2.04026 \mathrm{E}-02 \\
3.14302 \mathrm{E}-02 \\
4.50547 \mathrm{E}-02 \\
6.18616 \mathrm{E}-02 \\
8.25526 \mathrm{E}-02 \\
1.07939 \mathrm{E}-01 \\
1.38897 \mathrm{E}-01 \\
1.76234 \mathrm{E}-01 \\
2.20387 \mathrm{E}-01 \\
2.70814 \mathrm{E}-01 \\
3.24880 \mathrm{E}-01 \\
3.76096 \mathrm{E}-01 \\
4.11895 \mathrm{E}-01 \\
4.12194 \mathrm{E}-01 \\
3.52176 \mathrm{E}-01 \\
2.14860 \mathrm{E}-01 \\
1.56932 \mathrm{E}-02 \\
-1.84529 \mathrm{E}-01 \\
-3.17740 \mathrm{E}-01 \\
-3.51796 \mathrm{E}-01 \\
-2.86379 \mathrm{E}-01 \\
-2.68214 \mathrm{E}-01\end{array}$ & $\begin{array}{l}-2.36458 E-02 \\
-1.46794 E-02 \\
-1.76226 E-02 \\
-1.73748 E-02 \\
-1.79780 E-02 \\
-1.83439 E-02 \\
-1.87580 E-02 \\
-1.91359 E-02 \\
-1.94907 E-02 \\
-1.98074 E-02 \\
-2.00758 E-02 \\
-2.02817 E-02 \\
-2.04080 E-02 \\
-2.04344 E-02 \\
-2.03360 E-02 \\
-2.00828 E-02 \\
-1.96388 E-02 \\
-1.89603 E-02 \\
-1.79946 E-02 \\
-1.66781 E-02 \\
-1.49334 E-02 \\
-1.26670 E-02 \\
-9.76488 E-03 \\
-6.08817 E-03 \\
-1.46688 E-03 \\
4.30758 E-03 \\
1.14919 E-02 \\
2.04026 E-02 \\
3.14302 E-02 \\
4.50547 E-02 \\
6.18616 E-02 \\
8.25526 E-02 \\
1.07939 E-01 \\
1.38897 E-01 \\
1.76234 E-01 \\
2.20387 E-01 \\
2.70814 E-01 \\
3.24880 E-01 \\
3.76096 E-01 \\
4.11895 E-01 \\
4.12194 E-01 \\
3.52176 E-01 \\
2.14860 E-01 \\
1.56932 E-02 \\
-1.84529 E-01 \\
-3.17740 E-01 \\
-3.51796 E-01 \\
-2.86379 E-01 \\
-2.68214 E-01 \\
1.34107 E-01\end{array}$ & $\begin{array}{l}-2.55112 E+01 \\
-2.47319 E+01 \\
-2.39583 E+01 \\
-2.31882 E+01 \\
-2.24223 E+01 \\
-2.16606 E+01 \\
-2.09032 E+01 \\
-2.01502 E+01 \\
-1.94017 E+01 \\
-1.86579 E+01 \\
-1.79186 E+01 \\
-1.71842 E+01 \\
-1.64545 E+01 \\
-1.57298 E+01 \\
-1.50099 E+01 \\
-1.42949 E+01 \\
-1.35848 E+01 \\
-1.28796 E+01 \\
-1.21790 E+01 \\
-1.14830 E+01 \\
-1.07913 E+01 \\
-1.01036 E+01 \\
-9.41952 E+00 \\
-8.73845 E+00 \\
-8.05974 E+00 \\
-7.38248 E+00 \\
-6.70557 E+00 \\
-6.02763 E+00 \\
-5.34694 E+00 \\
-4.66134 E+00 \\
-3.96821 E+00 \\
-3.26426 E+00 \\
-2.54546 E+00 \\
-1.80685 E+00 \\
-1.04234 E+00 \\
-2.44487 E-01 \\
5.95659 E-01 \\
1.48870 E+00 \\
2.44673 E+00 \\
3.48274 E+00 \\
4.60901 E+00 \\
5.83413 E+00 \\
7.15818 E+00 \\
8.56675 E+00 \\
1.00269 E+01 \\
1.14908 E+01 \\
1.29104 E+01 \\
1.42538 E+01 \\
1.55127 E+01 \\
1.67029 E+01\end{array}$ & $\begin{array}{l}-2.47319 E+01 \\
-2.39583 E+01 \\
-2.31882 E+01 \\
-2.24223 E+01 \\
-2.16606 E+01 \\
-2.09032 E+01 \\
-2.01502 E+01 \\
-1.94017 E+01 \\
-1.86579 E+01 \\
-1.79186 E+01 \\
-1.71842 E+01 \\
-1.64545 E+01 \\
-1.57298 E+01 \\
-1.50099 E+01 \\
-1.42949 E+01 \\
-1.35848 E+01 \\
-1.28796 E+01 \\
-1.21790 E+01 \\
-1.14830 E+01 \\
-1.07913 E+01 \\
-1.01036 E+01 \\
-9.41952 E+00 \\
-8.73845 E+00 \\
-8.05974 E+00 \\
-7.38248 E+00 \\
-6.70557 E+00 \\
-6.02763 E+00 \\
-5.34694 E+00 \\
-4.66134 E+00 \\
-3.96821 E+00 \\
-3.26426 E+00 \\
-2.54546 E+00 \\
-1.80685 E+00 \\
-1.04234 E+00 \\
-2.44487 E-01 \\
5.95659 E-01 \\
1.48870 E+00 \\
2.44673 E+00 \\
3.48274 E+00 \\
4.60901 E+00 \\
5.83413 E+00 \\
7.15818 E+00 \\
8.56675 E+00 \\
1.00269 E+01 \\
1.14908 E+01 \\
1.29104 E+01 \\
1.42538 E+01 \\
1.55127 E+01 \\
1.67029 E+01 \\
1.78287 E+01\end{array}$ \\
\hline
\end{tabular}

Figura B.4: Tabla 4 del trabajo de Paquette et al. 1986.

Coeficientes de Spline para un potencial repulsivo: $j=4$. 
TABLE 5

SPline Coefficients (ATtractive Potential): $j=1$

\begin{tabular}{|c|c|c|c|c|}
\hline$n$ & $c_{\text {In }}^{(1)}$ & $c_{2 n}^{(1)}$ & $c_{3 n}^{(1)}$ & $c_{4 n}^{(1)}$ \\
\hline $\begin{array}{r}1 \\
2 \\
3 \\
4 \\
5 \\
6 \\
7 \\
8 \\
9 \\
10 \\
11 \\
12 \\
13 \\
14 \\
15 \\
16 \\
17 \\
18 \\
19 \\
20 \\
21 \\
22 \\
23 \\
24 \\
25 \\
26 \\
27 \\
28 \\
29 \\
30 \\
31 \\
32 \\
33 \\
34 \\
35 \\
36 \\
37 \\
38 \\
39 \\
40 \\
41 \\
42 \\
43 \\
44 \\
45 \\
46 \\
47 \\
48 \\
49 \\
50\end{array}$ & $\begin{array}{r}-4.85605 E+O O \\
9.71211 E+00 \\
-9.31384 E+00 \\
9.24600 E+00 \\
-5.09678 E+00 \\
-3.51130 E-01 \\
-5.73409 E-01 \\
1.83302 E+00 \\
-7.68878 E-01 \\
9.36371 E-01 \\
-8.65172 E-01 \\
-1.42540 E+00 \\
4.34705 E+00 \\
3.61550 E-01 \\
-3.50574 E+00 \\
2.59550 E+00 \\
-1.44563 E+00 \\
-8.64470 E-01 \\
8.82476 E-01 \\
1.35231 E-01 \\
8.48986 E-02 \\
-5.89033 E-02 \\
-1.07275 E-01 \\
-1.53774 E-01 \\
-6.39482 E-02 \\
-1.27604 E-01 \\
-3.16007 E-01 \\
-7.55843 E-02 \\
6.48945 E-02 \\
5.15827 E-02 \\
-4.79565 E-01 \\
3.59783 E-01 \\
-3.04381 E-01 \\
1.12514 E-01 \\
1.90268 E-01 \\
1.96365 E-01 \\
1.78507 E-01 \\
1.77190 E-01 \\
1.92652 E-01 \\
2.20472 E-01 \\
2.52940 E-01 \\
2.82221 E-01 \\
3.00417 E-01 \\
2.99089 E-01 \\
2.69658 E-01 \\
2.08129 E-01 \\
1.12949 E-01 \\
9.86067 E-03 \\
-1.12290 E-01 \\
-2.95924 E-02\end{array}$ & $\begin{array}{r}9.71211 E+O O \\
-9.31384 E+00 \\
9.24600 E+00 \\
-5.09678 E+00 \\
-3.51130 E-01 \\
-5.73409 E-01 \\
1.83302 E+00 \\
-7.68878 E-01 \\
9.36371 E-01 \\
-8.65172 E-01 \\
-1.42540 E+O O \\
4.34705 E+00 \\
3.61550 E-01 \\
-3.50574 E+00 \\
2.59550 E+00 \\
-1.44563 E+00 \\
-8.64470 E-01 \\
8.82476 E-01 \\
1.35231 E-01 \\
8.48986 E-02 \\
-5.89033 E-02 \\
-1.07275 E-01 \\
-1.53774 E-01 \\
-6.39482 E-02 \\
-1.27604 E-01 \\
-3.16007 E-01 \\
-7.55843 E-02 \\
6.48945 E-02 \\
5.15827 E-02 \\
-4.79565 E-01 \\
3.59783 E-01 \\
-3.04381 E-01 \\
1.12514 E-01 \\
1.90268 E-01 \\
1.96365 E-01 \\
1.78507 E-01 \\
1.77190 E-01 \\
1.92652 E-01 \\
2.20472 E-01 \\
2.52940 E-01 \\
2.82221 E-01 \\
3.00417 E-01 \\
2.99089 E-01 \\
2.69658 E-01 \\
2.08129 E-01 \\
1.12949 E-01 \\
9.86067 E-03 \\
-1.12290 E-01 \\
-2.95924 E-02 \\
1.47962 E-02\end{array}$ & $\begin{array}{l}-2.44778 E+01 \\
-2.50688 E+01 \\
-2.33288 E+01 \\
-2.38242 E+01 \\
-2.21005 E+01 \\
-2.16001 E+01 \\
-2.11839 E+01 \\
-2.09054 E+01 \\
-2.01869 E+01 \\
-1.96529 E+01 \\
-1.88943 E+01 \\
-1.83432 E+01 \\
-1.81343 E+01 \\
-1.68820 E+01 \\
-1.55430 E+01 \\
-1.50454 E+01 \\
-1.39249 E+01 \\
-1.31513 E+01 \\
-1.25851 E+01 \\
-1.18072 E+01 \\
-1.09968 E+01 \\
-1.01661 E+01 \\
-9.34947 E+00 \\
-8.55860 E+00 \\
-7.80464 E+00 \\
-7.06602 E+00 \\
-6.35803 E+00 \\
-5.72588 E+00 \\
-5.11188 E+00 \\
-4.48229 E+00 \\
-3.84033 E+00 \\
-3.31347 E+00 \\
-2.70025 E+00 \\
-2.16009 E+00 \\
-1.59292 E+00 \\
-9.80089 E-01 \\
-3.20131 E-01 \\
3.82669 E-01 \\
1.12800 E+00 \\
1.91956 E+00 \\
2.76403 E+00 \\
3.66921 E+00 \\
4.64213 E+00 \\
5.68714 E+00 \\
6.80394 E+00 \\
7.98545 E+00 \\
9.21692 E+00 \\
1.04755 E+01 \\
1.17364 E+01 \\
1.29704 E+01\end{array}$ & $\begin{array}{l}-2.50688 E+01 \\
-2.33288 E+01 \\
-2.38242 E+01 \\
-2.21005 E+01 \\
-2.16001 E+01 \\
-2.11839 E+01 \\
-2.09054 E+01 \\
-2.01869 E+01 \\
-1.96529 E+01 \\
-1.88943 E+01 \\
-1.83432 E+01 \\
-1.81343 E+01 \\
-1.68820 E+01 \\
-1.55430 E+01 \\
-1.50454 E+01 \\
-1.39249 E+01 \\
-1.31513 E+01 \\
-1.25851 E+01 \\
-1.18072 E+01 \\
-1.09968 E+01 \\
-1.01661 E+01 \\
-9.34947 E+00 \\
-8.55860 E+00 \\
-7.80464 E+00 \\
-7.06602 E+00 \\
-6.35803 E+00 \\
-5.72588 E+00 \\
-5.11188 E+00 \\
-4.48229 E+00 \\
-3.84033 E+00 \\
-3.31347 E+00 \\
-2.70025 E+00 \\
-2.16009 E+00 \\
-1.59292 E+00 \\
-9.80089 E-01 \\
-3.20131 E-01 \\
3.82669 E-01 \\
1.12800 E+00 \\
1.91956 E+00 \\
2.76403 E+00 \\
3.66921 E+00 \\
4.64213 E+00 \\
5.68714 E+00 \\
6.80394 E+00 \\
7.98545 E+00 \\
9.21692 E+00 \\
1.04755 E+01 \\
1.17364 E+01 \\
1.29704 E+01 \\
1.41973 E+01\end{array}$ \\
\hline
\end{tabular}

Figura B.5: Tabla 5 del trabajo de Paquette et al. 1986.

Coeficientes de Spline para un potencial atractivo: $j=1$. 
TABLE 6

Spline Coefficients (ATtractive Potential): $j=2$

\begin{tabular}{|c|c|c|c|c|}
\hline$n$ & $c_{1 n}^{(2)}$ & $c_{2 n}^{(2)}$ & $c_{3 n}^{(2)}$ & $c_{4 n}^{(2)}$ \\
\hline $\begin{array}{r}1 \\
2 \\
3 \\
4 \\
5 \\
6 \\
7 \\
8 \\
9 \\
10 \\
11 \\
12 \\
13 \\
14 \\
15 \\
16 \\
17 \\
18 \\
19 \\
20 \\
21 \\
22 \\
23 \\
24 \\
25 \\
26 \\
27 \\
28 \\
29 \\
30 \\
31 \\
32 \\
33 \\
34 \\
35 \\
36 \\
37 \\
38 \\
39 \\
40 \\
41 \\
42 \\
43 \\
44 \\
45 \\
46 \\
47 \\
48 \\
49 \\
50\end{array}$ & $\begin{array}{r}-3.80453 E+00 \\
7.60906 E+00 \\
-1.00677 E+O 1 \\
1.09298 E+01 \\
-3.76938 E+00 \\
-2.50868 E+00 \\
2.83415 E+00 \\
-1.06745 E+00 \\
-2.73597 E-01 \\
5.70462 E-01 \\
1.17072 E-01 \\
-4.26755 E-01 \\
1.84166 E+00 \\
-4.51420 E-02 \\
-1.68106 E+00 \\
7.40637 E-01 \\
-4.15277 E-01 \\
-5.31841 E-01 \\
7.54067 E-02 \\
3.64437 E-02 \\
8.52374 E-03 \\
-5.24956 E-02 \\
-8.76527 E-02 \\
-1.07693 E-01 \\
-1.02441 E-01 \\
-1.04497 E-01 \\
-1.16430 E-01 \\
-8.20446 E-02 \\
-5.53768 E-02 \\
-4.31036 E-02 \\
-8.94580 E-02 \\
1.74820 E-02 \\
-4.17248 E-02 \\
2.16009 E-02 \\
5.02965 E-02 \\
7.34150 E-02 \\
9.68269 E-02 \\
1.25372 E-01 \\
1.59190 E-01 \\
1.96991 E-01 \\
2.35595 E-01 \\
2.69792 E-01 \\
2.92272 E-01 \\
2.95067 E-01 \\
2.70564 E-01 \\
2.20440 E-01 \\
1.38511 E-01 \\
8.80848 E-02 \\
-7.78941 E-02 \\
3.60071 E-01\end{array}$ & 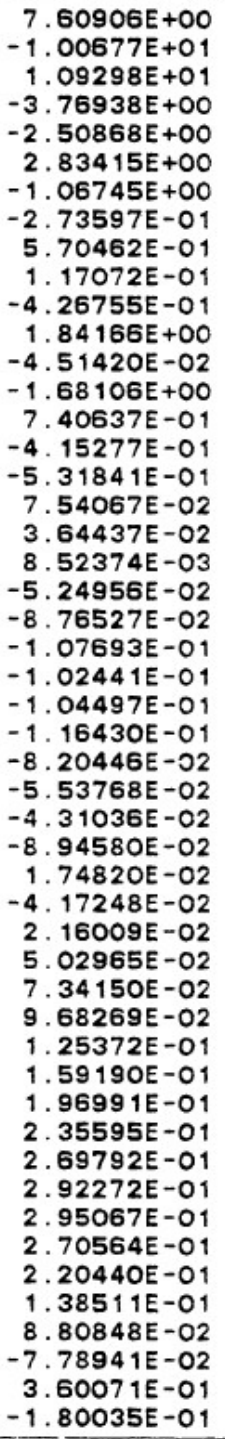 & $\begin{array}{l}-2.08526 E+01 \\
-2.11137 E+01 \\
-1.95486 E+01 \\
-2.03997 E+01 \\
-1.86277 E+01 \\
-1.77604 E+01 \\
-1.74951 E+01 \\
-1.65496 E+01 \\
-1.58604 E+01 \\
-1.52368 E+01 \\
-1.44762 E+01 \\
-1.36876 E+01 \\
-1.30014 E+01 \\
-1.18732 E+01 \\
-1.07559 E+01 \\
-1.00419 E+01 \\
-9.15028 E+00 \\
-8.35829 E+00 \\
-7.69393 E+00 \\
-7.01148 E+00 \\
-6.32029 E+00 \\
-5.62704 E+00 \\
-4.94640 E+00 \\
-4.28679 E+00 \\
-3.65303 E+00 \\
-3.04386 E+00 \\
-2.45976 E+00 \\
-1.90361 E+00 \\
-1.36715 E+00 \\
-8.43977 E-01 \\
-3.31151 E-01 \\
1.60205 E-01 \\
6.55758 E-01 \\
1.14130 E+00 \\
1.63202 E+00 \\
2.13481 E+00 \\
2.65522 E+00 \\
3.19888 E+00 \\
3.77262 E+00 \\
4.38456 E+00 \\
5.04379 E+00 \\
5.75956 E+00 \\
6.54007 E+00 \\
7.39073 E+00 \\
8.31221 E+00 \\
9.29863 E+00 \\
1.03379 E+01 \\
1.14105 E+01 \\
1.25042 E+01 \\
1.35792 E+01\end{array}$ & $\begin{array}{l}-2.11137 E+01 \\
-1.95486 E+01 \\
-2.03997 E+01 \\
-1.86277 E+01 \\
-1.77604 E+01 \\
-1.74951 E+01 \\
-1.65496 E+01 \\
-1.58604 E+01 \\
-1.52368 E+01 \\
-1.44762 E+01 \\
-1.36876 E+01 \\
-1.30014 E+01 \\
-1.18732 E+01 \\
-1.07559 E+01 \\
-1.00419 E+01 \\
-9.15028 E+00 \\
-8.35829 E+00 \\
-7.69393 E+00 \\
-7.01148 E+00 \\
-6.32029 E+00 \\
-5.62704 E+00 \\
-4.94640 E+00 \\
-4.28679 E+00 \\
-3.65303 E+00 \\
-3.04386 E+00 \\
-2.45976 E+00 \\
-1.90361 E+00 \\
-1.36715 E+00 \\
-8.43977 E-01 \\
-3.31151 E-01 \\
1.60205 E-01 \\
6.55758 E-01 \\
1.14130 E+00 \\
1.63202 E+00 \\
2.13481 E+00 \\
2.65522 E+00 \\
3.19888 E+00 \\
3.77262 E+00 \\
4.38456 E+00 \\
5.04379 E+00 \\
5.75956 E+00 \\
6.54007 E+00 \\
7.39073 E+00 \\
8.31221 E+00 \\
9.29863 E+00 \\
1.03379 E+01 \\
1.14105 E+01 \\
1.25042 E+01 \\
1.35792 E+01 \\
1.47406 E+01\end{array}$ \\
\hline
\end{tabular}

Figura B.6: Tabla 6 del trabajo de Paquette et al. 1986.

Coeficientes de Spline para un potencial atractivo: $j=2$. 
TABLE 7

Spline Coefficients (AtTractive Potential): $j=3$

\begin{tabular}{|c|c|c|c|c|}
\hline $\mathrm{n}$ & $c{ }_{1 n}^{(3)}$ & $c_{2 n}^{(3)}$ & $c_{3 n}^{(3)}$ & $c_{4 n}^{(3)}$ \\
\hline $\begin{array}{r}1 \\
2 \\
3 \\
4 \\
5 \\
6 \\
7 \\
8 \\
9 \\
10 \\
11 \\
12 \\
13 \\
14 \\
15 \\
16 \\
17 \\
18 \\
19 \\
20 \\
21 \\
22 \\
23 \\
24 \\
25 \\
26 \\
27 \\
28 \\
29 \\
30 \\
31 \\
32 \\
33 \\
34 \\
35 \\
36 \\
37 \\
38 \\
39 \\
40 \\
41 \\
42 \\
43 \\
44 \\
45 \\
46 \\
47 \\
48 \\
49 \\
50\end{array}$ & $\begin{array}{r}-2.06818 E+00 \\
4.13636 E+00 \\
-8.93460 E+00 \\
1.08282 E+01 \\
-2.97377 E+00 \\
-2.25377 E+00 \\
2.79041 E+00 \\
-1.37253 E+00 \\
-3.87183 E-01 \\
3.36588 E-01 \\
2.05509 E-01 \\
-9.13383 E-02 \\
5.02385 E-01 \\
-1.25938 E-01 \\
-6.87925 E-01 \\
8.12958 E-02 \\
-2.46616 E-01 \\
-3.03295 E-01 \\
-9.83392 E-02 \\
-7.94911 E-02 \\
-7.66879 E-02 \\
-8.63431 E-02 \\
-9.05049 E-02 \\
-9.00801 E-02 \\
-8.47287 E-02 \\
-7.78775 E-02 \\
-7.05918 E-02 \\
-5.90727 E-02 \\
-4.74236 E-02 \\
-3.62205 E-02 \\
-2.90446 E-02 \\
-8.69943 E-03 \\
-2.75832 E-04 \\
1.96726 E-02 \\
3.91533 E-02 \\
6.09268 E-02 \\
8.60213 E-02 \\
1.15234 E-01 \\
1.48588 E-01 \\
1.85161 E-01 \\
2.22451 E-01 \\
2.55866 E-01 \\
2.78501 E-01 \\
2.83080 E-01 \\
2.62093 E-01 \\
2.21026 E-01 \\
1.44689 E-01 \\
1.33750 E-01 \\
-1.00279 E-01 \\
7.07988 E-01\end{array}$ & $\begin{array}{r}4.13636 E+00 \\
-8.93460 E+00 \\
1.08282 E+01 \\
-2.97377 E+00 \\
-2.25377 E+00 \\
2.79041 E+00 \\
-1.37253 E+00 \\
-3.87183 E-01 \\
3.36588 E-01 \\
2.05509 E-01 \\
-9.13383 E-02 \\
5.02385 E-01 \\
-1.25938 E-01 \\
-6.87925 E-01 \\
8.12958 E-02 \\
-2.46616 E-01 \\
-3.03295 E-01 \\
-9.83392 E-02 \\
-7.94911 E-02 \\
-7.66879 E-02 \\
-8.63431 E-02 \\
-9.05049 E-02 \\
-9.00801 E-02 \\
-8.47287 E-02 \\
-7.78775 E-02 \\
-7.05918 E-02 \\
-5.90727 E-02 \\
-4.74236 E-02 \\
-3.62205 E-02 \\
-2.90446 E-02 \\
-8.69943 E-03 \\
-2.75832 E-04 \\
1.96726 E-02 \\
3.91533 E-02 \\
6.09268 E-02 \\
8.60213 E-02 \\
1.15234 E-01 \\
1.48588 E-01 \\
1.85161 E-01 \\
2.22451 E-01 \\
2.55866 E-01 \\
2.78501 E-01 \\
2.83080 E-01 \\
2.62093 E-01 \\
2.21026 E-01 \\
1.44689 E-01 \\
1.33750 E-01 \\
-1.00279 E-01 \\
7.07988 E-01 \\
-3.53994 E-01\end{array}$ & $\begin{array}{l}-1.54059 E+01 \\
-1.51150 E+01 \\
-1.38314 E+01 \\
-1.46921 E+01 \\
-1.29540 E+01 \\
-1.19296 E+01 \\
-1.14462 E+01 \\
-1.02930 E+01 \\
-9.46928 E+00 \\
-8.73845 E+00 \\
-7.92684 E+00 \\
-7.06591 E+00 \\
-6.22691 E+00 \\
-5.26733 E+00 \\
-4.33797 E+00 \\
-3.57372 E+00 \\
-2.78995 E+00 \\
-2.06538 E+00 \\
-1.41359 E+00 \\
-7.85408 E-01 \\
-1.76302 E-01 \\
4.14399 E-01 \\
9.84378 E-01 \\
1.53264 E+00 \\
2.05927 E+00 \\
2.56558 E+00 \\
3.05319 E+00 \\
3.52386 E+00 \\
3.98035 E+00 \\
4.42546 E+00 \\
4.86188 E+00 \\
5.29133 E+00 \\
5.71869 E+00 \\
6.14599 E+00 \\
6.57800 E+00 \\
7.01941 E+00 \\
7.47545 E+00 \\
7.95213 E+00 \\
8.45646 E+00 \\
8.99646 E+00 \\
9.58090 E+00 \\
1.02187 E+01 \\
1.09180 E+01 \\
1.16840 E+01 \\
1.25180 E+01 \\
1.34149 E+01 \\
1.53649 E+01 \\
1.63664 E+01 \\
1.73591 E+01\end{array}$ & 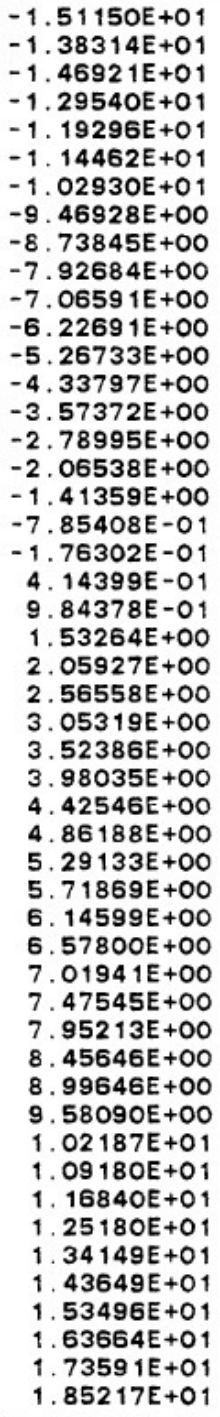 \\
\hline
\end{tabular}

Figura B.7: Tabla 7 del trabajo de Paquette et al. 1986. Coeficientes de Spline para un potencial atractivo: $j=3$. 
TABLE 8

Spline Coefficients (AtTractive Potential): $l=1-4$

\begin{tabular}{|c|c|c|c|c|}
\hline$n$ & $d_{\text {In }}$ & $d_{2 n}$ & $d_{3 n}$ & $d_{4 n}$ \\
\hline $\begin{array}{r}1 \\
2 \\
3 \\
4 \\
5 \\
6 \\
7 \\
8 \\
9 \\
10 \\
11 \\
12 \\
13 \\
14 \\
15 \\
16 \\
17 \\
18 \\
19 \\
20 \\
21 \\
22 \\
23 \\
24 \\
25 \\
26 \\
27 \\
28 \\
29 \\
30 \\
31 \\
32 \\
33 \\
34 \\
35 \\
36 \\
37 \\
38 \\
39 \\
40 \\
41 \\
42 \\
43 \\
44 \\
45 \\
46 \\
47 \\
48 \\
49 \\
50\end{array}$ & 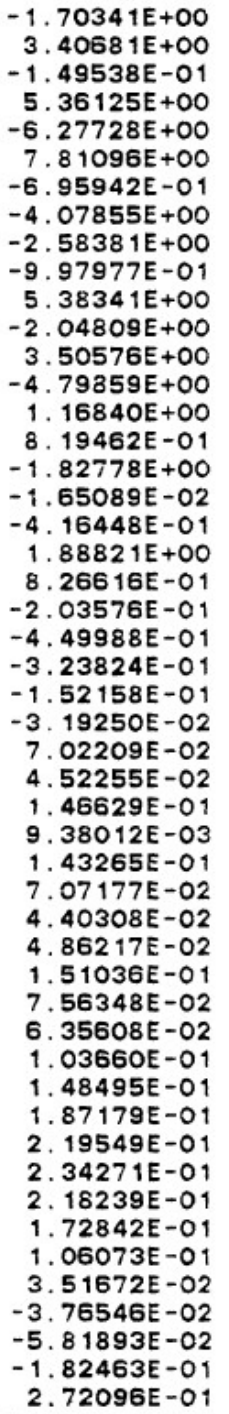 & 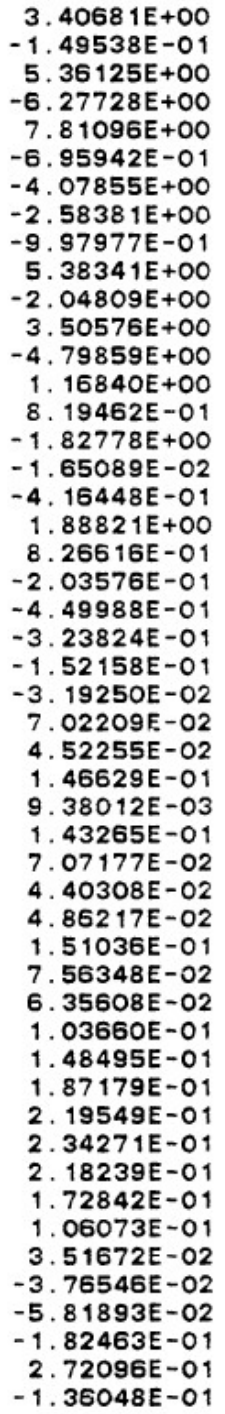 & $\begin{array}{l}-2.30677 E+01 \\
-2.36179 E+01 \\
-2.33505 E+01 \\
-2.31190 E+01 \\
-2.16008 E+01 \\
-2.15892 E+01 \\
-1.97029 E+01 \\
-1.79836 E+01 \\
-1.72432 E+01 \\
-1.71229 E+01 \\
-1.72422 E+01 \\
-1.60694 E+01 \\
-1.53881 E+01 \\
-1.38655 E+01 \\
-1.34945 E+01 \\
-1.28431 E+01 \\
-1.19950 E+01 \\
-1.15856 E+01 \\
-1.11802 E+01 \\
-1.08747 E+01 \\
-1.01161 E+01 \\
-9.15902 E+00 \\
-8.25083 E+00 \\
-7.45064 E+00 \\
-6.72817 E+00 \\
-6.04221 E+00 \\
-5.36392 E+00 \\
-4.66877 E+00 \\
-3.96278 E+00 \\
-3.22158 E+00 \\
-2.47814 E+00 \\
-1.70032 E+00 \\
-9.05521 E-01 \\
-1.00156 E-01 \\
7.16878 E-01 \\
1.57016 E+00 \\
2.44160 E+00 \\
3.32829 E+00 \\
4.23985 E+00 \\
5.18706 E+00 \\
6.17919 E+00 \\
7.22401 E+00 \\
8.32506 E+00 \\
9.47848 E+00 \\
1.06734 E+01 \\
1.18938 E+01 \\
1.31226 E+01 \\
1.43423 E+01 \\
1.55481 E+01 \\
1.67101 E+01\end{array}$ & $\begin{array}{l}-2.36179 E+01 \\
-2.33505 E+01 \\
-2.31190 E+01 \\
-2.16008 E+01 \\
-2.15892 E+01 \\
-1.97029 E+01 \\
-1.79836 E+01 \\
-1.72432 E+01 \\
-1.71229 E+01 \\
-1.72422 E+01 \\
-1.60694 E+01 \\
-1.53881 E+01 \\
-1.38655 E+01 \\
-1.34945 E+01 \\
-1.28431 E+01 \\
-1.19950 E+01 \\
-1.15856 E+01 \\
-1.11802 E+01 \\
-1.08747 E+01 \\
-1.01161 E+01 \\
-9.15902 E+00 \\
-8.25083 E+00 \\
-7.45064 E+00 \\
-6.72817 E+00 \\
-6.04221 E+00 \\
-5.36392 E+00 \\
-4.66877 E+00 \\
-3.96278 E+00 \\
-3.22158 E+00 \\
-2.47814 E+00 \\
-1.70032 E+00 \\
-9.05521 E-01 \\
-1.00156 E-01 \\
7.16878 E-01 \\
1.57016 E+00 \\
2.44160 E+00 \\
3.32829 E+00 \\
4.23985 E+00 \\
5.18706 E+00 \\
6.17919 E+00 \\
7.22401 E+00 \\
8.32506 E+00 \\
9.47848 E+00 \\
1.06734 E+01 \\
1.18938 E+01 \\
1.31226 E+01 \\
1.43423 E+01 \\
1.55481 E+01 \\
1.67101 E+01 \\
1.79374 E+01\end{array}$ \\
\hline
\end{tabular}

Figura B.8: Tabla 8 del trabajo de Paquette et al. 1986. Coeficientes de Spline para un potencial atractivo: $j=4$. 


\section{Apéndice $\mathrm{C}$}

\section{Anexo III. Matrices y vectores de las ecuaciones de difusión}

Primero, tomamos $\frac{d}{d r}\left(\ln n_{t}\right)=0$, entonces, a partir de las ecuaciones 8.12, 8.13 y 8.14, escribimos $w_{s}=w_{s}^{g}, r_{s}=r_{s}^{g}$ y $E=E^{g}$.

En las fórmulas siguientes, usaremos que $N-1=N_{\text {ion, }, 0}$, y el subíndice $e$ representa a los electrones, es decir que, e y $N$ son equivalentes. Entonces, podemos escribir a la matriz de coeficientes como sigue:

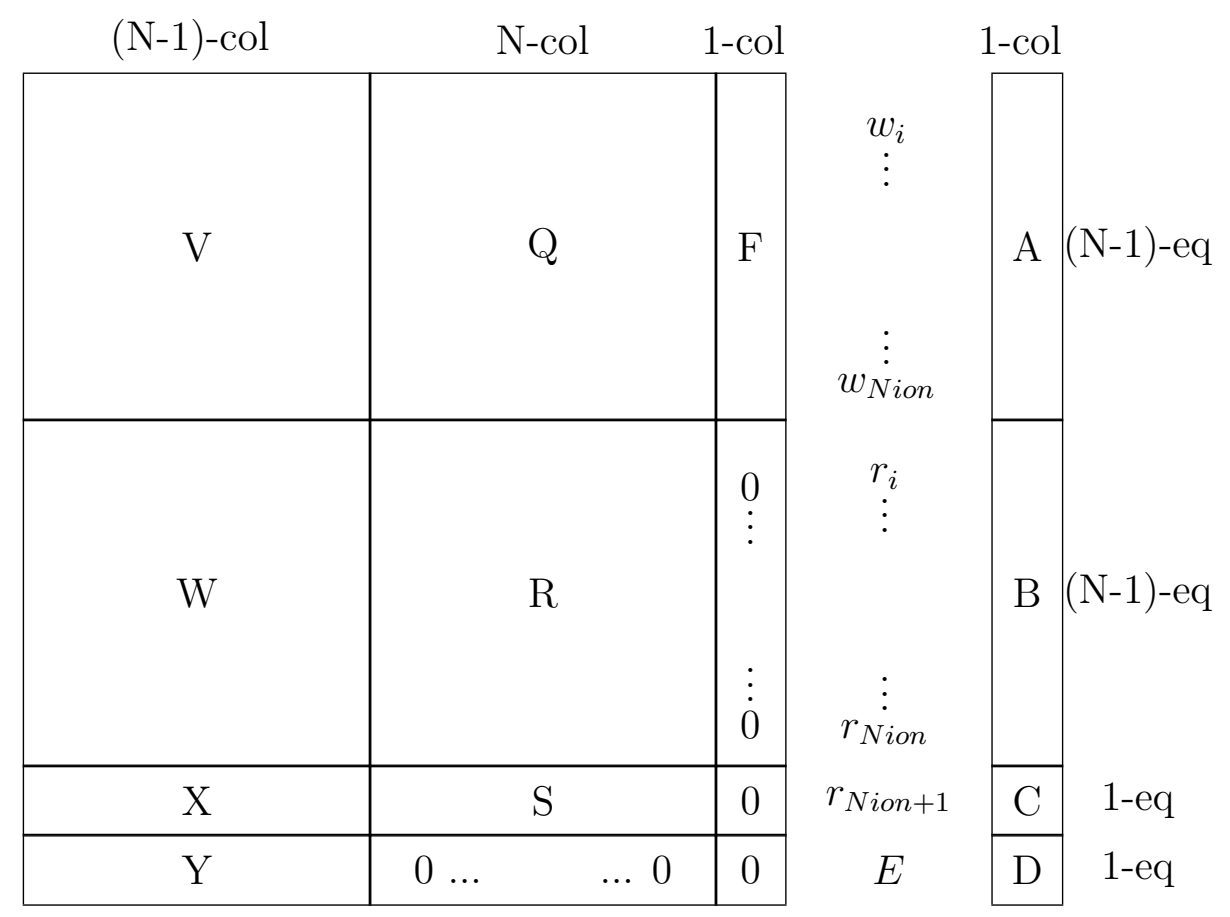

Entonces, los elementos de cada submatriz y subvector estarán definidos por las siguientes expresiones. 
$\underline{\text { Submatrix } V \text { : }}$

$$
V_{s s}=\frac{1}{e n_{s}} \sum_{t \neq s, t=1}^{N-1} K_{s t}+\frac{K_{s e}}{e n_{s}}-\frac{K_{s e} Z_{s}}{e n_{e}}
$$

para $s=1, \ldots, N-1, \mathrm{y}$,

$$
V_{s t}=-\frac{1}{e n_{s}} K_{s t}-\frac{K_{s e} Z_{t}}{e n_{e}} \frac{n_{t}}{n_{s}}
$$

para $t \neq s$, y $s, t=1, \ldots, N-1$.

Submatrix Q:

$$
Q_{s, N-1+s}=-\frac{1}{e n_{s}} \sum_{t \neq s, t=1}^{N-1} K_{s t} Z_{s t} \frac{m_{t}}{m_{s t}}
$$

para $s=1, \ldots, N-1, \mathrm{y}$,

$$
Q_{s, N-1+t}=\frac{1}{e n_{s}} K_{s t} Z_{s t} \frac{m_{s}}{m_{s t}}
$$

para $t \neq s, s=1, \ldots, N-1$ y $t=1, \ldots, N$.

Submatrix F:

$$
F_{s, 2 N}=-Z_{s}
$$

para $s=1, \ldots, N-1$.

$\underline{\text { Submatrix } W}$ :

$$
W_{N-1+s, s}=-\frac{5}{2} \frac{1}{n_{s}} \sum_{t \neq s, t=1}^{N-1} K_{s t} Z_{s t} \frac{m_{t}}{m_{s t}}-\frac{5}{2} \frac{1}{n_{s}} K_{s e} Z_{s e} \frac{m_{e}}{m_{s e}}
$$

para $s=1, \ldots, N-1$, con $m_{s e}=m_{s}+m_{e}$,

$$
W_{N-1+s, t}=\frac{5}{2} \frac{1}{n_{s}} K_{s t} Z_{s t} \frac{m_{t}}{m_{s t}}+\frac{5}{2} \frac{1}{n_{s}} K_{s e} Z_{s e} \frac{m_{e}}{m_{s e}} \frac{1}{n_{e}} Z_{t} n_{t}
$$

para $t \neq s$, y $s, t=1, \ldots, N-1$. 
Submatrix R:

$$
R_{N-1+s, N-1+s}=\frac{2}{5} \frac{1}{n_{s}} K_{s s} Z_{s s}^{\prime \prime}+\frac{1}{n_{s}} \sum_{t \neq s, t=1}^{N} K_{s t}\left[3 \frac{m_{s}^{2}}{m_{s t}^{2}}+\frac{m_{t}^{2}}{m_{s t}^{2}} Z_{s t}^{\prime}+\frac{4}{5} \frac{m_{s} m_{t}}{m_{s t}^{2}} Z_{s t}^{\prime \prime}\right]
$$

para $s=1, \ldots, N-1$.

$$
R_{N-1+s, N-1+t}=-\frac{1}{n_{s}} K_{s t} \frac{m_{s} m_{t}}{m_{s t}^{2}}\left[3+Z_{s t}^{\prime}-\frac{4}{5} Z_{s t}^{\prime \prime}\right]
$$

para $t \neq s$, y $s=1, \ldots, N-1$, y $t=1, \ldots, N$

$\underline{\text { Submatrix } X}$ :

$$
X_{2 N-1, t}=-\frac{5}{2} \frac{1}{n_{e}}\left\{Z_{t} \frac{n_{t}}{n_{e}}\left[\sum_{u=1}^{N-1} K_{e u} Z_{e u} \frac{m_{u}}{m_{u e}}\right]-K_{e t} Z_{e t} \frac{m_{t}}{m_{e t}}\right\}
$$

para $t=1, \ldots, N-1$.

\section{$\underline{\text { Submatrix } S}$ :}

$$
S_{N, t}=-\frac{1}{n_{e}} K_{e t} \frac{m_{e} m_{t}}{m_{e t}^{2}}\left[3+Z_{e t}^{\prime}-\frac{4}{5} Z_{e t}^{\prime \prime}\right]
$$

para $t=1, \ldots, N-1$.

$$
S_{N, N}=\frac{2}{5} \frac{1}{n_{e}} K_{e e} Z_{e e}^{\prime \prime}+\frac{1}{n_{e}} \sum_{t=1}^{N-1} K_{e t}\left[3 \frac{m_{e}^{2}}{m_{e t}^{2}}+\frac{m_{t}^{2}}{m_{e t}^{2}} Z_{e t}^{\prime}+\frac{4}{5} \frac{m_{e} m_{t}}{m_{e t}^{2}} Z_{e t}^{\prime \prime}\right]
$$

para $t=1, \ldots, N-1$.

Submatrix $Y$ :

$$
Y_{2 N, t}=\left(\mu_{t}+\mu_{e} Z_{t}\right) n_{t}
$$

para $t=1, \ldots, N-1$.

Submatrix A:

$$
A_{s}=-\mu_{s} \frac{M_{u} g_{s}}{e}-\frac{k_{B} T}{e} \frac{d}{d r}(\ln T)
$$

para $s=1, \ldots, N-1$. 
Submatrix B:

$$
B_{N-1+s}=-\frac{5}{2} k_{B} T \frac{d}{d r}(\ln T)
$$

para $s=1, \ldots, N-1$.

$\underline{\text { Submatrix } C}$ :

$$
C_{2 N-1}=-\frac{5}{2} k_{B} T \frac{d}{d r}(\ln T)
$$

Submatrix D:

$$
D_{2 N}=0
$$




\section{Apéndice D}

\section{Anexo IV. Símbolos químicos}

Aquí mostramos un listado de los elementos químicos mencionados en este trabajo y su correspondiente símbolo.
$\mathrm{Au}$, oro.
Hg, mercurio.
Pt, platino.
$\mathrm{Ba}$, bario.
Li, litio.
$\mathrm{S}$, azufre.
C, carbono.
Mg, magnesio.
Sc, escandio.
Ca, calcio.
Mn, manganeso.
$\mathrm{Si}$, silicio.
$\mathrm{Cr}$, cromo.
$\mathrm{N}$, nitrógeno.
$\mathrm{Sr}$, estroncio.
Eu, europio.
$\mathrm{Na}$, sodio.
Ti, titanio.
Fe, hierro.
Nd, neodimio.
Xe, xenón.
$\mathrm{Ga}$, galio.
$\mathrm{O}$, oxígeno.
$\mathbf{Y}$, itrio.
H, hidrógeno.
$\mathbf{P}$, fósforo.
Zn, zinc.
He, helio.
Pr, praseodimio.
$\mathrm{Zr}$, zirconio. 